\title{
Oxidative Sulfonylation of Hydrazones Enabled by Synergistic Copper/Silver Catalysis
}

\footnotetext{
Jun $\mathrm{Xu},{ }^{\dagger, \dagger}$ Chao Shen,${ }^{\S}$ Xian Qin, ${ }^{\dagger}$ Jie Wu, ${ }^{\dagger}$ Pengfei Zhang, ${ }^{*, \S}$ and Xiaogang Liu*, ${ }^{*},, \dagger$

${ }^{\dagger}$ Department of Chemistry, National University of Singapore, 3 Science Drive 3, Singapore, 117543, Singapore.

* Center for Functional Materials, National University of Singapore Suzhou Research Institute, Suzhou 215123, China.

$\S$ College of Material Chemistry and Chemical Engineering, Hangzhou Normal University, Hangzhou 311121, China.
}

Corresponding to: Email: chxyzpf@hotmail.com (Zhang, P.),chmlx@nus.edu.sg (Liu,X.)

\section{Supporting Information}

\section{Table of contents}

1. Optimization of Reaction Conditions

2. Mechanism Investigation

3. X-ray Crystal Data for $\mathbf{3 g}$ and $\mathbf{5 n}$

4. Copies of NMR and HRMS Spectra 


\section{Optimization of Reaction Conditions}

Scheme S1. Optimization of Auxiliary Groups ${ }^{a, b}$

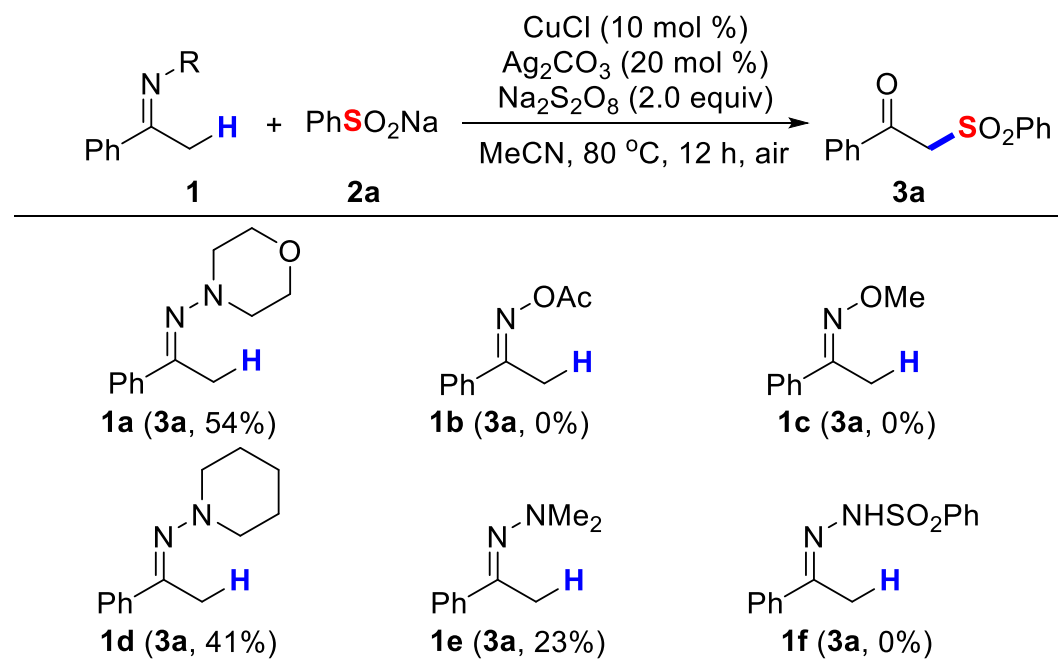

${ }^{\text {a }}$ Reaction conditions: 1 ( $\left.0.2 \mathrm{mmol}\right), \mathbf{2 a}\left(2.0\right.$ equiv), $\mathrm{CuCl}(10 \mathrm{~mol} \%), \mathrm{Ag}_{2} \mathrm{CO}_{3}(20 \mathrm{~mol} \%), \mathrm{Na}_{2} \mathrm{~S}_{2} \mathrm{O}_{8}$ (2.0 equiv), $\mathrm{MeCN}(1.0 \mathrm{~mL}), 80^{\circ} \mathrm{C}, 12 \mathrm{~h}$, air. ${ }^{\mathrm{b}}$ Isolated yields.

Table S1. Optimization of Catalysts ${ }^{a}$

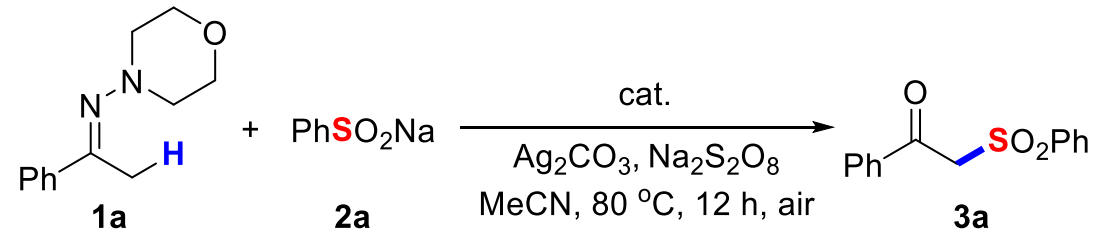

\begin{tabular}{cccccc}
\hline entry & catalyst & cocatalyst & oxidant & solvent & yield $(\%)^{b}$ \\
\hline 1 & $\mathrm{CuCl}$ & $\mathrm{Ag}_{2} \mathrm{CO}_{3}$ & $\mathrm{Na}_{2} \mathrm{~S}_{2} \mathrm{O}_{8}$ & $\mathrm{MeCN}$ & 54 \\
2 & $\mathrm{Cu}_{2} \mathrm{O}$ & $\mathrm{Ag}_{2} \mathrm{CO}_{3}$ & $\mathrm{Na}_{2} \mathrm{~S}_{2} \mathrm{O}_{8}$ & $\mathrm{MeCN}$ & 65 \\
3 & $\mathrm{CuI}$ & $\mathrm{Ag}_{2} \mathrm{CO}_{3}$ & $\mathrm{Na}_{2} \mathrm{~S}_{2} \mathrm{O}_{8}$ & $\mathrm{MeCN}$ & 59 \\
4 & $\mathrm{CuBr}$ & $\mathrm{Ag}_{2} \mathrm{CO}_{3}$ & $\mathrm{Na}_{2} \mathrm{~S}_{2} \mathrm{O}_{8}$ & $\mathrm{MeCN}$ & 50 \\
5 & $\mathrm{Cu}(\mathrm{OAc})_{2}$ & $\mathrm{Ag}_{2} \mathrm{CO}_{3}$ & $\mathrm{Na}_{2} \mathrm{~S}_{2} \mathrm{O}_{8}$ & $\mathrm{MeCN}$ & trace \\
6 & $\mathrm{CuCl}$ & $\mathrm{Ag}_{2} \mathrm{CO}_{3}$ & $\mathrm{Na}_{2} \mathrm{~S}_{2} \mathrm{O}_{8}$ & $\mathrm{MeCN}$ & trace \\
7 & $\mathrm{CuO}$ & $\mathrm{Ag}_{2} \mathrm{CO}_{3}$ & $\mathrm{Na}_{2} \mathrm{~S}_{2} \mathrm{O}_{8}$ & $\mathrm{MeCN}$ & trace \\
8 & $\mathrm{FeCl}_{2}$ & $\mathrm{Ag}_{2} \mathrm{CO}_{3}$ & $\mathrm{Na}_{2} \mathrm{~S}_{2} \mathrm{O}_{8}$ & $\mathrm{MeCN}$ & 0 \\
9 & $\mathrm{CoCl}_{2}$ & $\mathrm{Ag}_{2} \mathrm{CO}_{3}$ & $\mathrm{Na}_{2} \mathrm{~S}_{2} \mathrm{O}_{8}$ & $\mathrm{MeCN}$ & 0 \\
10 & $\mathrm{NiCl}_{2}$ & $\mathrm{Ag}_{2} \mathrm{CO}_{3}$ & $\mathrm{Na}_{2} \mathrm{~S}_{2} \mathrm{O}_{8}$ & $\mathrm{MeCN}$ & 0 \\
11 & - & $\mathrm{Ag}_{2} \mathrm{CO}_{3}$ & $\mathrm{Na}_{2} \mathrm{~S}_{2} \mathrm{O}_{8}$ & $\mathrm{MeCN}$ & 0 \\
\hline
\end{tabular}

${ }^{a}$ Reaction conditions: 1a $(0.2 \mathrm{mmol}), \mathbf{2 a}\left(2.0\right.$ equiv), catalyst $(10 \mathrm{~mol} \%), \mathrm{Ag}_{2} \mathrm{CO}_{3}(20 \mathrm{~mol} \%)$, 
$\mathrm{Na}_{2} \mathrm{~S}_{2} \mathrm{O}_{8}$ (2.0 equiv), $\mathrm{MeCN}(1.0 \mathrm{~mL}), 80{ }^{\circ} \mathrm{C}, 12 \mathrm{~h}$, air. ${ }^{\mathrm{b}}$ Isolated yields.

Table S2. Optimization of Cocatalysts ${ }^{a}$

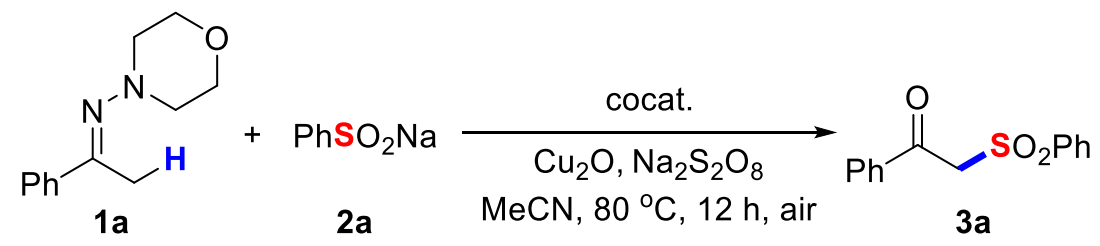

\begin{tabular}{cccccc}
\hline entry & catalyst & cocatalyst & oxidant & solvent & yield $(\%)^{b}$ \\
\hline 1 & $\mathrm{Cu}_{2} \mathrm{O}$ & $\mathrm{Ag}_{2} \mathrm{CO}_{3}$ & $\mathrm{Na}_{2} \mathrm{~S}_{2} \mathrm{O}_{8}$ & $\mathrm{MeCN}$ & 65 \\
2 & $\mathrm{Cu}_{2} \mathrm{O}$ & $\mathrm{AgSbF}_{6}$ & $\mathrm{Na}_{2} \mathrm{~S}_{2} \mathrm{O}_{8}$ & $\mathrm{MeCN}$ & 48 \\
3 & $\mathrm{Cu}_{2} \mathrm{O}$ & $\mathrm{Ag}_{2} \mathrm{O}$ & $\mathrm{Na}_{2} \mathrm{~S}_{2} \mathrm{O}_{8}$ & $\mathrm{MeCN}$ & 35 \\
4 & $\mathrm{Cu}_{2} \mathrm{O}$ & - & $\mathrm{Na}_{2} \mathrm{~S}_{2} \mathrm{O}_{8}$ & $\mathrm{MeCN}$ & 15 \\
\hline
\end{tabular}

${ }^{a}$ Reaction conditions: 1a $(0.2 \mathrm{mmol}), \mathbf{2 a}\left(2.0\right.$ equiv), $\mathrm{Cu}_{2} \mathrm{O}(10 \mathrm{~mol} \%)$, cocatalyst $(20 \mathrm{~mol} \%)$, $\mathrm{Na}_{2} \mathrm{~S}_{2} \mathrm{O}_{8}$ (2.0 equiv), $\mathrm{MeCN}(1.0 \mathrm{~mL}), 80{ }^{\circ} \mathrm{C}, 12 \mathrm{~h}$, air. ${ }^{\mathrm{b}}$ Isolated yields.

Table S3. Optimization of Oxidants ${ }^{a}$

\begin{tabular}{|c|c|c|c|c|c|}
\hline & $\overbrace{1 a}^{H}$ & $\begin{array}{l}\mathrm{hSO}_{2} \mathrm{Na} \\
2 \mathrm{a}\end{array}$ & $\begin{array}{c}\text { oxidant } \\
\mathrm{Cu} \mathrm{Cu}_{2} \mathrm{O}, \mathrm{Ag}_{2} \mathrm{CO}_{3} \\
\mathrm{MeCN}, 80^{\circ} \mathrm{C}, 12 \mathrm{~h} \text {, air }\end{array}$ & $\stackrel{O}{\mu}$ & \\
\hline entry & catalyst & cocatalyst & oxidant & solvent & yield $(\%)^{b}$ \\
\hline 1 & $\mathrm{Cu}_{2} \mathrm{O}$ & $\mathrm{Ag}_{2} \mathrm{CO}_{3}$ & $\mathrm{Na}_{2} \mathrm{~S}_{2} \mathrm{O}_{8}$ & $\mathrm{MeCN}$ & 65 \\
\hline 2 & $\mathrm{Cu}_{2} \mathrm{O}$ & $\mathrm{Ag}_{2} \mathrm{CO}_{3}$ & $\mathrm{~K}_{2} \mathrm{~S}_{2} \mathrm{O}_{8}$ & $\mathrm{MeCN}$ & 72 \\
\hline 3 & $\mathrm{Cu}_{2} \mathrm{O}$ & $\mathrm{Ag}_{2} \mathrm{CO}_{3}$ & DTBP & $\mathrm{MeCN}$ & trace \\
\hline 4 & $\mathrm{Cu}_{2} \mathrm{O}$ & $\mathrm{Ag}_{2} \mathrm{CO}_{3}$ & TBHP & $\mathrm{MeCN}$ & 21 \\
\hline 5 & $\mathrm{Cu}_{2} \mathrm{O}$ & $\mathrm{Ag}_{2} \mathrm{CO}_{3}$ & $\mathrm{PhI}(\mathrm{OAc})_{2}$ & $\mathrm{MeCN}$ & trace \\
\hline 6 & $\mathrm{Cu}_{2} \mathrm{O}$ & $\mathrm{Ag}_{2} \mathrm{CO}_{3}$ & - & $\mathrm{MeCN}$ & 18 \\
\hline
\end{tabular}

${ }^{\text {a }}$ Reaction conditions: 1a (0.2 mmol), 2a (2.0 equiv), $\mathrm{Cu}_{2} \mathrm{O}$ (10 mol \%), $\mathrm{Ag}_{2} \mathrm{CO}_{3}(20 \mathrm{~mol} \%)$, oxidant (2.0 equiv), $\mathrm{MeCN}(1.0 \mathrm{~mL}), 80{ }^{\circ} \mathrm{C}, 12 \mathrm{~h}$, air. ${ }^{\mathrm{b}}$ Isolated yields. 
Table S4. Optimization of Solvents ${ }^{a}$

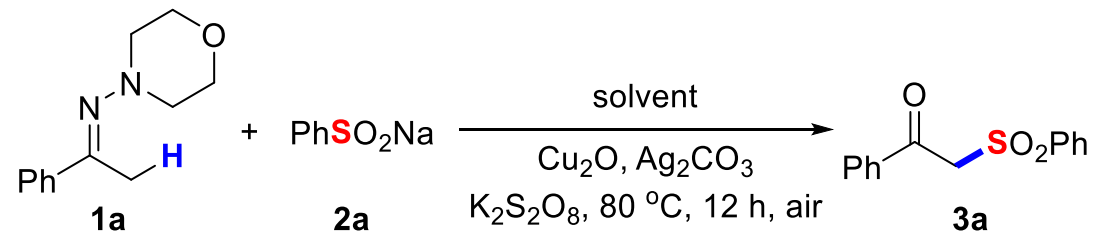

\begin{tabular}{cccccc}
\hline entry & catalyst & cocatalyst & oxidant & solvent & yield $(\%)^{b}$ \\
\hline 1 & $\mathrm{Cu}_{2} \mathrm{O}$ & $\mathrm{Ag}_{2} \mathrm{CO}_{3}$ & $\mathrm{~K}_{2} \mathrm{~S}_{2} \mathrm{O}_{8}$ & MeCN & 72 \\
2 & $\mathrm{Cu}_{2} \mathrm{O}$ & $\mathrm{Ag}_{2} \mathrm{CO}_{3}$ & $\mathrm{~K}_{2} \mathrm{~S}_{2} \mathrm{O}_{8}$ & acetone & 73 \\
3 & $\mathrm{Cu}_{2} \mathrm{O}$ & $\mathrm{Ag}_{2} \mathrm{CO}_{3}$ & $\mathrm{~K}_{2} \mathrm{~S}_{2} \mathrm{O}_{8}$ & dioxane & trace \\
4 & $\mathrm{Cu}_{2} \mathrm{O}$ & $\mathrm{Ag}_{2} \mathrm{CO}_{3}$ & $\mathrm{~K}_{2} \mathrm{~S}_{2} \mathrm{O}_{8}$ & toluene & 35 \\
5 & $\mathrm{Cu}_{2} \mathrm{O}$ & $\mathrm{Ag}_{2} \mathrm{CO}_{3}$ & $\mathrm{~K}_{2} \mathrm{~S}_{2} \mathrm{O}_{8}$ & EtOAc & trace \\
6 & $\mathrm{Cu}_{2} \mathrm{O}$ & $\mathrm{Ag}_{2} \mathrm{CO}_{3}$ & $\mathrm{~K}_{2} \mathrm{~S}_{2} \mathrm{O}_{8}$ & DMSO & 28 \\
7 & $\mathrm{Cu}_{2} \mathrm{O}$ & $\mathrm{Ag}_{2} \mathrm{CO}_{3}$ & $\mathrm{~K}_{2} \mathrm{~S}_{2} \mathrm{O}_{8}$ & DCE & 83 \\
\hline
\end{tabular}

${ }^{\text {a }}$ Reaction conditions: $1 \mathbf{a}(0.2 \mathrm{mmol}), \mathbf{2 a}$ (2.0 equiv), $\mathrm{Cu}_{2} \mathrm{O}(10 \mathrm{~mol} \%), \mathrm{Ag}_{2} \mathrm{CO}_{3}(20 \mathrm{~mol} \%), \mathrm{K}_{2} \mathrm{~S}_{2} \mathrm{O}_{8}$ (2.0 equiv), solvent $(1.0 \mathrm{~mL}), 80^{\circ} \mathrm{C}, 12 \mathrm{~h}$, air. ${ }^{\mathrm{b}}$ Isolated yields. $\mathrm{DMSO}=$ dimethyl sulfoxide; $\mathrm{DCE}=$ 1,2-dichloroethane.

Table S5. Optimization of Reaction Time ${ }^{a}$

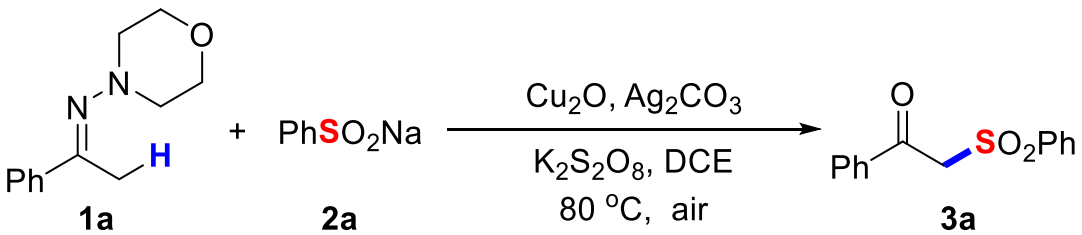

\begin{tabular}{cccccc}
\hline entry & catalyst & cocatalyst & oxidant & solvent & yield $(\%)^{b}$ \\
\hline 1 & $\mathrm{Cu}_{2} \mathrm{O}$ & $\mathrm{Ag}_{2} \mathrm{CO}_{3}$ & $\mathrm{~K}_{2} \mathrm{~S}_{2} \mathrm{O}_{8}$ & DCE & 83 \\
$2^{c}$ & $\mathrm{Cu}_{2} \mathrm{O}$ & $\mathrm{Ag}_{2} \mathrm{CO}_{3}$ & $\mathrm{~K}_{2} \mathrm{~S}_{2} \mathrm{O}_{8}$ & DCE & 85 \\
$3^{d}$ & $\mathrm{Cu}_{2} \mathrm{O}$ & $\mathrm{Ag}_{2} \mathrm{CO}_{3}$ & $\mathrm{~K}_{2} \mathrm{~S}_{2} \mathrm{O}_{8}$ & DCE & 44 \\
\hline
\end{tabular}

${ }^{\text {a }}$ Reaction conditions: 1a (0.2 mmol), $2 \mathbf{a}$ (2.0 equiv), $\mathrm{Cu}_{2} \mathrm{O}(10 \mathrm{~mol} \%), \mathrm{Ag}_{2} \mathrm{CO}_{3}(20 \mathrm{~mol} \%), \mathrm{K}_{2} \mathrm{~S}_{2} \mathrm{O}_{8}$ (2.0 equiv), DCE $(1.0 \mathrm{~mL}), 80{ }^{\circ} \mathrm{C}, 12 \mathrm{~h}$, air. ${ }^{\mathrm{b}}$ Isolated yields. ${ }^{\mathrm{c}}$ The reaction was performed for $3 \mathrm{~h}$. ${ }^{\mathrm{d}}$ The reaction was performed for $1 \mathrm{~h}$. 
Table S6. Optimization of Reaction Temperature ${ }^{a}$

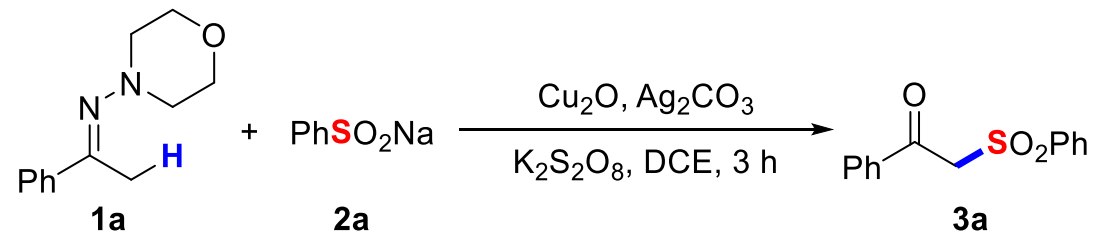

\begin{tabular}{cccccc}
\hline entry & catalyst & cocatalyst & oxidant & solvent & yield $(\%)^{b}$ \\
\hline 1 & $\mathrm{Cu}_{2} \mathrm{O}$ & $\mathrm{Ag}_{2} \mathrm{CO}_{3}$ & $\mathrm{~K}_{2} \mathrm{~S}_{2} \mathrm{O}_{8}$ & DCE & 85 \\
$2^{c}$ & $\mathrm{Cu}_{2} \mathrm{O}$ & $\mathrm{Ag}_{2} \mathrm{CO}_{3}$ & $\mathrm{~K}_{2} \mathrm{~S}_{2} \mathrm{O}_{8}$ & DCE & 46 \\
$3^{d}$ & $\mathrm{Cu}_{2} \mathrm{O}$ & $\mathrm{Ag}_{2} \mathrm{CO}_{3}$ & $\mathrm{~K}_{2} \mathrm{~S}_{2} \mathrm{O}_{8}$ & DCE & 82 \\
\hline
\end{tabular}

${ }^{\text {a }}$ Reaction conditions: $1 \mathbf{a}(0.2 \mathrm{mmol}), \mathbf{2 a}$ (2.0 equiv), $\mathrm{Cu}_{2} \mathrm{O}(10 \mathrm{~mol} \%), \mathrm{Ag}_{2} \mathrm{CO}_{3}(20 \mathrm{~mol} \%), \mathrm{K}_{2} \mathrm{~S}_{2} \mathrm{O}_{8}$ (2.0 equiv), DCE $(1.0 \mathrm{~mL}), 80{ }^{\circ} \mathrm{C}, 3 \mathrm{~h}$, air. ${ }^{\mathrm{b}}$ Isolated yields. ${ }^{\mathrm{c}}$ The reaction was performed at $50{ }^{\circ} \mathrm{C}$. ${ }^{\mathrm{d}}$ The reaction was performed at $100^{\circ} \mathrm{C}$.

Table S7. Study the Actions of Catalyst, Cocatalyst and Oxidant ${ }^{a}$

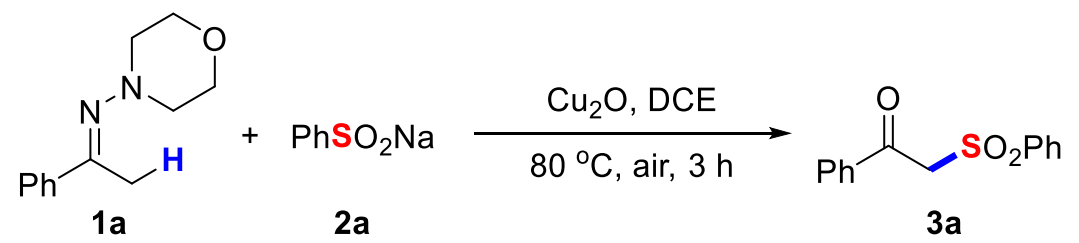

\begin{tabular}{cccccc} 
& $\mathbf{1 a}$ & $\mathbf{2 a}$ & \multicolumn{3}{c}{ 3a } \\
\hline entry & catalyst & cocatalyst & oxidant & solvent & yield $(\%)^{b}$ \\
\hline 1 & $\mathrm{Cu}_{2} \mathrm{O}$ & $\mathrm{Ag}_{2} \mathrm{CO}_{3}$ & $\mathrm{~K}_{2} \mathrm{~S}_{2} \mathrm{O}_{8}$ & DCE & 85 \\
$2^{c}$ & $\mathrm{Cu}_{2} \mathrm{O}$ & - & - & DCE & 28 \\
$3^{d}$ & $\mathrm{Cu}_{2} \mathrm{O}$ & $\mathrm{Ag}_{2} \mathrm{CO}_{3}$ & - & DCE & 53 \\
\hline
\end{tabular}

${ }^{\text {a }}$ Reaction conditions: $1 \mathbf{a}(0.2 \mathrm{mmol}), \mathbf{2 a}\left(2.0\right.$ equiv), $\mathrm{Cu}_{2} \mathrm{O}(10 \mathrm{~mol} \%), \mathrm{Ag}_{2} \mathrm{CO}_{3}(20 \mathrm{~mol} \%), \mathrm{K}_{2} \mathrm{~S}_{2} \mathrm{O}_{8}$ (2.0 equiv), DCE $(1.0 \mathrm{~mL}), 80{ }^{\circ} \mathrm{C}, 3 \mathrm{~h}$, air. ${ }^{\mathrm{b}}$ Isolated yields. ${ }^{\mathrm{c}} 1$ equivalent of $\mathrm{Cu}_{2} \mathrm{O}$ was used. d 1 equivalent of $\mathrm{Ag}_{2} \mathrm{CO}_{3}$ was used. 
Table S8. Optimization of the Level of Water Content ${ }^{a}$
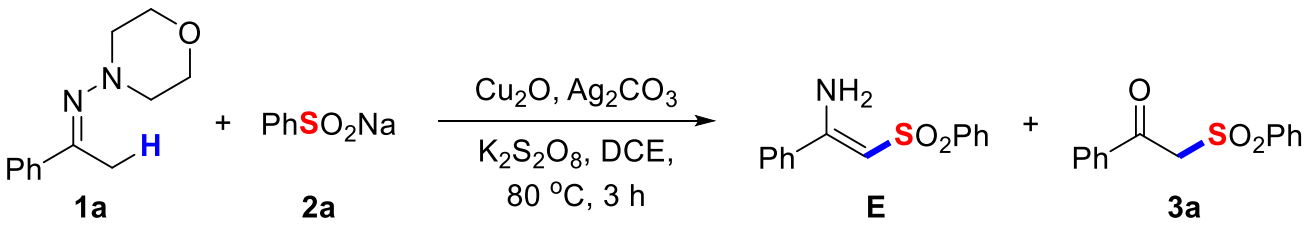

\begin{tabular}{cccc}
\hline entry & DCE (Water Content) & yield of $\mathbf{E}(\%)^{b}$ & yield of $\mathbf{3 a}(\%)^{b}$ \\
\hline 1 & $30 \mathrm{ppm}$ & 82 & trace \\
2 & $1000 \mathrm{ppm}$ & 78 & trace \\
3 & $2000 \mathrm{ppm}$ & 66 & 23 \\
4 & $3000 \mathrm{ppm}$ & 32 & 60 \\
5 & $5000 \mathrm{ppm}$ & 13 & 76 \\
6 & $7000 \mathrm{ppm}$ & trace & 84 \\
\hline
\end{tabular}

${ }^{\mathrm{a}}$ Reaction conditions: 1a $(0.3 \mathrm{mmol}), \mathbf{2 a}$ (2.0 equiv), $\mathrm{Cu}_{2} \mathrm{O}(10 \mathrm{~mol} \%), \mathrm{Ag}_{2} \mathrm{CO}_{3}(20 \mathrm{~mol} \%), \mathrm{K}_{2} \mathrm{~S}_{2} \mathrm{O}_{8}$ (2.0 equiv), DCE $(1.5 \mathrm{~mL}), 80{ }^{\circ} \mathrm{C}, 3 \mathrm{~h} .{ }^{\mathrm{b}}$ Isolated yields.

Table S9. Optimization of the Dosage of $\mathrm{PhSO}_{2} \mathrm{Na}$ and Catalyst ${ }^{a}$

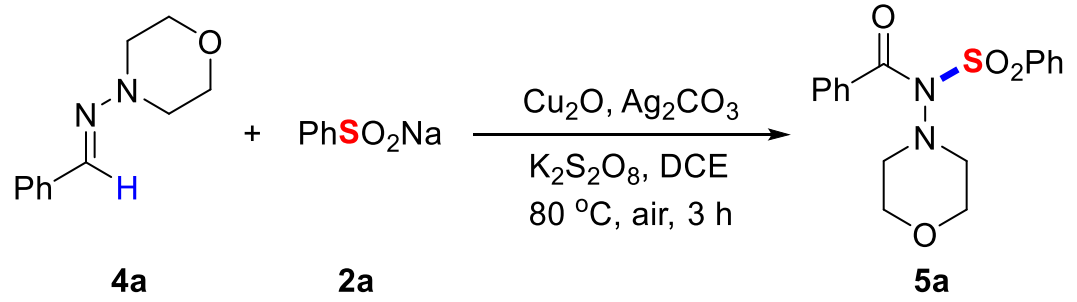

\begin{tabular}{cccccc}
\hline entry & catalyst & cocatalyst & oxidant & solvent & yield $(\%)^{b}$ \\
\hline 1 & $\mathrm{Cu}_{2} \mathrm{O}$ & $\mathrm{Ag}_{2} \mathrm{CO}_{3}$ & $\mathrm{~K}_{2} \mathrm{~S}_{2} \mathrm{O}_{8}$ & DCE & 75 \\
$2^{c}$ & $\mathrm{Cu}_{2} \mathrm{O}$ & $\mathrm{Ag}_{2} \mathrm{CO}_{3}$ & $\mathrm{~K}_{2} \mathrm{~S}_{2} \mathrm{O}_{8}$ & DCE & 40 \\
$3^{d}$ & $\mathrm{Cu}_{2} \mathrm{O}$ & $\mathrm{Ag}_{2} \mathrm{CO}_{3}$ & $\mathrm{~K}_{2} \mathrm{~S}_{2} \mathrm{O}_{8}$ & DCE & 68 \\
$4^{e}$ & $\mathrm{Cu}_{2} \mathrm{O}$ & $\mathrm{Ag}_{2} \mathrm{CO}_{3}$ & $\mathrm{~K}_{2} \mathrm{~S}_{2} \mathrm{O}_{8}$ & DCE & 76 \\
5 & - & $\mathrm{Ag}_{2} \mathrm{CO}_{3}$ & $\mathrm{~K}_{2} \mathrm{~S}_{2} \mathrm{O}_{8}$ & DCE & trace \\
6 & $\mathrm{Cu}_{2} \mathrm{O}$ & - & $\mathrm{K}_{2} \mathrm{~S}_{2} \mathrm{O}_{8}$ & DCE & trace \\
7 & $\mathrm{Cu}_{2} \mathrm{O}$ & $\mathrm{Ag}_{2} \mathrm{CO}_{3}$ & - & DCE & 21 \\
\hline
\end{tabular}

${ }^{\text {a }}$ Reaction conditions: $4 \mathbf{a}(0.2 \mathrm{mmol}), \mathbf{2 a}\left(2.0\right.$ equiv), $\mathrm{Cu}_{2} \mathrm{O}$ (10 mol \%), $\mathrm{Ag}_{2} \mathrm{CO}_{3}(20 \mathrm{~mol} \%), \mathrm{K}_{2} \mathrm{~S}_{2} \mathrm{O}_{8}$ (2.0 equiv), DCE (1.0 mL), $80{ }^{\circ} \mathrm{C}, 3 \mathrm{~h}$, air. ${ }^{\mathrm{b}}$ Isolated yields. ${ }^{\mathrm{c}} 1$ equivalent of $\mathrm{PhSO}_{2} \mathrm{Na}$ was used. ${ }^{\mathrm{d}} 1.5$ equivalents of $\mathrm{PhSO}_{2} \mathrm{Na}$ was used. ${ }^{\text {e }} 2.5$ equivalent of $\mathrm{PhSO}_{2} \mathrm{Na}$ was used. 


\section{Mechanism Investigation}

Figure S1. Investigation of the Oxidation State of Copper Catalyst

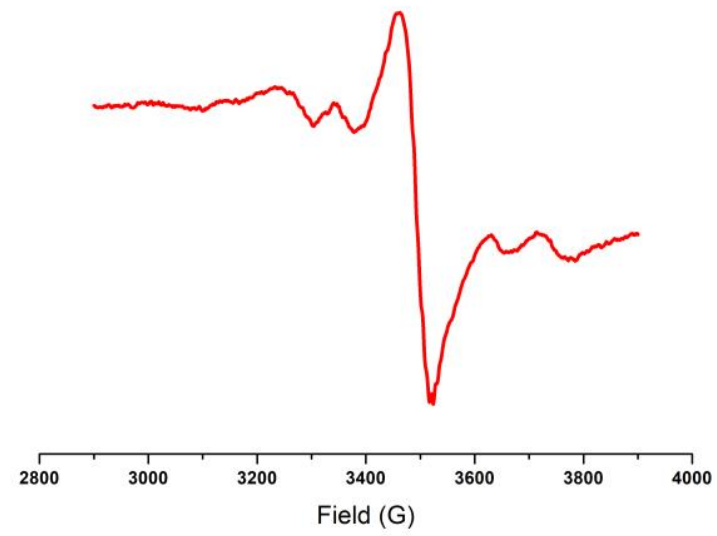

The reaction was monitored by electron paramagnetic resonance (EPR). As shown in Figure S1, when the reaction was performed for $5 \mathrm{~min}$, the EPR signal of $\mathrm{Cu}(\mathrm{II})$ appears, suggesting its formation in the reaction mixture (Dalton Trans. 2018, 47, 15024).

Scheme S2. ${ }^{18} \mathrm{O}$-Labeling Experiments
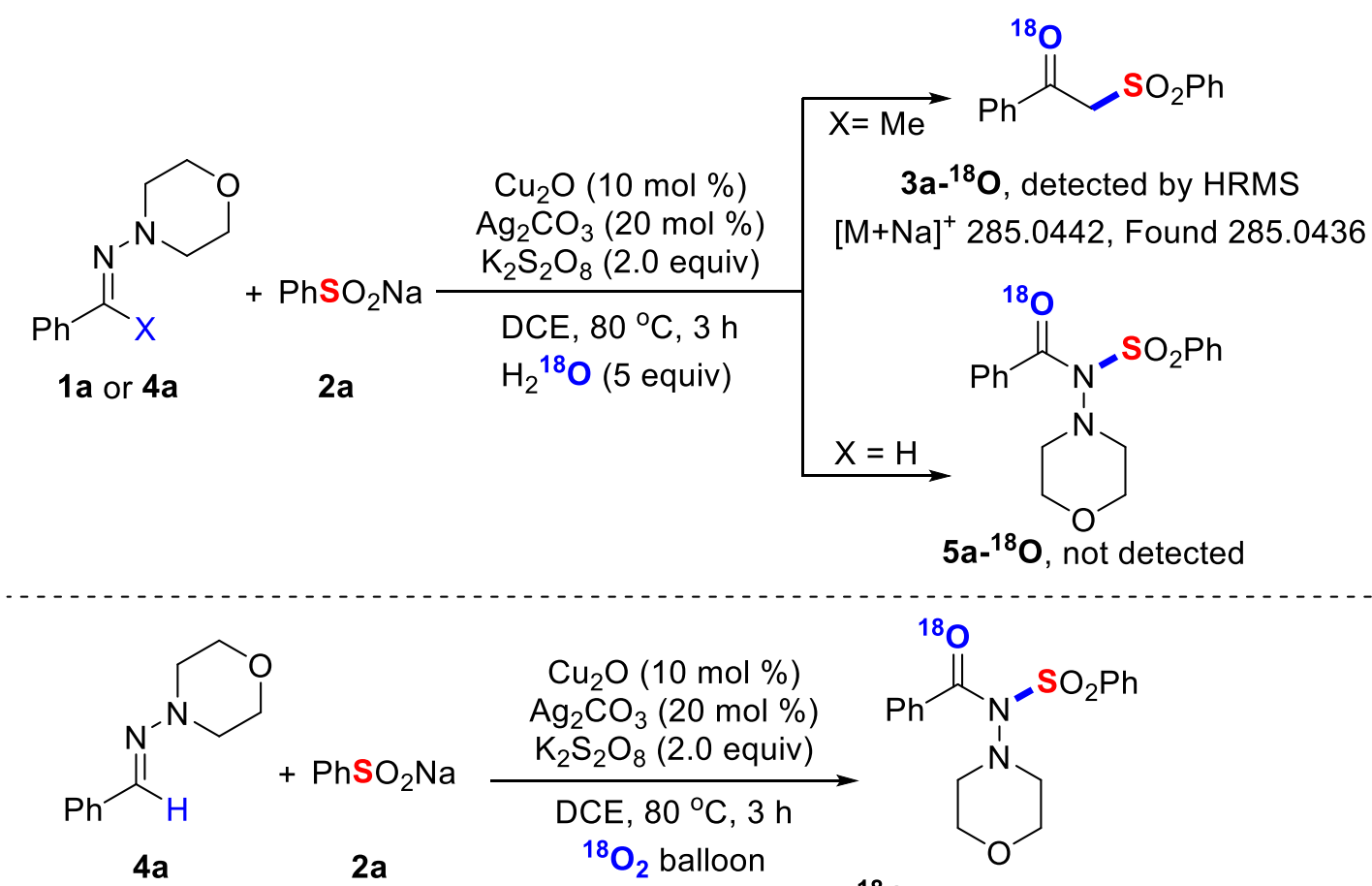

$5 \mathbf{a}^{-18} \mathbf{O}$, detected by HRMS

$[\mathrm{M}+\mathrm{Na}]^{+}$371.0922, Found 371.0924 
Scheme S3. Investigation of Influence of Atmosphere on the Reactions

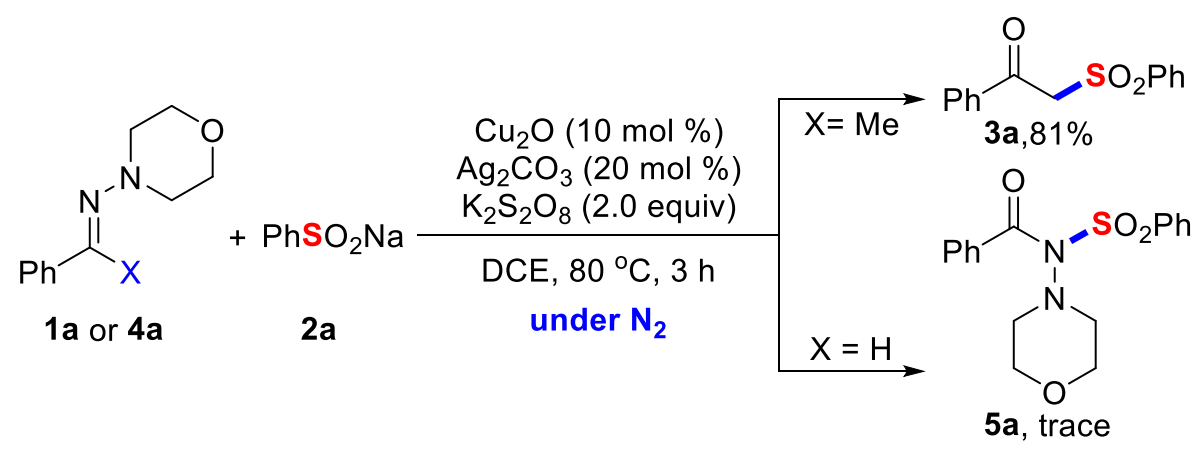




\section{X-ray Crystal Data for 3g and $5 n$}

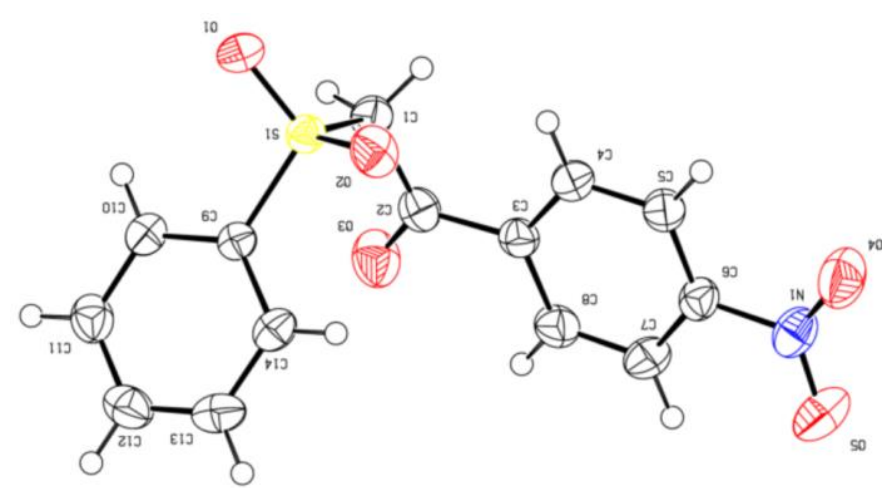

Figure S2 Single-crystal X-Ray structure of 3g. Ellipsoids are represented at 30\% Probability.

Table S10. Crystallographic Data and Structure Refinement for 3g

\begin{tabular}{|c|c|}
\hline $\mathrm{CCDC}$ & 1902707 \\
\hline Empirical formula & $\mathrm{C}_{14} \mathrm{H}_{11} \mathrm{NO}_{5} \mathrm{~S}$ \\
\hline Formula weight & 305.30 \\
\hline Temperature K & 296.15 \\
\hline Wavelength $\AA$ & 0.71073 \\
\hline Crystal system & Monoclinic \\
\hline Space group & P $121 /$ n 1 \\
\hline$a, b, c \AA$ & $12.7974(19), 5.3455(8), 20.549$ (3) \\
\hline$\alpha, \beta, \gamma^{\mathrm{o}}$ & $90,107.792(2), 90$ \\
\hline Volume $\AA^{3}$ & $1338.5(3)$ \\
\hline$Z$ & 4 \\
\hline Calculated density, $\mathrm{Mg} / \mathrm{m}^{\wedge} 3$ & 1.515 \\
\hline$F(000)$ & 632 \\
\hline Theta range for data collection ${ }^{\circ}$ & 3.059 to 27.614 \\
\hline Limiting indices & $-14<=\mathrm{h}<=16,-6<=\mathrm{k}<=6,-25<=1<=26$ \\
\hline Reflections collected / unique & $7637 / 3049[\mathrm{R}($ int $)=0.0153]$ \\
\hline Absorption correction & Semi-empirical from equivalents \\
\hline Refinement method & Full-matrix least-squares on $\mathrm{F}^{2}$ \\
\hline Data / restraints / parameters & 3049 / 0 / 190 \\
\hline Goodness of fit on $F^{2}$ & 1.075 \\
\hline
\end{tabular}




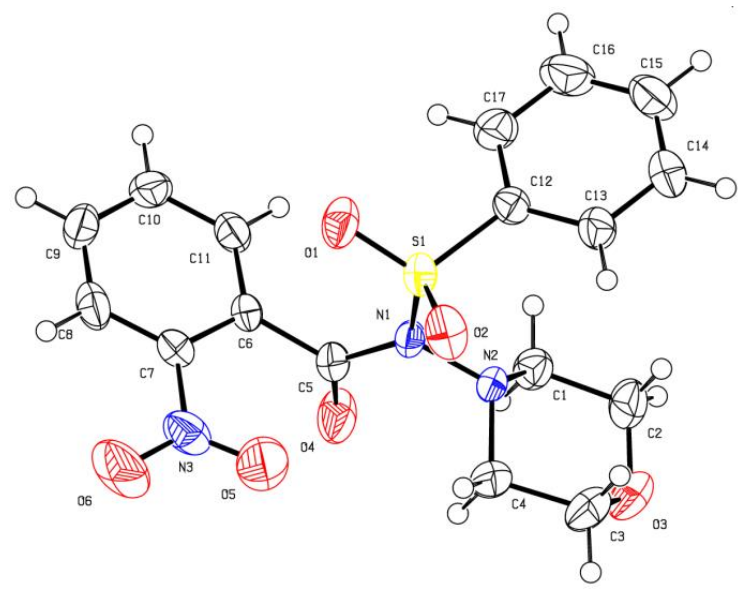

Figure S3 Single-crystal X-Ray structure of 5n. Ellipsoids are represented at 30\% Probability.

Table S11. Crystallographic Data and Structure Refinement for 5n

\begin{tabular}{|c|c|}
\hline CCDC & 2032258 \\
\hline Empirical formula & $\mathrm{C}_{17} \mathrm{H}_{17} \mathrm{~N}_{3} \mathrm{O}_{6} \mathrm{~S}$ \\
\hline Formula weight & 391.39 \\
\hline Temperature $\mathrm{K}$ & 296.15 \\
\hline Wavelength $\AA$ & 0.71073 \\
\hline Crystal system & Monoclinic \\
\hline Space group & P $121 / \mathrm{c} 1$ \\
\hline$a, b, c \AA$ & $7.6019(8), 20.085(2), 11.8715(13)$ \\
\hline$\alpha, \beta, \gamma^{\circ}$ & $90,104.312(2), 90$ \\
\hline Volume $\AA^{3}$ & $1756.3(3)$ \\
\hline$Z$ & 4 \\
\hline Calculated density, $\mathrm{Mg} / \mathrm{m}^{\wedge} 3$ & 1.480 \\
\hline$F(000)$ & 816 \\
\hline Theta range for data collection ${ }^{\circ}$ & 2.692 to 27.705 \\
\hline Limiting indices & $-8<=\mathrm{h}<=9,-25<=\mathrm{k}<=26,-13<=1<=14$ \\
\hline Reflections collected / unique & $10542 / 4023[\mathrm{R}(\mathrm{int})=0.0212]$ \\
\hline Absorption correction & Semi-empirical from equivalents \\
\hline Refinement method & Full-matrix least-squares on $\mathrm{F}^{2}$ \\
\hline Data / restraints / parameters & 4023 / 0 / 244 \\
\hline Goodness of fit on $F^{2}$ & 1.014 \\
\hline
\end{tabular}




\section{Copies of NMR and HRMS Spectra}

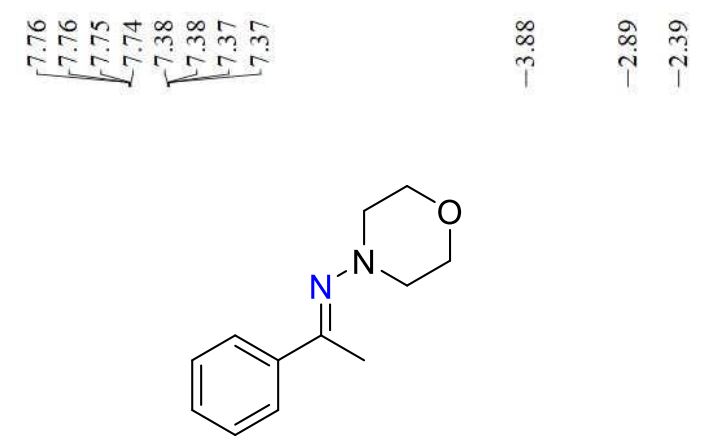

${ }^{1} \mathrm{H}$ NMR $\left(\mathrm{CDCl}_{3}, 500 \mathrm{MHz}\right)$

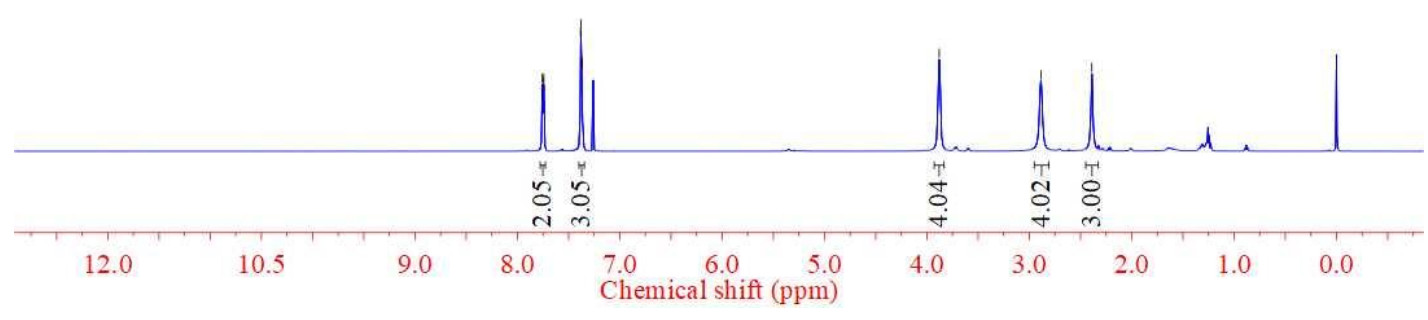

${ }^{1} \mathrm{H}$ NMR Spectrum of Compound 1a

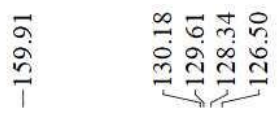

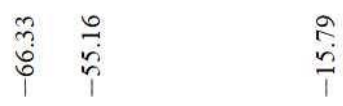<smiles>C/C(=N\N1CCOCC1)c1ccccc1</smiles>

${ }^{13} \mathrm{C}\left\{{ }^{1} \mathrm{H}\right\}$ NMR $\left(\mathrm{CDCl}_{3}, 126 \mathrm{MHz}\right)$

$210 \quad 190$

130

110

$90 \quad 80$

${ }^{13} \mathbf{C}\left\{{ }^{1} \mathrm{H}\right\}$ NMR Spectrum of Compound 1a 
过苛究<smiles>C/C(=N\N1CCOCC1)c1ccc(C)cc1</smiles>

${ }^{1} \mathrm{H}$ NMR $\left(\mathrm{CDCl}_{3}, 500 \mathrm{MHz}\right)$

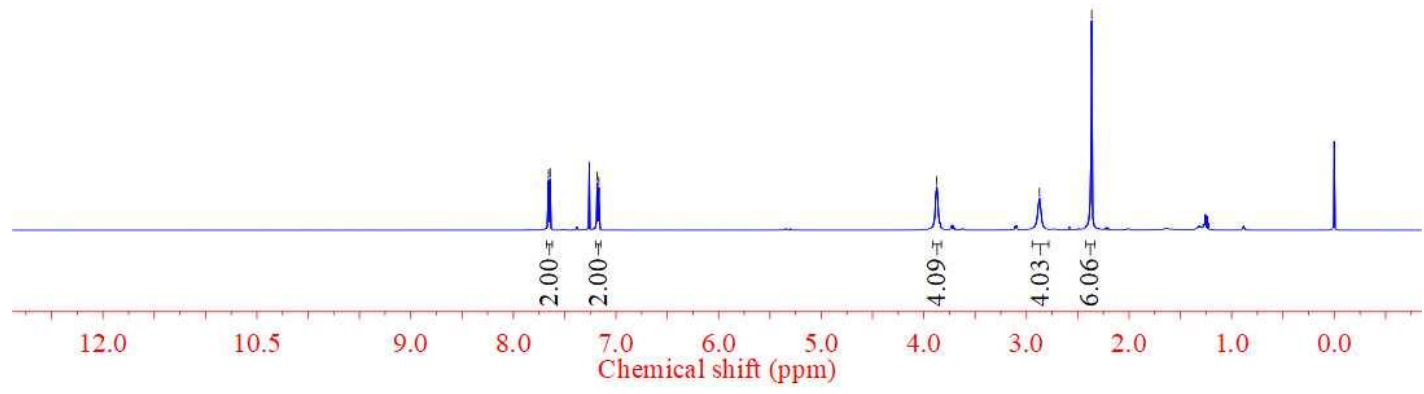

${ }^{1}$ H NMR Spectrum of Compound $1 \mathrm{~b}$

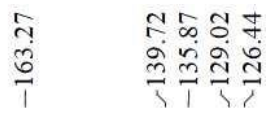

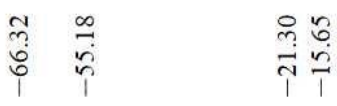<smiles>C/C(=N\N1CCOCC1)c1ccc(C)cc1</smiles>

${ }^{13} \mathrm{C}\left\{{ }^{1} \mathrm{H}\right\}$ NMR $\left(\mathrm{CDCl}_{3}, 126 \mathrm{MHz}\right)$

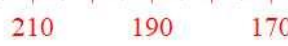

150

${ }^{13} \mathrm{C}\left\{{ }^{1} \mathrm{H}\right\}$ NMR Spectrum of Compound $1 \mathrm{~b}$ 


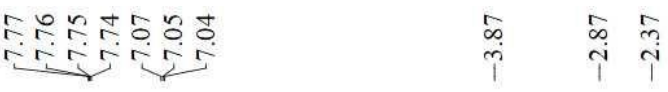<smiles>C/C(=N\N1CCOCC1)c1ccc(F)cc1</smiles>

${ }^{1} \mathrm{H}$ NMR (CDCl3, $\left.500 \mathrm{MHz}\right)$

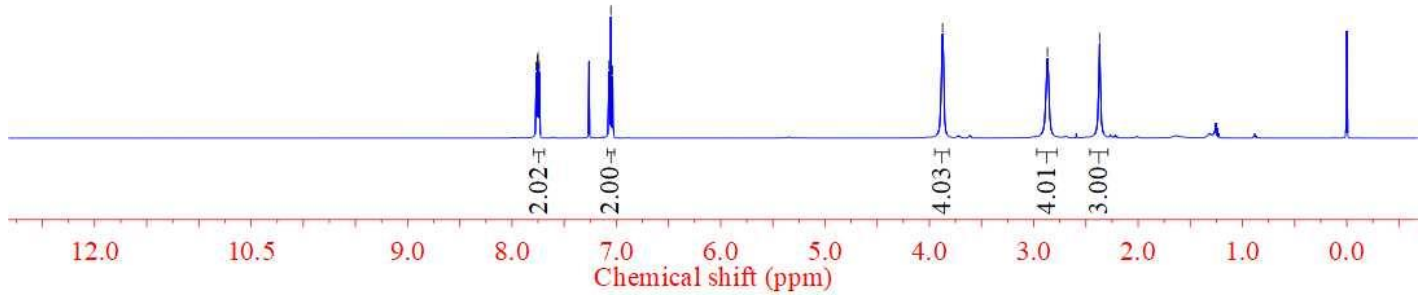

${ }^{1}$ H NMR Spectrum of Compound 1c

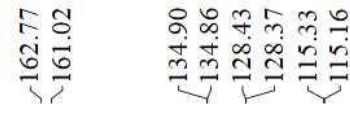<smiles>C/C(=N\N1CCOCC1)c1ccc(F)cc1</smiles>

${ }^{13} \mathrm{C}\left\{{ }^{1} \mathrm{H}\right\} \mathrm{NMR}(\mathrm{CDCl} 3,126 \mathrm{MHz})$

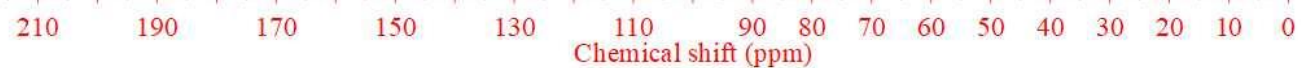

${ }^{13} \mathbf{C}\left\{{ }^{1} \mathbf{H}\right\}$ NMR Spectrum of Compound 1c 
<smiles>C/C(=N\N1CCOCC1)c1ccc(Cl)cc1</smiles>

${ }^{1} \mathrm{H}$ NMR (CDCl $\left.3,500 \mathrm{MHz}\right)$

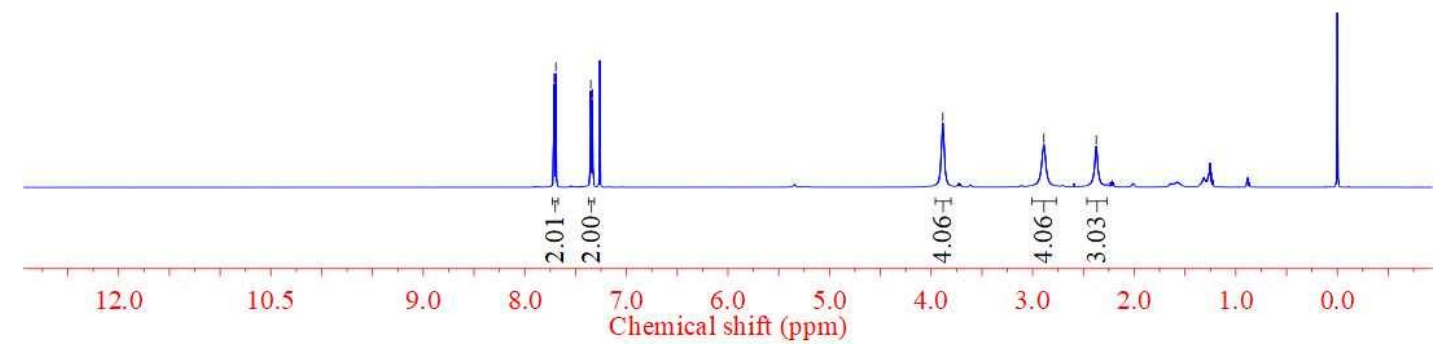

${ }^{1}$ H NMR Spectrum of Compound 1d

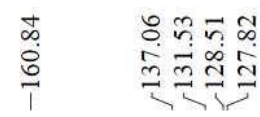

$\begin{array}{lll}0 & n & \text { 웅 } \\ 0 & \text { in } & \text { in }\end{array}$<smiles>C/C(=N\N1CCOCC1)c1ccc(Cl)cc1</smiles>

${ }^{13} \mathrm{C}\left\{{ }^{1} \mathrm{H}\right\}$ NMR $\left(\mathrm{CDCl}_{3}, 126 \mathrm{MHz}\right)$

${ }^{13} \mathbf{C}\left\{{ }^{1} \mathrm{H}\right\}$ NMR Spectrum of Compound 1d 
<smiles>C/C(=N\N1CCOCC1)c1ccc(Br)cc1</smiles>

${ }^{1} \mathrm{H}$ NMR $\left(\mathrm{CDCl}_{3}, 500 \mathrm{MHz}\right)$

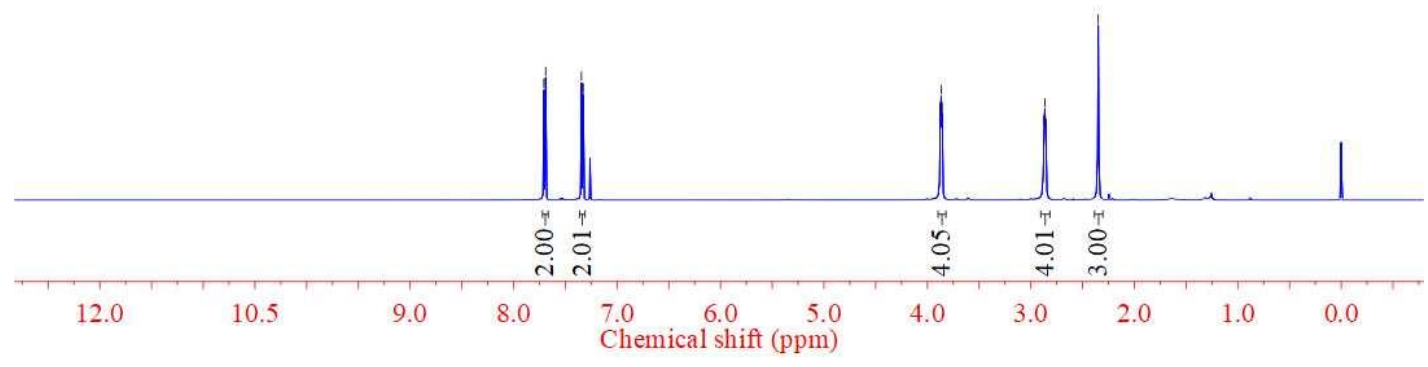

${ }^{1}$ H NMR Spectrum of Compound 1e

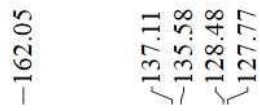

$\begin{array}{ll}\vec{m} & 0 \\ 0 & 0 \\ i & i\end{array}$

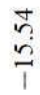<smiles>C/C(=N\N1CCOCC1)c1ccc(Br)cc1</smiles>

${ }^{13} \mathrm{C}\left\{{ }^{1} \mathrm{H}\right\}$ NMR (CDCl3, $\left.126 \mathrm{MHz}\right)$

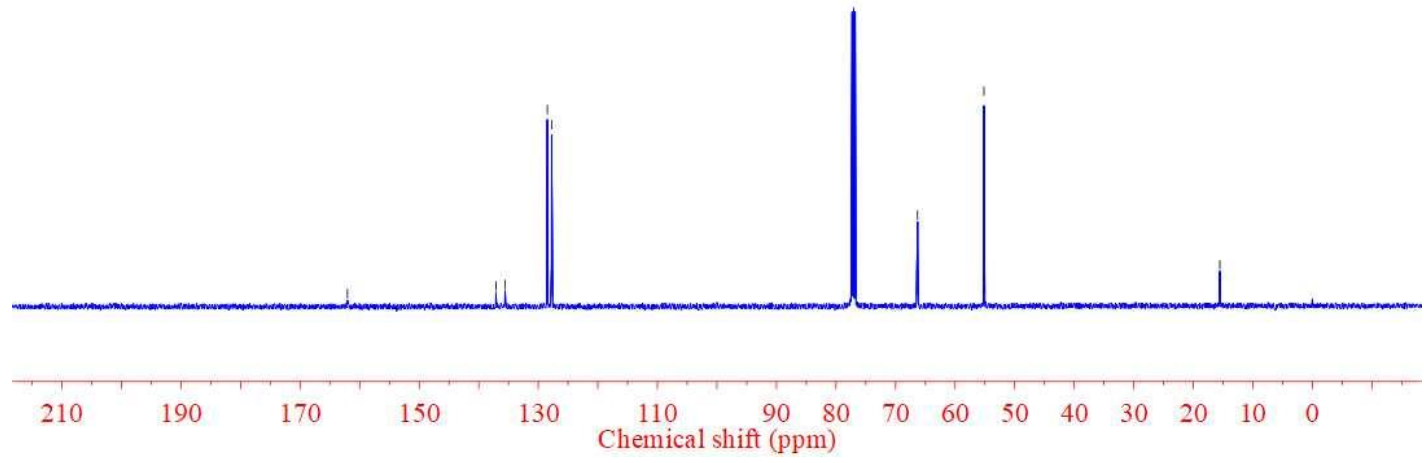

${ }^{13} \mathrm{C}\left\{{ }^{1} \mathrm{H}\right\}$ NMR Spectrum of Compound 1e 
<smiles>C/C(=N\N1CCOCC1)c1ccc(I)cc1</smiles>

${ }^{1} \mathrm{H}$ NMR (CDCl3, $\left.500 \mathrm{MHz}\right)$

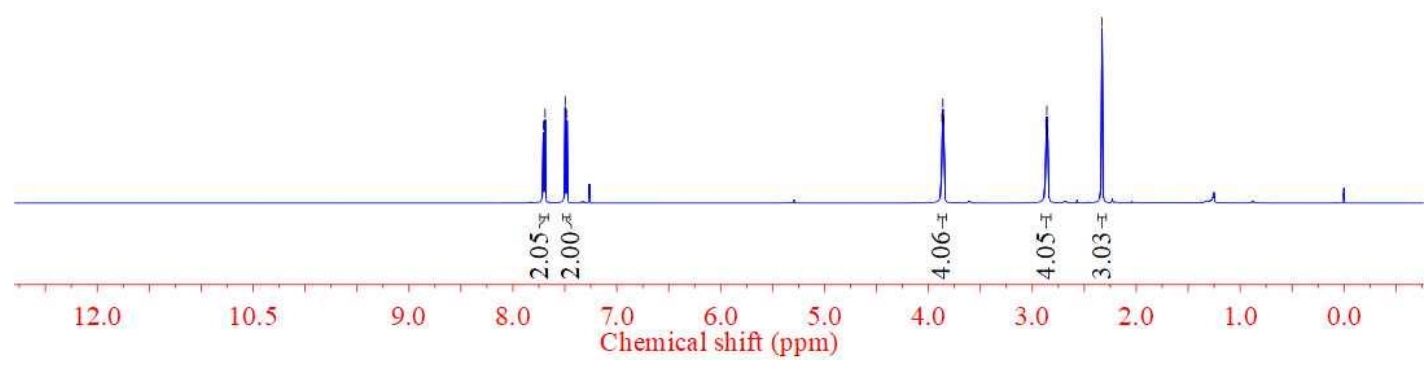

${ }^{1}$ H NMR Spectrum of Compound 1f

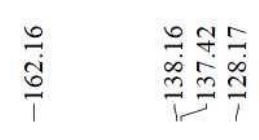<smiles>CC(=O)N1CCOCC1</smiles>

${ }^{13} \mathrm{C}\left\{{ }^{1} \mathrm{H}\right\} \mathrm{NMR}\left(\mathrm{CDCl}_{3}, 126 \mathrm{MHz}\right)$

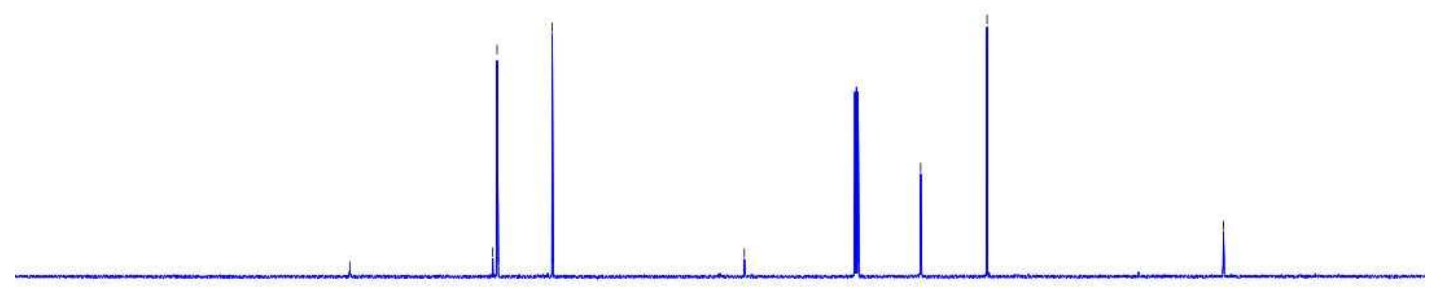

$210 \quad 190 \quad 170$

${ }^{13} \mathrm{C}\left\{{ }^{1} \mathrm{H}\right\}$ NMR Spectrum of Compound 1f 
<smiles>C/C(=N\N1CCOCC1)c1ccc([N+](=O)[O-])cc1</smiles>

${ }^{1} \mathrm{H} \mathrm{NMR}\left(\mathrm{CDCl}_{3}, 500 \mathrm{MHz}\right)$

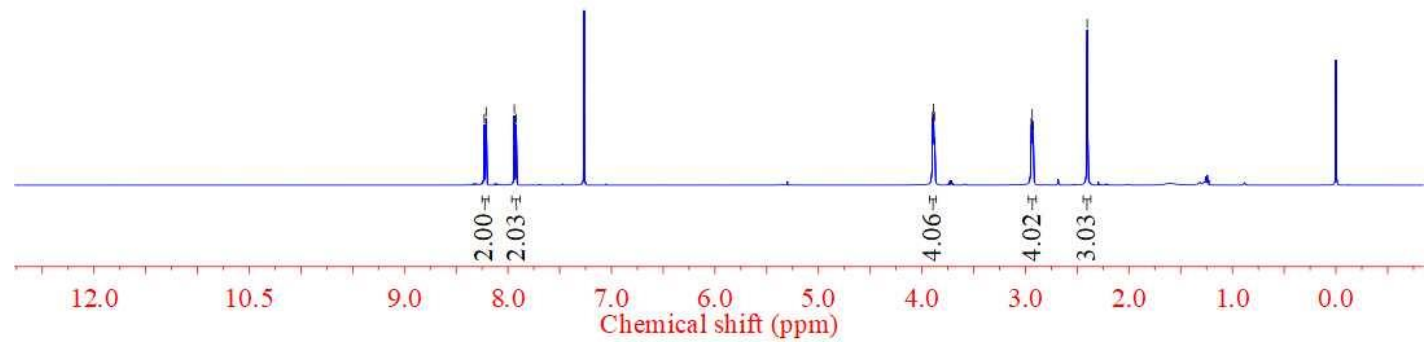

${ }^{1} \mathrm{H}$ NMR Spectrum of Compound $1 \mathrm{~g}$

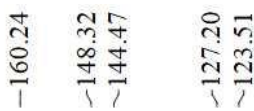

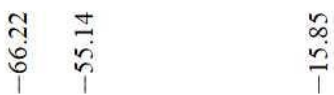<smiles>C/C(=N\N1CCOCC1)c1ccc([N+](=O)[O-])cc1</smiles>

${ }^{13} \mathrm{C}\left\{{ }^{1} \mathrm{H}\right\} \mathrm{NMR}\left(\mathrm{CDCl}_{3}, 126 \mathrm{MHz}\right)$ 
<smiles>C/C(=N\N1CCOCC1)c1cccc(C)c1</smiles>

${ }^{1} \mathrm{H}$ NMR (CDCl3, $\left.500 \mathrm{MHz}\right)$

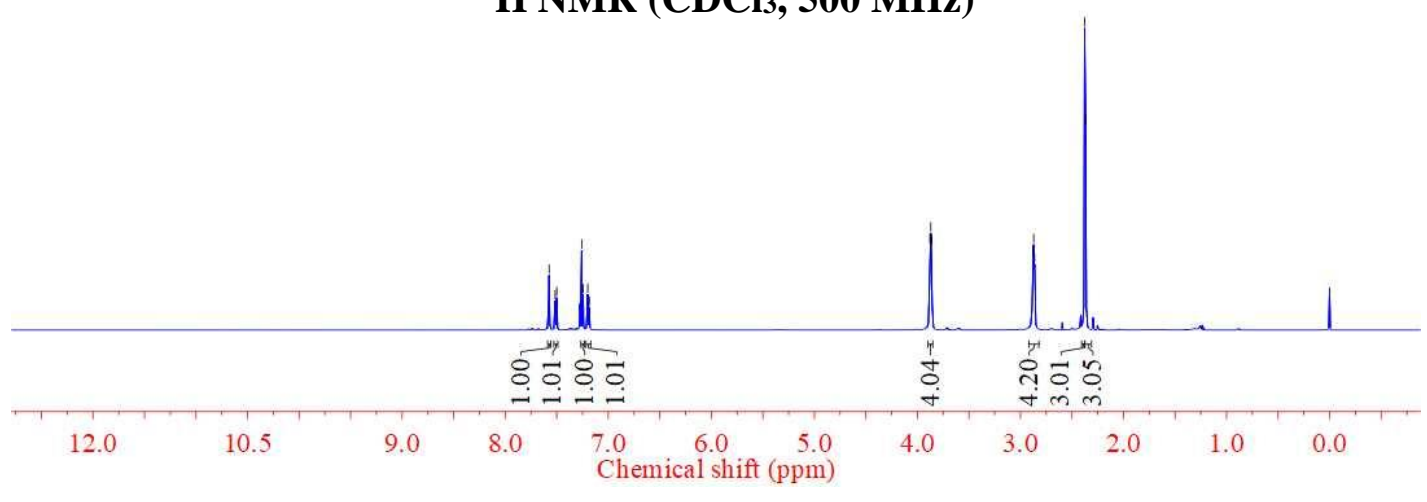

${ }^{1} \mathrm{H}$ NMR Spectrum of Compound $1 \mathrm{~h}$

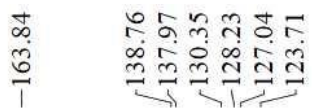

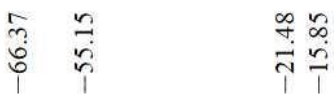<smiles>C/C(=N\N1CCOCC1)c1cccc(C)c1</smiles>

${ }^{13} \mathrm{C}\left\{{ }^{1} \mathrm{H}\right\} \mathrm{NMR}\left(\mathrm{CDCl}_{3}, 126 \mathrm{MHz}\right)$

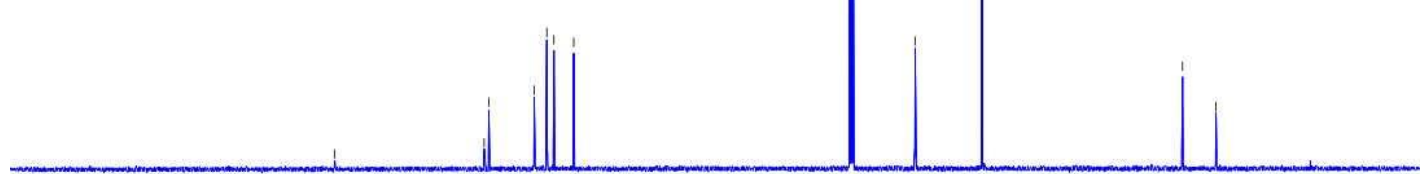

${ }^{13} \mathrm{C}\left\{{ }^{1} \mathrm{H}\right\}$ NMR Spectrum of Compound $1 \mathrm{~h}$ 
<smiles>C/C(=N\N1CCOCC1)c1cccc(F)c1</smiles>

${ }^{1} \mathrm{H}$ NMR $\left(\mathrm{CDCl}_{3}, 500 \mathrm{MHz}\right)$

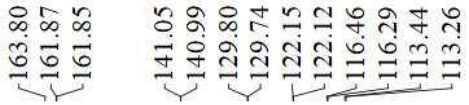

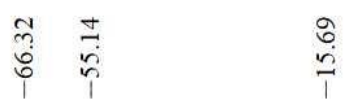<smiles>C/C(=N\N1CCOCC1)c1cccc(F)c1</smiles>

${ }^{13} \mathrm{C}\left\{{ }^{1} \mathrm{H}\right\}$ NMR $\left(\mathrm{CDCl}_{3}, 126 \mathrm{MHz}\right)$

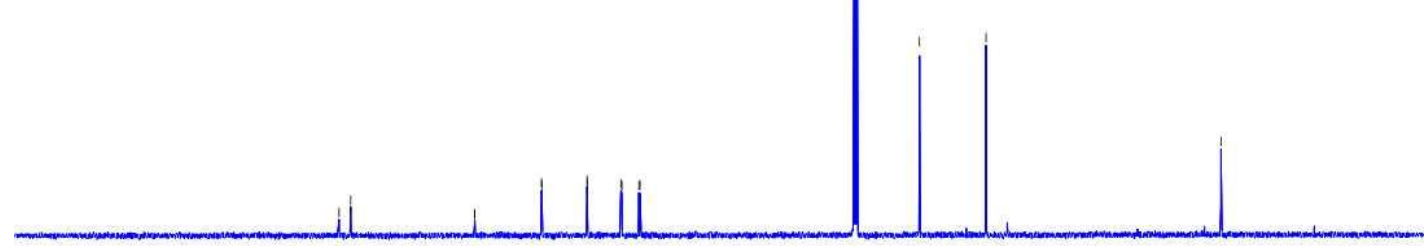


<smiles>C/C(=N\N1CCOCC1)c1cccc(Cl)c1</smiles>

${ }^{1} \mathrm{H}$ NMR (CDCl3, $\left.500 \mathrm{MHz}\right)$

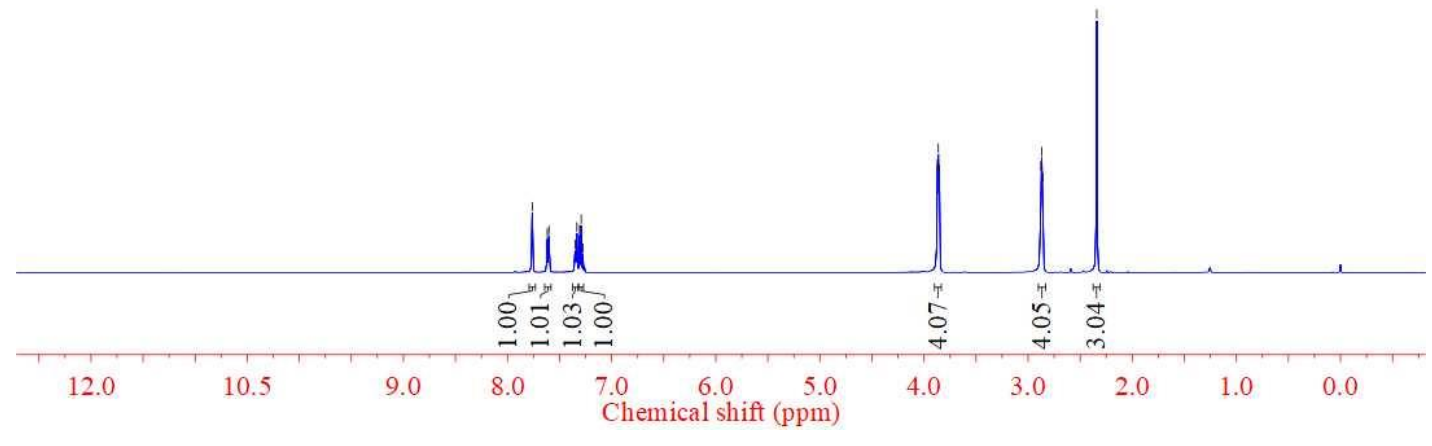

${ }^{1}$ H NMR Spectrum of Compound $\mathbf{1 j}$

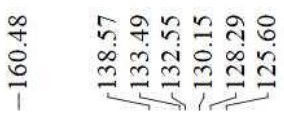

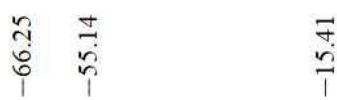<smiles>C/C(=N\N1CCOCC1)c1cccc(Cl)c1</smiles>

${ }^{13} \mathrm{C}\left\{{ }^{1} \mathrm{H}\right\} \mathrm{NMR}\left(\mathrm{CDCl}_{3}, 126 \mathrm{MHz}\right)$

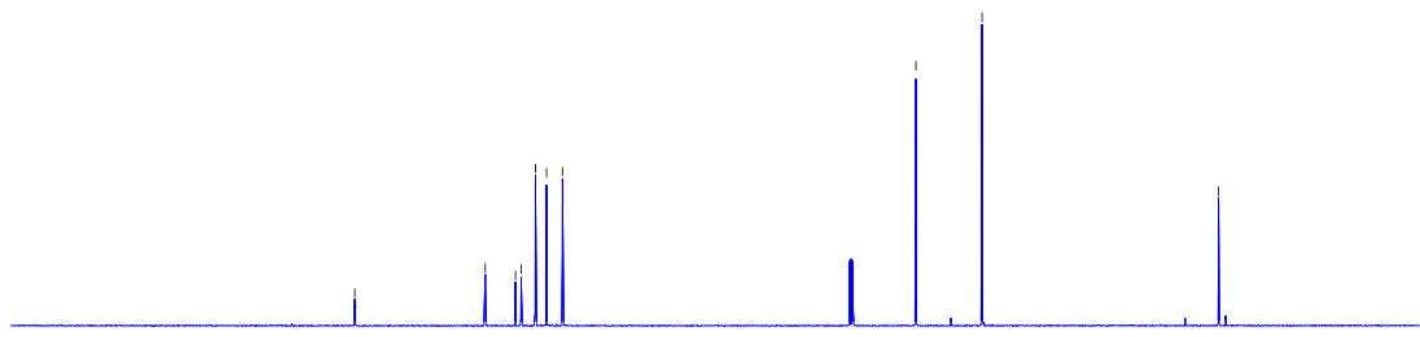

\section{${ }^{13} \mathbf{C}\left\{{ }^{1} \mathrm{H}\right\}$ NMR Spectrum of Compound $1 \mathbf{j}$}


<smiles>C/C(=N\N1CCOCC1)c1cccc(Br)c1</smiles>

${ }^{1} \mathrm{H}$ NMR $\left(\mathrm{CDCl}_{3}, 500 \mathrm{MHz}\right)$

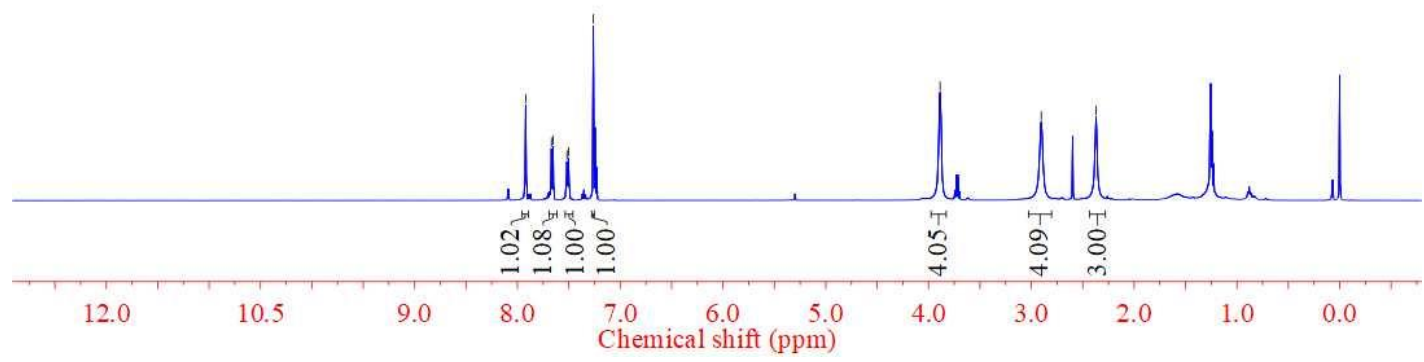

${ }^{1}$ H NMR Spectrum of Compound 1k

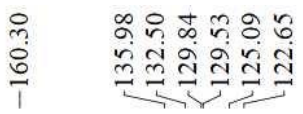

तु<smiles>C/C(=N\N1CCOCC1)c1cccc(Br)c1</smiles>

${ }^{13} \mathrm{C}\left\{{ }^{1} \mathrm{H}\right\}$ NMR $\left(\mathrm{CDCl}_{3}, 126 \mathrm{MHz}\right)$ 
<smiles>C/C(=N\N1CCOCC1)c1cccc(C(F)(F)F)c1</smiles>

${ }^{1} \mathrm{H}$ NMR $\left(\mathrm{CDCl}_{3}, 500 \mathrm{MHz}\right)$
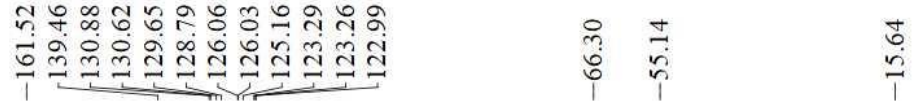<smiles>C/C(=N\N1CCOCC1)c1cccc(C(F)(F)F)c1</smiles>

${ }^{13} \mathrm{C}\left\{{ }^{1} \mathrm{H}\right\} \mathrm{NMR}\left(\mathrm{CDCl}_{3}, 126 \mathrm{MHz}\right)$

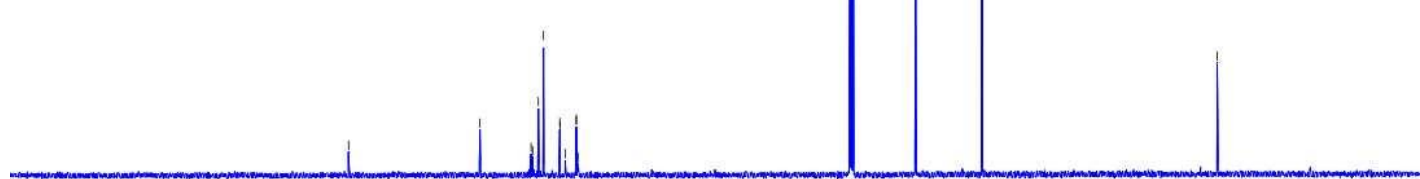


<smiles>C/C(=N\N1CCOCC1)c1ccccc1C</smiles>

${ }^{1} \mathrm{H}$ NMR $\left(\mathrm{CDCl}_{3}, 500 \mathrm{MHz}\right)$

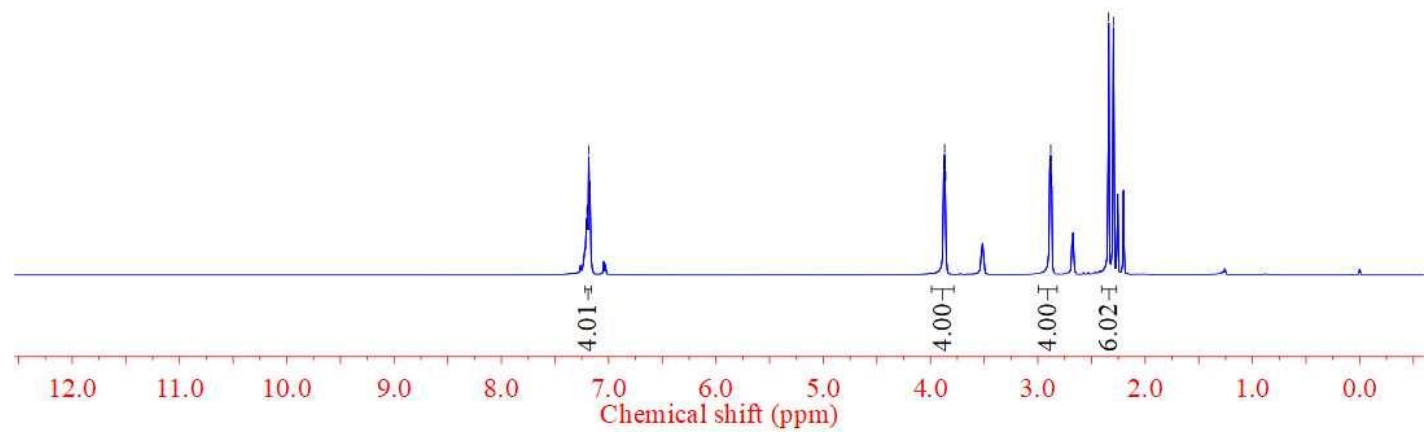

${ }^{1} \mathrm{H}$ NMR Spectrum of Compound $1 \mathrm{~m}$

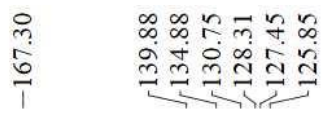

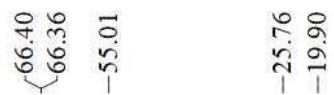<smiles>C/C(=N\N1CCOCC1)c1ccccc1C</smiles>

${ }^{13} \mathrm{C}\left\{{ }^{1} \mathrm{H}\right\}$ NMR $\left(\mathrm{CDCl}_{3}, 126 \mathrm{MHz}\right)$

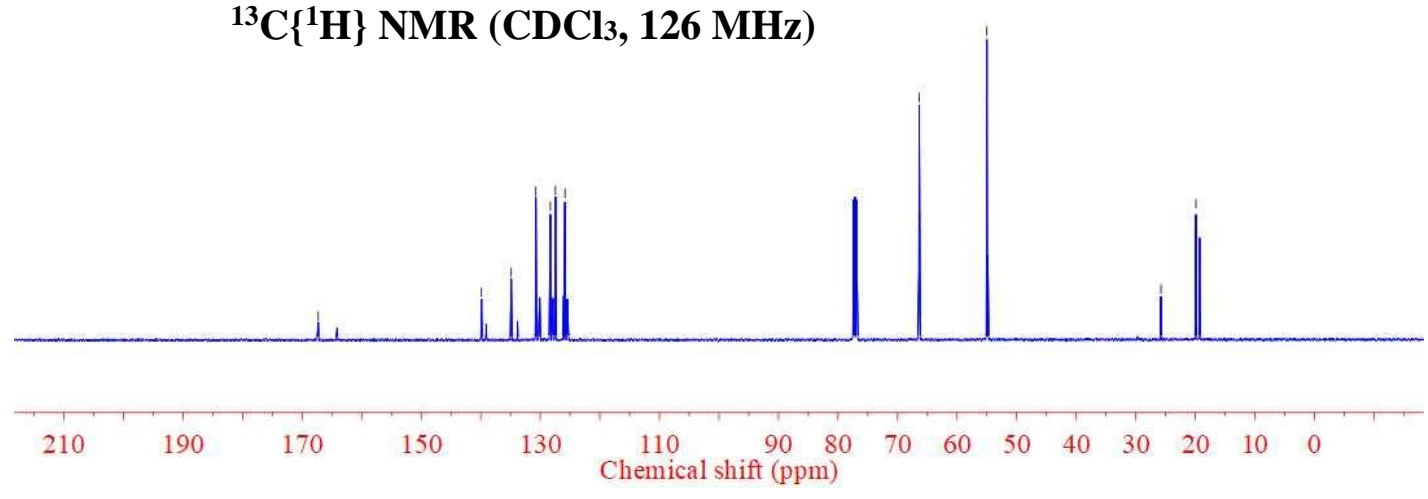

${ }^{13} \mathrm{C}\left\{{ }^{1} \mathrm{H}\right\}$ NMR Spectrum of Compound $1 \mathrm{~m}$ 
<smiles>C/C(=N\N1CCOCC1)c1ccccc1F</smiles>

${ }^{1} \mathrm{H}$ NMR $\left(\mathrm{CDCl}_{3}, 500 \mathrm{MHz}\right)$

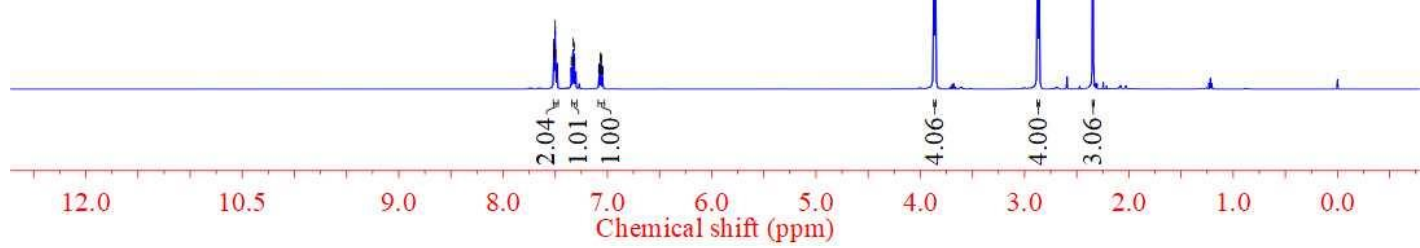

${ }^{1}$ H NMR Spectrum of Compound 1 n

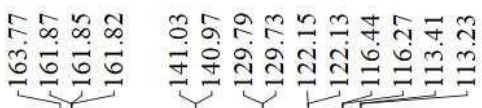

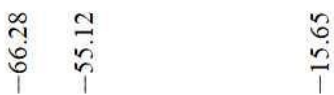<smiles>C/C(=N\N1CCOCC1)c1ccccc1F</smiles>

${ }^{13} \mathrm{C}\left\{{ }^{1} \mathrm{H}\right\}$ NMR $\left(\mathrm{CDCl}_{3}, 126 \mathrm{MHz}\right)$

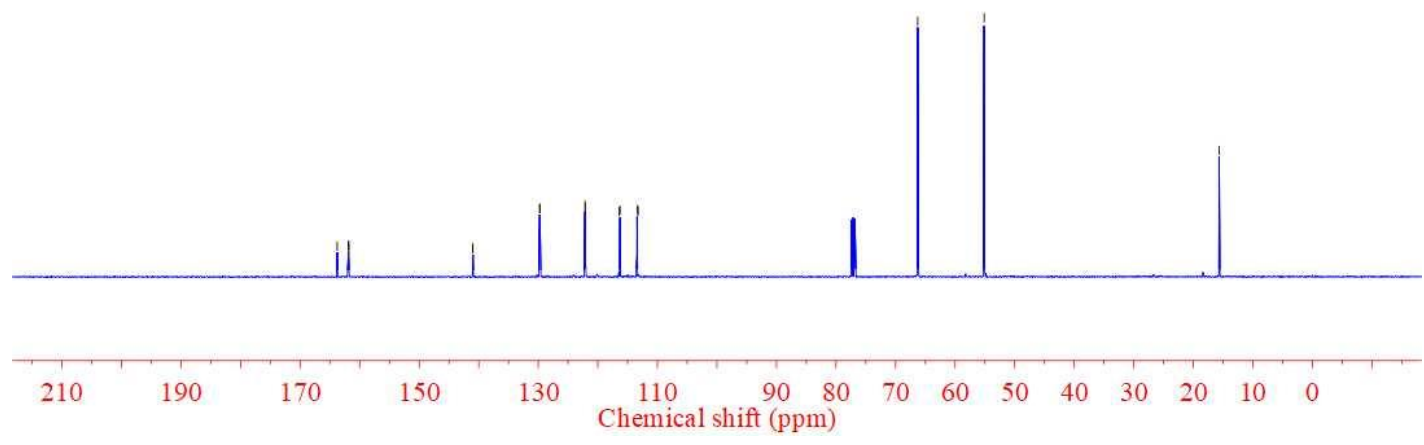

${ }^{13} \mathrm{C}\left\{{ }^{1} \mathrm{H}\right\}$ NMR Spectrum of Compound $1 \mathrm{n}$ 
<smiles>C/C(=N\N1CCOCC1)c1cc(F)ccc1F</smiles>

${ }^{1} \mathrm{H}$ NMR $\left(\mathrm{CDCl}_{3}, 500 \mathrm{MHz}\right)$

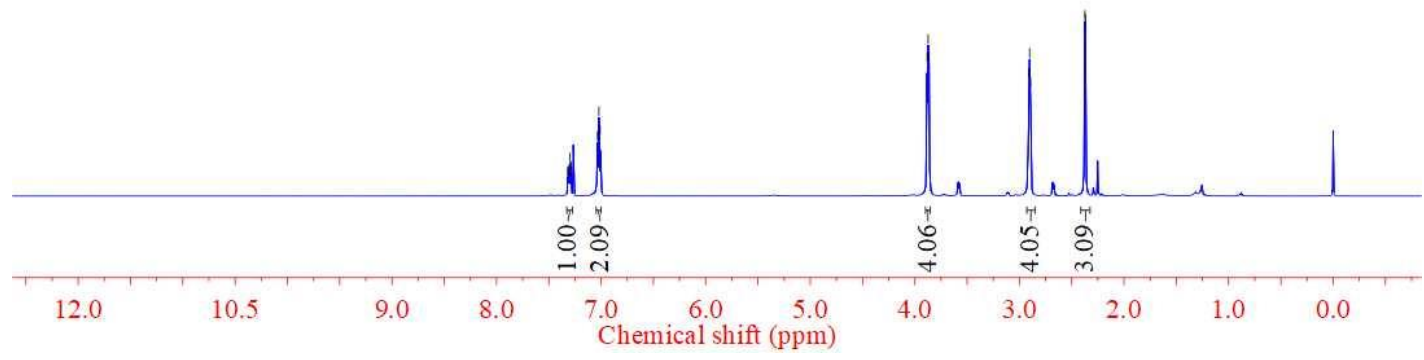

${ }^{1} \mathrm{H}$ NMR Spectrum of Compound 10

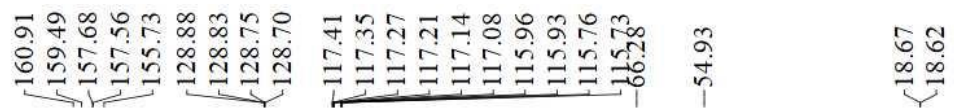<smiles>C/C(=N\N1CCOCC1)c1cc(F)ccc1F</smiles>

${ }^{13} \mathrm{C}\left\{{ }^{1} \mathrm{H}\right\}$ NMR $\left(\mathrm{CDCl}_{3}, 126 \mathrm{MHz}\right)$ 
<smiles>C/C(=N\N1CCOCC1)c1cc(F)cc(F)c1</smiles>

${ }^{1} \mathrm{H}$ NMR $\left(\mathrm{CDCl}_{3}, 500 \mathrm{MHz}\right)$

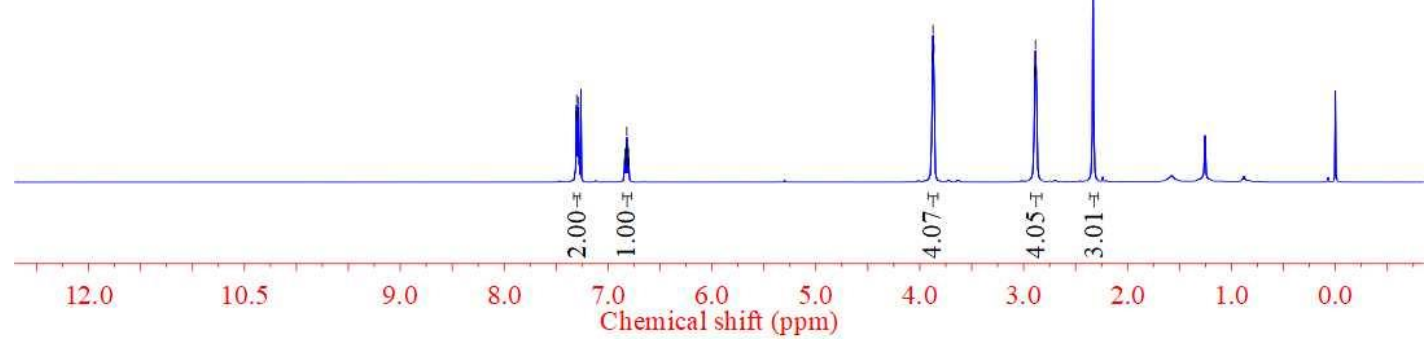

${ }^{1}$ H NMR Spectrum of Compound 1p

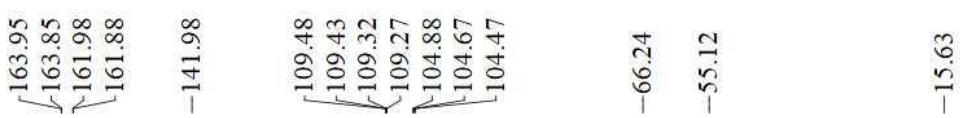<smiles>C/C(=N\N1CCOCC1)c1cc(F)cc(F)c1</smiles>

${ }^{13} \mathrm{C}\left\{{ }^{1} \mathrm{H}\right\}$ NMR $\left(\mathrm{CDCl}_{3}, 126 \mathrm{MHz}\right)$ 


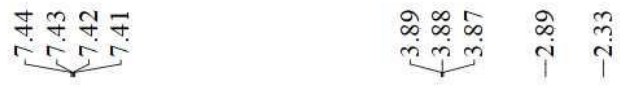<smiles>C/C(=N\N1CCOCC1)c1cc(F)c(F)c(F)c1</smiles>

${ }^{1} \mathrm{H}$ NMR $\left(\mathrm{CDCl}_{3}, 500 \mathrm{MHz}\right)$

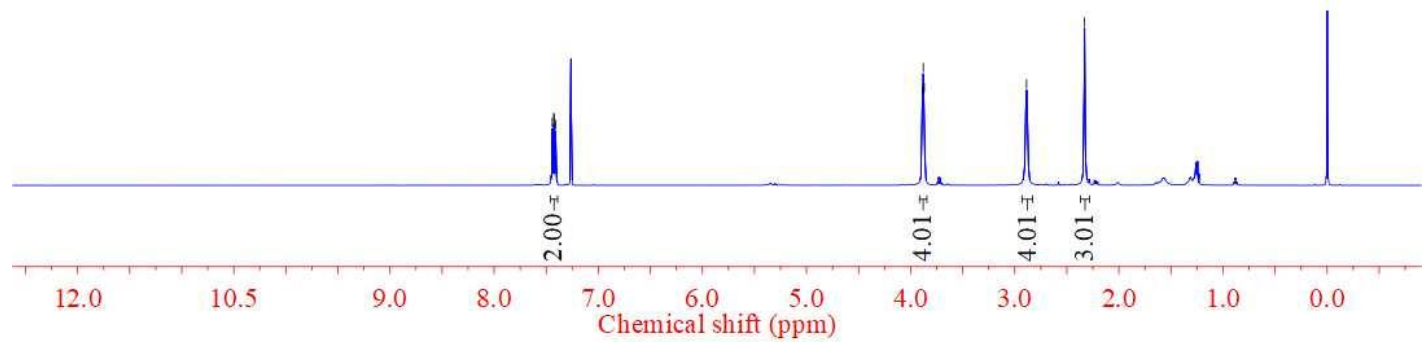

${ }^{1}$ H NMR Spectrum of Compound 1q

\begin{tabular}{|c|c|c|c|}
\hline 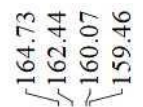 & 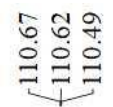 & $\begin{array}{l}\vec{N} \\
\ddot{\delta} \\
i\end{array}$ & $\frac{n}{n}$ \\
\hline
\end{tabular}<smiles>C/C(=N\N1CCOCC1)c1cc(F)c(F)c(F)c1</smiles>

${ }^{13} \mathrm{C}\left\{{ }^{1} \mathrm{H}\right\} \mathrm{NMR}(\mathrm{CDCl}, 126 \mathrm{MHz})$

$210190 \quad 170 \quad 150 \quad 130 \begin{gathered}110 \\ \text { Chemical shift }(\mathrm{ppm})\end{gathered}$

${ }^{13} \mathbf{C}\left\{{ }^{1} \mathbf{H}\right\}$ NMR Spectrum of Compound 1q 
<smiles>C/C(=N\N1CCOCC1)c1ccsc1</smiles>

${ }^{1} \mathrm{H}$ NMR $\left(\mathrm{CDCl}_{3}, 500 \mathrm{MHz}\right)$

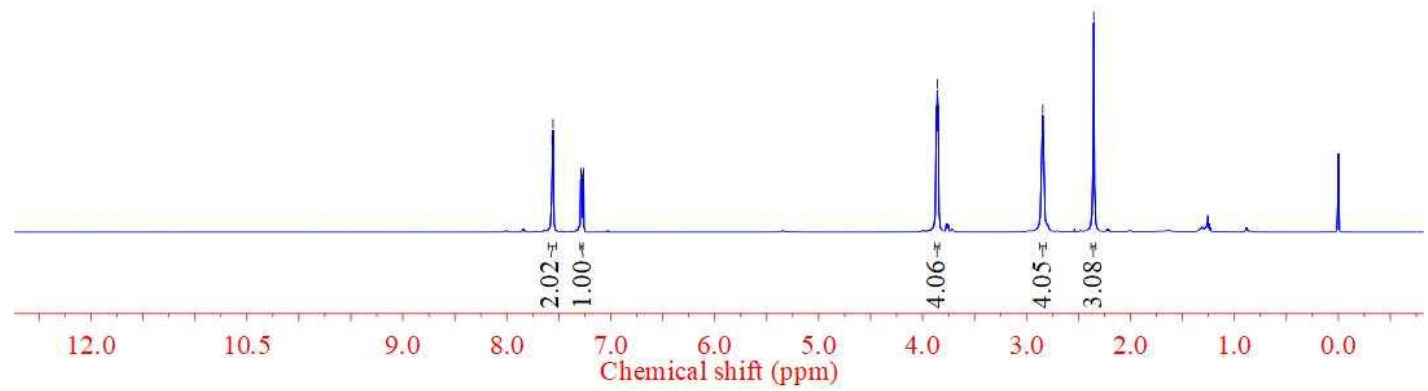

${ }^{1}$ H NMR Spectrum of Compound 1r

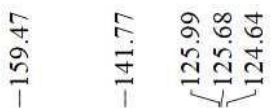

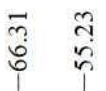

$\stackrel{8}{0}$<smiles>C/C(=N\N1CCOCC1)c1ccsc1</smiles>

${ }^{13} \mathrm{C}\left\{{ }^{1} \mathrm{H}\right\}$ NMR $\left(\mathrm{CDCl}_{3}, 126 \mathrm{MHz}\right)$ 
<smiles>CC/C(=N\N1CCOCC1)c1ccccc1</smiles>

${ }^{1} \mathrm{H}$ NMR $\left(\mathrm{CDCl}_{3}, 500 \mathrm{MHz}\right)$

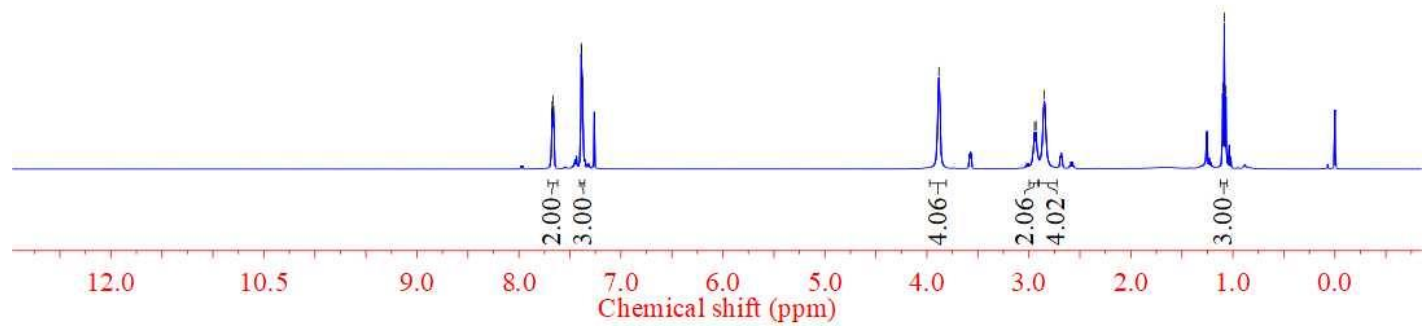

${ }^{1} \mathrm{H}$ NMR Spectrum of Compound 1s

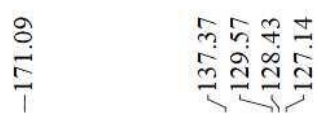

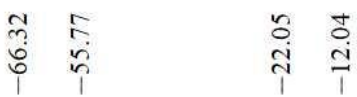<smiles>CC/C(=N\N1CCOCC1)c1ccccc1</smiles>

${ }^{13} \mathrm{C}\left\{{ }^{1} \mathrm{H}\right\}$ NMR $\left(\mathrm{CDCl}_{3}, 126 \mathrm{MHz}\right)$

$210 \quad 190 \quad 170$

150

130

Chemical shift (ppm)

${ }^{13} \mathrm{C}\left\{{ }^{1} \mathrm{H}\right\}$ NMR Spectrum of Compound 1s 


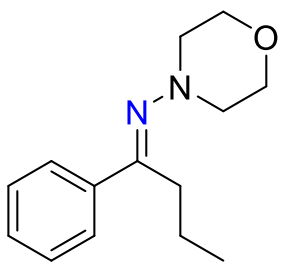

${ }^{1} \mathrm{H}$ NMR $\left(\mathrm{CDCl}_{3}, 500 \mathrm{MHz}\right)$

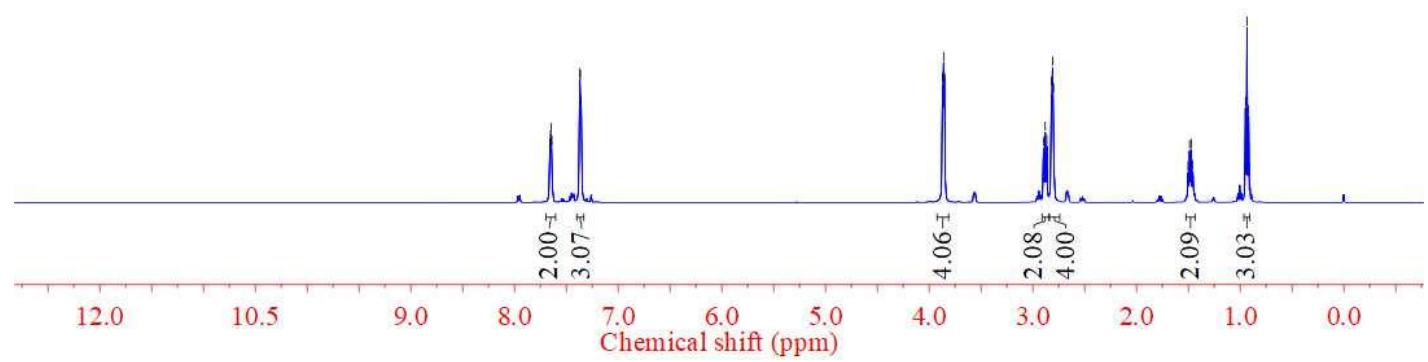

${ }^{1}$ H NMR Spectrum of Compound 1t

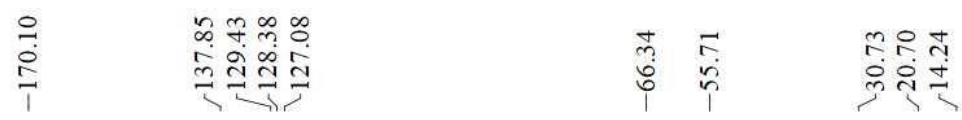<smiles>CCC/C(=N\N1CCOCC1)c1ccccc1</smiles>

${ }^{13} \mathrm{C}\left\{{ }^{1} \mathrm{H}\right\}$ NMR $\left(\mathrm{CDCl}_{3}, 126 \mathrm{MHz}\right)$

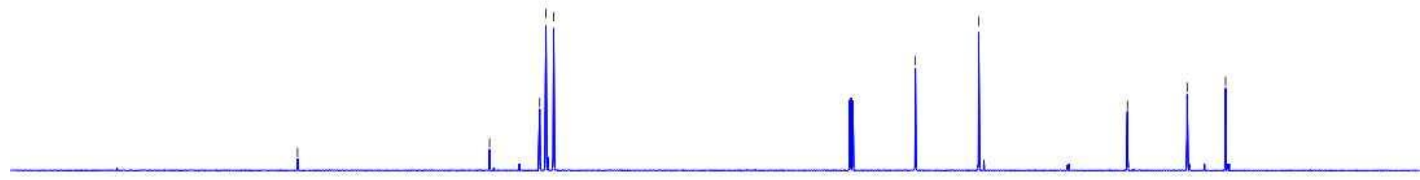


<smiles>CCCC/C(=N\N1CCOCC1)c1ccccc1</smiles>

${ }^{1} \mathrm{H}$ NMR $\left(\mathrm{CDCl}_{3}, 500 \mathrm{MHz}\right)$

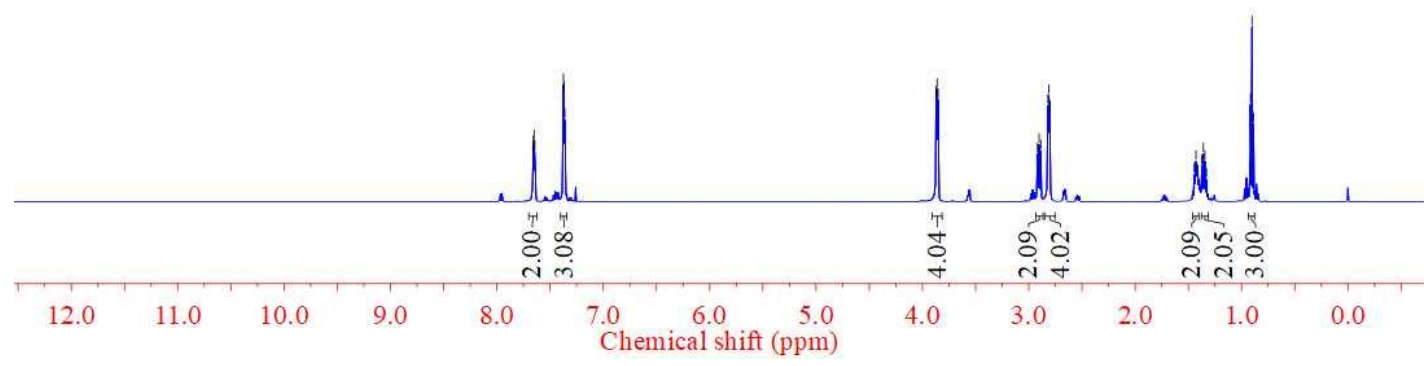

${ }^{1}$ H NMR Spectrum of Compound $1 u$

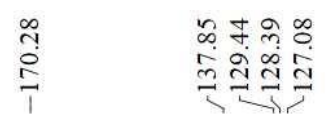

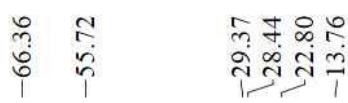<smiles>CCCC/C(=N\N1CCOCC1)c1ccccc1</smiles>

${ }^{13} \mathrm{C}\left\{{ }^{1} \mathrm{H}\right\} \mathrm{NMR}\left(\mathrm{CDCl}_{3}, 126 \mathrm{MHz}\right)$

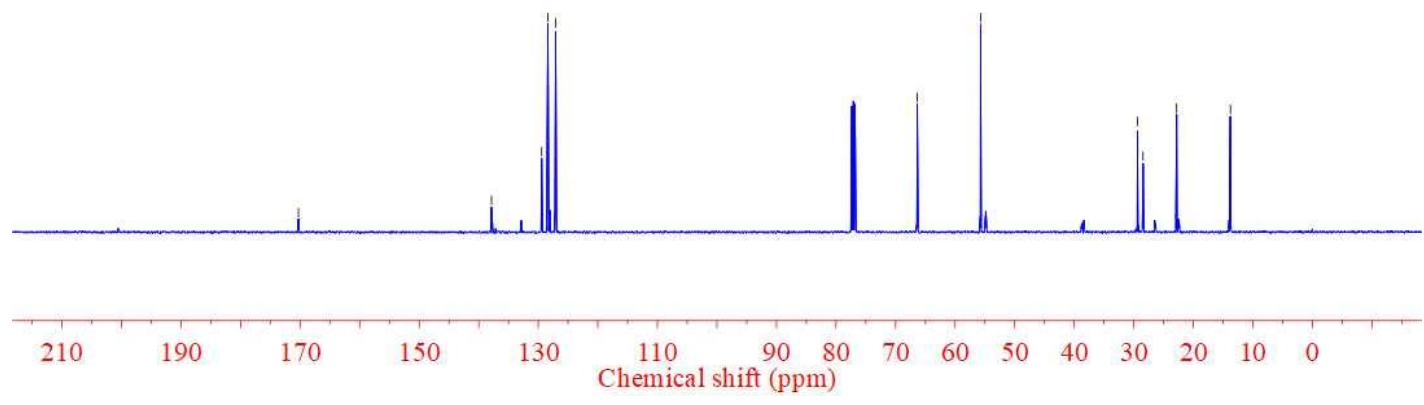

${ }^{13} \mathbf{C}\left\{{ }^{1} \mathbf{H}\right\}$ NMR Spectrum of Compound $1 u$ 
<smiles>c1ccc2c(c1)CCC/C2=N\N1CCOCC1</smiles>

${ }^{1} \mathrm{H}$ NMR $\left(\mathrm{CDCl}_{3}, 500 \mathrm{MHz}\right)$

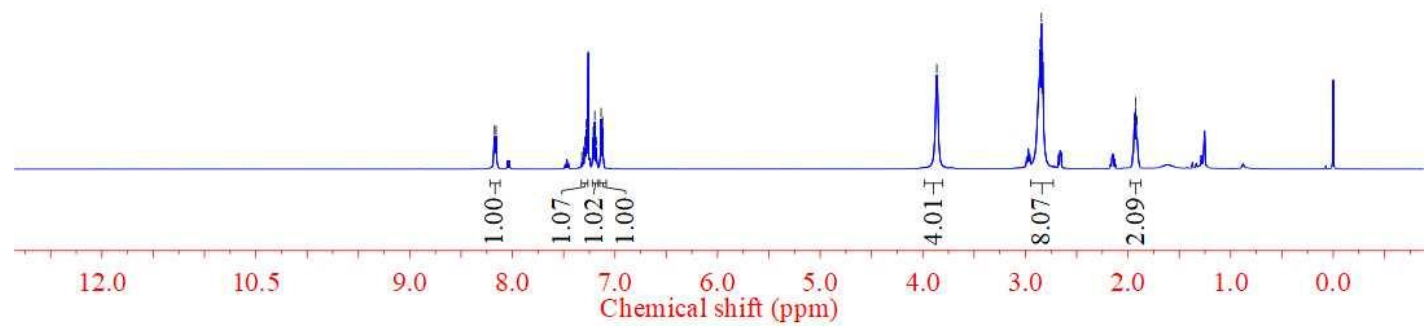

${ }^{1} \mathrm{H}$ NMR Spectrum of Compound $1 \mathrm{v}$

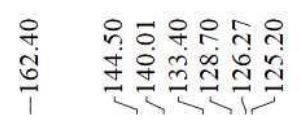<smiles>c1ccc2c(c1)CCC/C2=N\N1CCOCC1</smiles>

${ }^{13} \mathrm{C}\left\{{ }^{1} \mathrm{H}\right\}$ NMR $\left(\mathrm{CDCl}_{3}, 126 \mathrm{MHz}\right)$ 


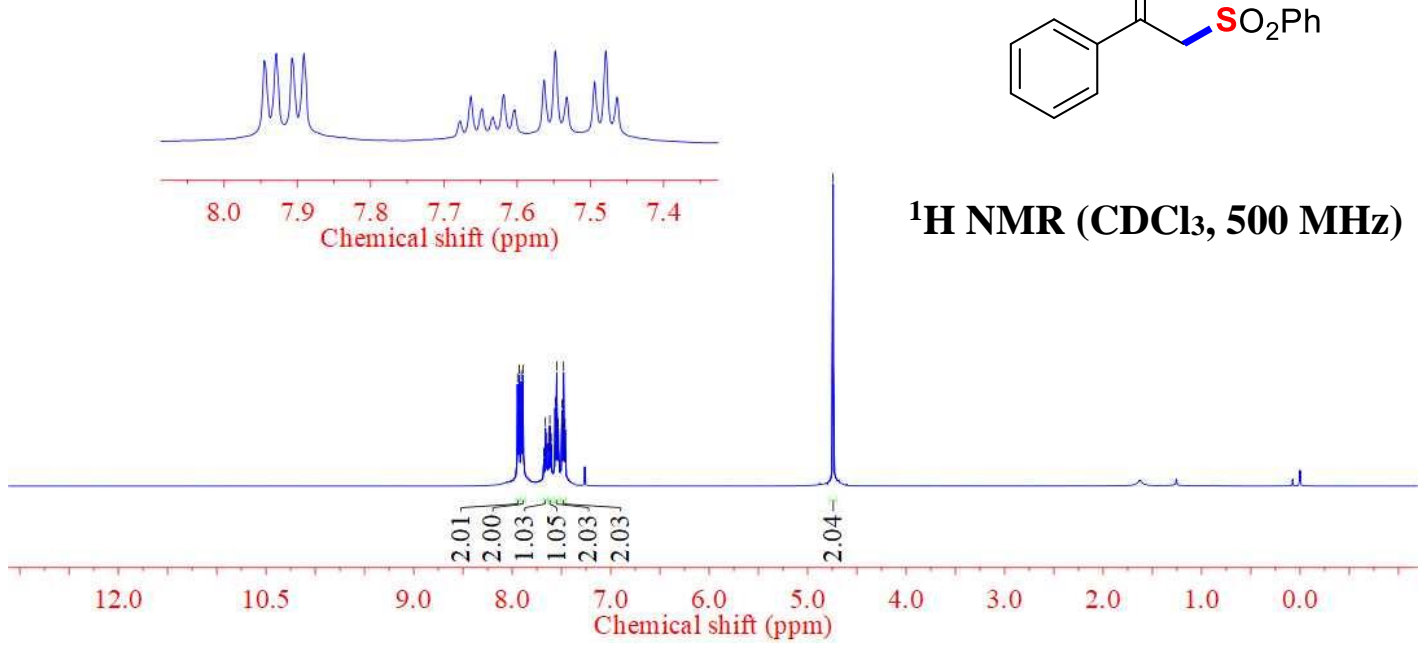

${ }^{1} \mathrm{H}$ NMR Spectrum of Compound 3a

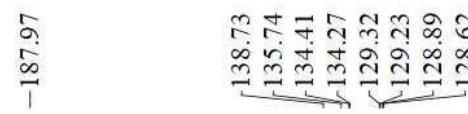

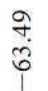
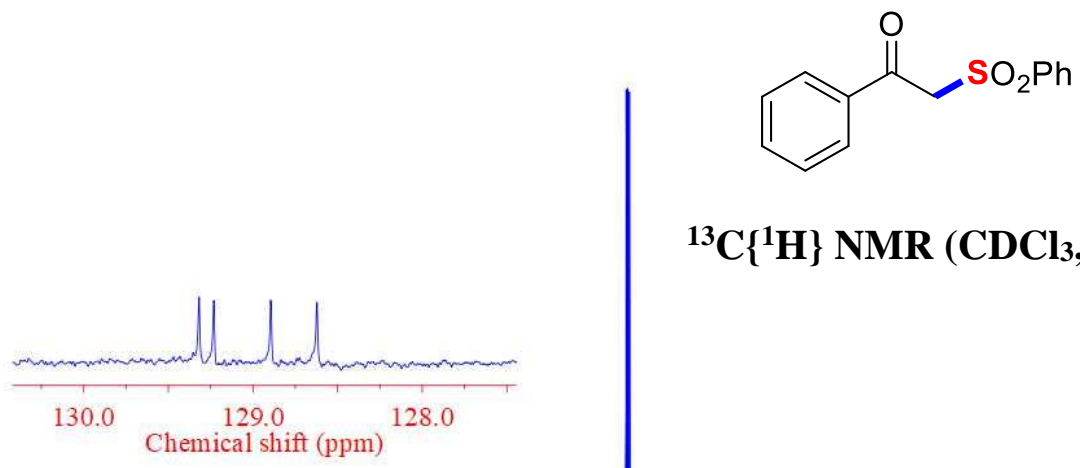

${ }^{13} \mathrm{C}\left\{{ }^{1} \mathrm{H}\right\}$ NMR $\left(\mathrm{CDCl}_{3}, 126 \mathrm{MHz}\right)$

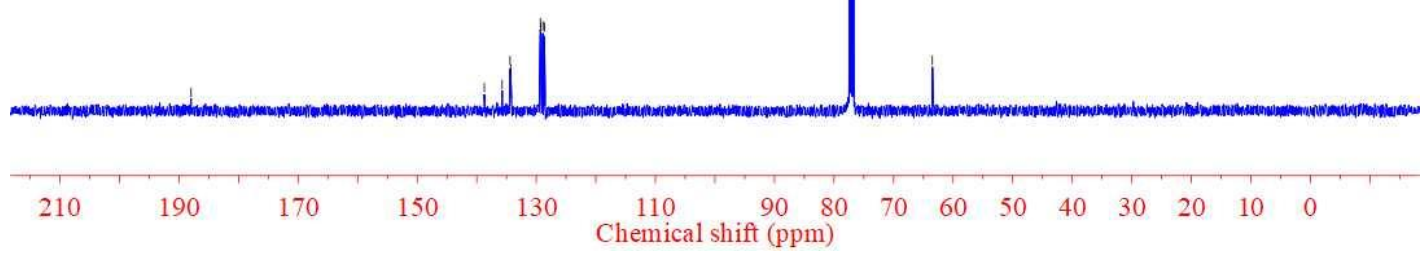

${ }^{13} \mathbf{C}\left\{{ }^{1} \mathbf{H}\right\}$ NMR Spectrum of Compound 3a 


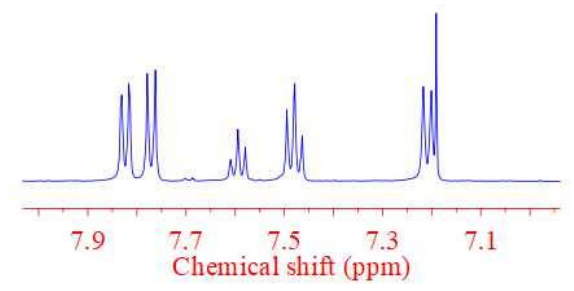<smiles>Cc1ccc(C(=O)CS(=O)(=O)c2ccccc2)cc1</smiles>

${ }^{1} \mathrm{H}$ NMR (CDCl3, $\left.500 \mathrm{MHz}\right)$

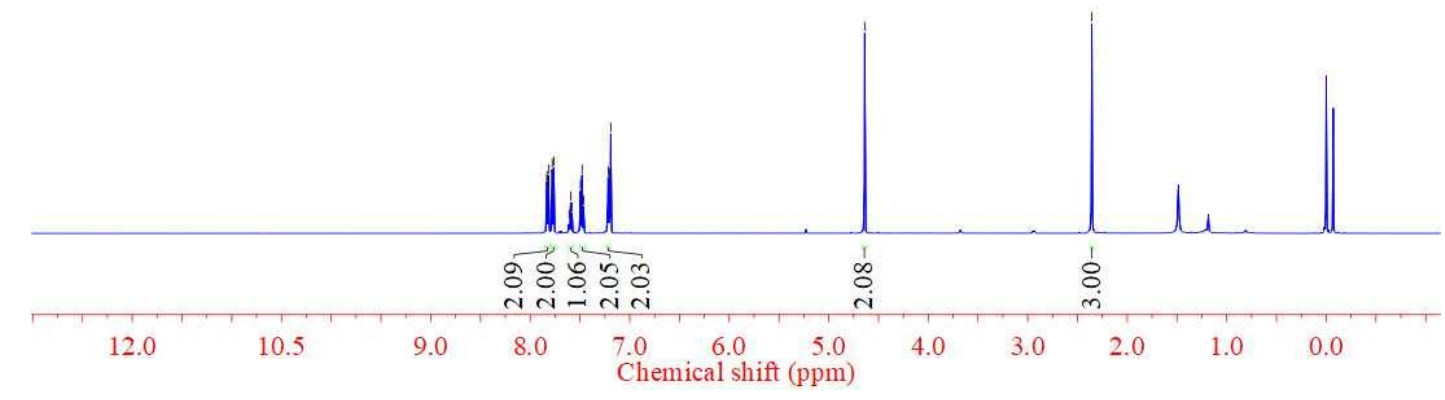

${ }^{1} \mathrm{H}$ NMR Spectrum of Compound $3 \mathrm{~b}$

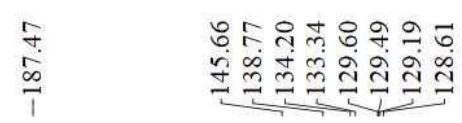

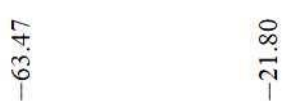

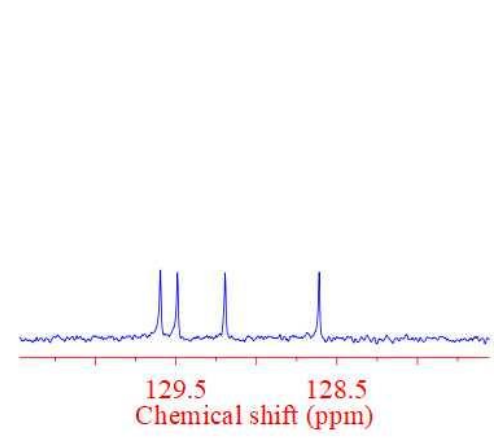<smiles>Cc1ccc(C(=O)CS(=O)(=O)c2ccccc2)cc1</smiles>

${ }^{13} \mathrm{C}\left\{{ }^{1} \mathrm{H}\right\}$ NMR $\left(\mathrm{CDCl}_{3}, 126 \mathrm{MHz}\right)$

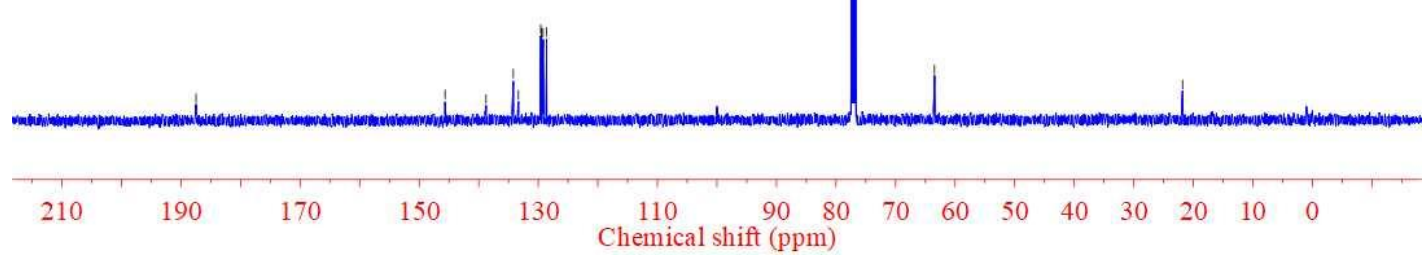

${ }^{13} \mathrm{C}\left\{{ }^{1} \mathrm{H}\right\}$ NMR Spectrum of Compound $3 \mathrm{~b}$ 

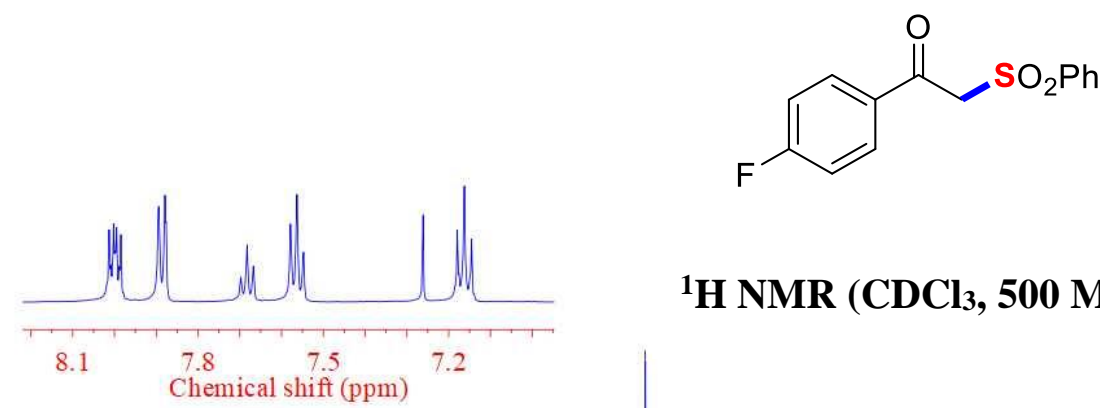

${ }^{1} \mathrm{H}$ NMR $\left(\mathrm{CDCl}_{3}, 500 \mathrm{MHz}\right)$

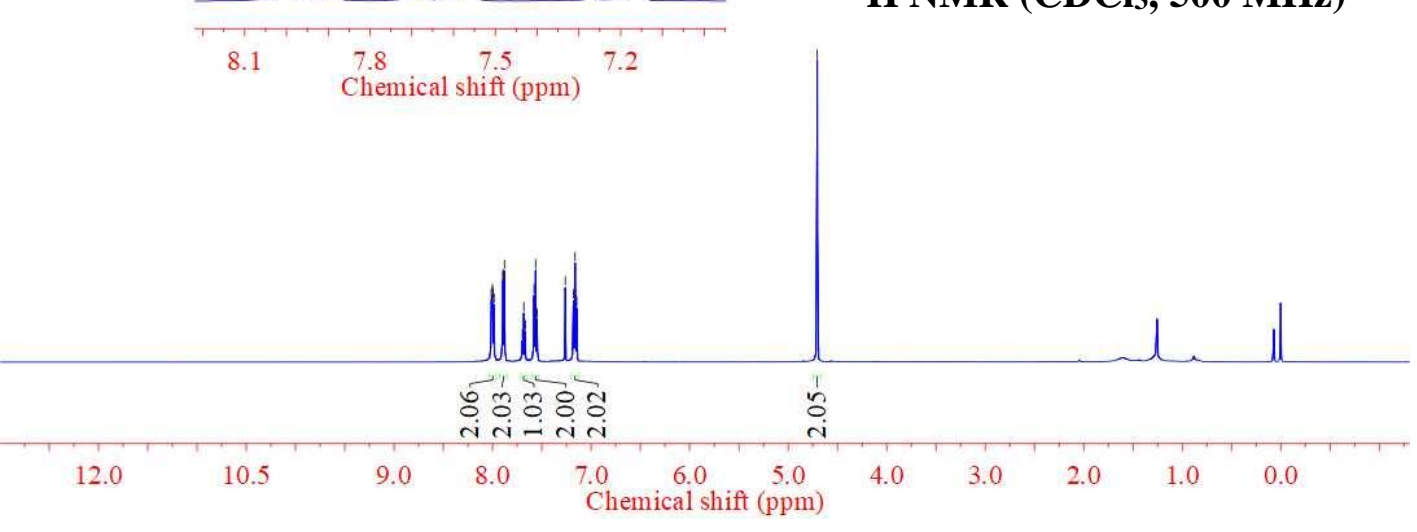

${ }^{1} \mathrm{H}$ NMR Spectrum of Compound 3c

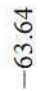<smiles>O=C(CS(=O)(=O)c1ccccc1)c1ccc(F)cc1</smiles>

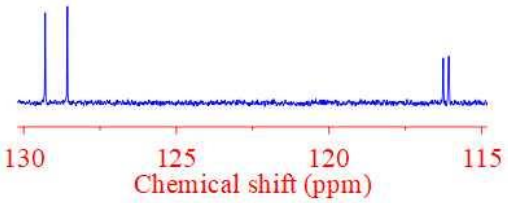

${ }^{13} \mathrm{C}\left\{{ }^{1} \mathrm{H}\right\} \mathrm{NMR}\left(\mathrm{CDCl}_{3}, 126 \mathrm{MHz}\right)$

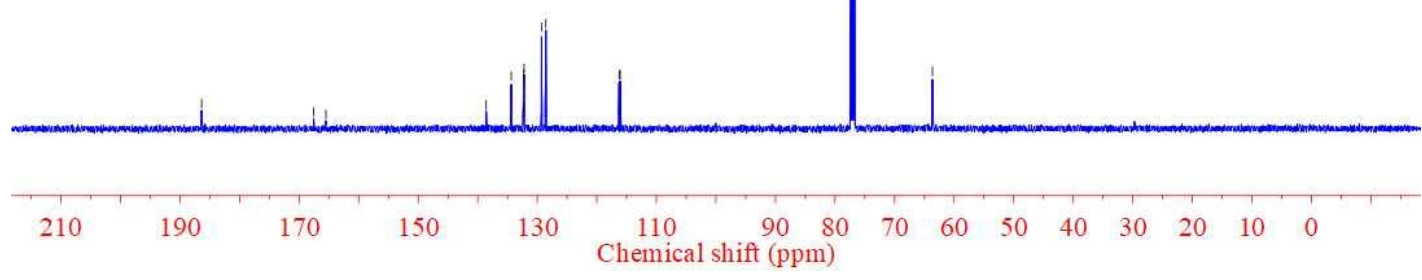

${ }^{13} \mathrm{C}\left\{{ }^{1} \mathrm{H}\right\}$ NMR Spectrum of Compound $3 \mathrm{c}$ 
<smiles>O=C(CSc1ccccc1)c1ccc(F)cc1</smiles>

${ }^{19} \mathrm{~F}\left\{{ }^{1} \mathrm{H}\right\} \operatorname{NMR}\left(\mathrm{CDCl}_{3}, 471 \mathrm{MHz}\right)$

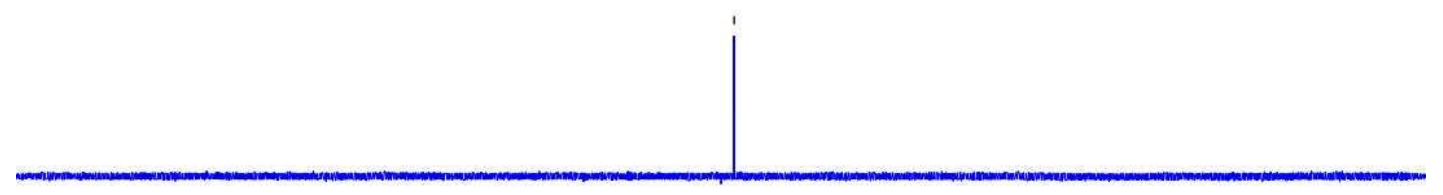

\begin{tabular}{|c|c|c|c|c|c|c|c|c|c|c|c|c|c|c|c|c|}
\hline 10 & 0 & -10 & -20 & -3 & - & 0 & -50 & -60 & -70 & $\begin{array}{l}-80-90 \\
\text { Chemical shift }\end{array}$ & $\begin{array}{l}-110 \\
(\mathrm{ppm})\end{array}$ & -130 & -150 & -170 & -190 & -210 \\
\hline
\end{tabular}

${ }^{19} \mathbf{F}\left\{{ }^{1} \mathbf{H}\right\}$ NMR Spectrum of Compound $3 \mathrm{c}$

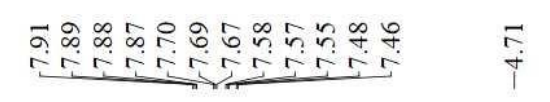
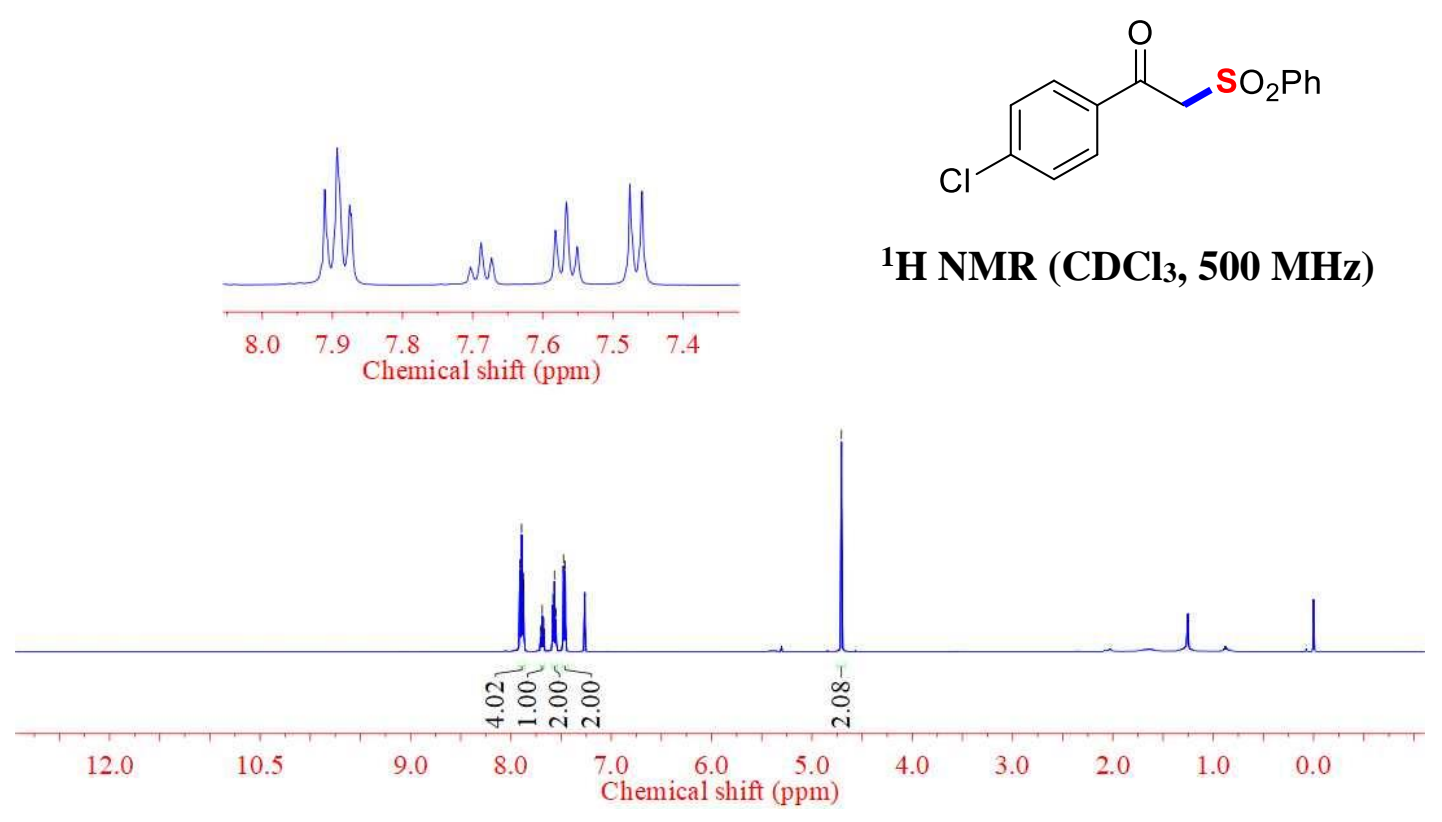

${ }^{1}$ H NMR Spectrum of Compound 3d 
<smiles>O=C(CS(=O)(=O)c1ccccc1)c1ccc(Cl)cc1</smiles>

${ }^{13} \mathrm{C}\left\{{ }^{1} \mathrm{H}\right\}$ NMR $\left(\mathrm{CDCl}_{3}, 126 \mathrm{MHz}\right)$
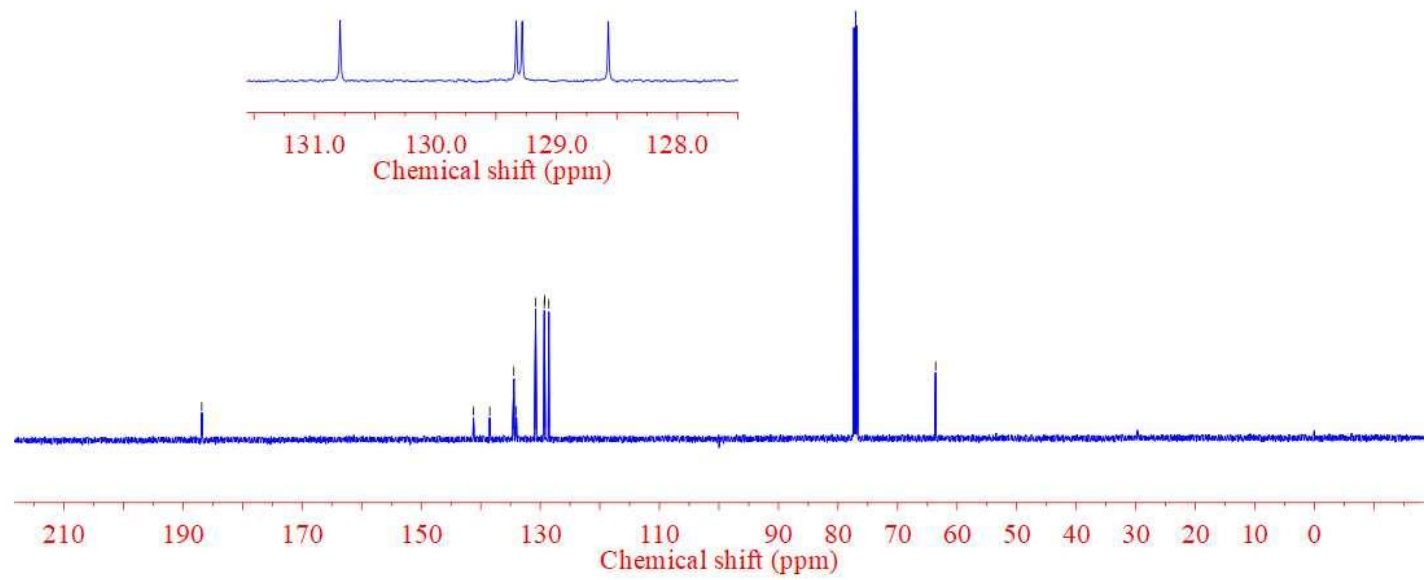

${ }^{13} \mathbf{C}\left\{{ }^{1} \mathbf{H}\right\}$ NMR Spectrum of Compound 3d

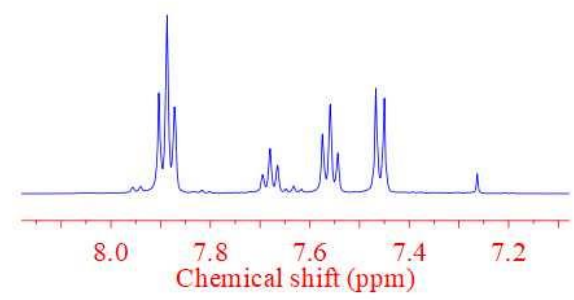<smiles>O=C(CS(=O)(=O)c1ccccc1)c1ccc(Br)cc1</smiles>

${ }^{1} \mathrm{H}$ NMR $\left(\mathrm{CDCl}_{3}, 500 \mathrm{MHz}\right)$

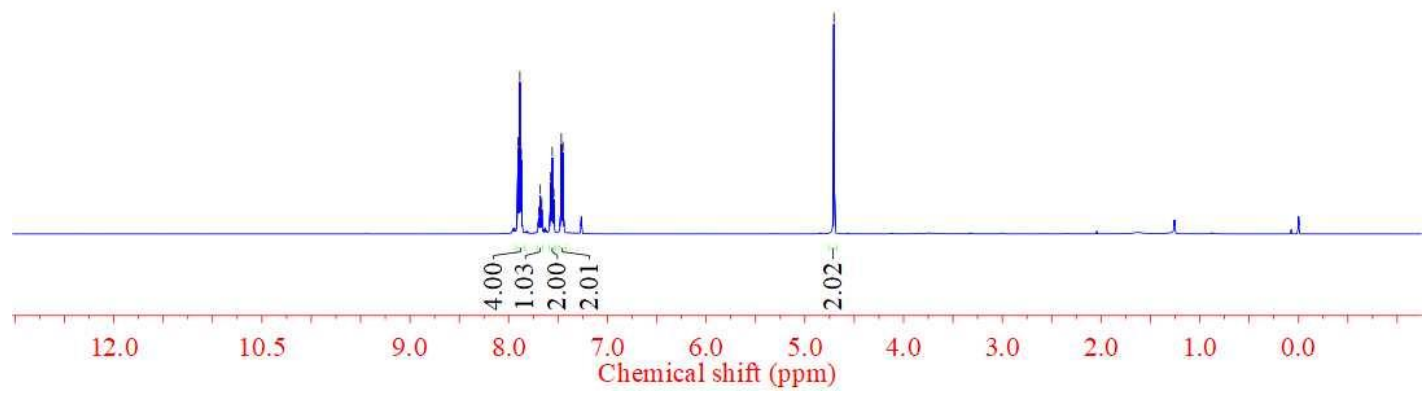

${ }^{1} \mathrm{H}$ NMR Spectrum of Compound $3 \mathrm{e}$ 
<smiles>O=C(CS(=O)(=O)c1ccccc1)c1ccc(Br)cc1</smiles>

${ }^{13} \mathrm{C}\left\{{ }^{1} \mathrm{H}\right\}$ NMR $\left(\mathrm{CDCl}_{3}, 126 \mathrm{MHz}\right)$
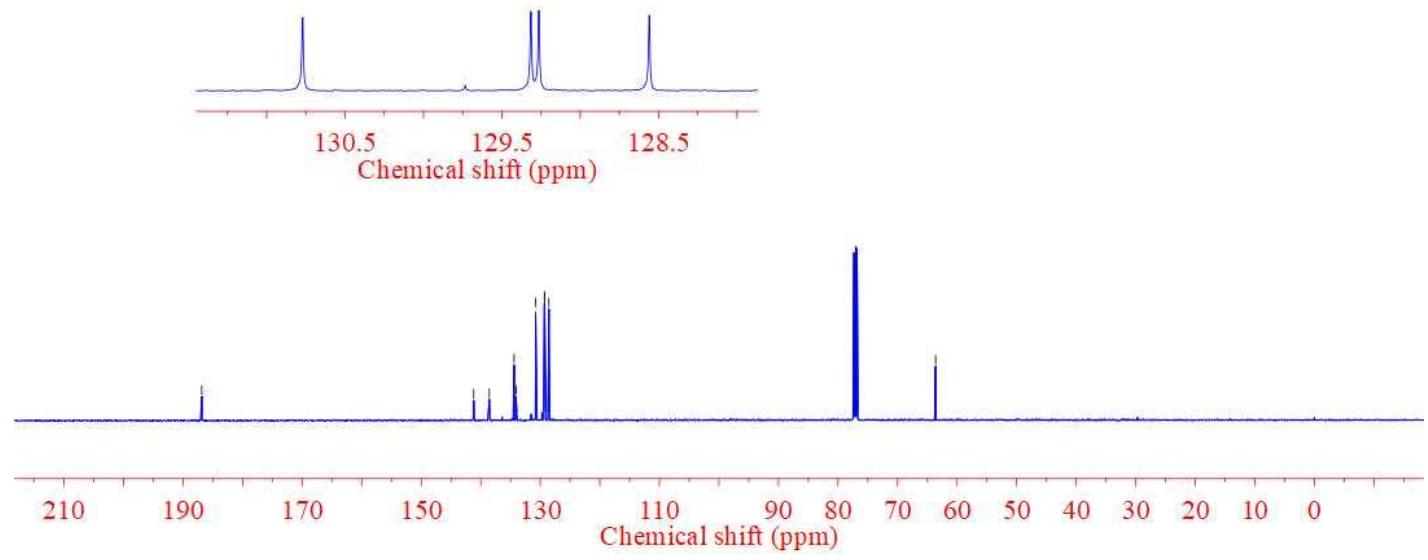

${ }^{13} \mathbf{C}\left\{{ }^{1} \mathrm{H}\right\}$ NMR Spectrum of Compound $3 \mathrm{e}$

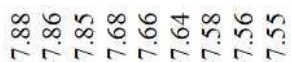

$\stackrel{?}{\stackrel{+}{+}}$<smiles>O=C(CS(=O)(=O)c1ccccc1)c1ccc(I)cc1</smiles>

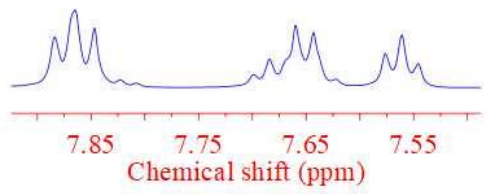

${ }^{1} \mathrm{H}$ NMR $\left(\mathrm{CDCl}_{3}, 500 \mathrm{MHz}\right)$

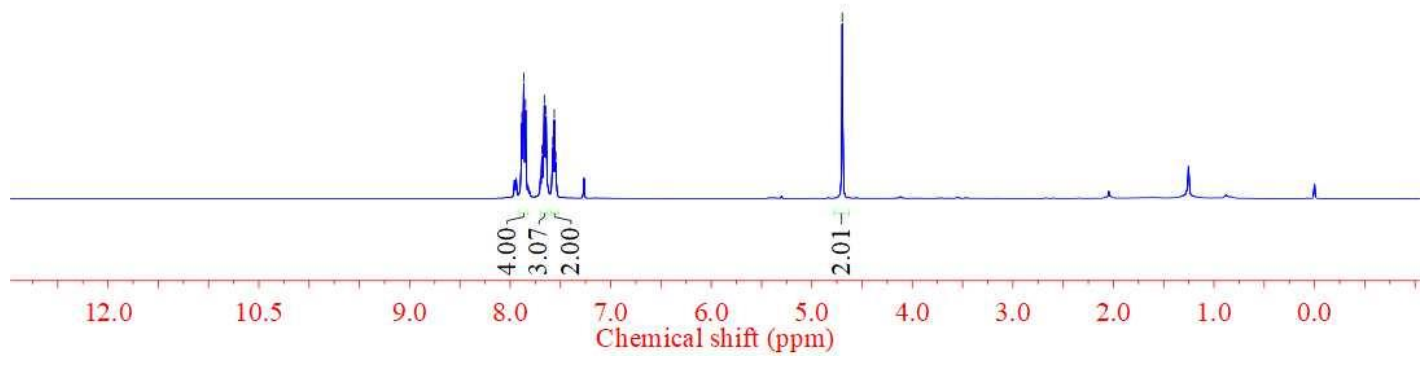

${ }^{1} \mathrm{H}$ NMR Spectrum of Compound $3 f$ 
<smiles>O=C(CS(=O)(=O)c1ccccc1)c1ccc(I)cc1</smiles>

${ }^{13} \mathrm{C}\left\{{ }^{1} \mathrm{H}\right\}$ NMR $\left(\mathrm{CDCl}_{3}, 126 \mathrm{MHz}\right)$
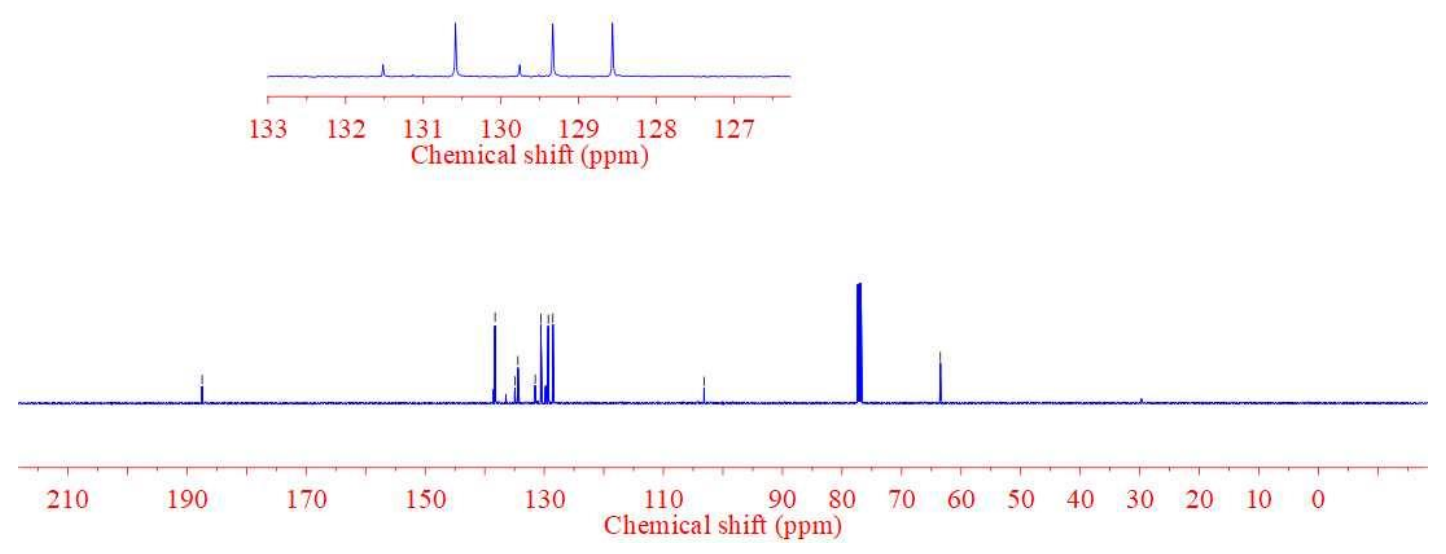

${ }^{13} \mathrm{C}\left\{{ }^{1} \mathrm{H}\right\}$ NMR Spectrum of Compound $3 f$

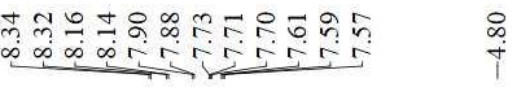
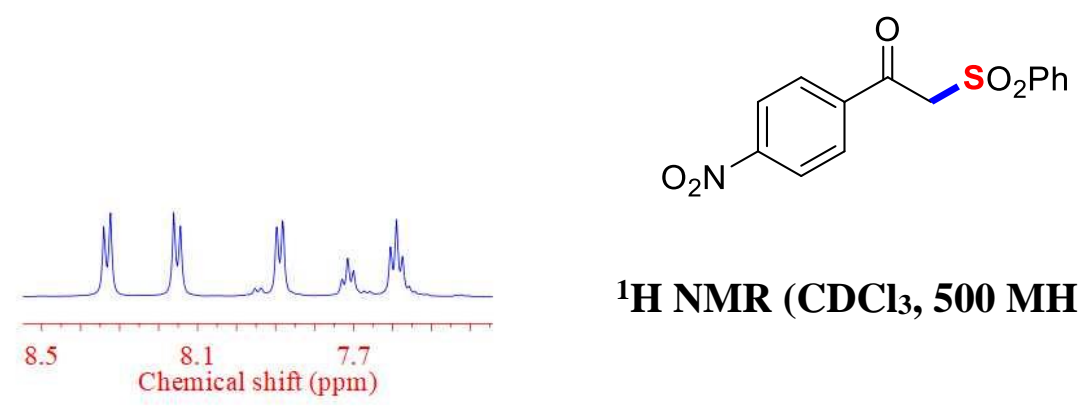

${ }^{1} \mathrm{H} \mathrm{NMR}\left(\mathrm{CDCl}_{3}, 500 \mathrm{MHz}\right)$

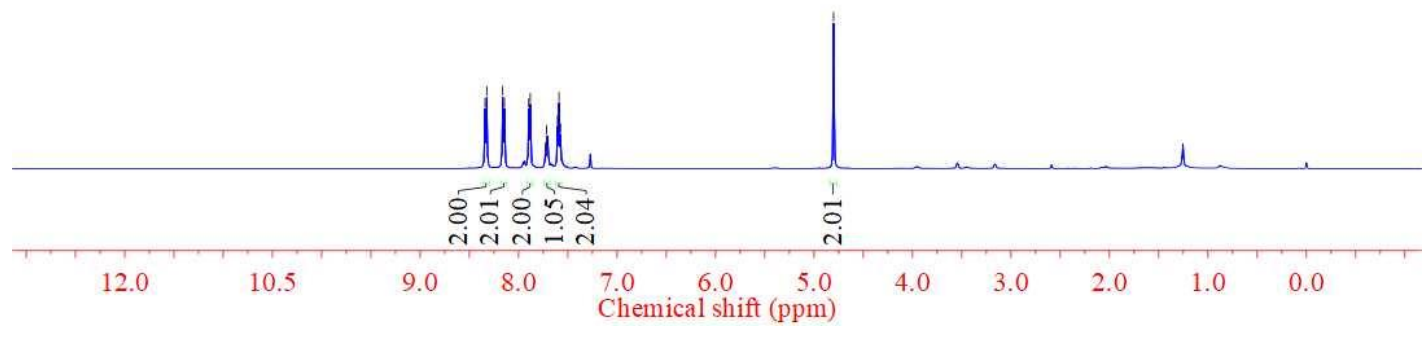

${ }^{1} \mathrm{H}$ NMR Spectrum of Compound $3 \mathrm{~g}$ 


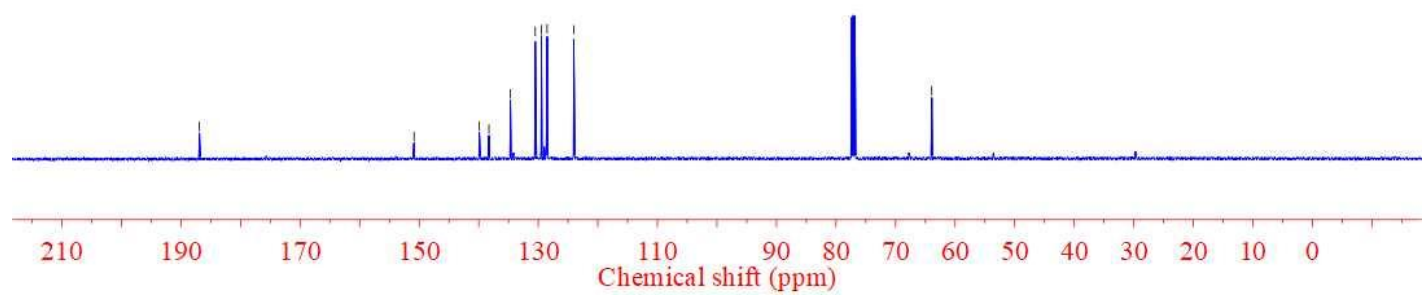

${ }^{13} \mathbf{C}\left\{{ }^{1} \mathbf{H}\right\}$ NMR Spectrum of Compound $3 g$

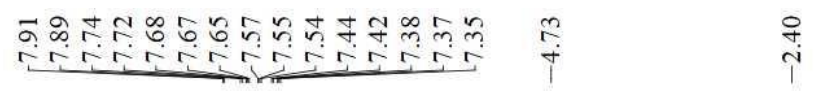<smiles>Cc1cccc(C(=O)CS(=O)(=O)c2ccccc2)c1</smiles>

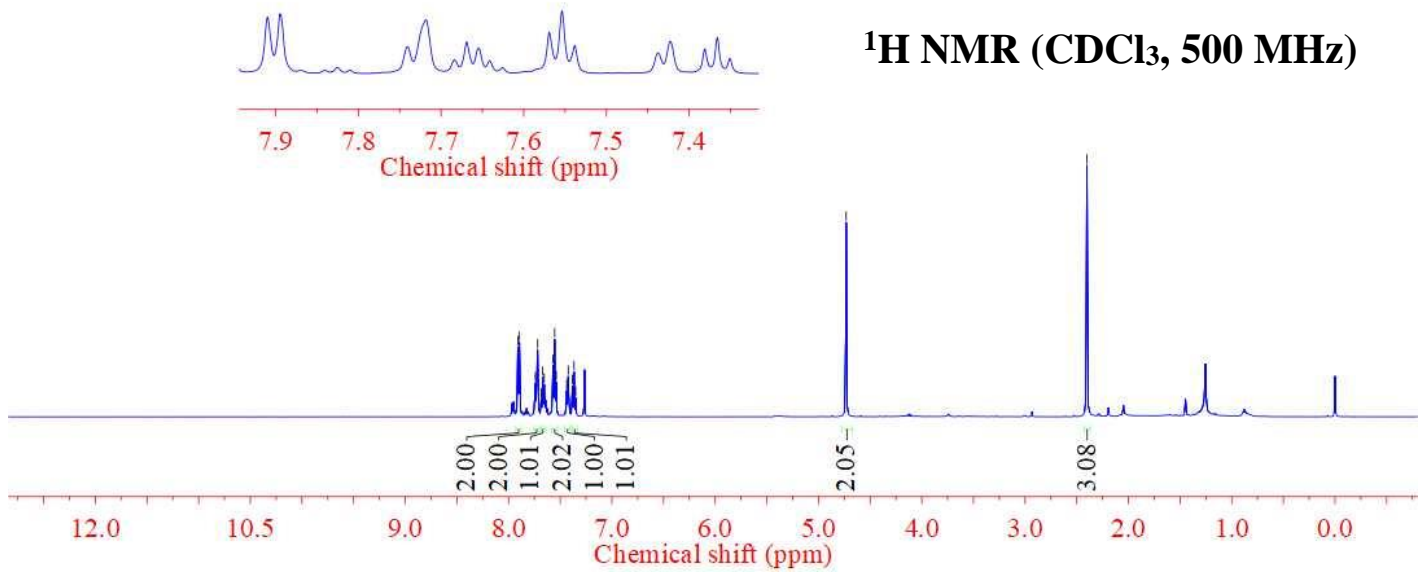

${ }^{1} \mathrm{H}$ NMR $\left(\mathrm{CDCl}_{3}, 500 \mathrm{MHz}\right)$

${ }^{1} \mathrm{H}$ NMR Spectrum of Compound $3 \mathrm{~h}$ 
<smiles>Cc1cccc(C(=O)CS(=O)(=O)c2ccccc2)c1</smiles>

${ }^{13} \mathrm{C}\left\{{ }^{1} \mathrm{H}\right\}$ NMR $\left(\mathrm{CDCl}_{3}, 126 \mathrm{MHz}\right)$
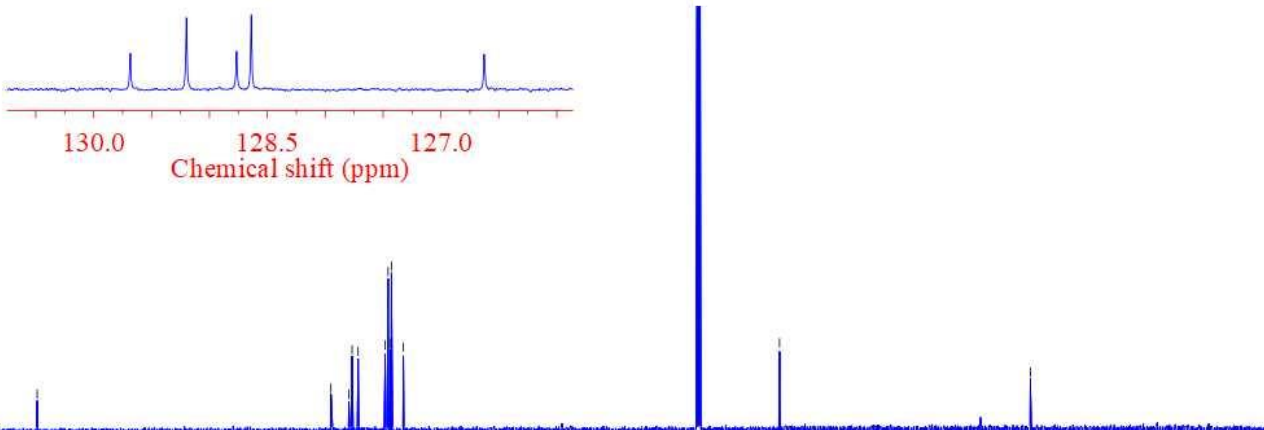

$210 \quad 190$

$170 \quad 150 \quad 130 \quad \begin{gathered}110 \\ \text { Chemical shift (ppm) }\end{gathered}$

${ }^{13} \mathrm{C}\left\{{ }^{1} \mathrm{H}\right\}$ NMR Spectrum of Compound $3 \mathrm{~h}$

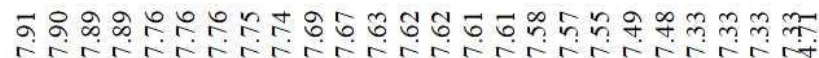

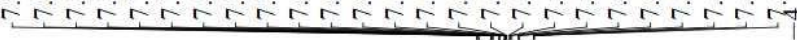

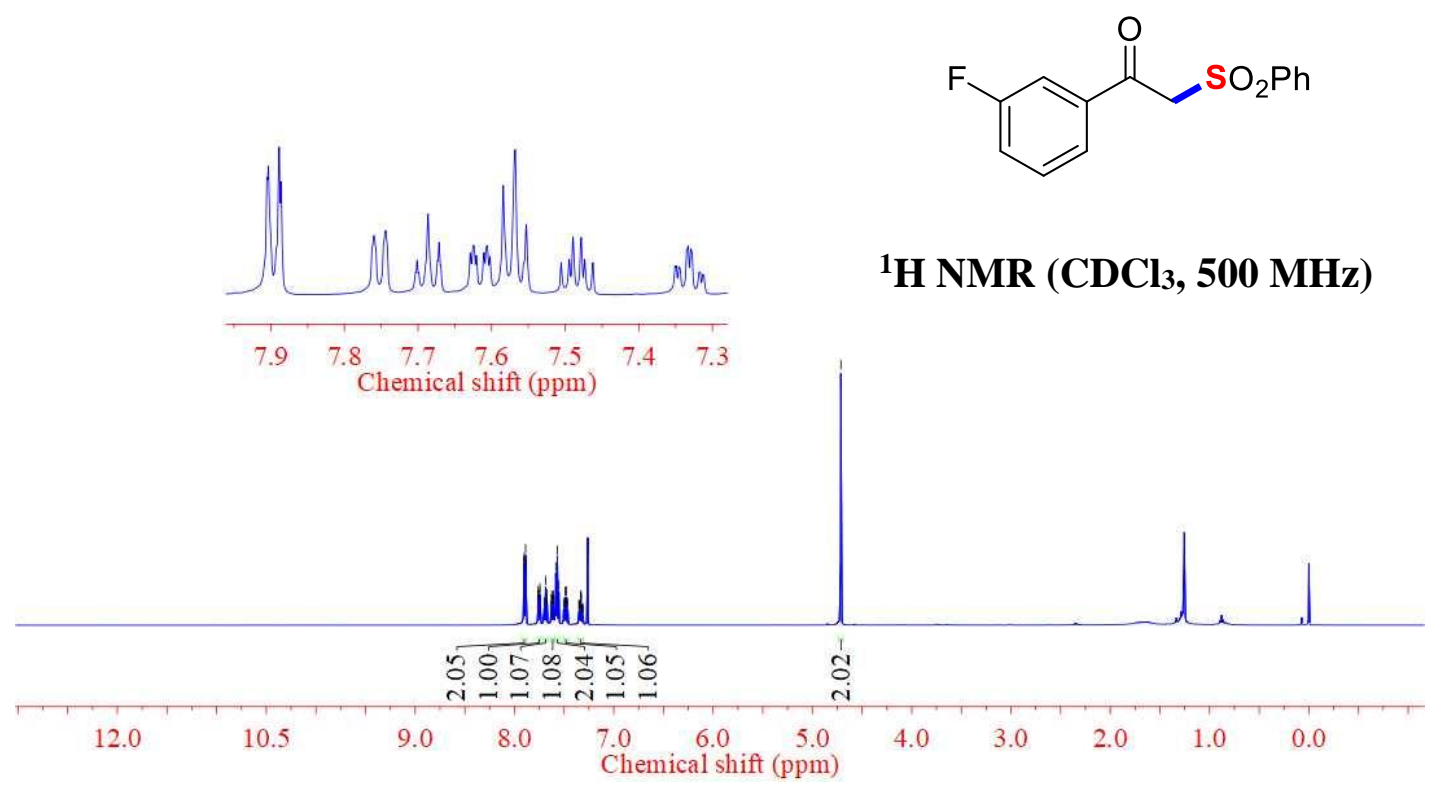

${ }^{1}$ H NMR Spectrum of Compound 3i 
<smiles>O=C(CS(=O)(=O)c1ccccc1)c1cccc(F)c1</smiles>

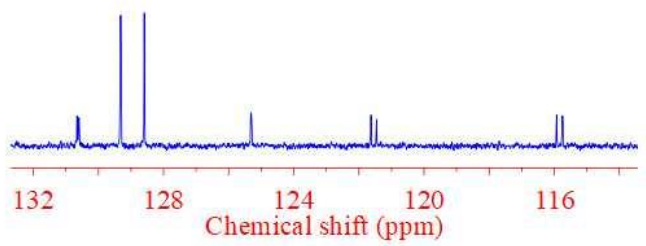

${ }^{13} \mathrm{C}\left\{{ }^{1} \mathrm{H}\right\} \mathrm{NMR}\left(\mathrm{CDCl}_{3}, 126 \mathrm{MHz}\right)$

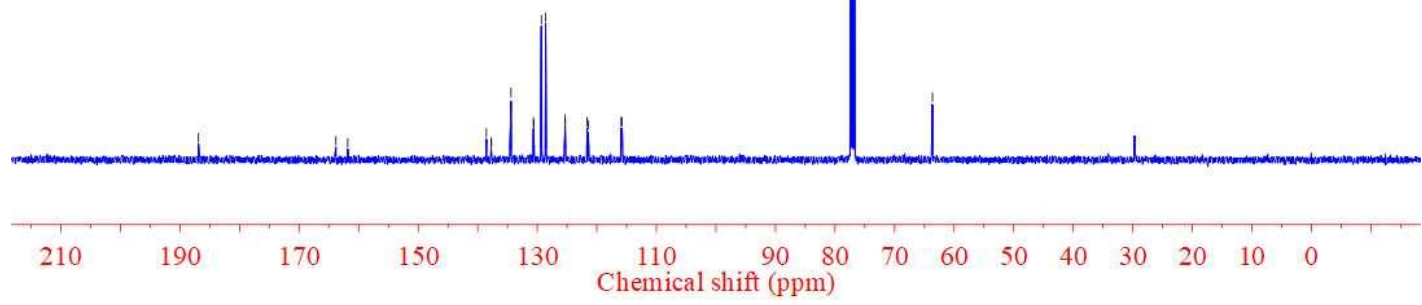

${ }^{13} \mathbf{C}\left\{{ }^{1} \mathrm{H}\right\}$ NMR Spectrum of Compound 3i

$\stackrel{8}{\circ}$<smiles>O=C(CS(=O)(=O)c1ccccc1)c1cccc(F)c1</smiles>

${ }^{19} \mathrm{~F}\left\{{ }^{1} \mathrm{H}\right\}$ NMR $\left(\mathrm{CDCl}_{3}, 471 \mathrm{MHz}\right)$

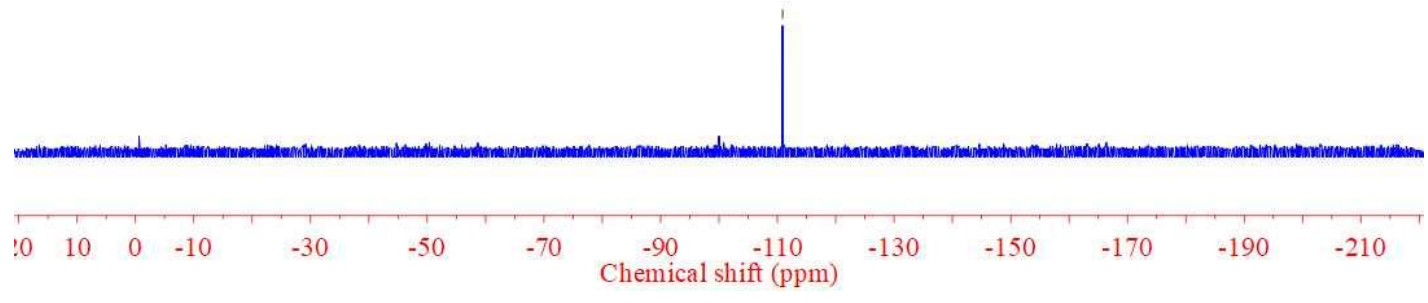

${ }^{19} \mathbf{F}\left\{{ }^{1} \mathbf{H}\right\}$ NMR Spectrum of Compound 3i 


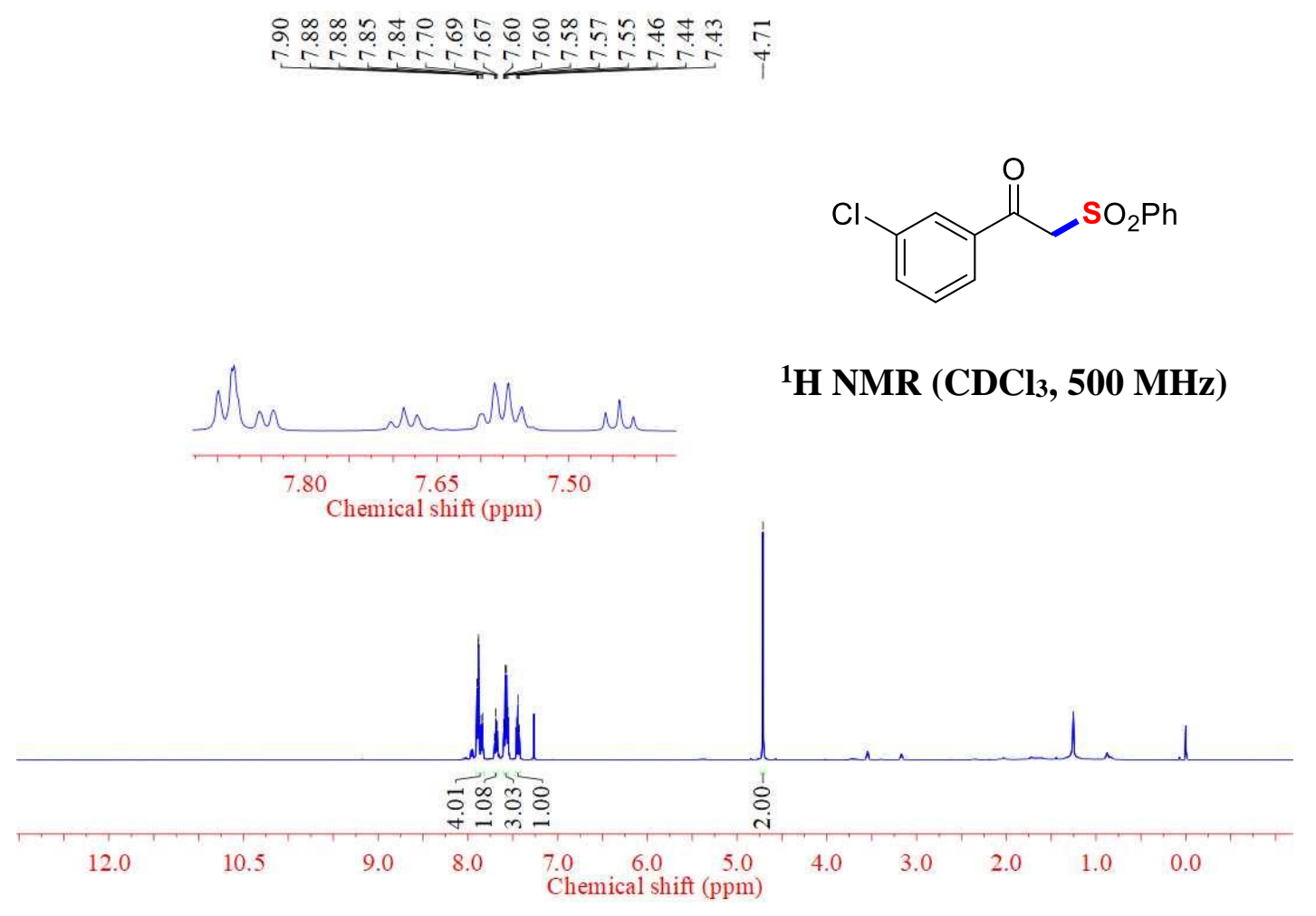

${ }^{1}$ H NMR Spectrum of Compound 3j
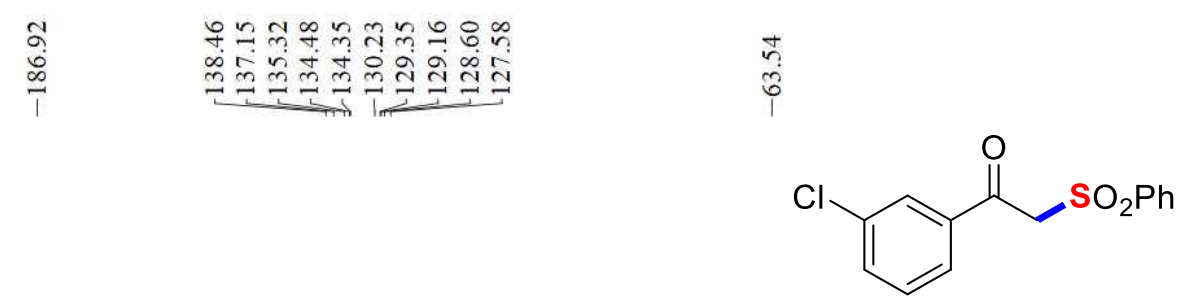

${ }^{13} \mathrm{C}\left\{{ }^{1} \mathrm{H}\right\}$ NMR $\left(\mathrm{CDCl}_{3}, 126 \mathrm{MHz}\right)$
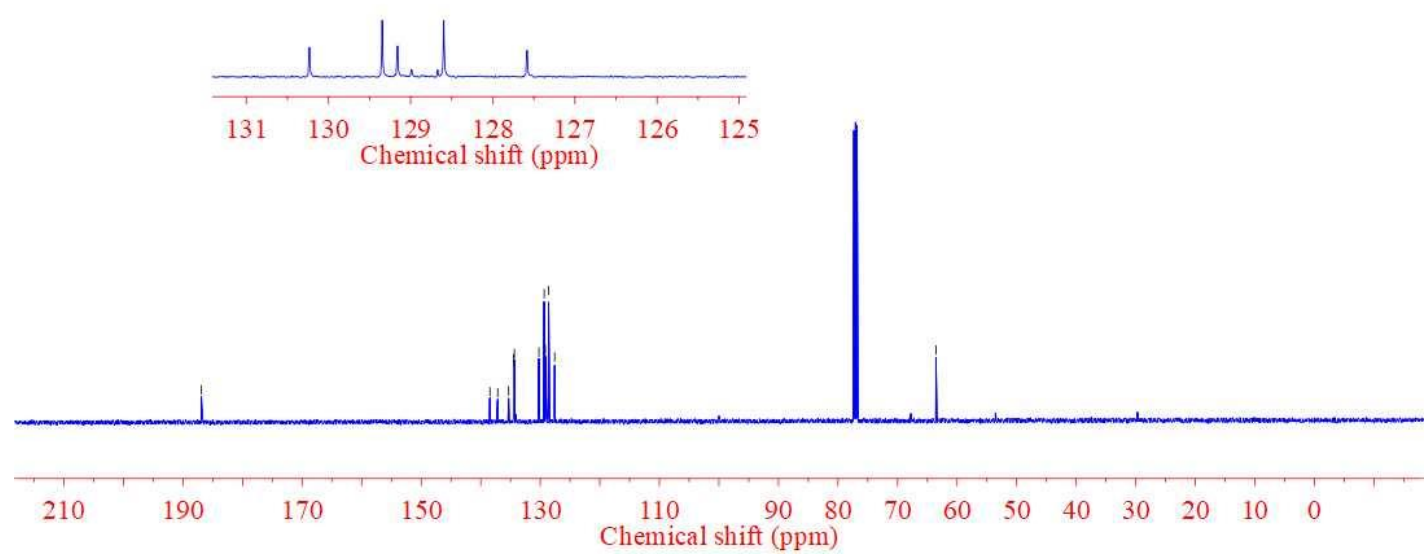

${ }^{13} \mathbf{C}\left\{{ }^{1} \mathbf{H}\right\}$ NMR Spectrum of Compound 3j 


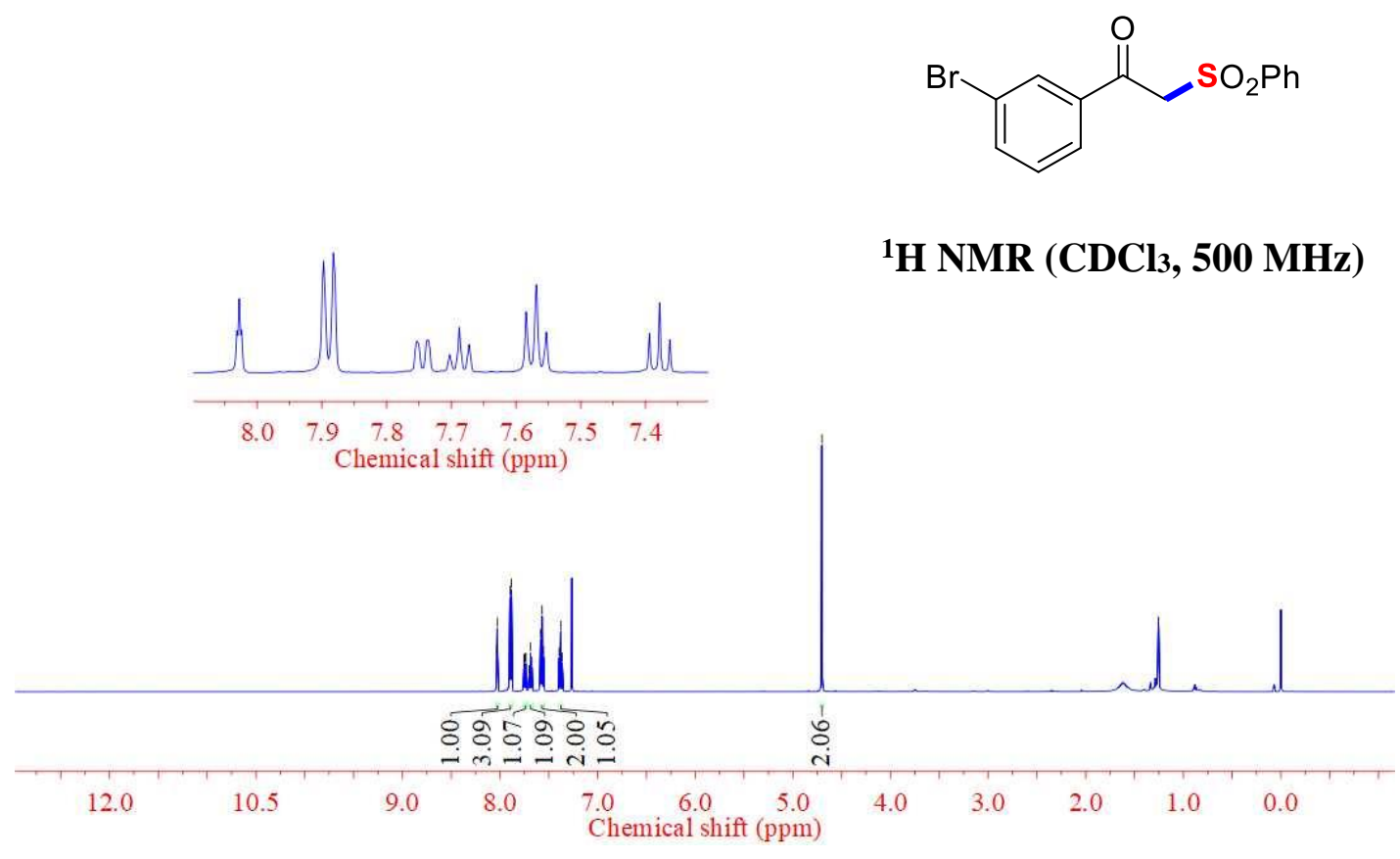

${ }^{1} \mathrm{H}$ NMR Spectrum of Compound 3k

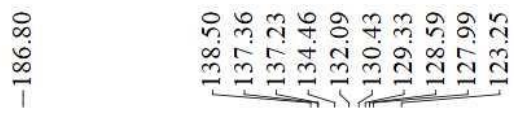

ñ<smiles>O=C(CS(=O)(=O)c1ccccc1)c1cccc(Br)c1</smiles>

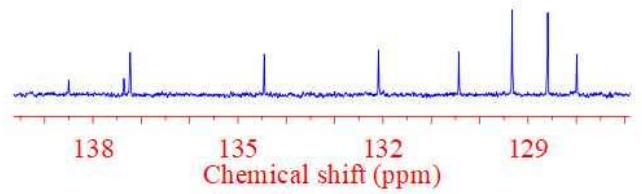

${ }^{13} \mathrm{C}\left\{{ }^{1} \mathrm{H}\right\}$ NMR $\left(\mathrm{CDCl}_{3}, 126 \mathrm{MHz}\right)$

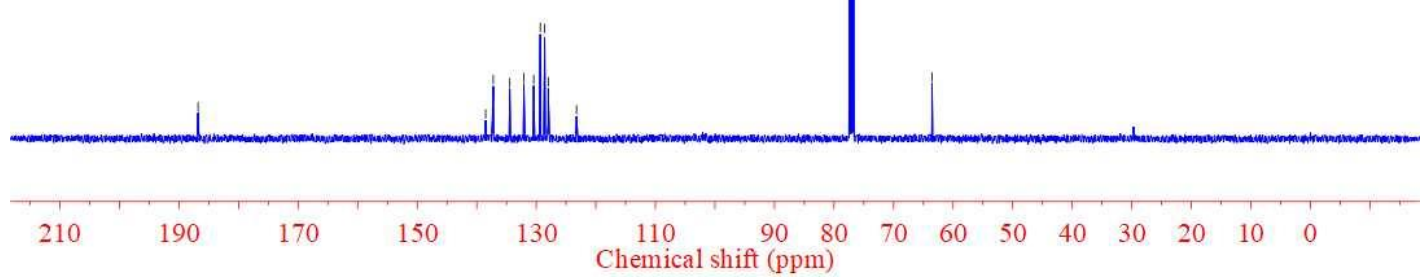

${ }^{13} \mathrm{C}\left\{{ }^{1} \mathrm{H}\right\}$ NMR Spectrum of Compound 3k 
<smiles>O=C(CS(=O)(=O)c1ccccc1)c1cccc(C(F)(F)F)c1</smiles>

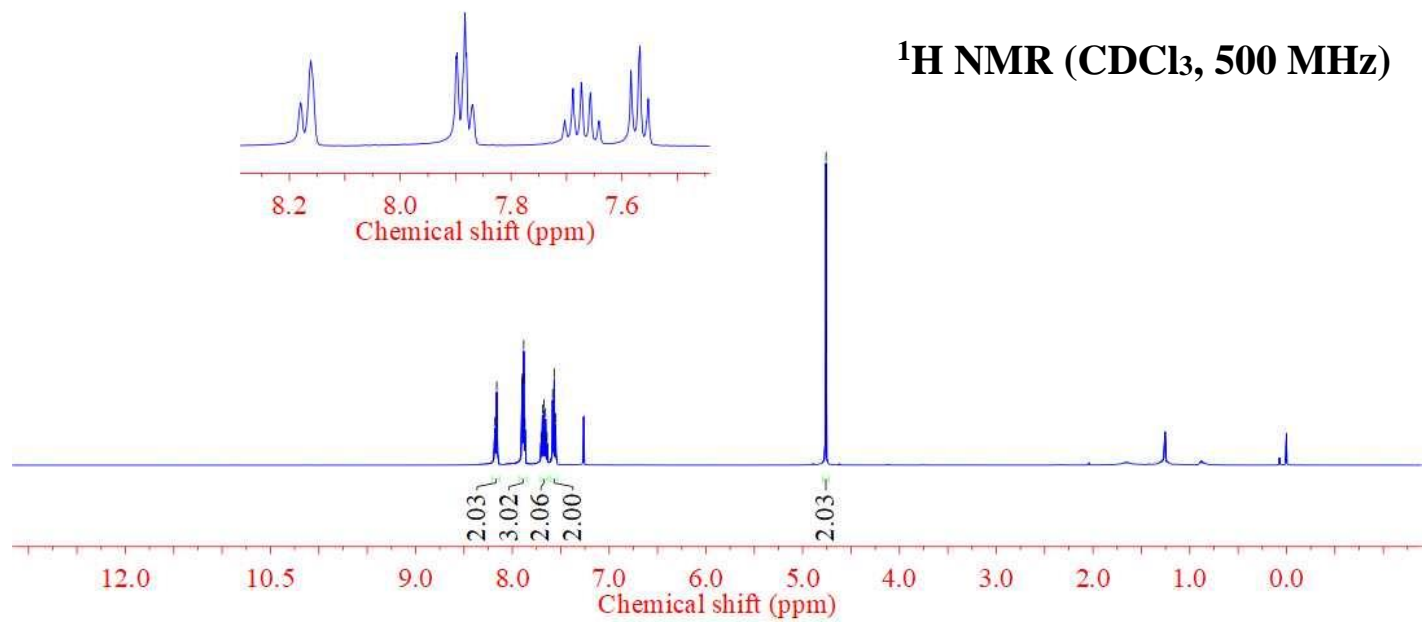

${ }^{1} \mathrm{H}$ NMR $\left(\mathrm{CDCl}_{3}, 500 \mathrm{MHz}\right)$

${ }^{1}$ H NMR Spectrum of Compound 31<smiles>O=C(CS(=O)(=O)c1ccccc1)c1cccc(C(F)(F)F)c1</smiles>

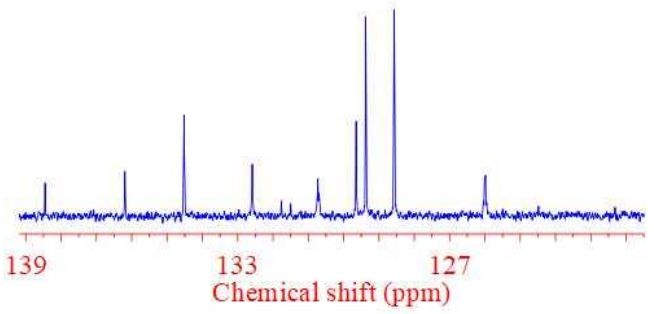

${ }^{13} \mathrm{C}\left\{{ }^{1} \mathrm{H}\right\}$ NMR $\left(\mathrm{CDCl}_{3}, 126 \mathrm{MHz}\right)$

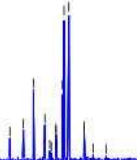

210

190

150

130

110

$90 \quad 80$

${ }^{13} \mathrm{C}\left\{{ }^{1} \mathrm{H}\right\}$ NMR Spectrum of Compound 31 
<smiles>O=C(CS(=O)(=O)c1ccccc1)c1cccc(C(F)(F)F)c1</smiles>

${ }^{19} \mathrm{~F}\left\{{ }^{1} \mathrm{H}\right\} \mathrm{NMR}\left(\mathrm{CDCl}_{3}, 471 \mathrm{MHz}\right)$

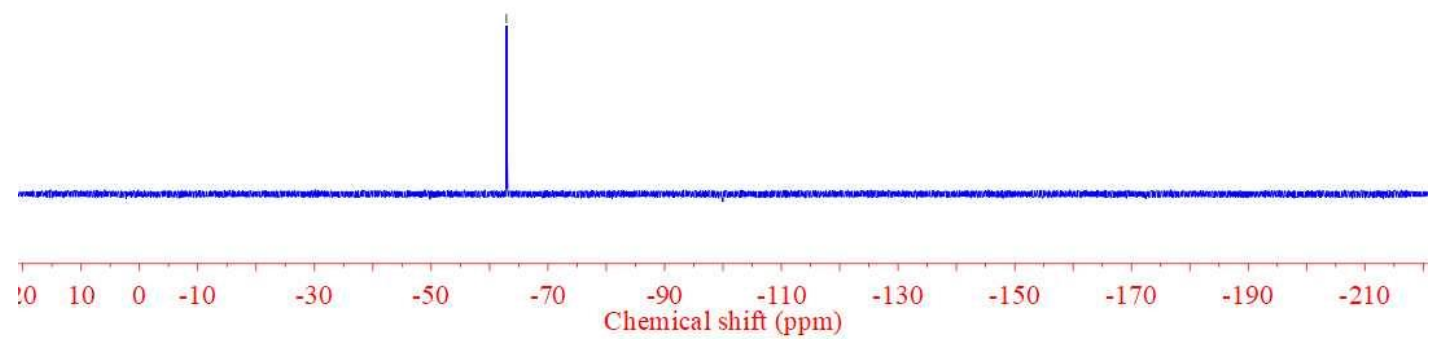

\section{${ }^{19} \mathbf{F}\left\{{ }^{1} \mathrm{H}\right\}$ NMR Spectrum of Compound 31}

\section{Mass Spectrum SmartFormula Report}

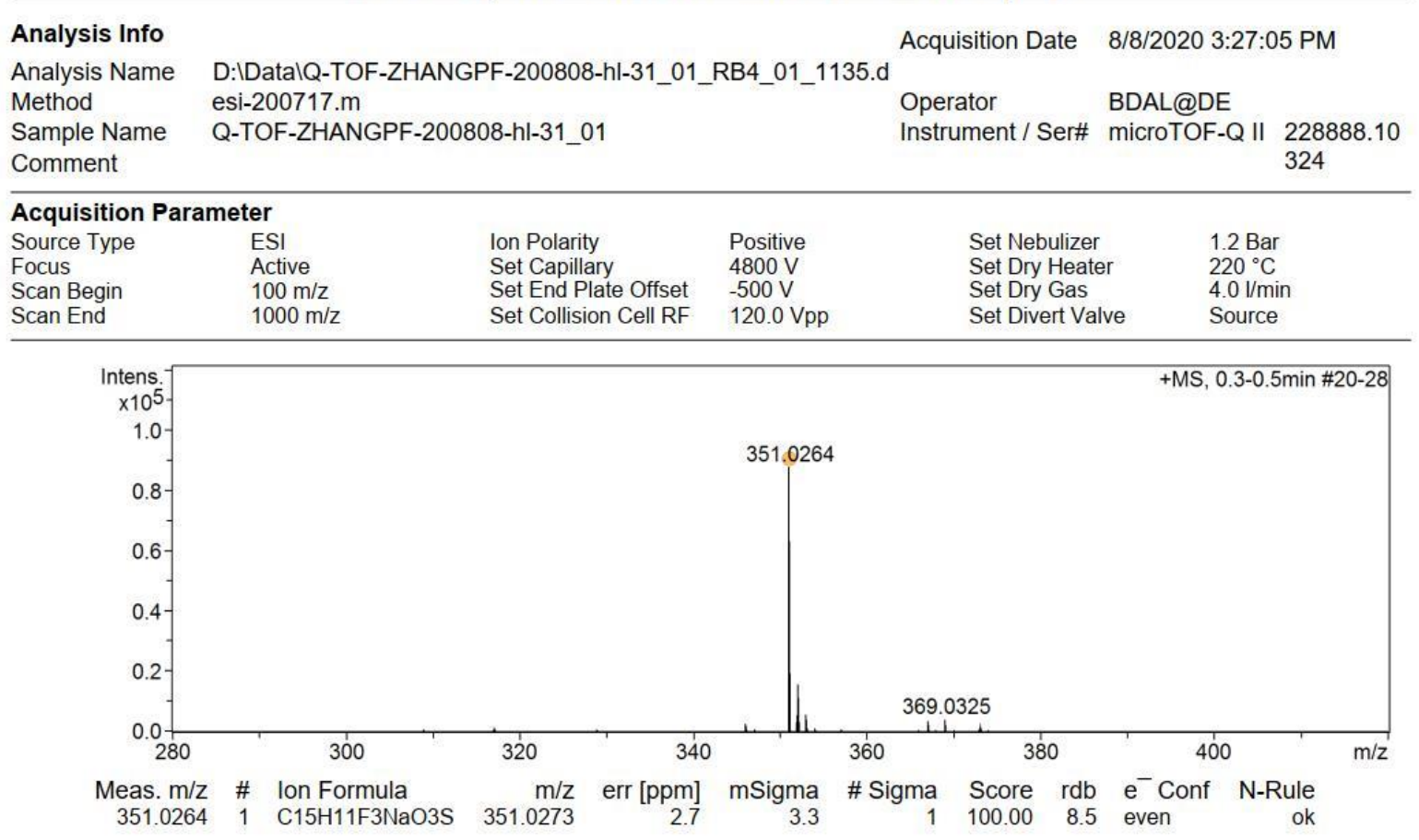

HRMS Spectrum of Compound 31 
<smiles>Cc1ccccc1C(=O)CS(=O)(=O)c1ccccc1</smiles>

${ }^{1} \mathrm{H}$ NMR $\left(\mathrm{CDCl}_{3}, 500 \mathrm{MHz}\right)$

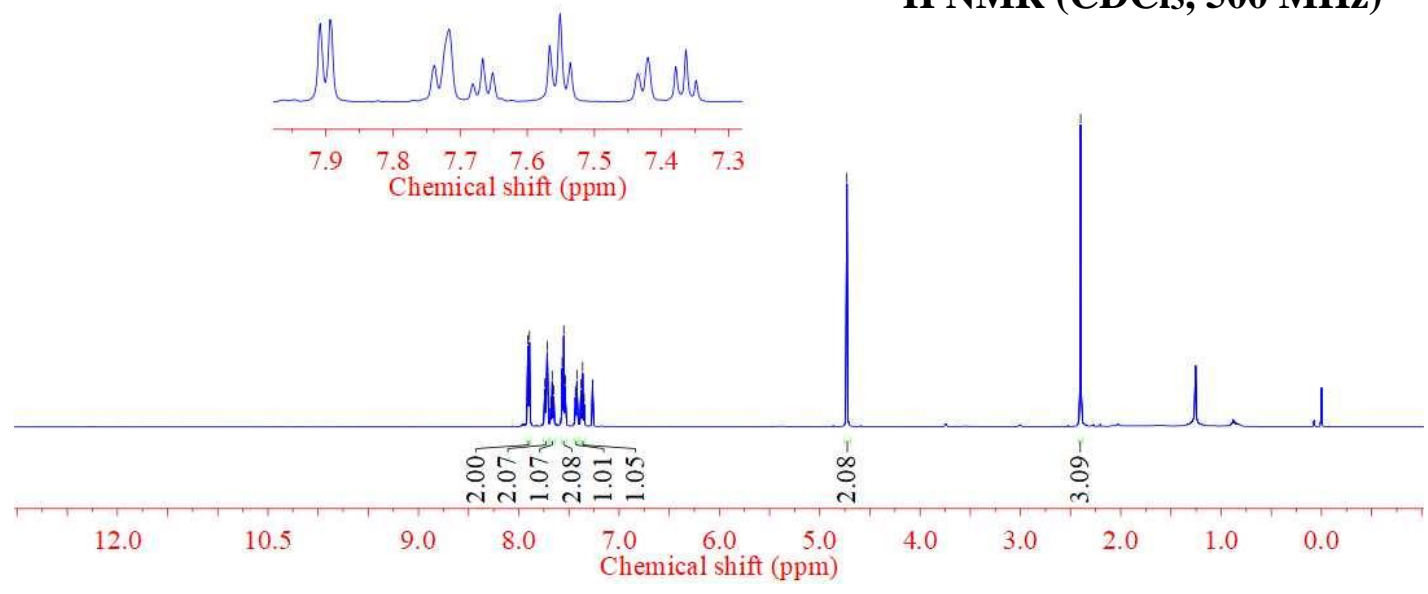

${ }^{1} \mathrm{H}$ NMR Spectrum of Compound 3m

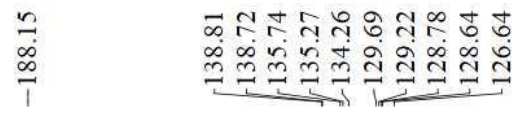

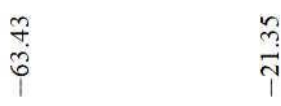<smiles>Cc1ccccc1C(=O)CSc1ccccc1</smiles>

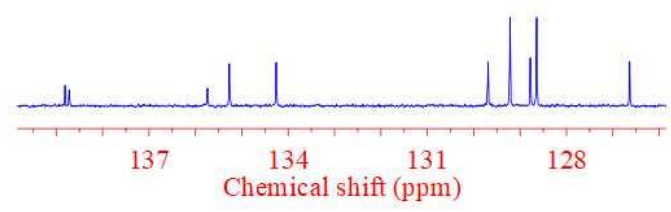

${ }^{13} \mathrm{C}\left\{{ }^{1} \mathrm{H}\right\} \mathrm{NMR}\left(\mathrm{CDCl}_{3}, 126 \mathrm{MHz}\right)$

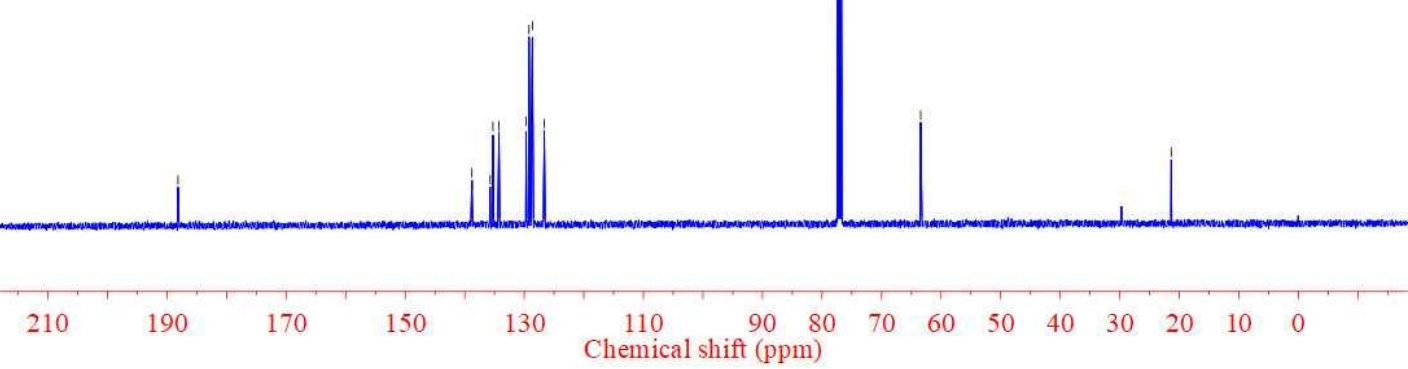

${ }^{13} \mathrm{C}\left\{{ }^{1} \mathrm{H}\right\}$ NMR Spectrum of Compound 3m 


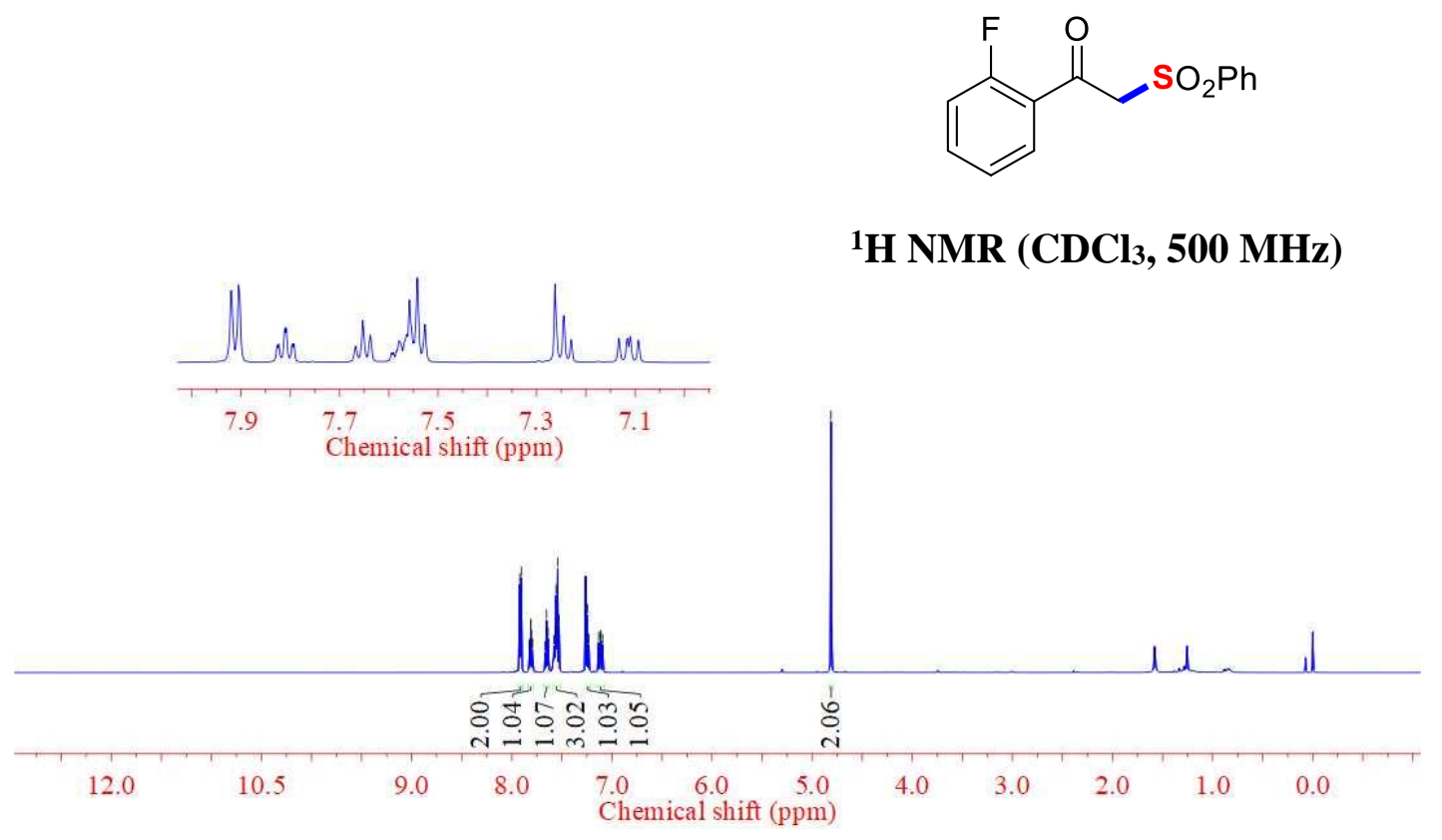

${ }^{1} \mathrm{H}$ NMR Spectrum of Compound 3n

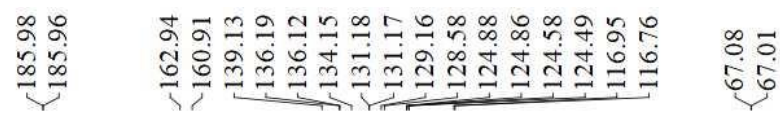<smiles>O=C(CS(=O)(=O)c1ccccc1)c1ccccc1F</smiles>

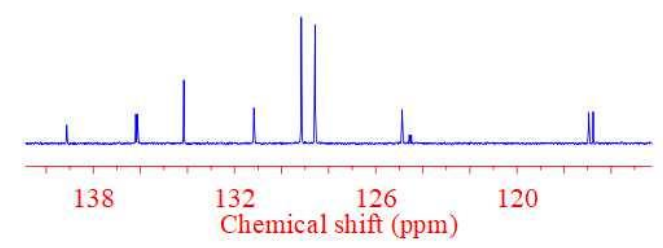

${ }^{13} \mathrm{C}\left\{{ }^{1} \mathrm{H}\right\}$ NMR $\left(\mathrm{CDCl}_{3}, 126 \mathrm{MHz}\right)$

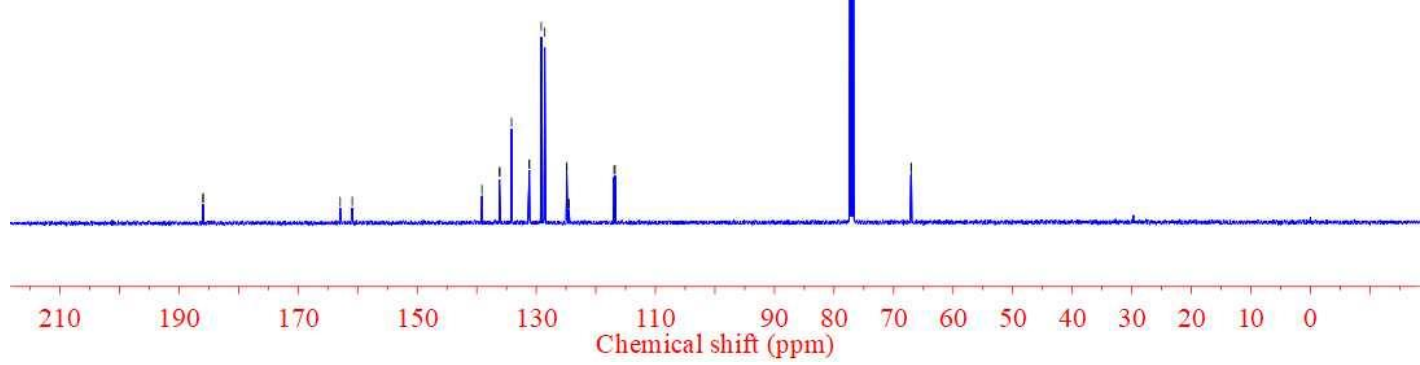

${ }^{13} \mathrm{C}\left\{{ }^{1} \mathrm{H}\right\}$ NMR Spectrum of Compound 3n 
<smiles>O=C(CS(=O)(=O)c1ccccc1)c1ccccc1F</smiles>

${ }^{19} \mathrm{~F}\left\{{ }^{1} \mathrm{H}\right\}$ NMR $\left(\mathrm{CDCl}_{3}, 471 \mathrm{MHz}\right)$

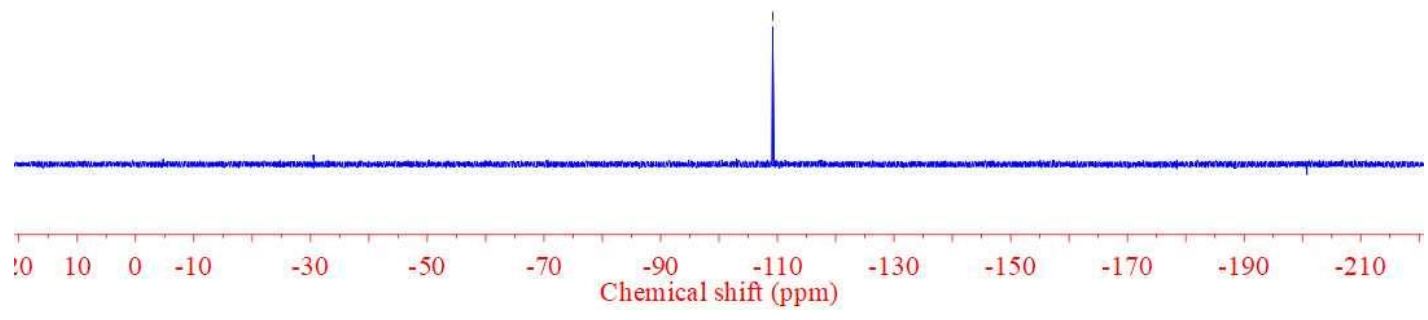

${ }^{19} \mathbf{F}\left\{{ }^{1} \mathbf{H}\right\}$ NMR Spectrum of Compound 3n<smiles>O=C(CS(=O)(=O)c1ccccc1)c1cc(F)ccc1F</smiles>

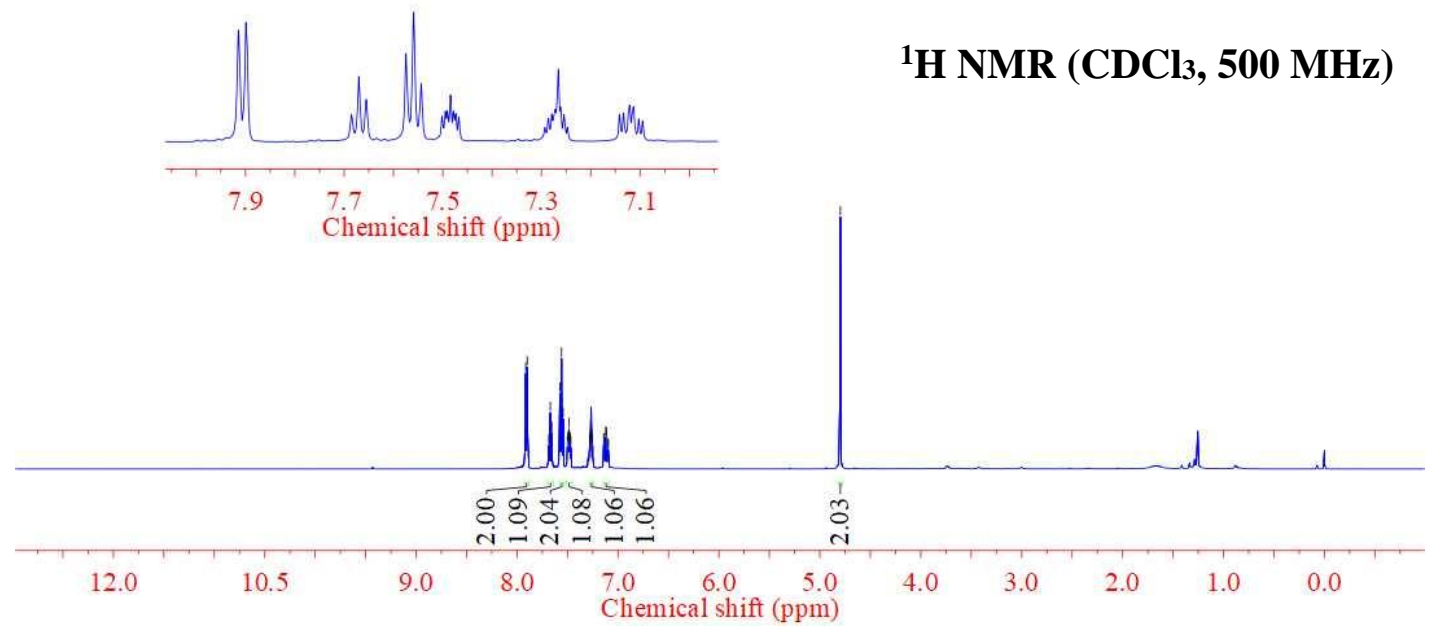

${ }^{1} \mathrm{H}$ NMR (CDCl3, $\left.500 \mathrm{MHz}\right)$

${ }^{1}$ H NMR Spectrum of Compound 30 


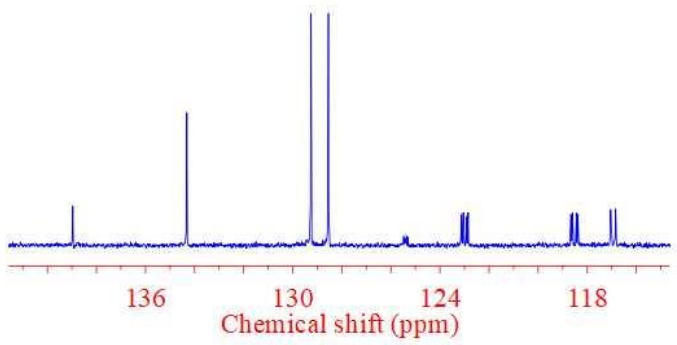<smiles>O=C(CS(=O)(=O)c1ccccc1)c1cc(F)ccc1F</smiles>

${ }^{13} \mathrm{C}\left\{{ }^{1} \mathrm{H}\right\}$ NMR $\left(\mathrm{CDCl}_{3}, 126 \mathrm{MHz}\right)$

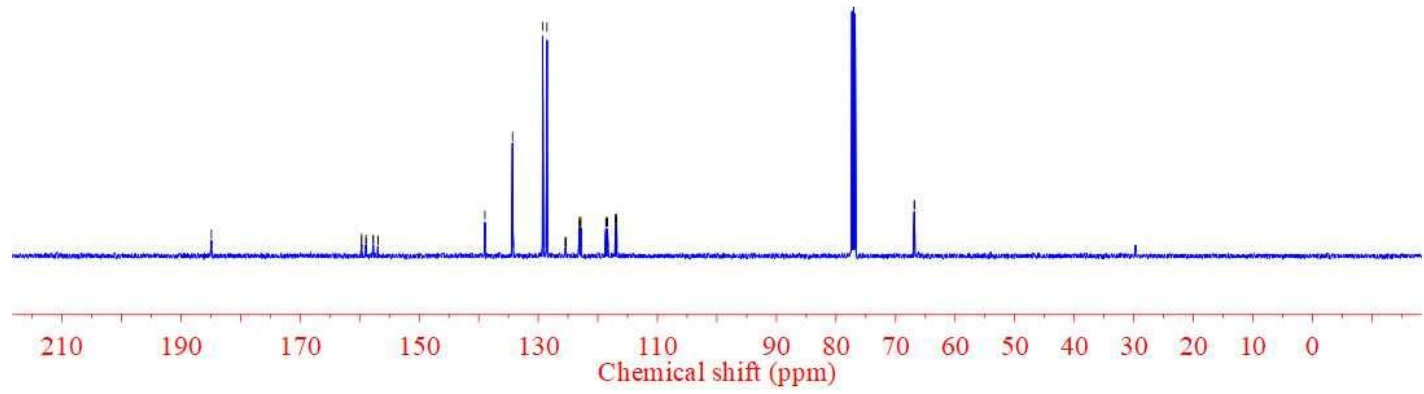

${ }^{13} \mathbf{C}\left\{{ }^{1} \mathrm{H}\right\}$ NMR Spectrum of Compound 30

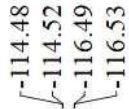<smiles>O=C(CS(=O)(=O)c1ccccc1)c1cc(F)ccc1F</smiles>

${ }^{19} \mathrm{~F}\left\{{ }^{1} \mathrm{H}\right\} \mathrm{NMR}\left(\mathrm{CDCl}_{3}, 471 \mathrm{MHz}\right)$

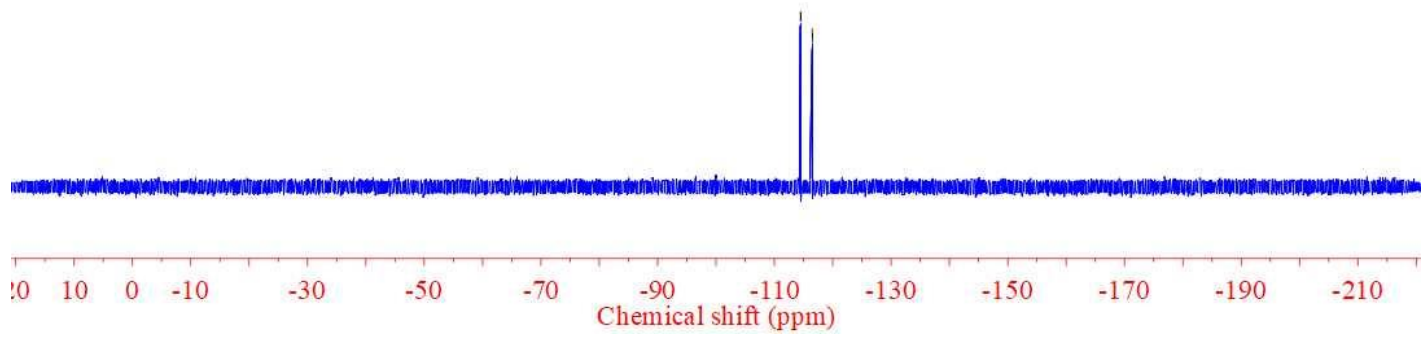

${ }^{19} \mathbf{F}\left\{{ }^{1} \mathbf{H}\right\}$ NMR Spectrum of Compound 30 


\section{Mass Spectrum SmartFormula Report}

Analysis Info

Analysis Name

Method

Sample Name

Comment

Acquisition Date $\quad 8 / 8 / 2020$ 3:31:01 PM

Operator BDAL@DE

Instrument/Ser\# microTOF-Q II 228888.10

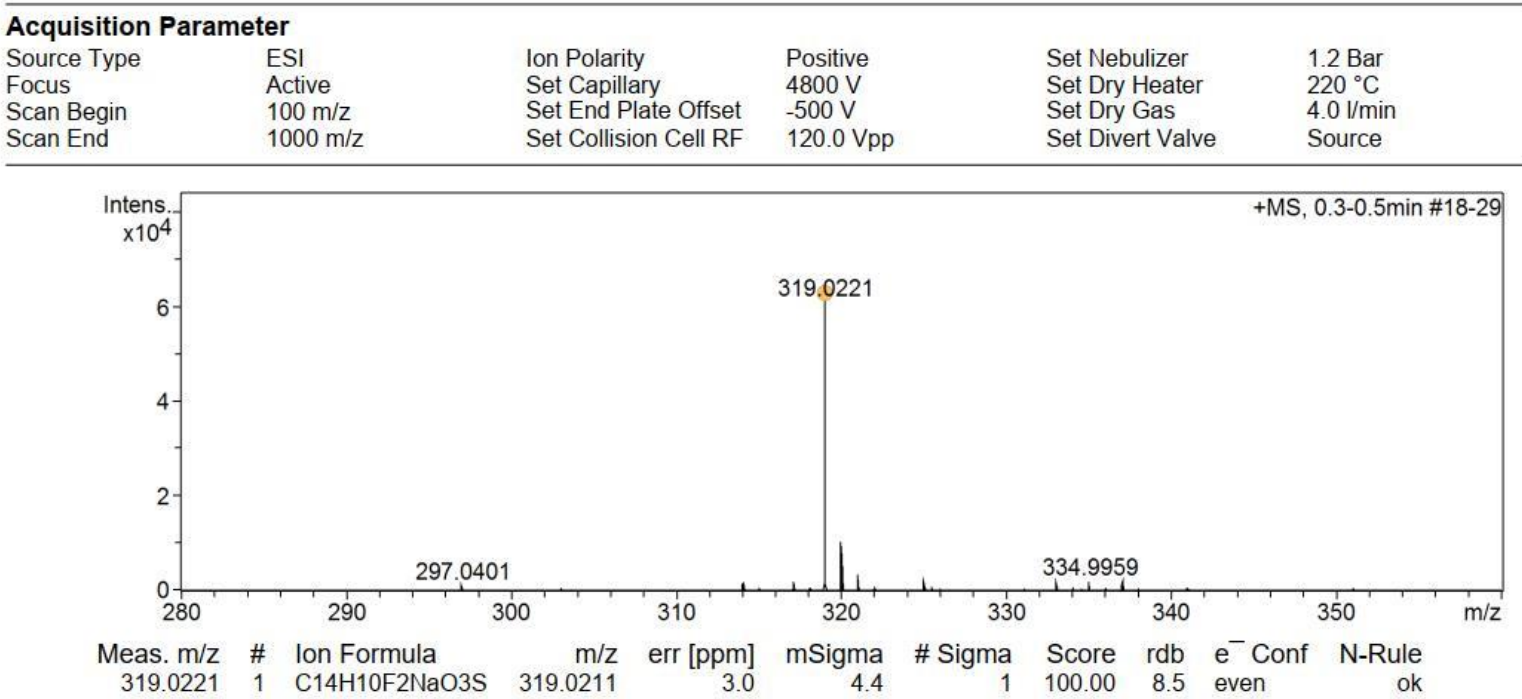

HRMS Spectrum of Compound 30

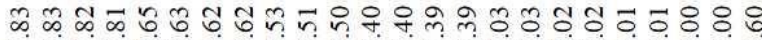

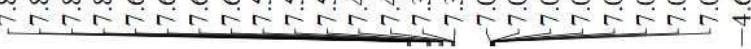<smiles>O=C(CS(=O)(=O)c1ccccc1)c1cc(F)cc(F)c1</smiles>

${ }^{1} \mathrm{H}$ NMR $\left(\mathrm{CDCl}_{3}, 500 \mathrm{MHz}\right)$
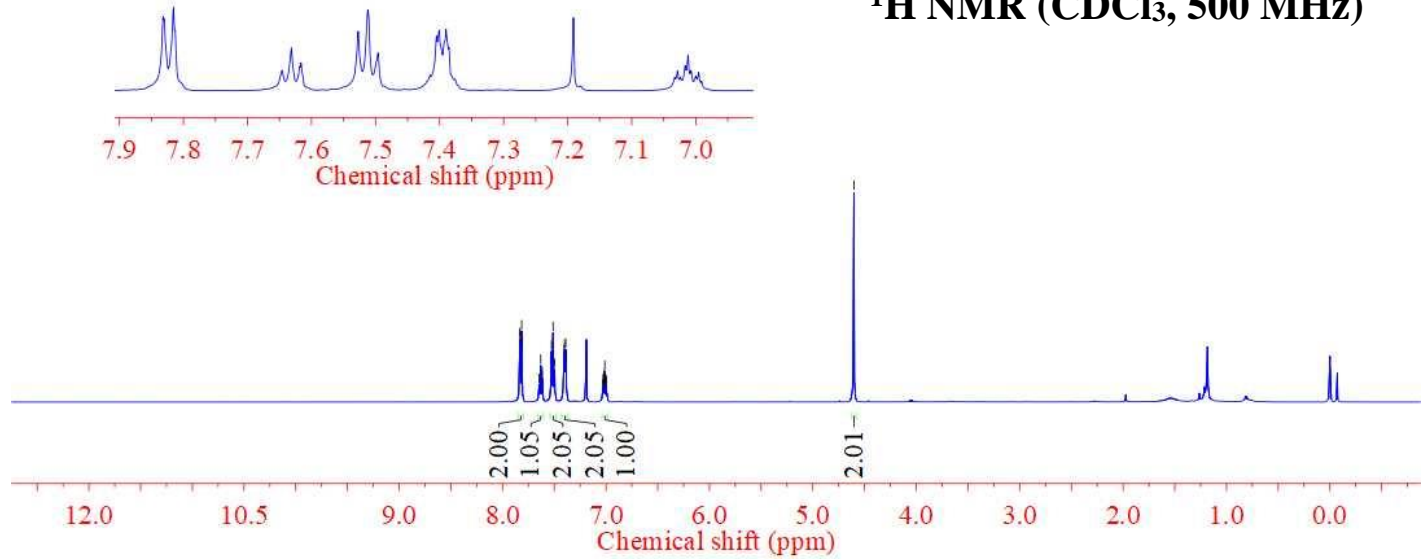

${ }^{1}$ H NMR Spectrum of Compound 3p 
<smiles>O=C(CS(=O)(=O)c1ccccc1)c1cc(F)cc(F)c1</smiles>

${ }^{13} \mathrm{C}\left\{{ }^{1} \mathrm{H}\right\} \mathrm{NMR}\left(\mathrm{CDCl}_{3}, 126 \mathrm{MHz}\right)$
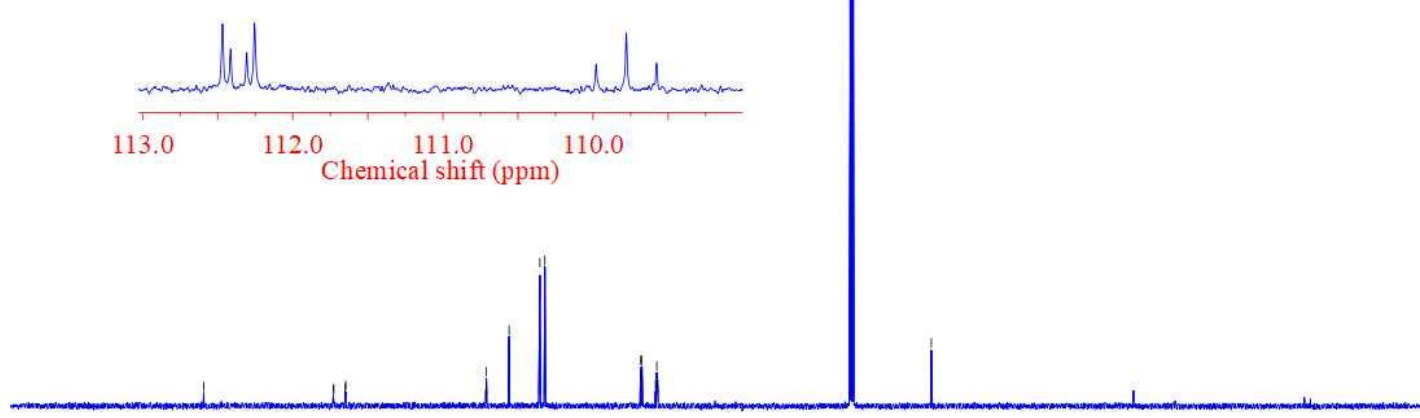

$210-190$

$170 \quad 150 \quad 130$ $\begin{array}{ccccccccccc}110 & 90 & 80 & 70 & 60 & 50 & 40 & 30 & 20 & 10 & 0 \\ \text { Chemical shift (ppm) }\end{array}$

${ }^{13} \mathbf{C}\left\{{ }^{1} \mathrm{H}\right\}$ NMR Spectrum of Compound 3p

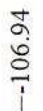<smiles>O=C(CS(=O)(=O)c1ccccc1)c1cc(F)cc(F)c1</smiles>

${ }^{19} \mathrm{~F}\left\{{ }^{1} \mathrm{H}\right\}$ NMR $\left(\mathrm{CDCl}_{3}, 471 \mathrm{MHz}\right)$

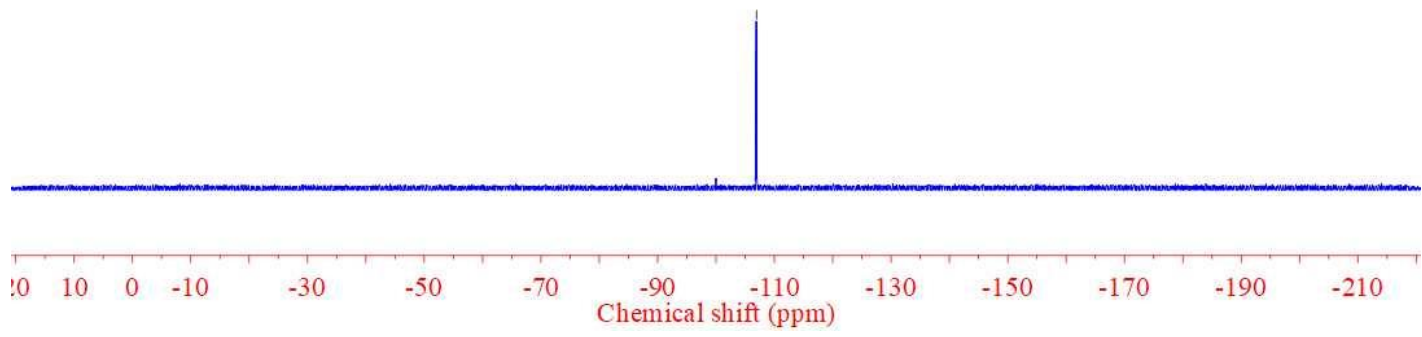

${ }^{19} \mathbf{F}\left\{{ }^{1} \mathbf{H}\right\}$ NMR Spectrum of Compound 3p 
<smiles>O=C(CS(=O)(=O)c1ccccc1)c1cc(F)c(F)c(F)c1</smiles>

${ }^{1} \mathrm{H}$ NMR $\left(\mathrm{CDCl}_{3}, 500 \mathrm{MHz}\right)$
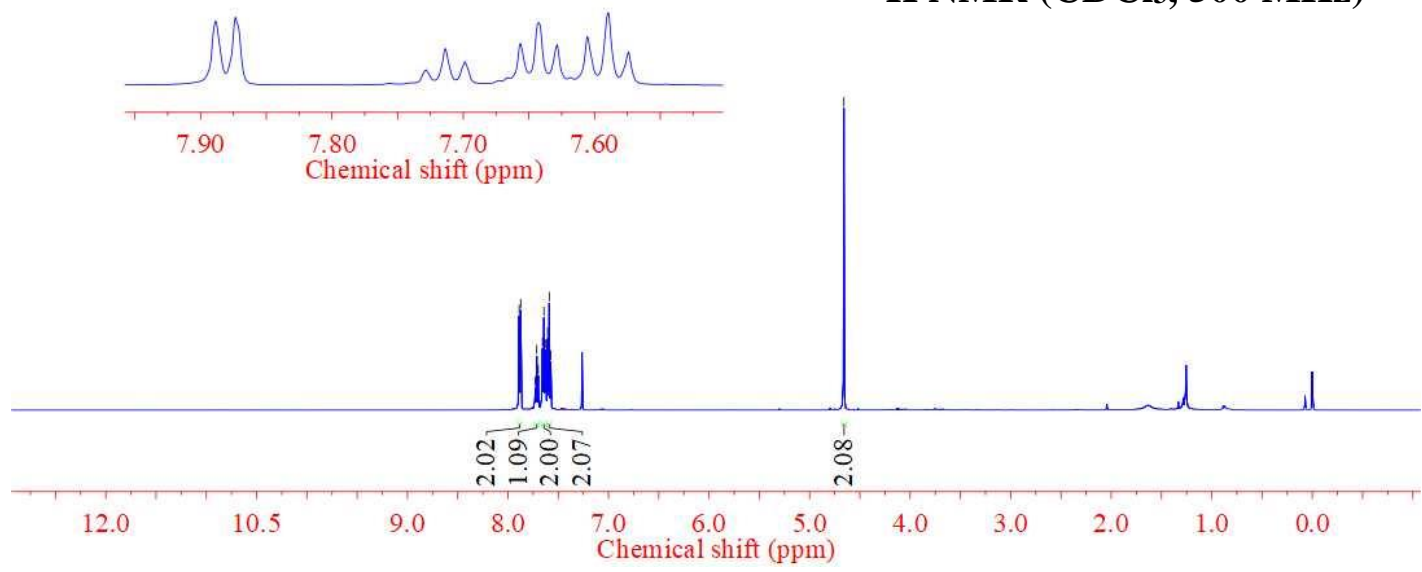

${ }^{1} \mathrm{H}$ NMR Spectrum of Compound 3q

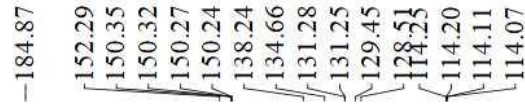
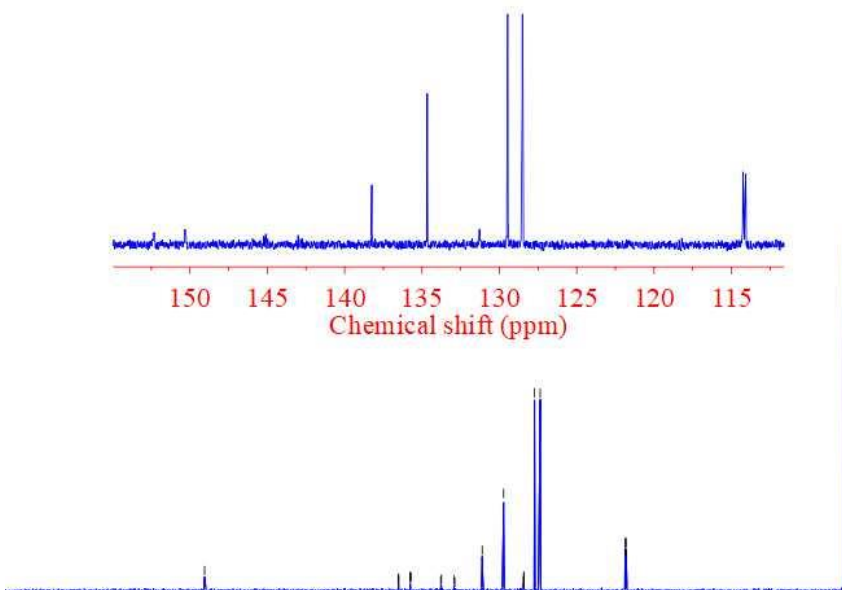

210

190

150

130
盀<smiles>O=C(CS(=O)(=O)c1ccccc1)c1cc(F)c(F)c(F)c1</smiles>

${ }^{13} \mathrm{C}\left\{{ }^{1} \mathrm{H}\right\}$ NMR $(\mathrm{CDCl} 3,126 \mathrm{MHz})$

${ }^{13} \mathrm{C}\left\{{ }^{1} \mathrm{H}\right\}$ NMR Spectrum of Compound 3q 
<smiles>O=C(CS(=O)(=O)c1ccccc1)c1cc(F)c(F)c(F)c1</smiles>

${ }^{19} \mathrm{~F}\left\{{ }^{1} \mathrm{H}\right\}$ NMR $\left(\mathrm{CDCl}_{3}, 471 \mathrm{MHz}\right)$

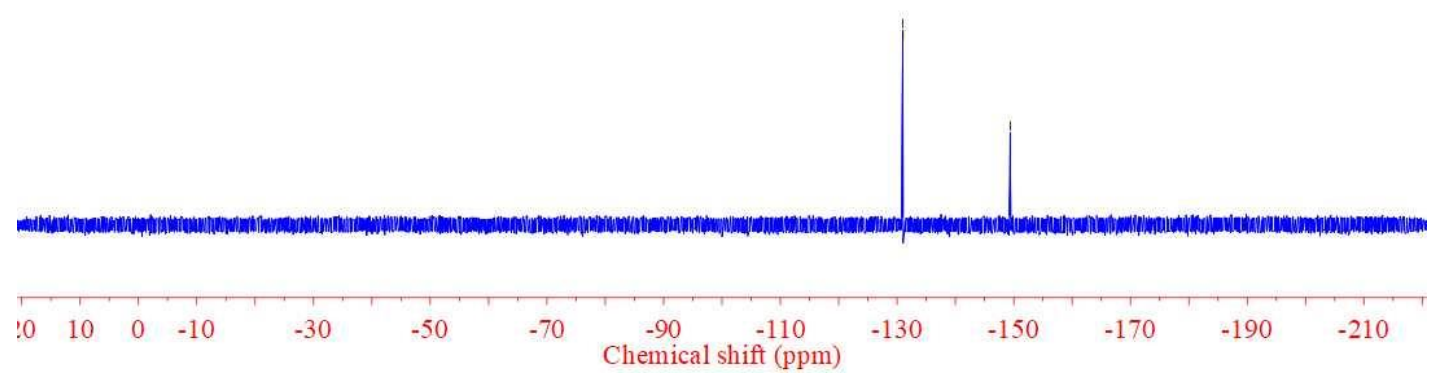

${ }^{19} \mathbf{F}\left\{{ }^{1} \mathbf{H}\right\}$ NMR Spectrum of Compound 3q

\section{Mass Spectrum SmartFormula Report}

\section{Analysis Info}

Analysis Name

Method

Sample Name

Comment
Acquisition Date $\quad 9 / 11 / 2020$ 9:40:22 AM

Operator BDAL@DE

Instrument microTOF-Q II 228888.10324

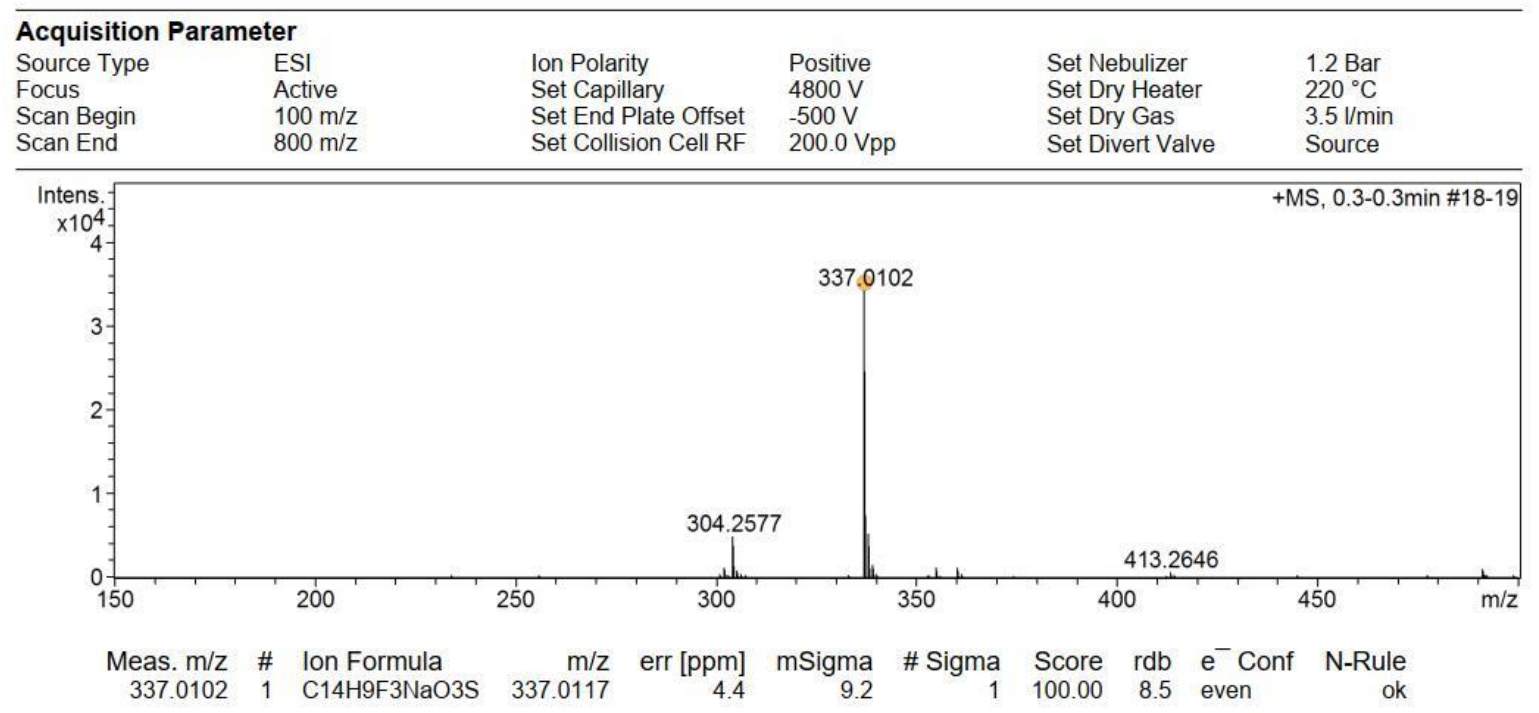

HRMS Spectrum of Compound 3q 


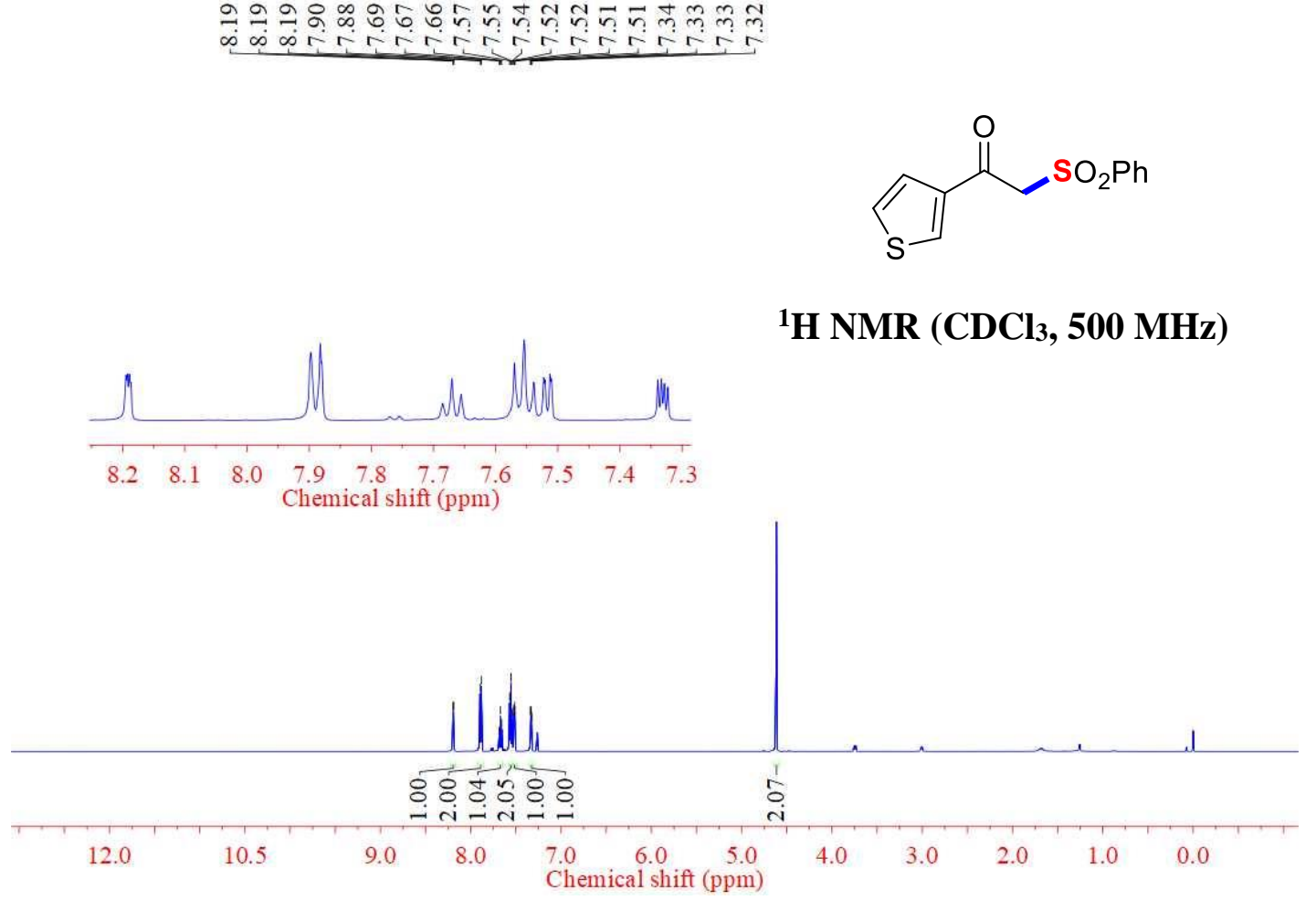

${ }^{1}$ H NMR Spectrum of Compound 3r

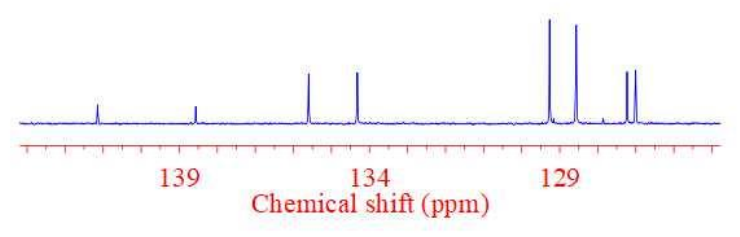

$\frac{0}{10}$<smiles>O=C(CS(=O)(=O)c1ccccc1)c1ccsc1</smiles>

${ }^{13} \mathrm{C}\left\{{ }^{1} \mathrm{H}\right\}$ NMR $\left(\mathrm{CDCl}_{3}, 126 \mathrm{MHz}\right)$

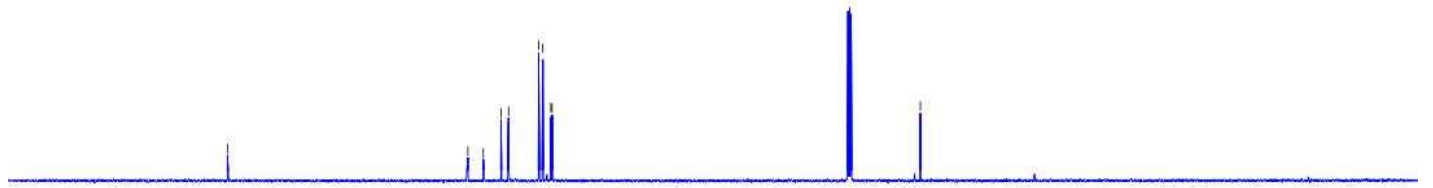

$210 \quad 190 \quad 170$

150

$130 \quad 110$

$\begin{array}{lllllllll}90 & 80 & 70 & 60 & 50 & 40 & 30 & 20 & 10\end{array}$

${ }^{13} \mathbf{C}\left\{{ }^{1} \mathbf{H}\right\}$ NMR Spectrum of Compound $3 \mathbf{r}$ 
<smiles>CC(C(=O)c1ccccc1)[Sb](=O)(O)c1ccccc1</smiles>
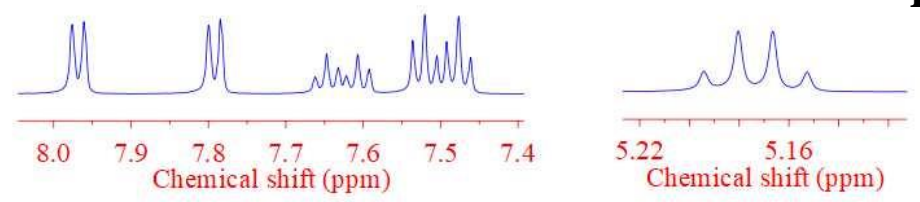

${ }^{1} \mathrm{H}$ NMR (CDCl3, $\left.500 \mathrm{MHz}\right)$

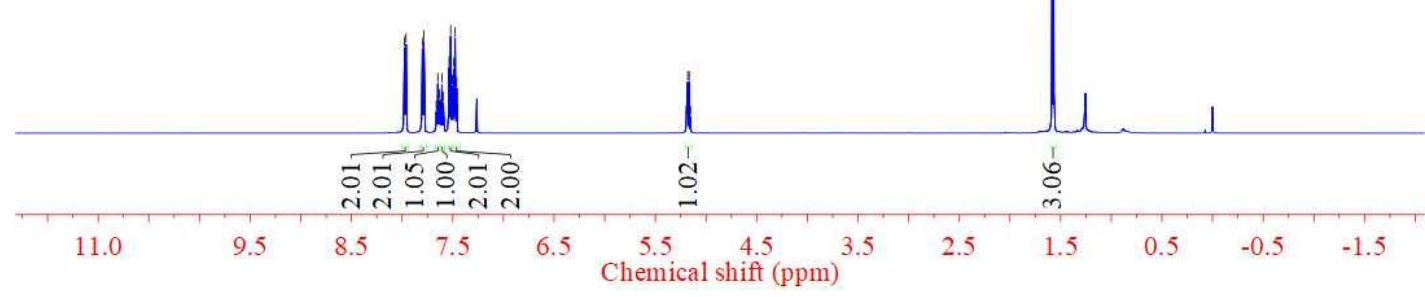

${ }^{1} \mathrm{H}$ NMR Spectrum of Compound 3s
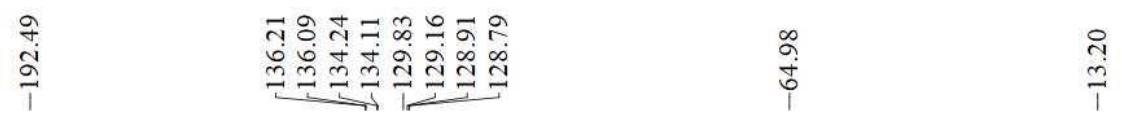<smiles>CC(C(=O)c1ccccc1)S(=O)(=O)c1ccccc1</smiles>

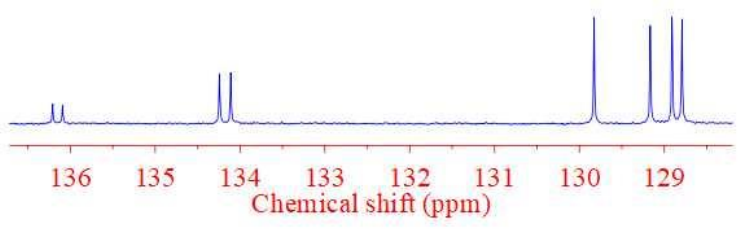

${ }^{13} \mathrm{C}\left\{{ }^{1} \mathrm{H}\right\}$ NMR $\left(\mathrm{CDCl}_{3}, 126 \mathrm{MHz}\right)$
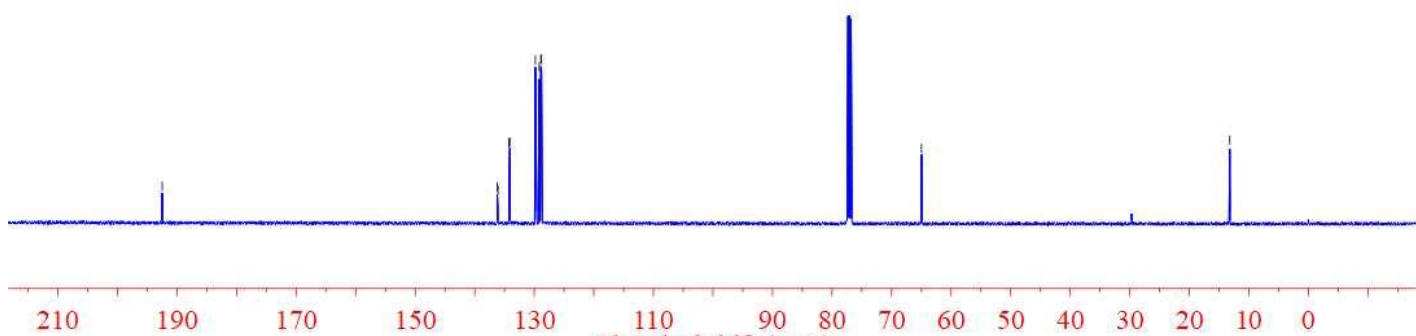

${ }^{13} \mathrm{C}\left\{{ }^{1} \mathrm{H}\right\}$ NMR Spectrum of Compound 3s 
<smiles>CCC(C(=O)c1ccccc1)S(=O)(=O)c1ccccc1</smiles>

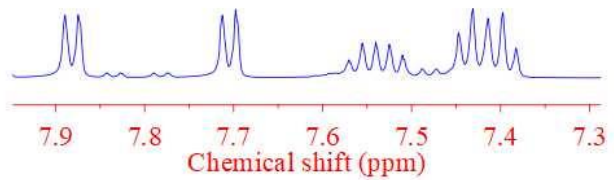

${ }^{1} \mathrm{H}$ NMR $\left(\mathrm{CDCl}_{3}, 500 \mathrm{MHz}\right)$

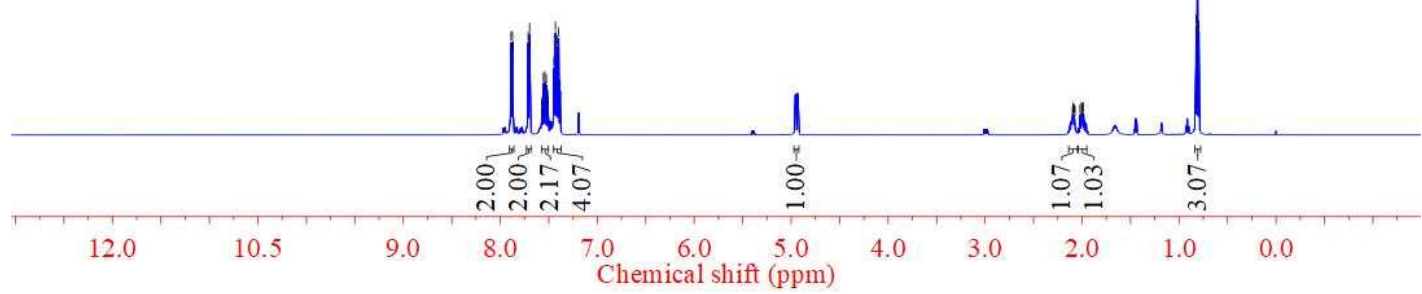

${ }^{1}$ H NMR Spectrum of Compound $3 \mathrm{t}$

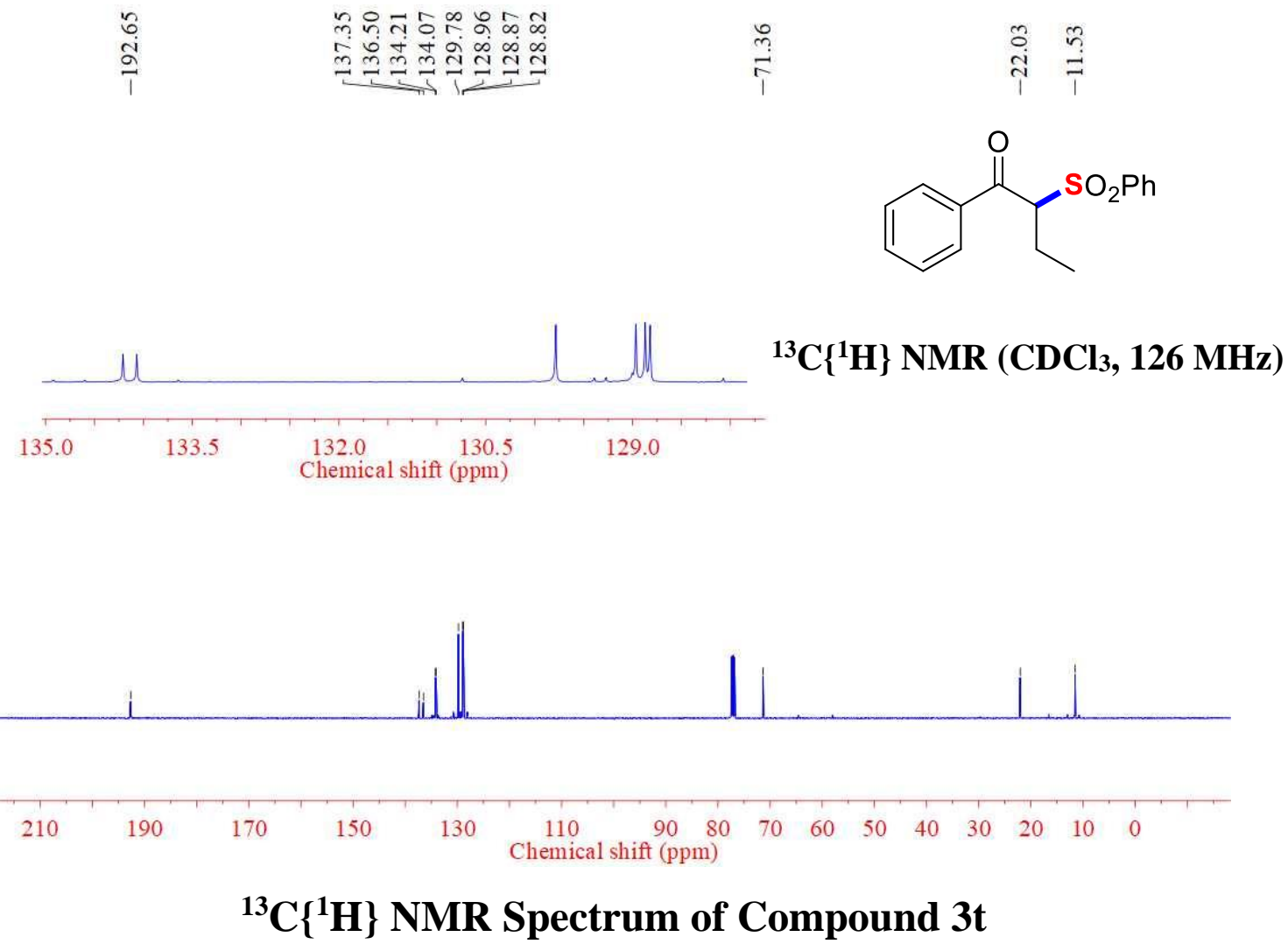


<smiles>CCCC(C(=O)c1ccccc1)S(=O)(=O)c1ccccc1</smiles>

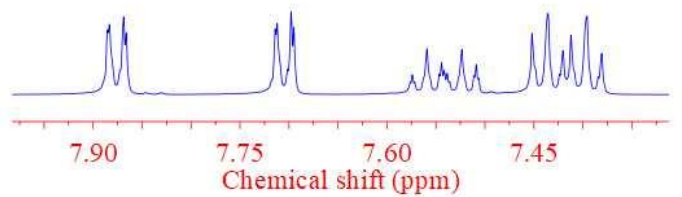

${ }^{1} \mathrm{H}$ NMR $\left(\mathrm{CDCl}_{3}, 500 \mathrm{MHz}\right)$

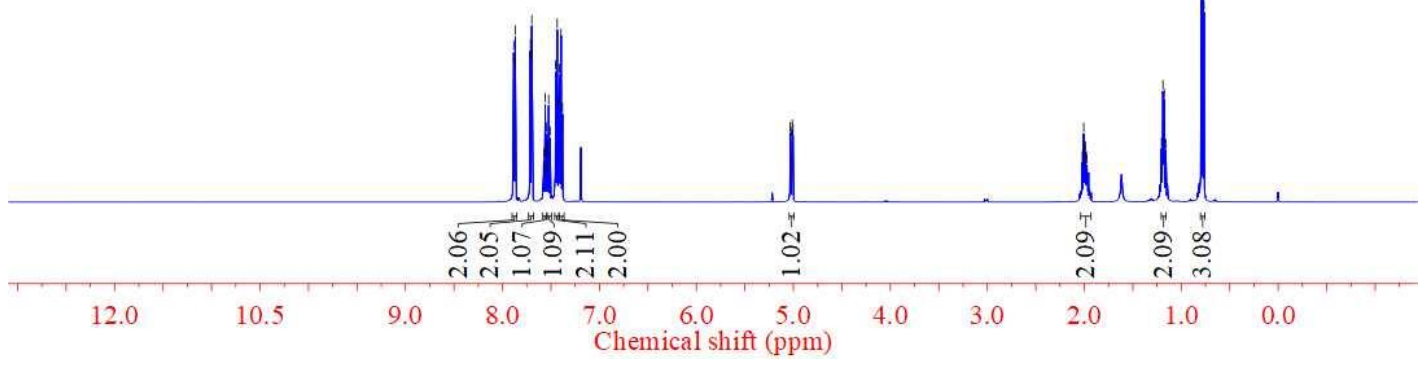

${ }^{1}$ H NMR Spectrum of Compound 3u

ลั่

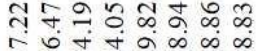

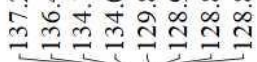

๙ิণ

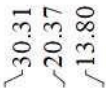<smiles>CCCC(C(=O)c1ccccc1)S(=O)(=O)c1ccccc1</smiles>

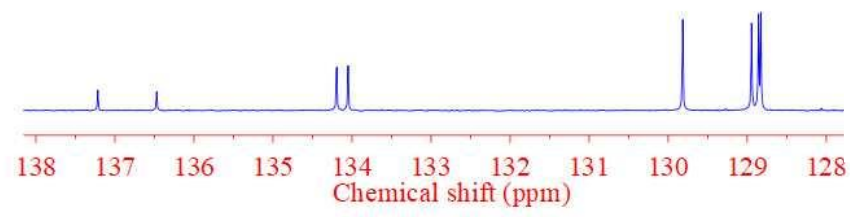

${ }^{13} \mathrm{C}\left\{{ }^{1} \mathrm{H}\right\}$ NMR (CDCl3, $\left.126 \mathrm{MHz}\right)$

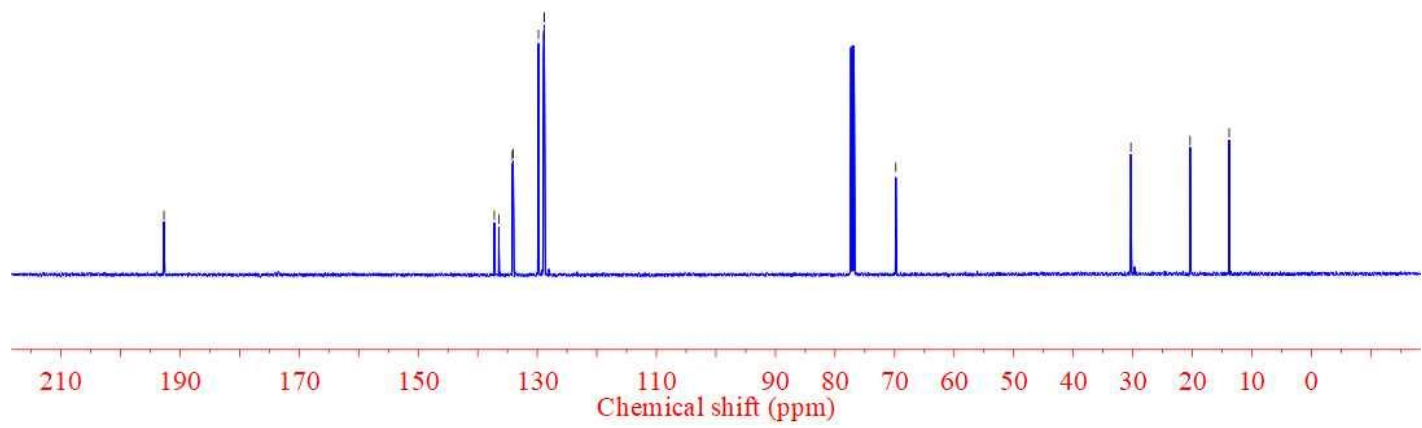

${ }^{13} \mathrm{C}\left\{{ }^{1} \mathrm{H}\right\}$ NMR Spectrum of Compound $3 \mathrm{u}$ 


\section{Mass Spectrum SmartFormula Report}

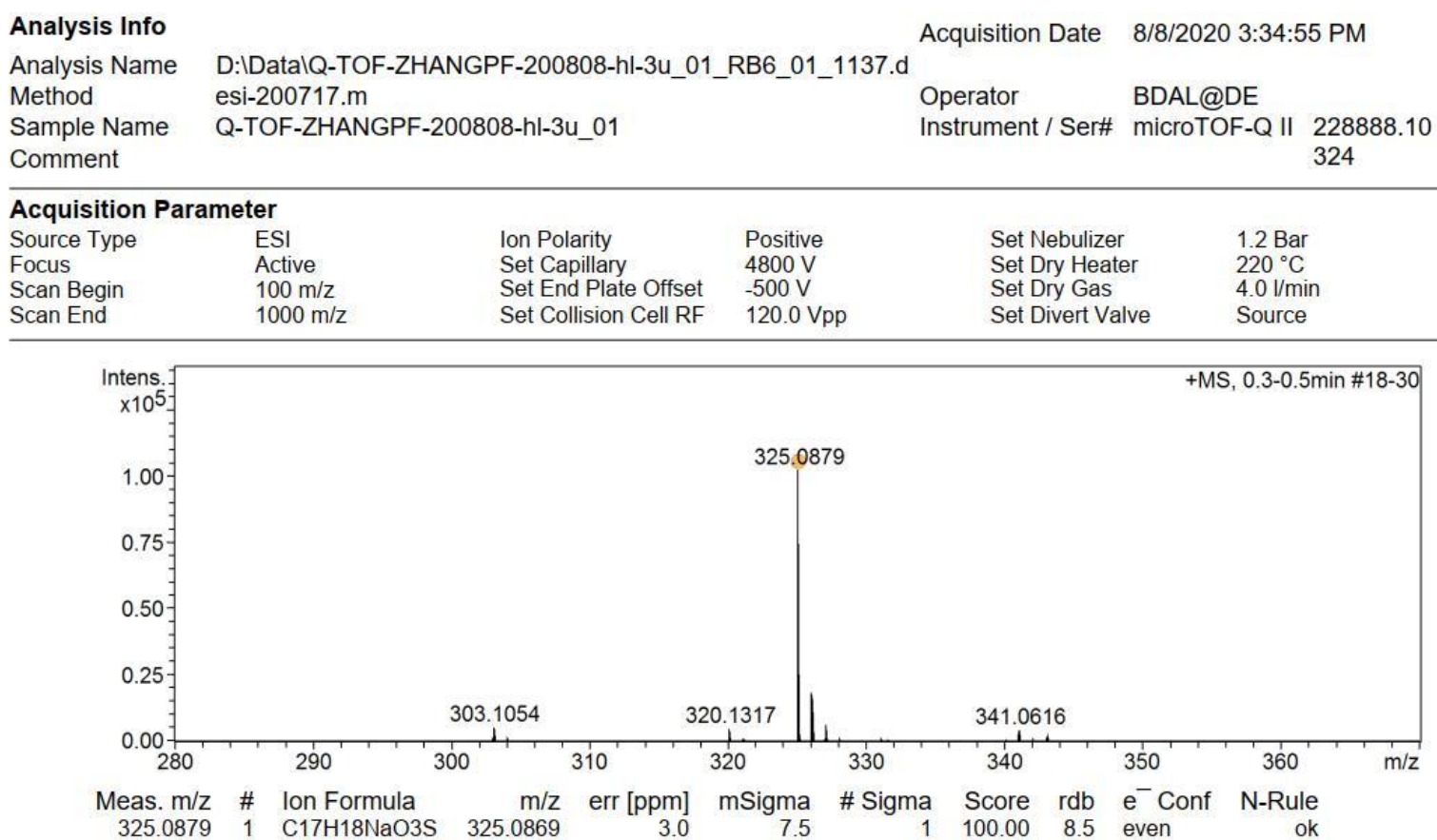

HRMS Spectrum of Compound 3u
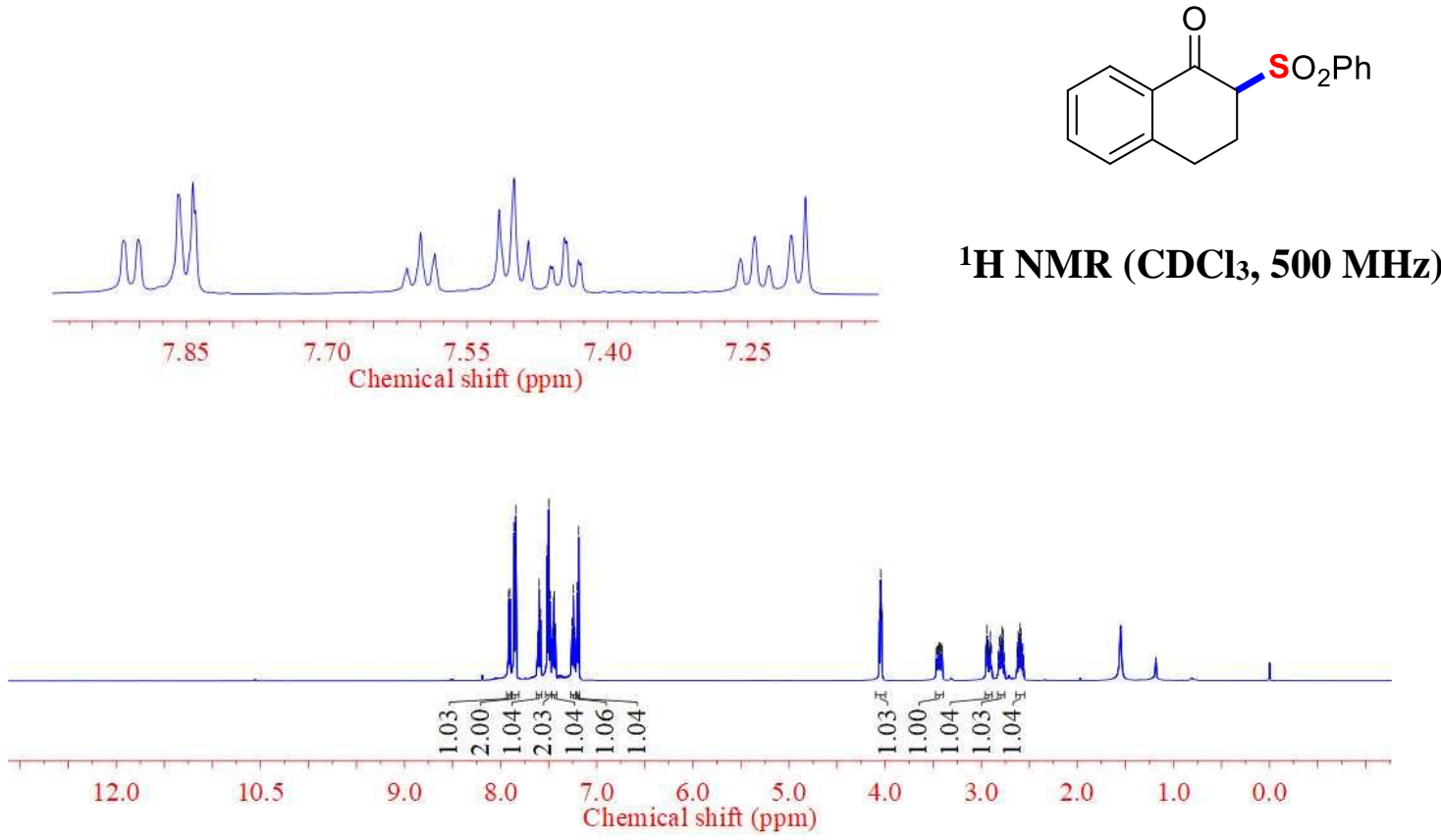

${ }^{1} \mathrm{H}$ NMR Spectrum of Compound 3v 

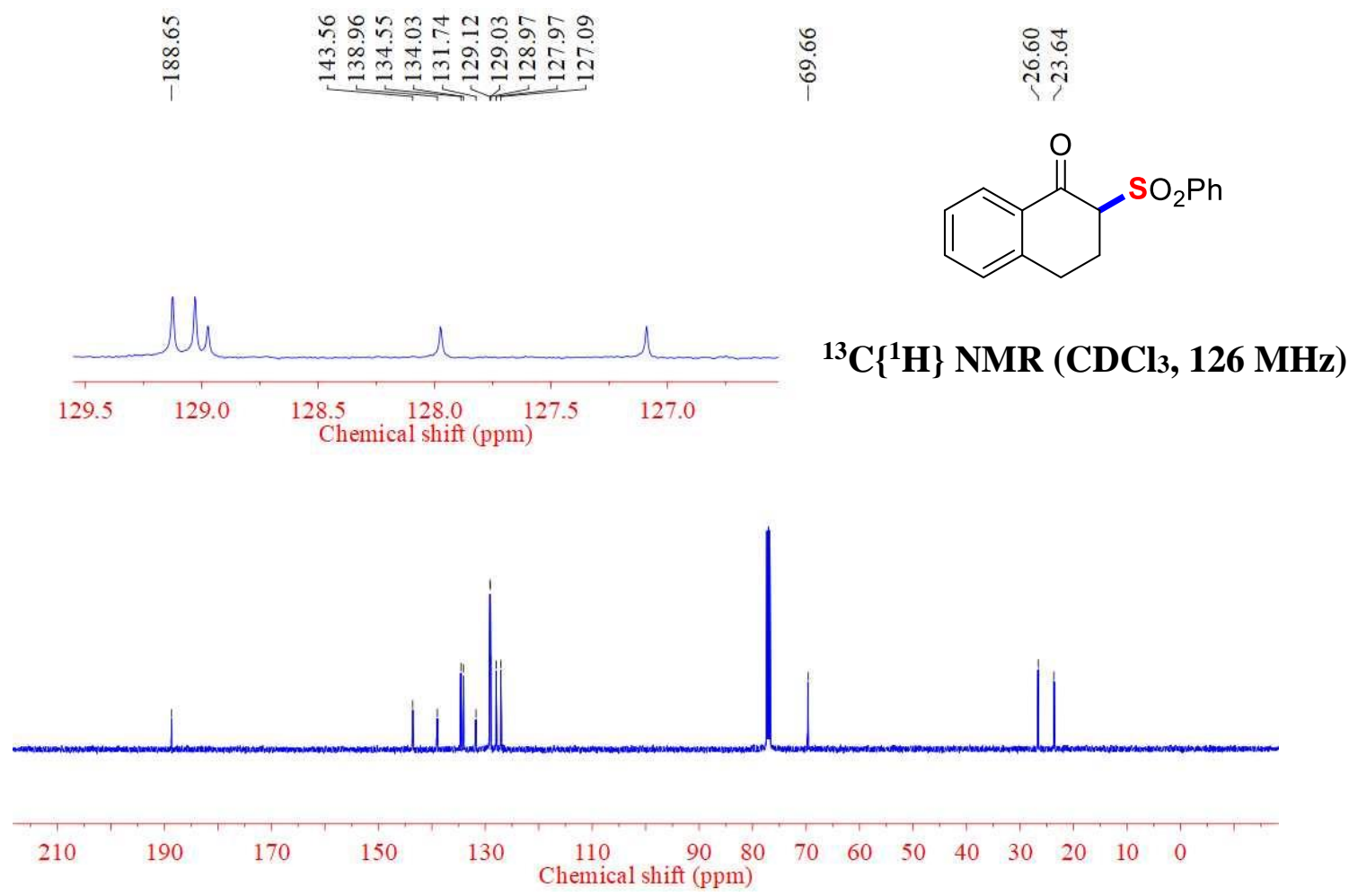

${ }^{13} \mathrm{C}\left\{{ }^{1} \mathrm{H}\right\}$ NMR Spectrum of Compound $3 \mathrm{v}$

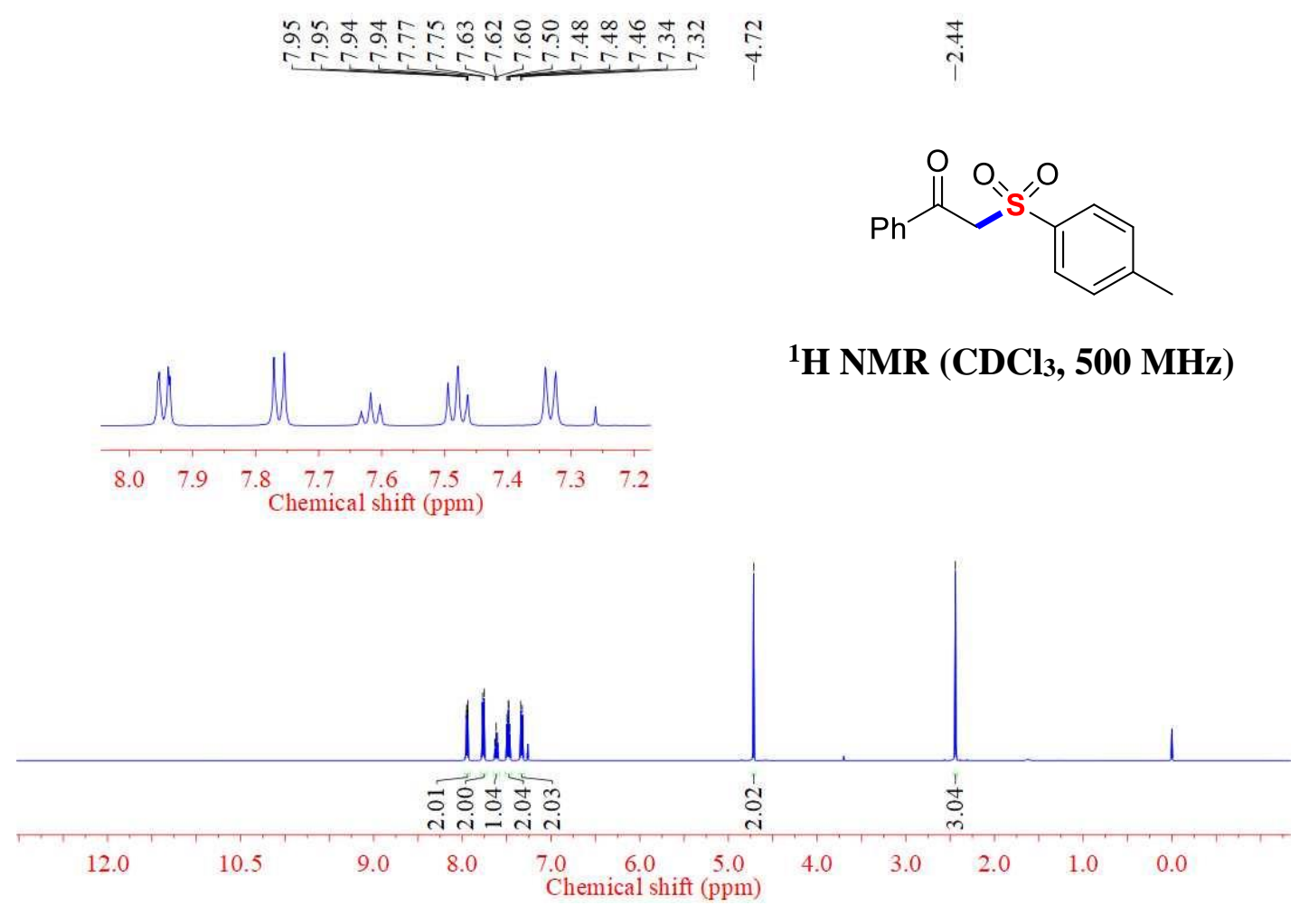

${ }^{1} H$ NMR Spectrum of Compound 3x 


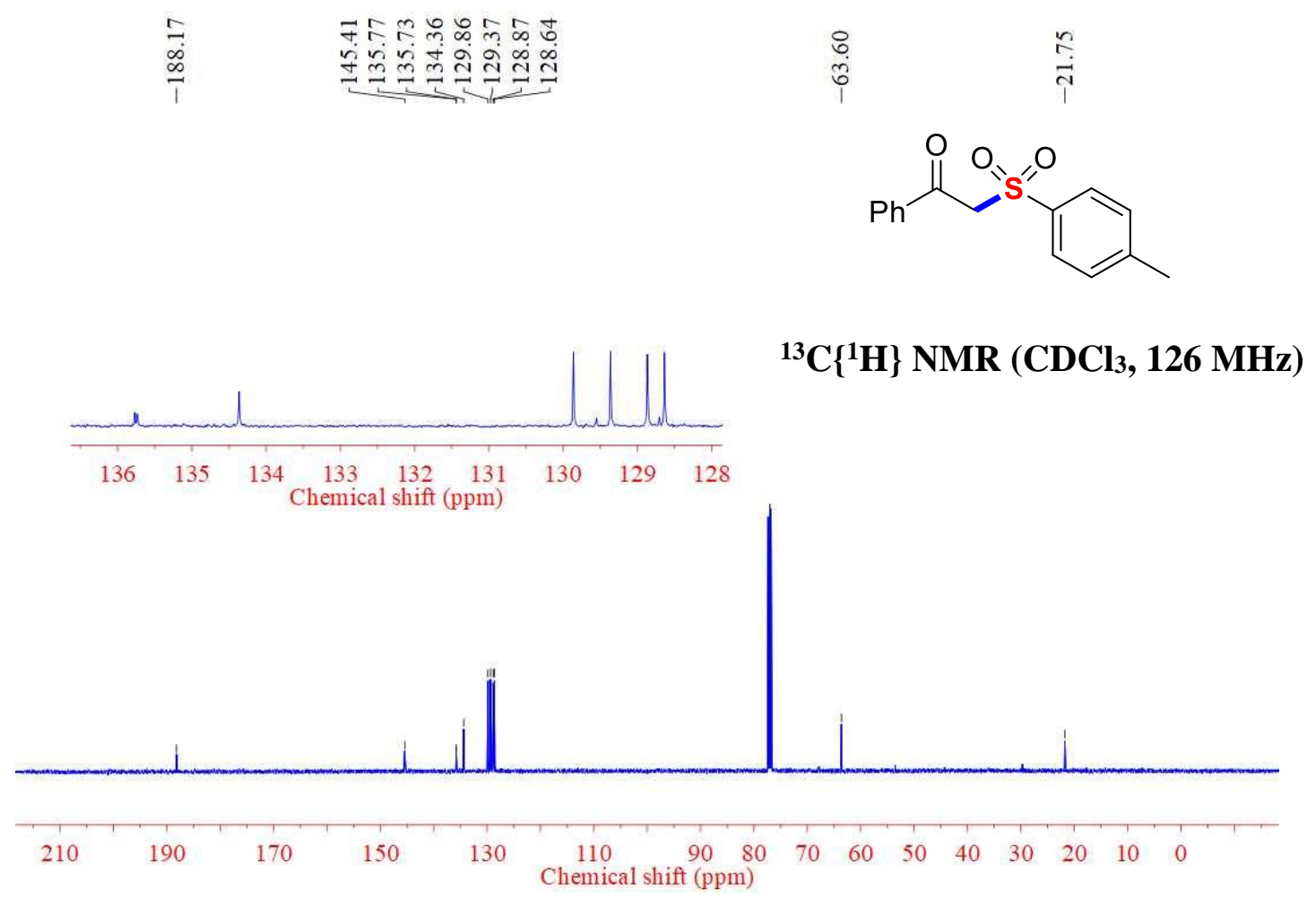

\section{${ }^{13} \mathbf{C}\left\{{ }^{1} \mathbf{H}\right\}$ NMR Spectrum of Compound $3 x$}

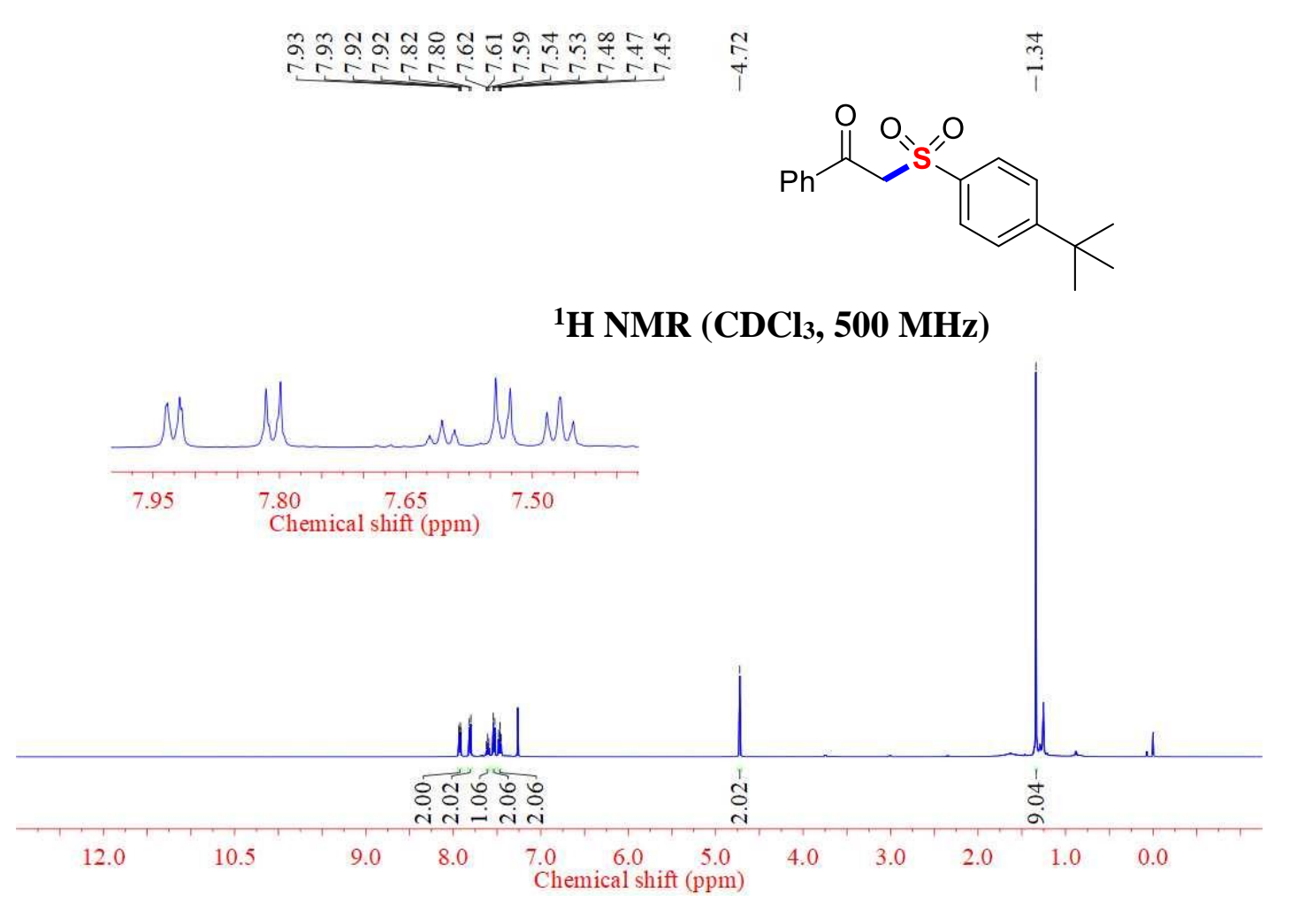

${ }^{1}$ H NMR Spectrum of Compound 3y 


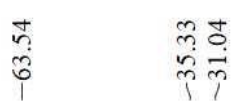<smiles>CC(C)(C)c1ccc(S(=O)(=O)CC(=O)c2ccccc2)cc1</smiles>

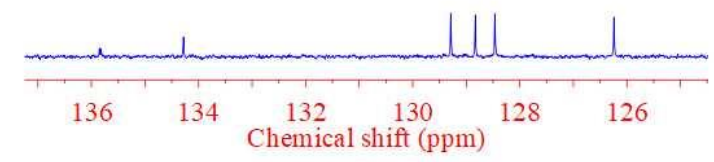

${ }^{13} \mathrm{C}\left\{{ }^{1} \mathrm{H}\right\} \mathrm{NMR}(\mathrm{CDCl}, 126 \mathrm{MHz})$

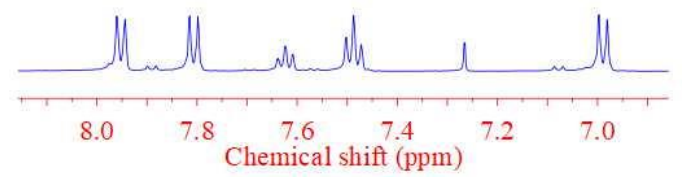

${ }^{1} \mathrm{H}$ NMR $\left(\mathrm{CDCl}_{3}, 500 \mathrm{MHz}\right)$

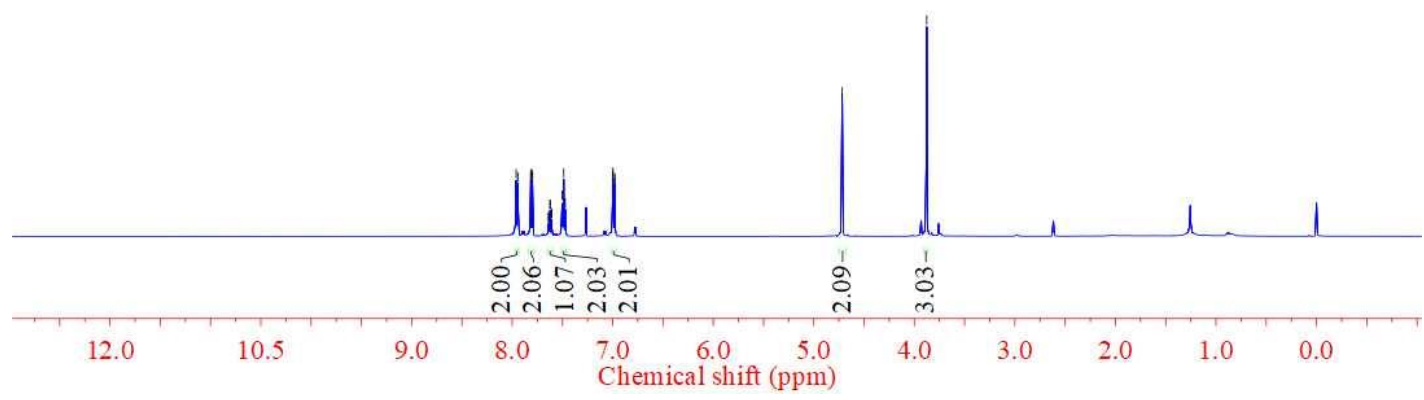

${ }^{1} \mathrm{H}$ NMR Spectrum of Compound 3z 
<smiles>COc1ccc(S(=O)(=O)CC(=O)c2ccccc2)cc1</smiles>

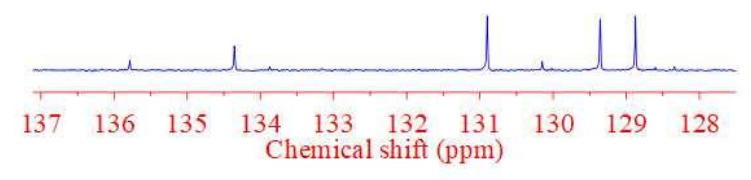

${ }^{13} \mathrm{C}\left\{{ }^{1} \mathrm{H}\right\} \mathrm{NMR}\left(\mathrm{CDCl}_{3}, 126 \mathrm{MHz}\right)$

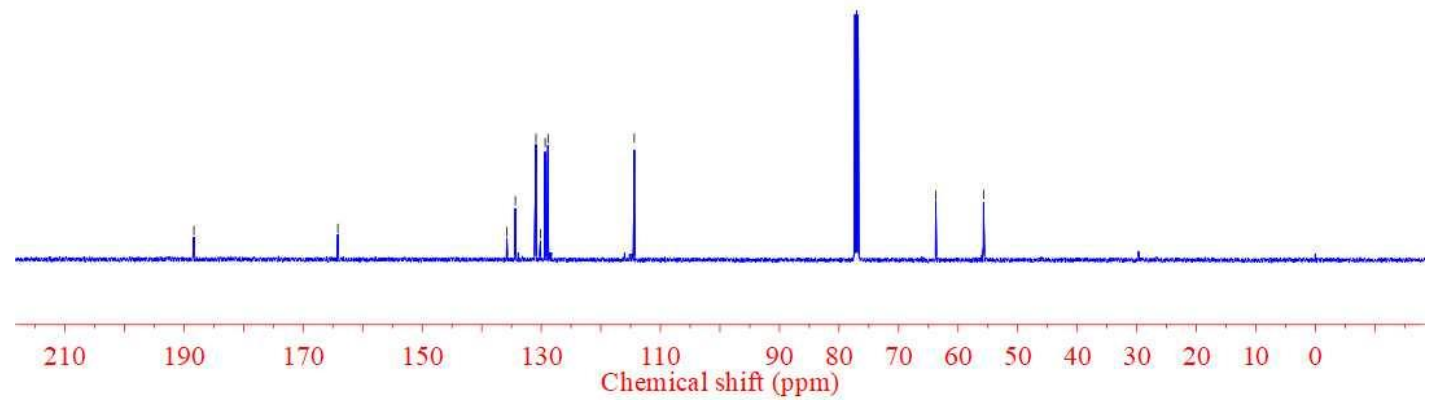

${ }^{13} \mathbf{C}\left\{{ }^{1} \mathrm{H}\right\}$ NMR Spectrum of Compound $3 z$

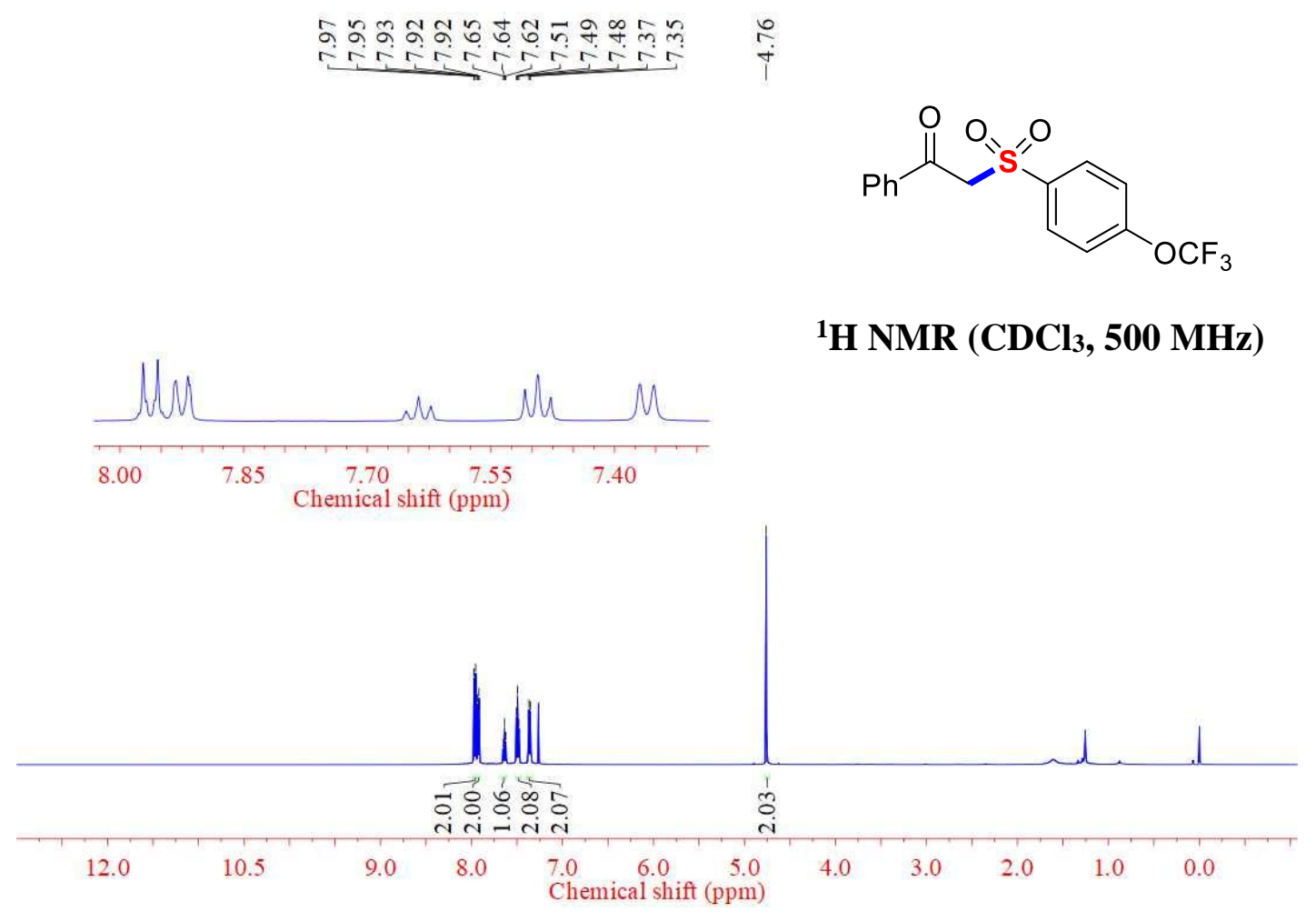

${ }^{1} \mathrm{H}$ NMR Spectrum of Compound 3aa 


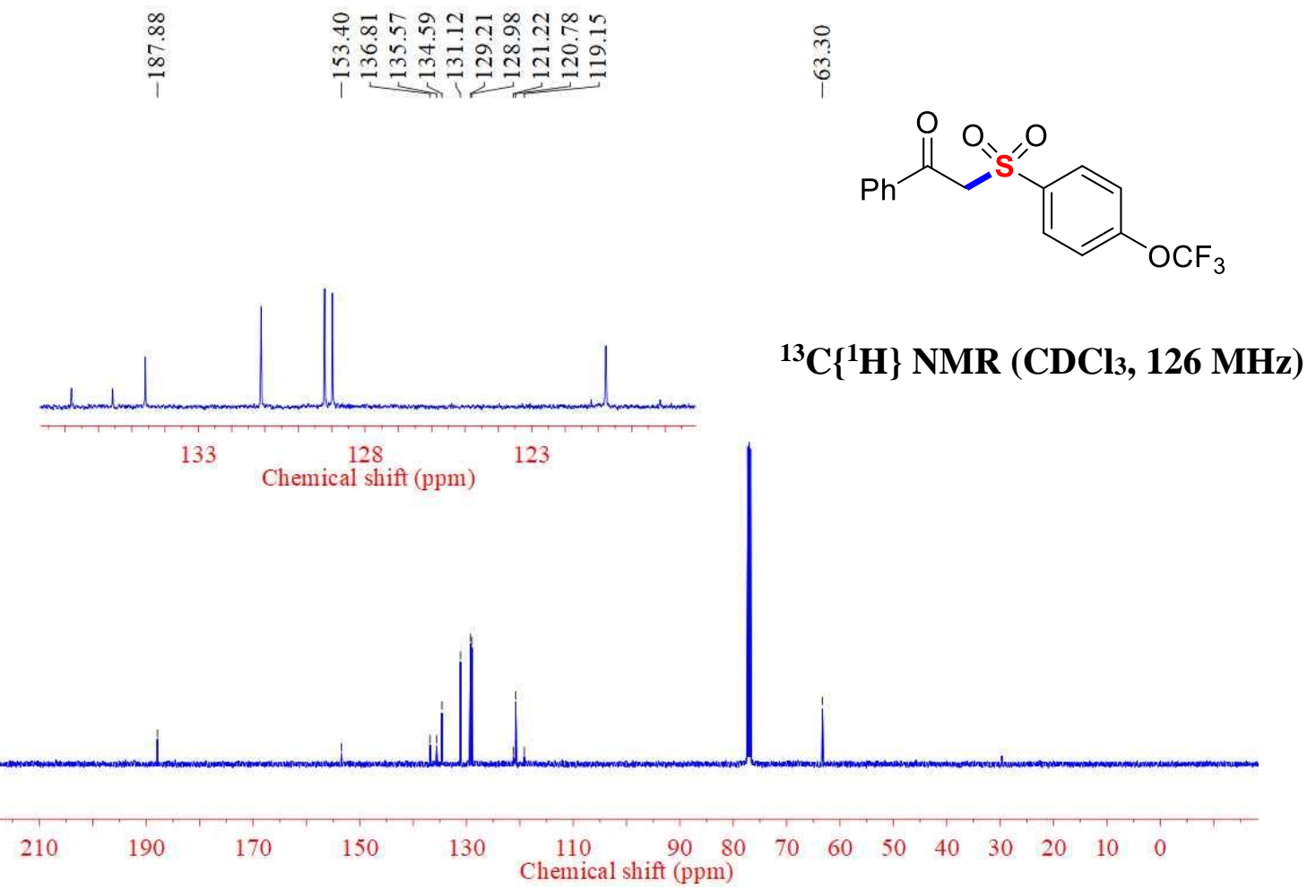

${ }^{13} \mathbf{C}\left\{{ }^{1} \mathbf{H}\right\}$ NMR Spectrum of Compound 3aa

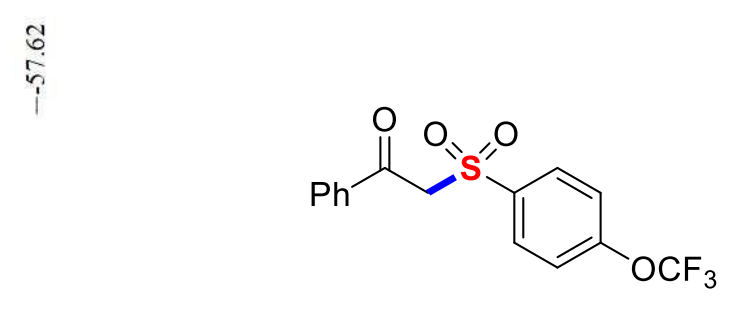

${ }^{19} \mathrm{~F}\left\{{ }^{1} \mathrm{H}\right\}$ NMR $\left(\mathrm{CDCl}_{3}, 471 \mathrm{MHz}\right)$

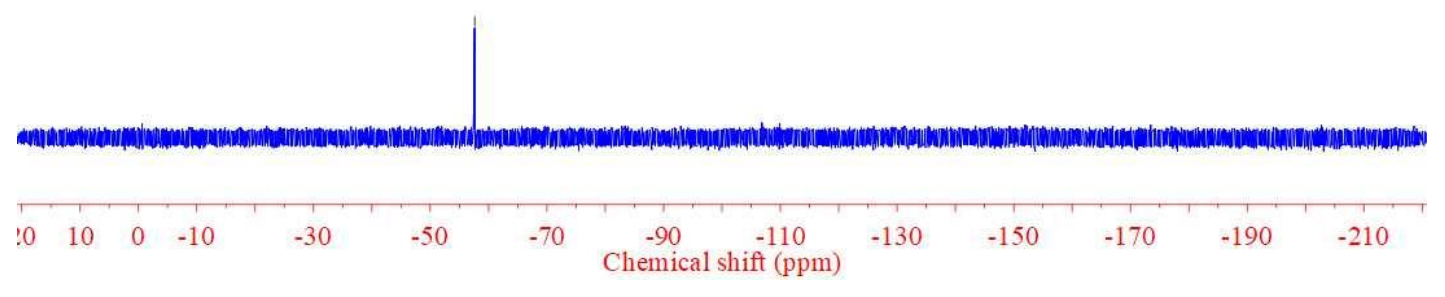

${ }^{19} \mathbf{F}\left\{{ }^{1} \mathrm{H}\right\}$ NMR Spectrum of Compound 3aa 


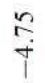<smiles>O=C(CS(=O)(=O)c1ccc(Cl)cc1)c1ccccc1</smiles>

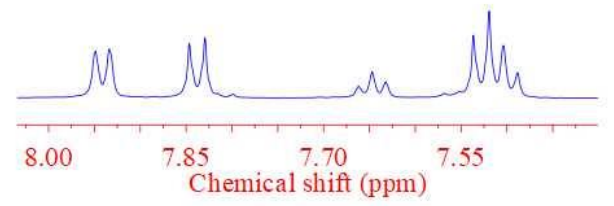

${ }^{1} \mathrm{H}$ NMR (CDCl3, $\left.500 \mathrm{MHz}\right)$

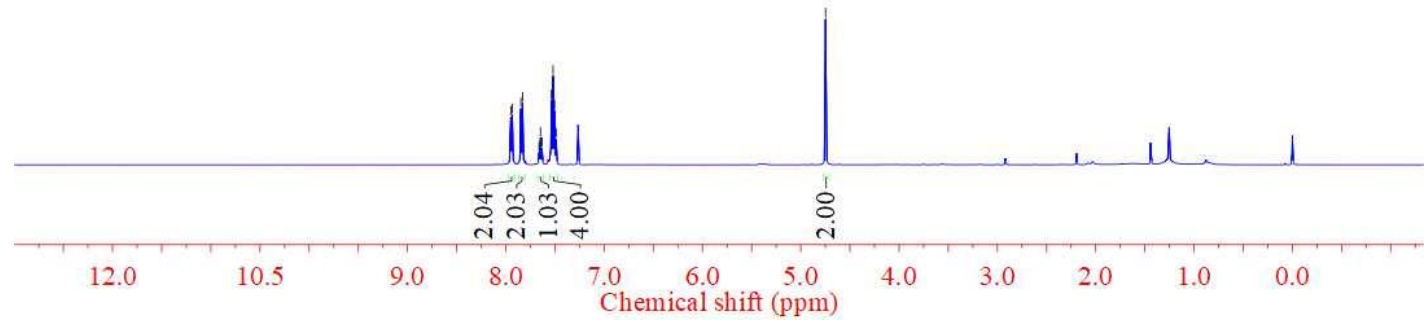

${ }^{1} \mathrm{H}$ NMR Spectrum of Compound 3ab

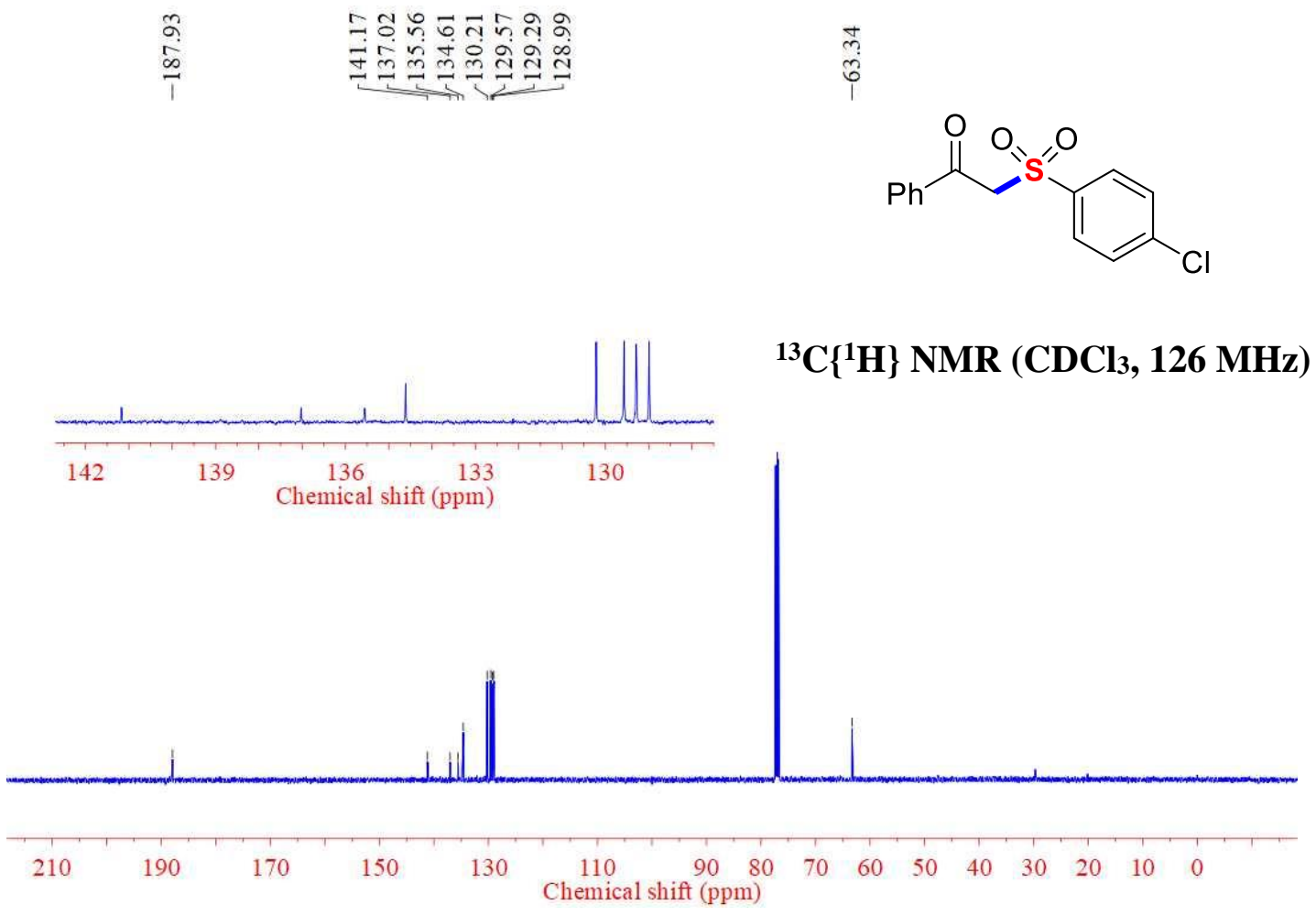

${ }^{13} \mathbf{C}\left\{{ }^{1} \mathbf{H}\right\}$ NMR Spectrum of Compound 3ab 
<smiles>O=C(CS(=O)(=O)c1ccc(Br)cc1)c1ccccc1</smiles>

${ }^{1} \mathrm{H}$ NMR (CDCl3, $\left.500 \mathrm{MHz}\right)$
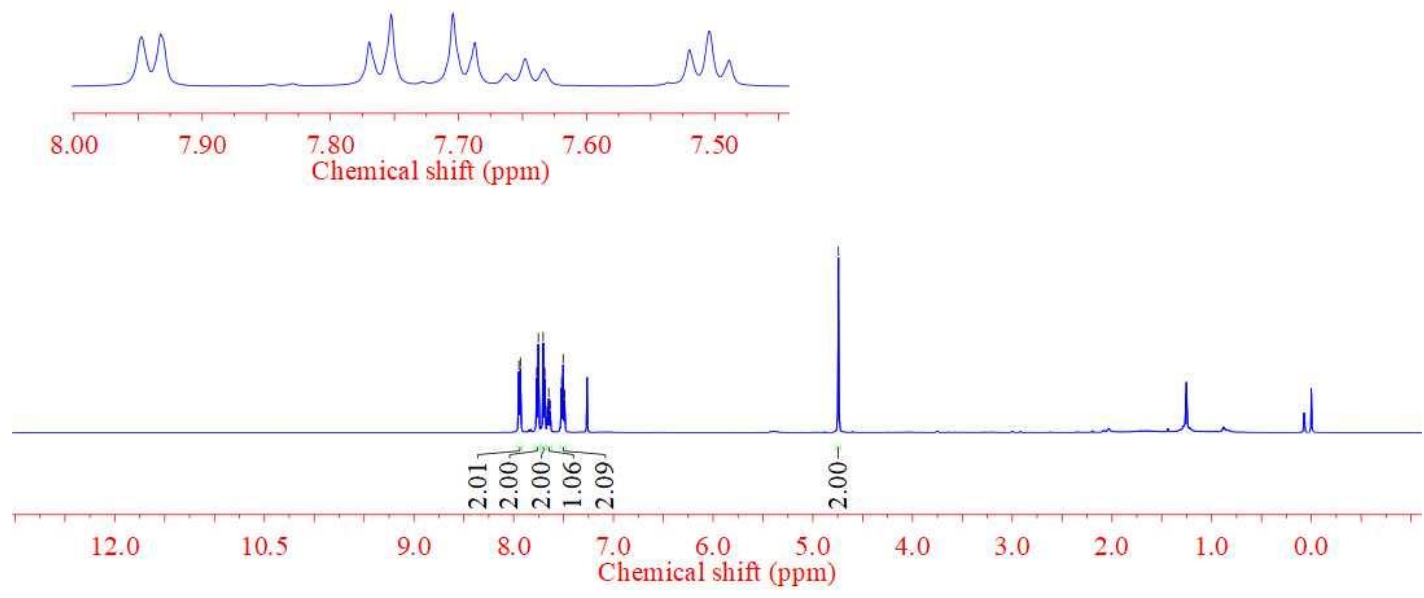

${ }^{1} \mathrm{H}$ NMR Spectrum of Compound 3ac

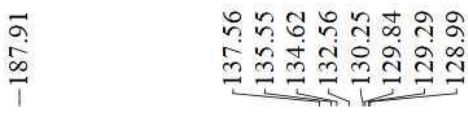

$\vec{m}$<smiles>O=C(CS(=O)(=O)c1ccc(Br)cc1)c1ccccc1</smiles>

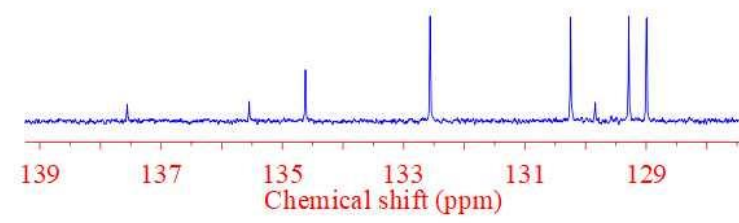

${ }^{13} \mathrm{C}\left\{{ }^{1} \mathrm{H}\right\} \mathrm{NMR}\left(\mathrm{CDCl}_{3}, 126 \mathrm{MHz}\right)$

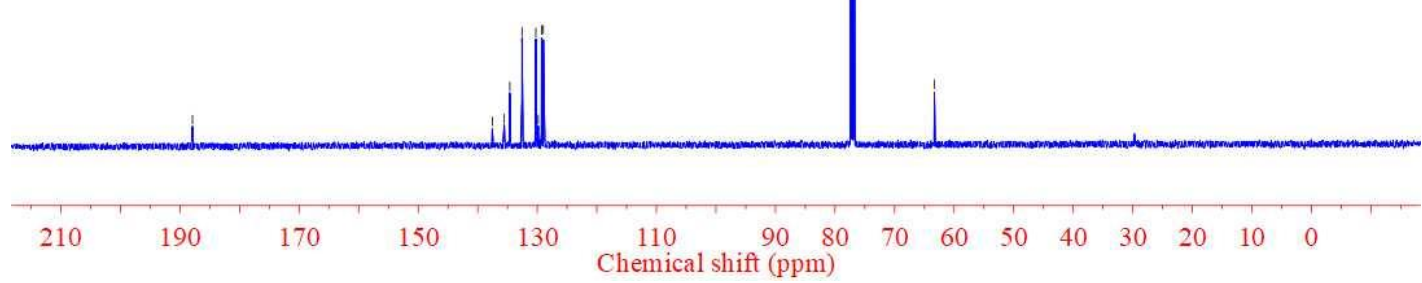

${ }^{13} \mathrm{C}\left\{{ }^{1} \mathrm{H}\right\}$ NMR Spectrum of Compound 3ac 
<smiles>CCOC(=O)c1ccc(S(=O)(=O)CC(=O)c2ccccc2)cc1</smiles>

${ }^{1} \mathrm{H}$ NMR $\left(\mathrm{CDCl}_{3}, 500 \mathrm{MHz}\right)$
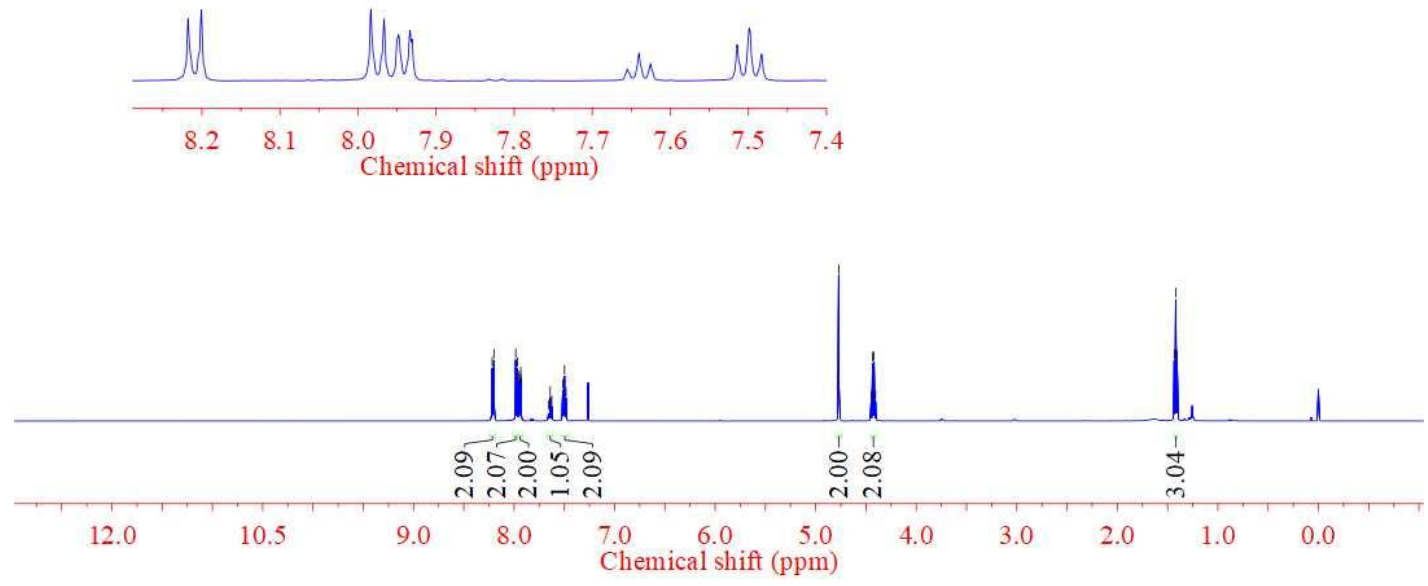

${ }^{1}$ H NMR Spectrum of Compound 3ad

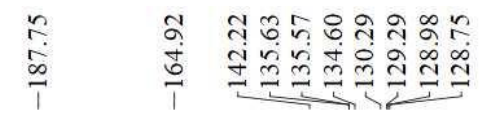
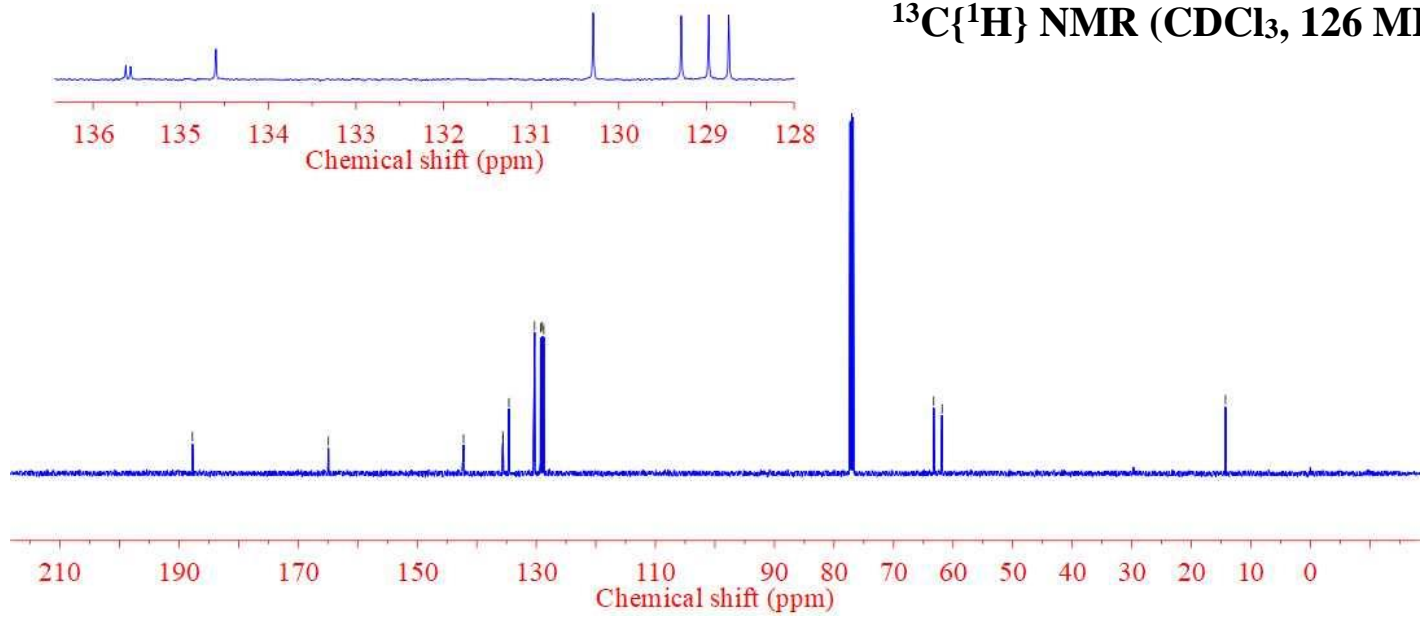

${ }^{13} \mathrm{C}\left\{{ }^{1} \mathrm{H}\right\}$ NMR Spectrum of Compound 3ad

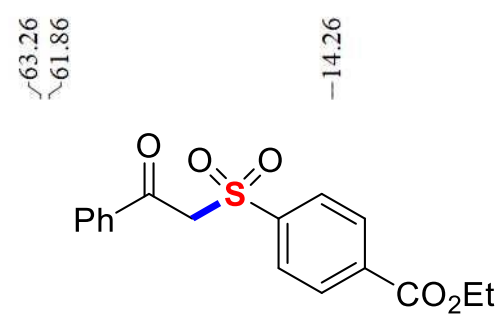

${ }^{13} \mathrm{C}\left\{{ }^{1} \mathrm{H}\right\}$ NMR $\left(\mathrm{CDCl}_{3}, 126 \mathrm{MHz}\right)$ 
<smiles>Cc1cccc(S(=O)(=O)CC(=O)c2ccccc2)c1</smiles>

${ }^{1} \mathrm{H}$ NMR (CDCl3, $\left.500 \mathrm{MHz}\right)$

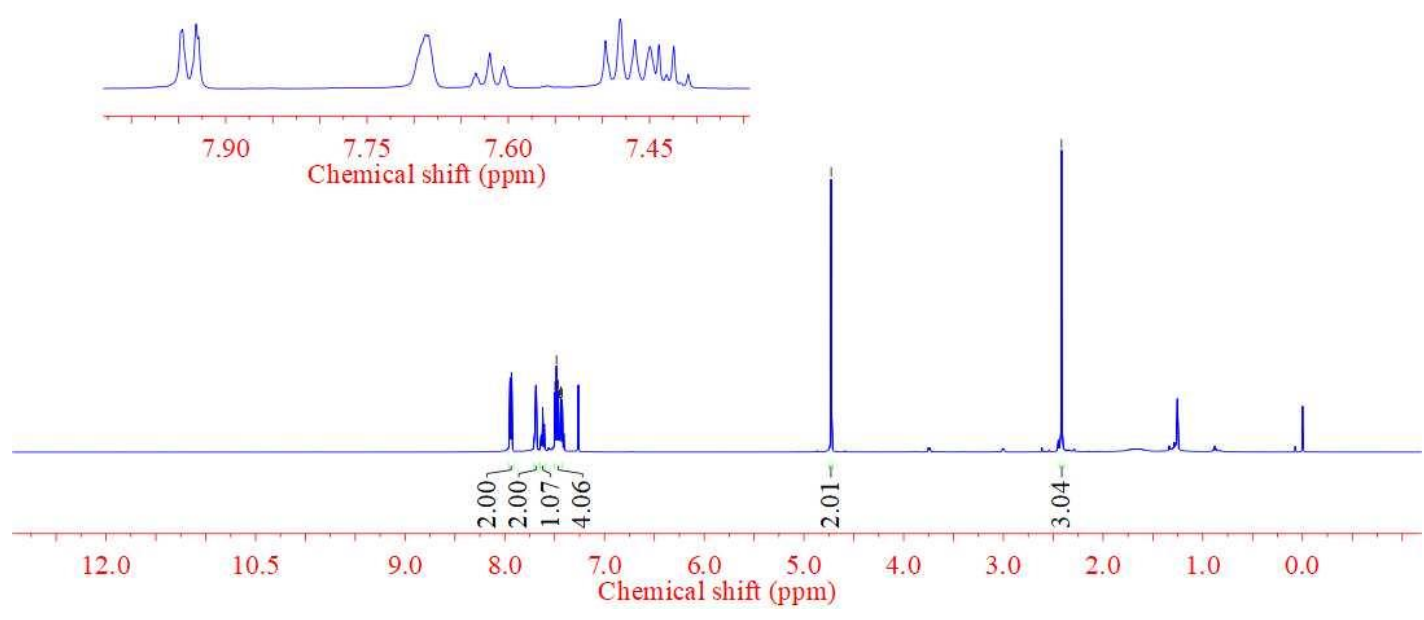

${ }^{1} \mathrm{H}$ NMR Spectrum of Compound 3ae

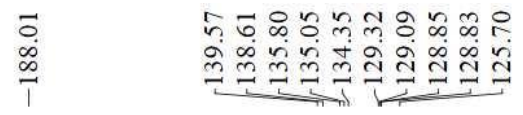

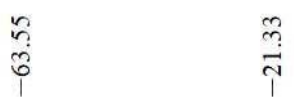<smiles>Cc1cccc(S(=O)(=O)CC(=O)c2ccccc2)c1</smiles>

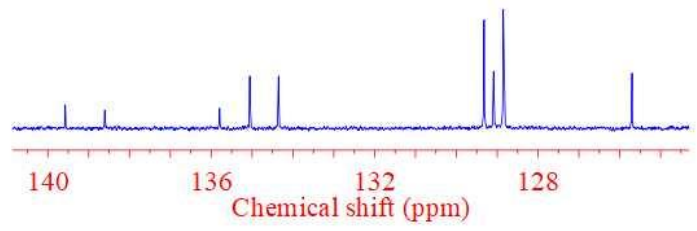

${ }^{13} \mathrm{C}\left\{{ }^{1} \mathrm{H}\right\} \mathrm{NMR}\left(\mathrm{CDCl}_{3}, 126 \mathrm{MHz}\right)$

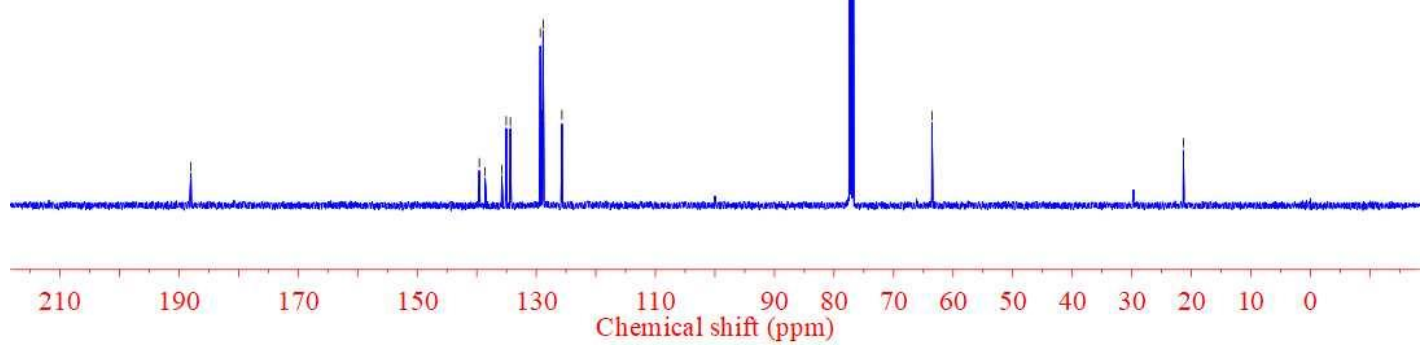

${ }^{13} \mathrm{C}\left\{{ }^{1} \mathrm{H}\right\}$ NMR Spectrum of Compound 3ae 
<smiles>O=C(CS(=O)(=O)c1cccc(F)c1)c1ccccc1</smiles>

${ }^{1} \mathrm{H}$ NMR $\left(\mathrm{CDCl}_{3}, 500 \mathrm{MHz}\right)$
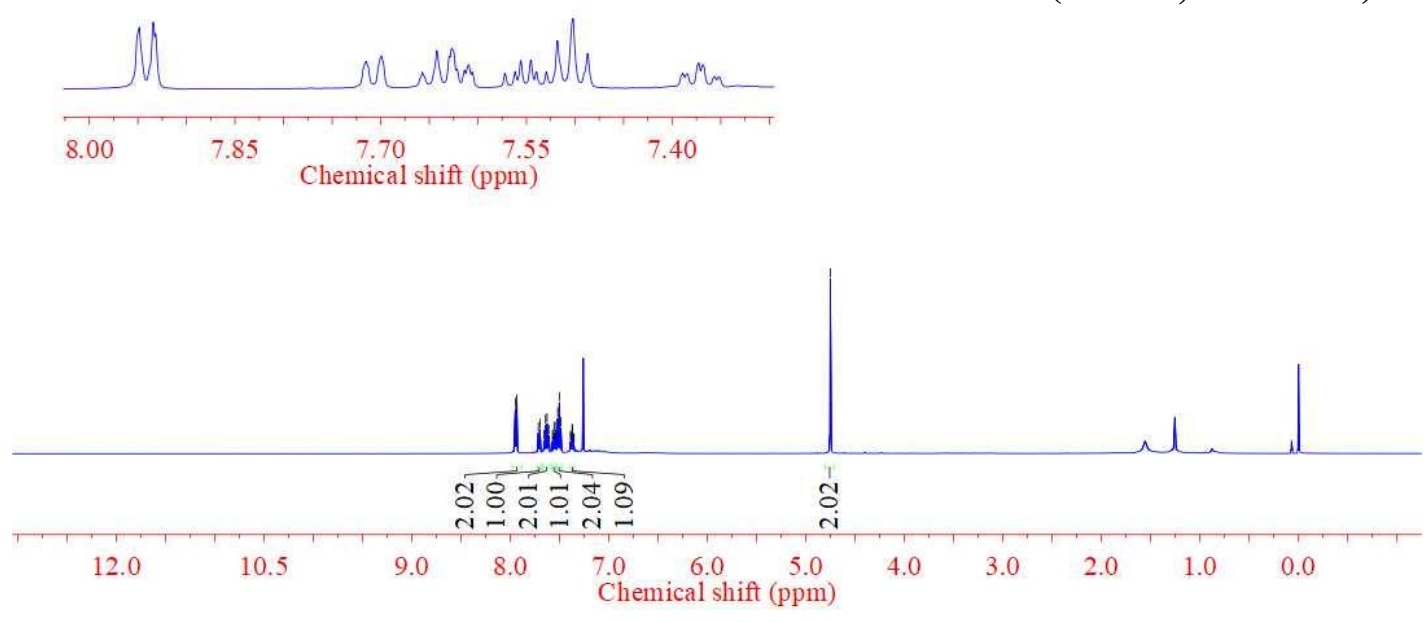

\section{${ }^{1}$ H NMR Spectrum of Compound 3af}
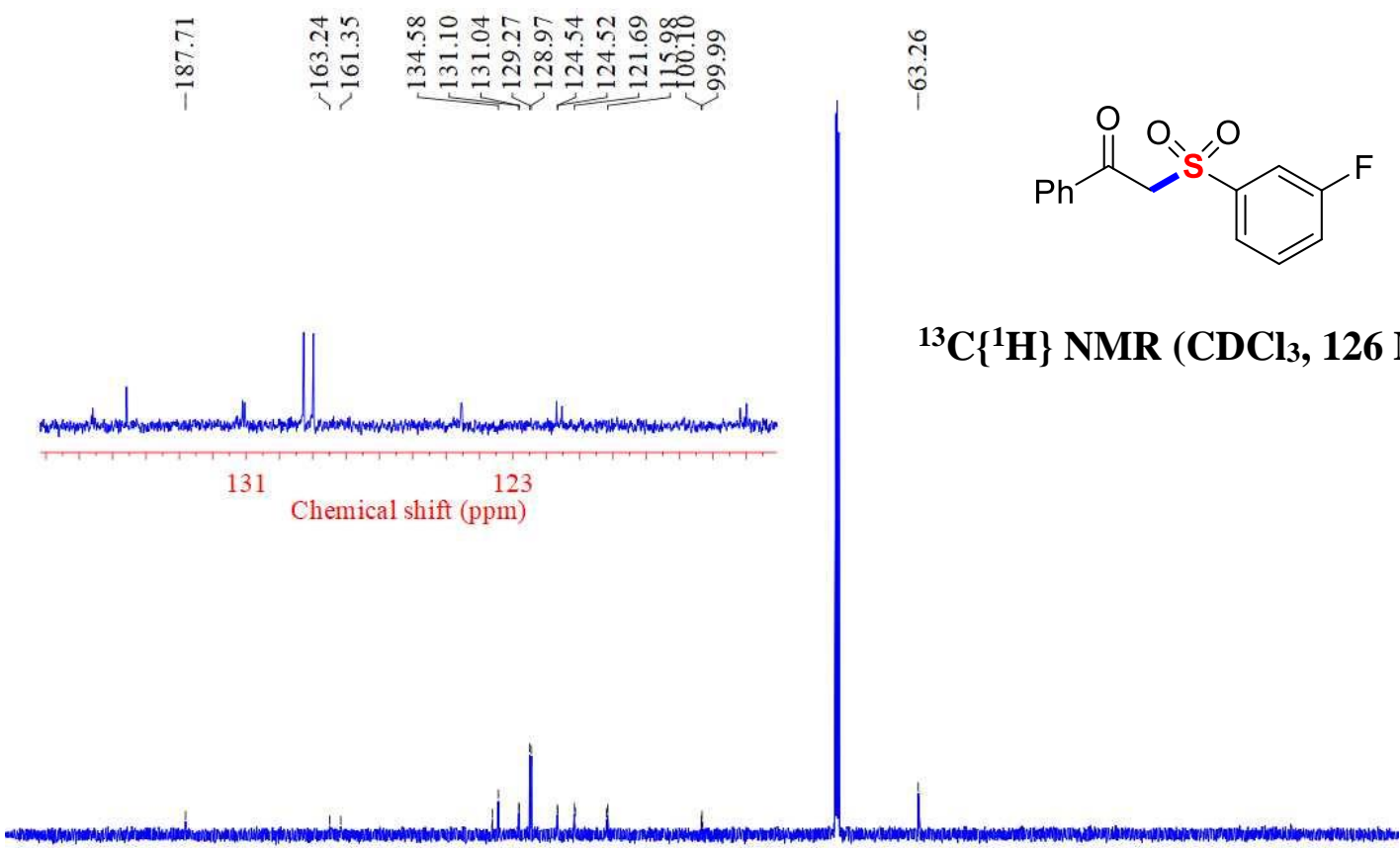

ָై<smiles>O=C(CS(=O)(=O)c1cccc(F)c1)c1ccccc1</smiles>

${ }^{13} \mathrm{C}\left\{{ }^{1} \mathrm{H}\right\} \mathrm{NMR}\left(\mathrm{CDCl}_{3}, 126 \mathrm{MHz}\right)$

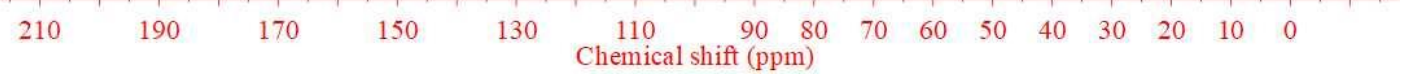

${ }^{13} \mathbf{C}\left\{{ }^{1} \mathbf{H}\right\}$ NMR Spectrum of Compound 3af 
$\infty$
$\infty$
$\infty$
0
1<smiles>O=C(CS(=O)(=O)c1cccc(F)c1)c1ccccc1</smiles>

${ }^{19} \mathrm{~F}\left\{{ }^{1} \mathrm{H}\right\}$ NMR $\left(\mathrm{CDCl}_{3}, 471 \mathrm{MHz}\right)$

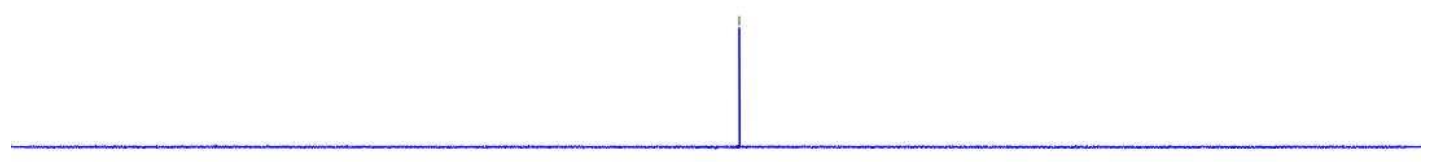

$\begin{array}{lllllllllll}10 & 0 & -10 & -30 & -50 & -70 & -90 & -110 \\ \text { Chemical shift }(\mathrm{ppm}) & -130 & -150 & -170 & -190 & -210\end{array}$

${ }^{19} \mathbf{F}\left\{{ }^{1} \mathbf{H}\right\}$ NMR Spectrum of Compound 3af

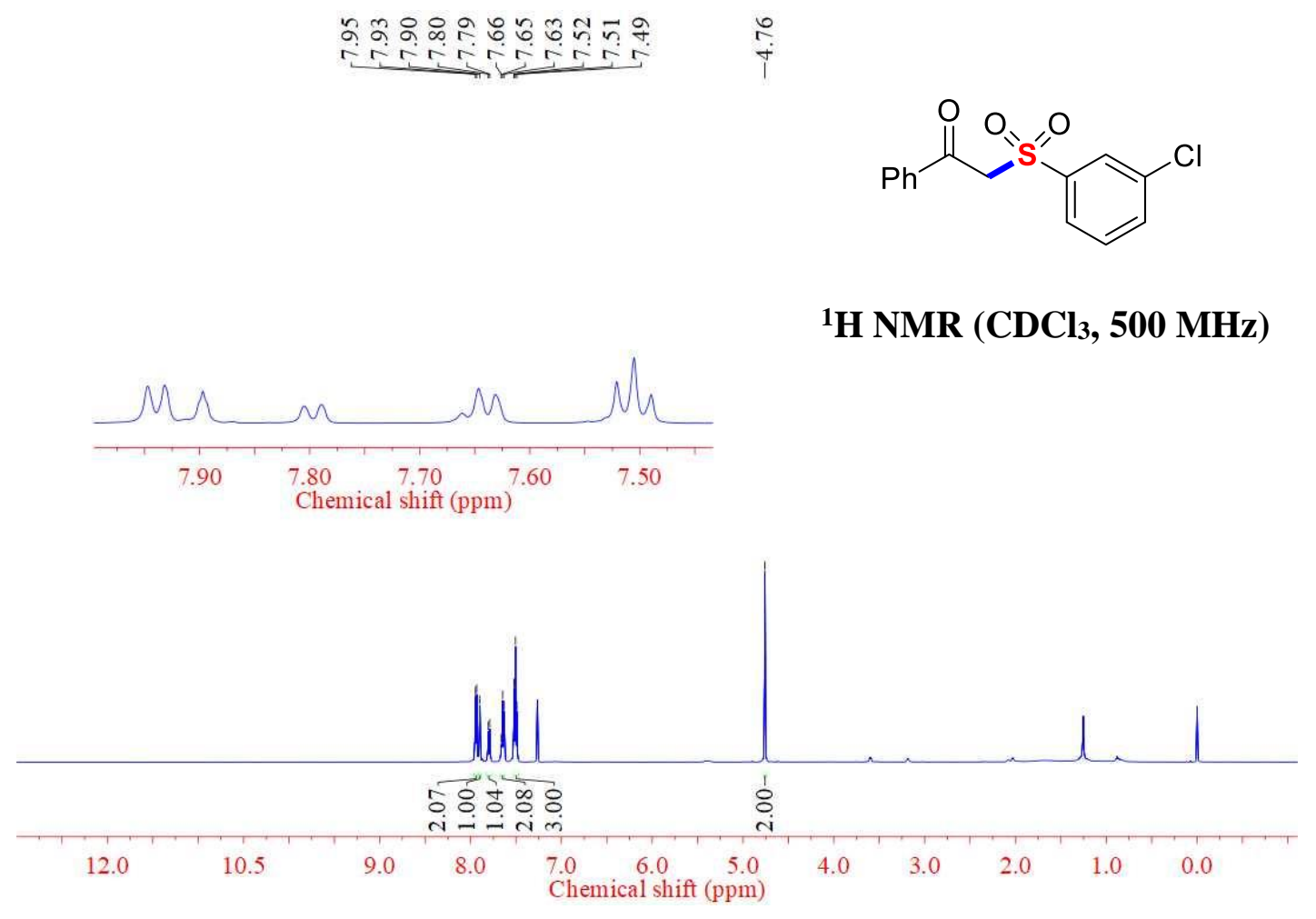

${ }^{1} \mathrm{H}$ NMR Spectrum of Compound 3ag 


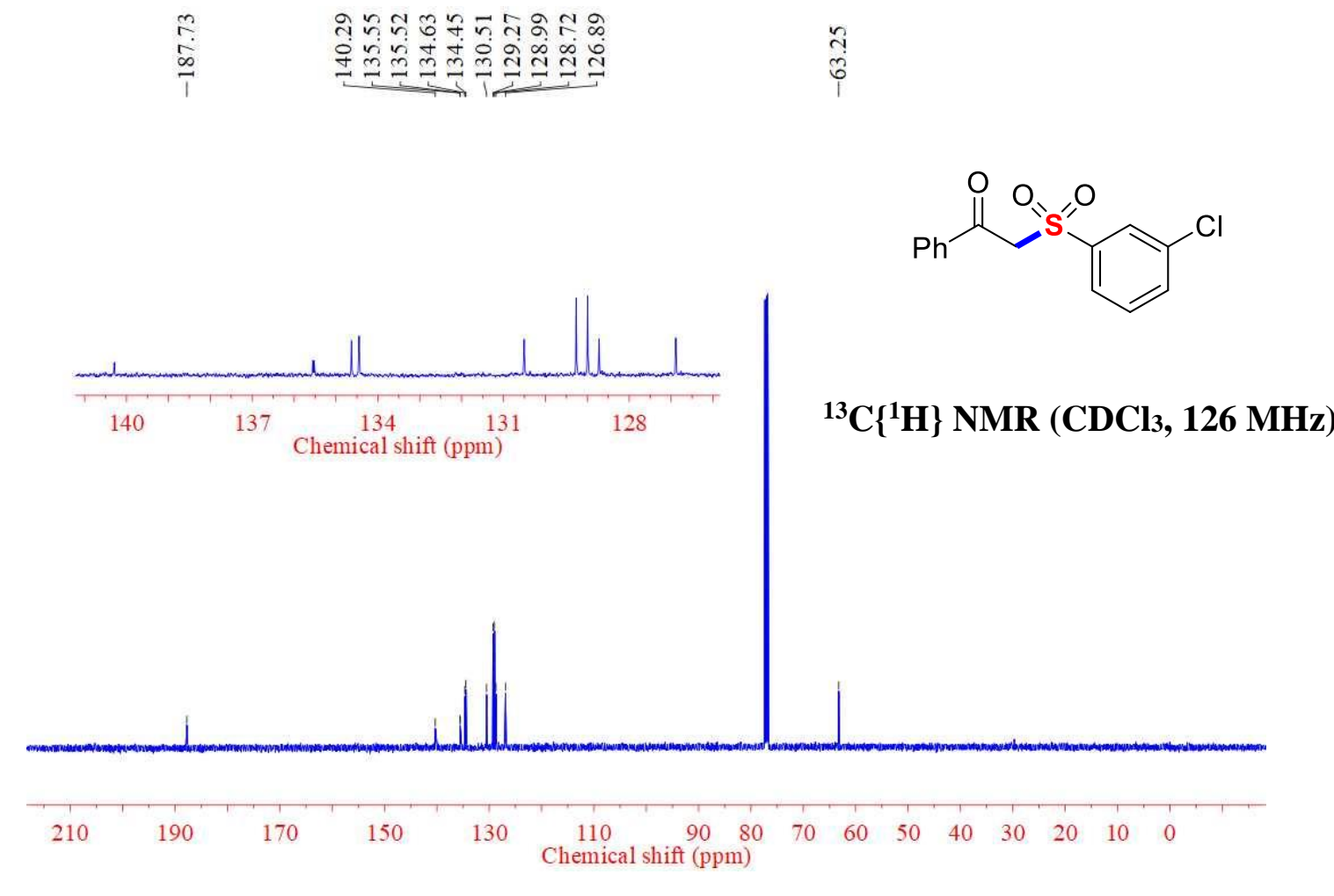

${ }^{13} \mathbf{C}\left\{{ }^{1} \mathbf{H}\right\}$ NMR Spectrum of Compound 3ag<smiles>O=C(CS(=O)(=O)c1cccc(Br)c1)c1ccccc1</smiles>

${ }^{1} \mathrm{H}$ NMR (CDCl3, $\left.500 \mathrm{MHz}\right)$
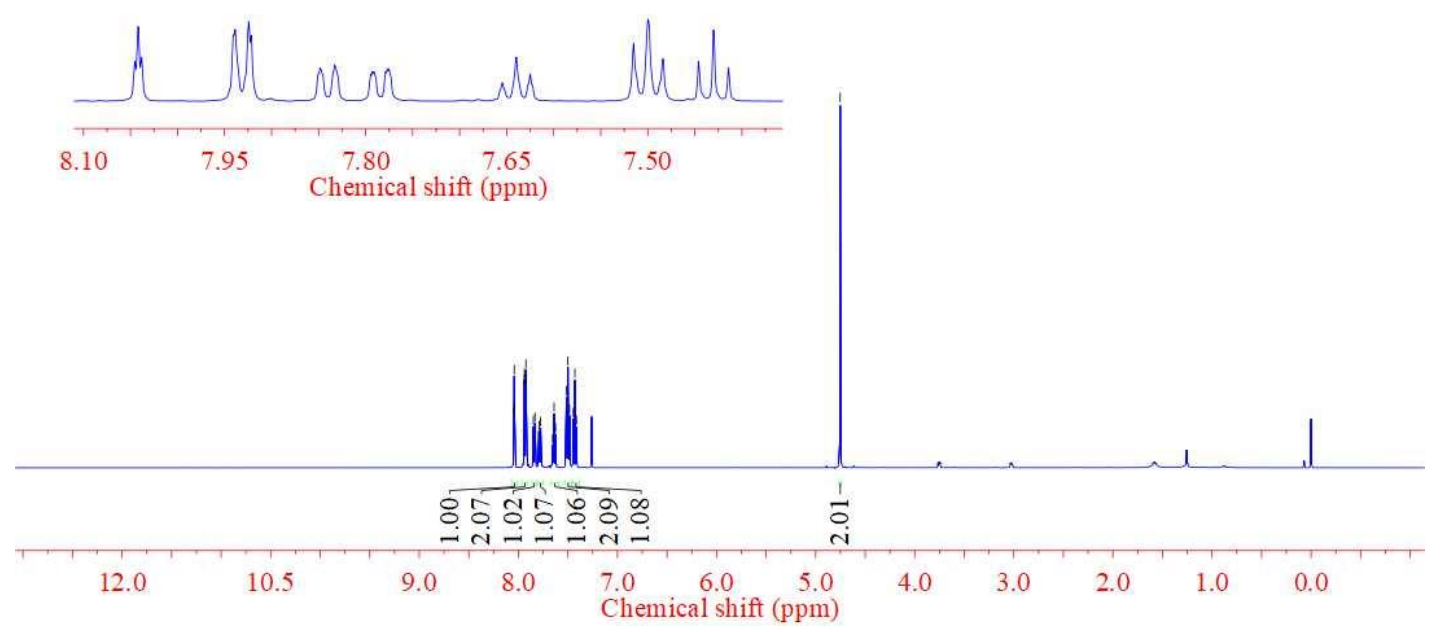

${ }^{1} \mathrm{H}$ NMR Spectrum of Compound 3ah 


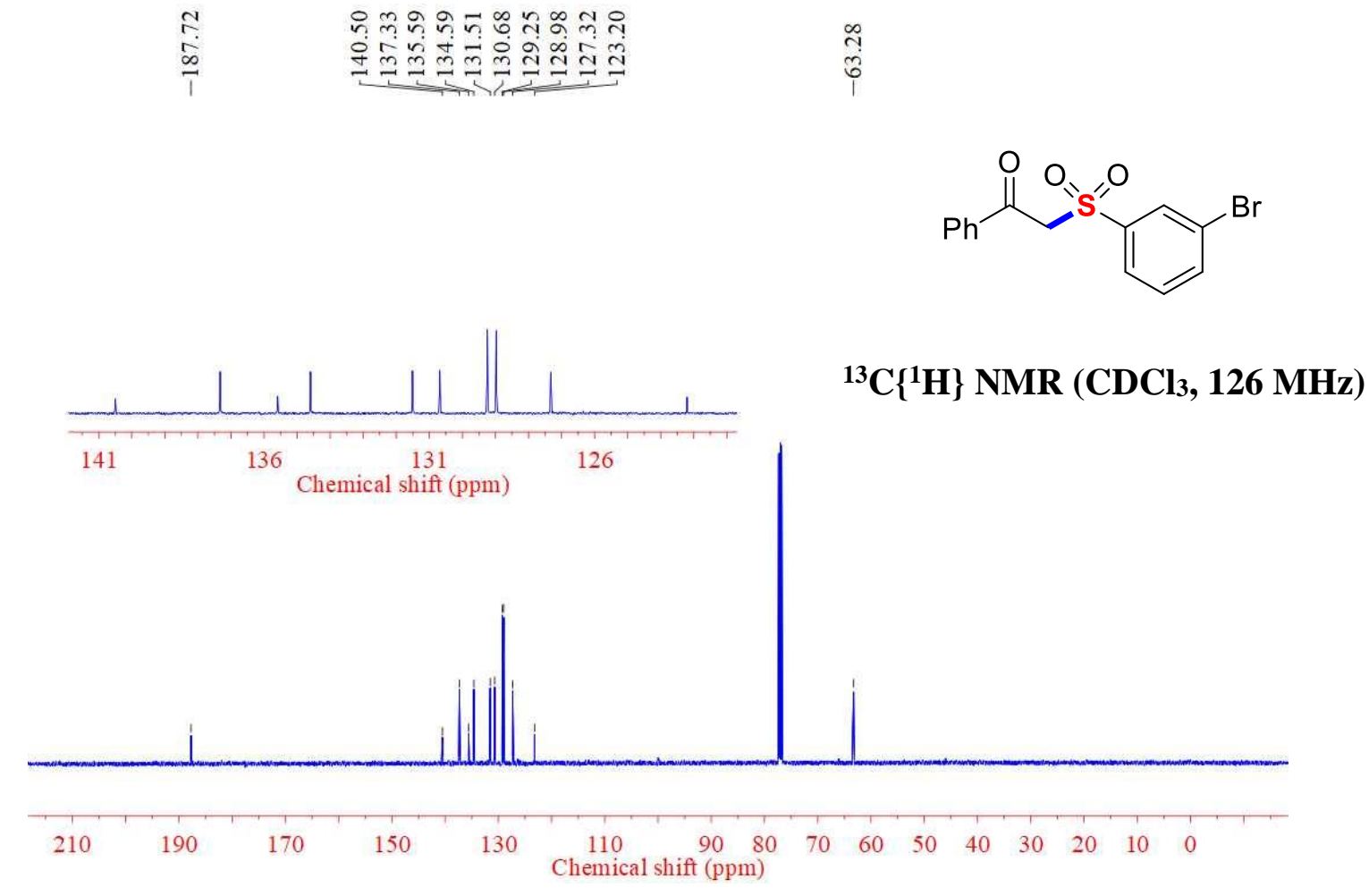

${ }^{13} \mathbf{C}\left\{{ }^{1} \mathbf{H}\right\}$ NMR Spectrum of Compound 3ah

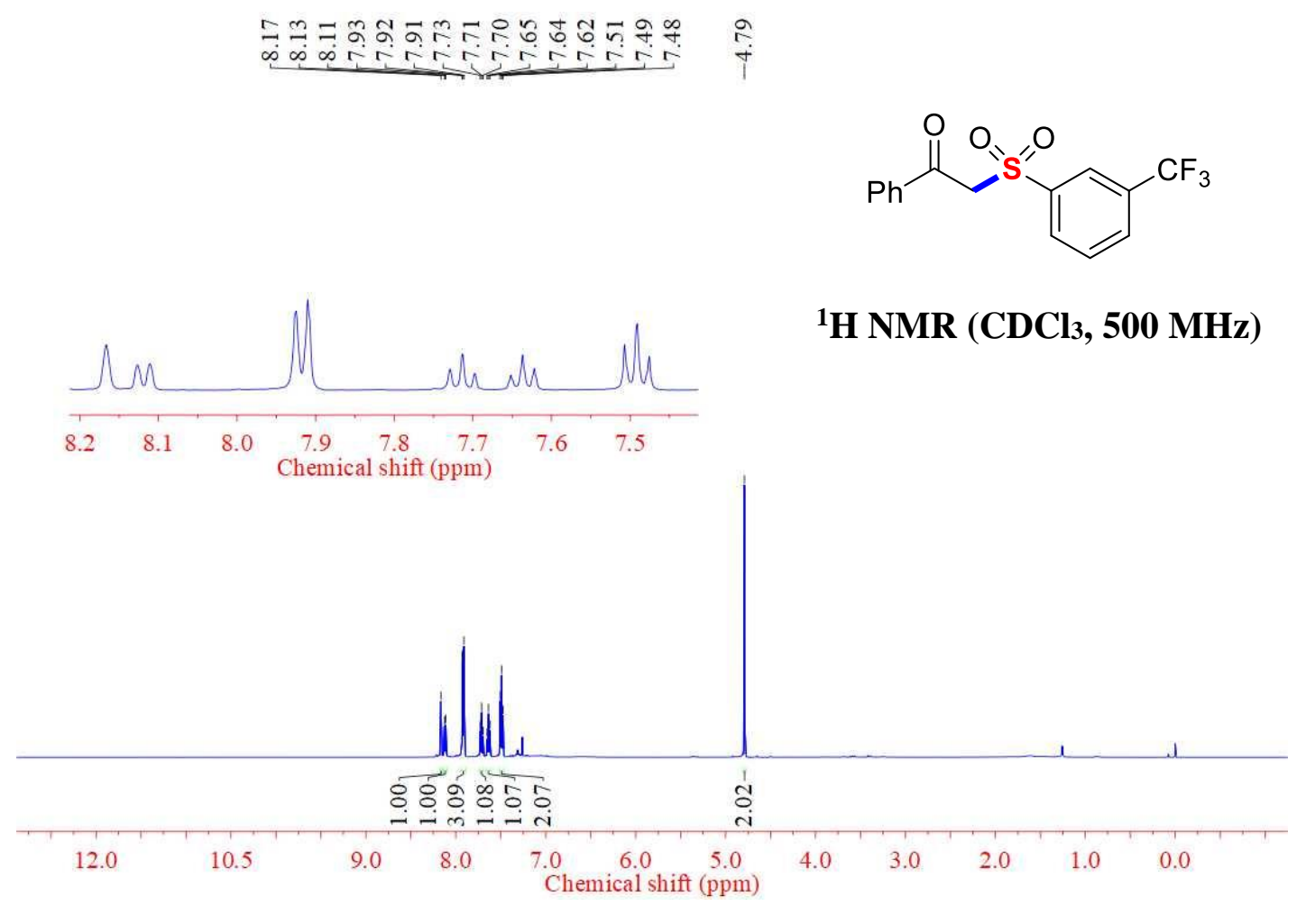

${ }^{1} \mathrm{H}$ NMR Spectrum of Compound 3ai 


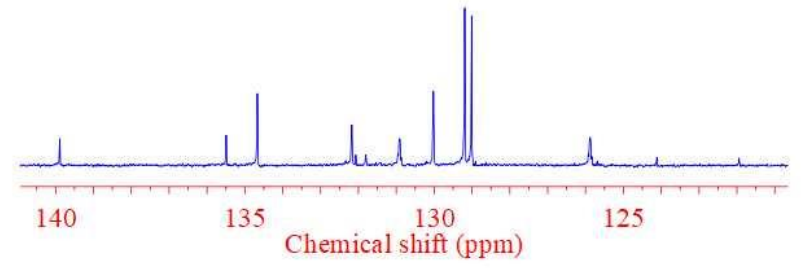<smiles>O=C(CS(=O)(=O)c1cccc(C(F)(F)F)c1)c1ccccc1</smiles>

${ }^{13} \mathrm{C}\left\{{ }^{1} \mathrm{H}\right\} \mathrm{NMR}\left(\mathrm{CDCl}_{3}, 126 \mathrm{MHz}\right)$

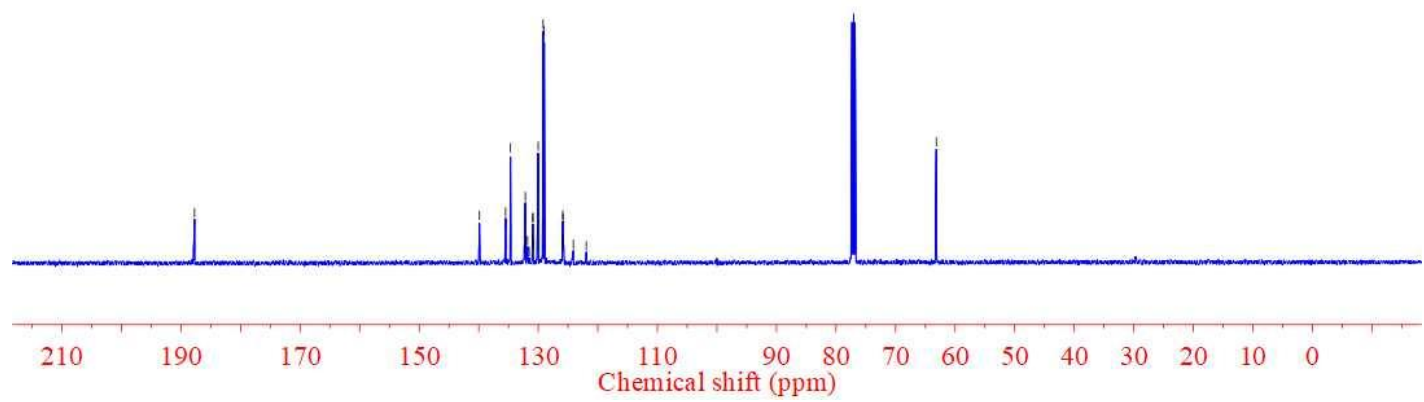

${ }^{13} \mathrm{C}\left\{{ }^{1} \mathbf{H}\right\}$ NMR Spectrum of Compound 3ai

$\infty$
$\infty$
ci
$i$<smiles>O=C(CS(=O)(=O)c1cccc(C(F)(F)F)c1)c1ccccc1</smiles>

${ }^{19} \mathrm{~F}\left\{{ }^{1} \mathrm{H}\right\}$ NMR $\left(\mathrm{CDCl}_{3}, 471 \mathrm{MHz}\right)$

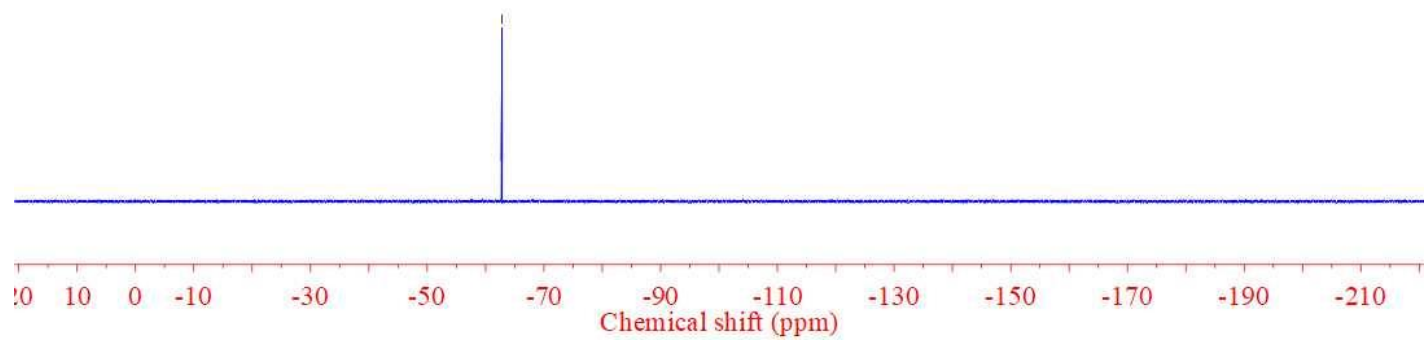

${ }^{13} \mathrm{C}\left\{{ }^{1} \mathrm{H}\right\}$ NMR Spectrum of Compound 3ai 
Analysis Info

Analysis Name

Method

Sample Name

Comment
Acquisition Date $\quad 8 / 17 / 2020$ 7:54:44 PM

D:IDatalQ-TOF-ZHANGPF-200817-hl-3aj_01.d

ESI+100-750-200615.m

Q-TOF-XULW-200817-xjl_01

Operator BDAL@DE

Instrument/Ser\# microTOF-Q II 228888.10

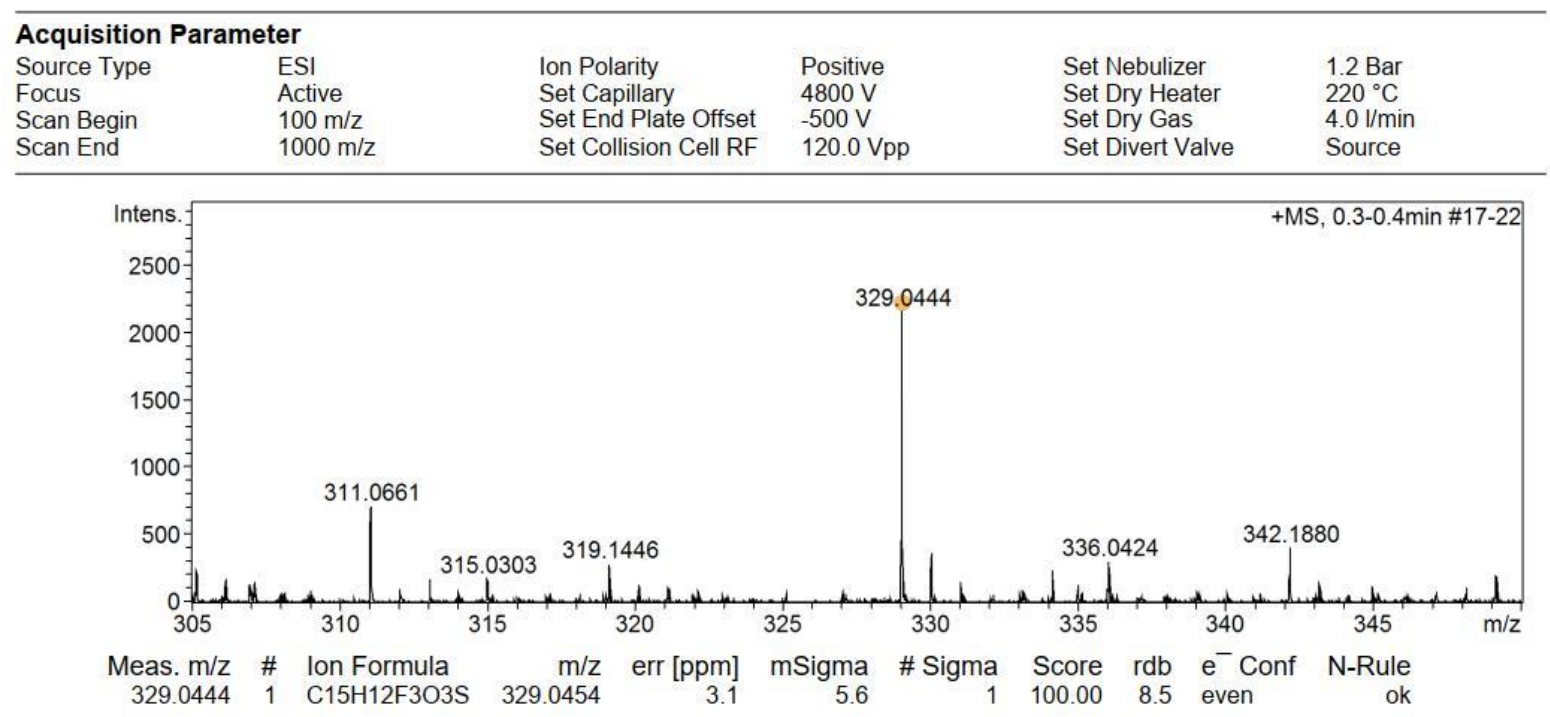

HRMS Spectrum of Compound 3ai

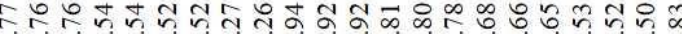

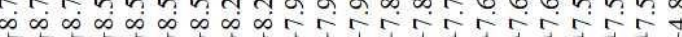<smiles>O=C(CS(=O)(=O)c1cccc([N+](=O)[O-])c1)c1ccccc1</smiles>

${ }^{1} \mathrm{H}$ NMR (CDCl3, $\left.500 \mathrm{MHz}\right)$
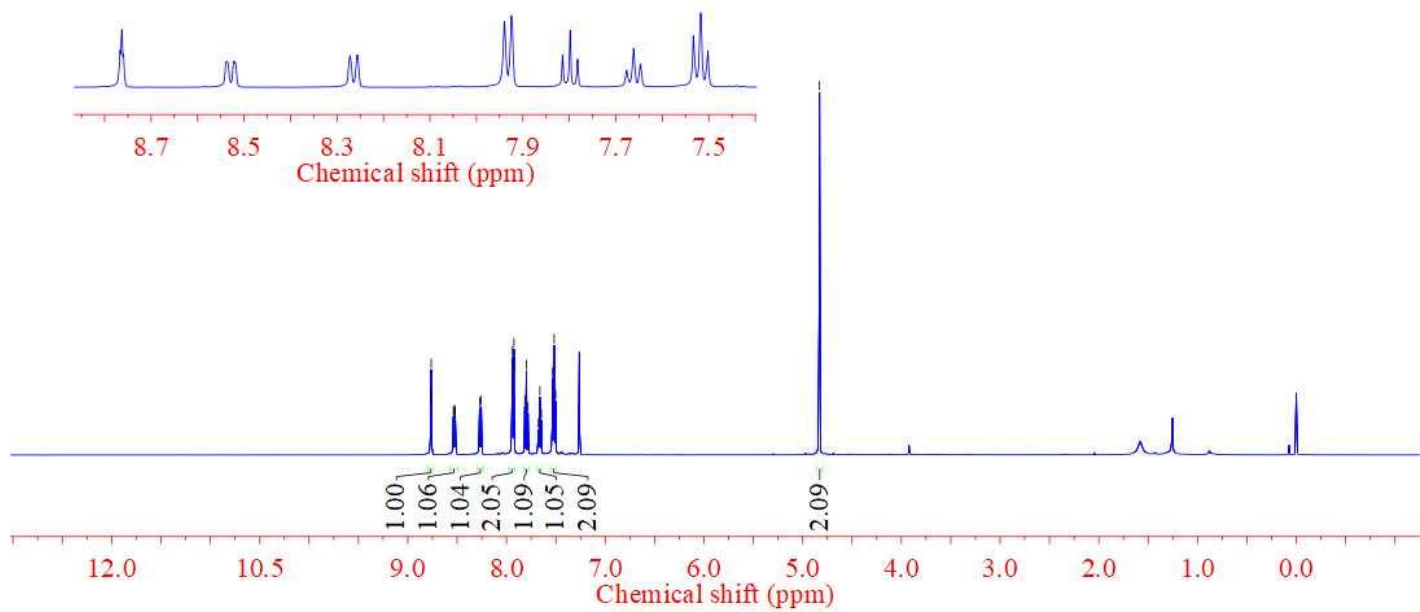

${ }^{1}$ H NMR Spectrum of Compound 3aj 


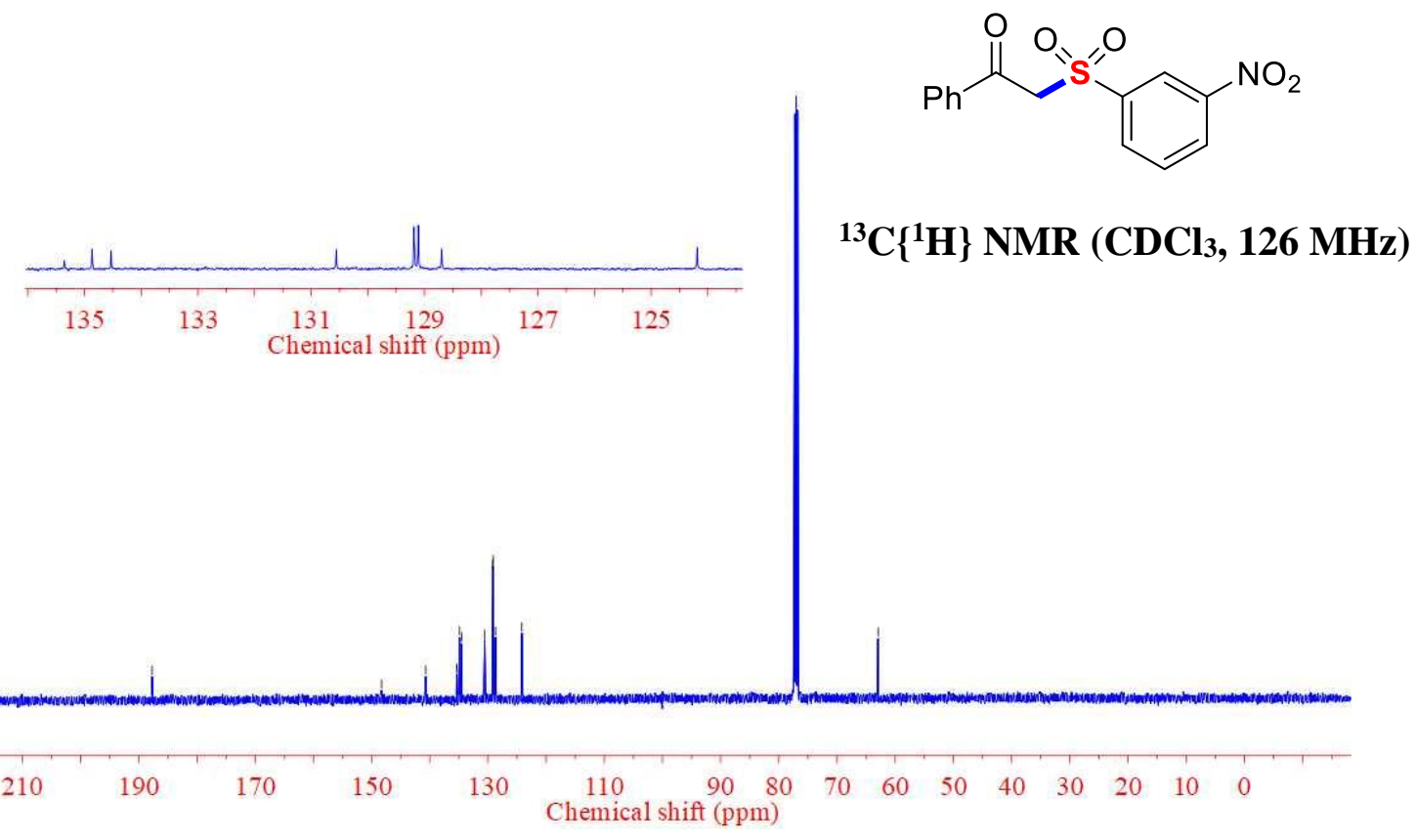

\section{${ }^{13} \mathrm{C}\left\{{ }^{1} \mathrm{H}\right\}$ NMR Spectrum of Compound 3aj}

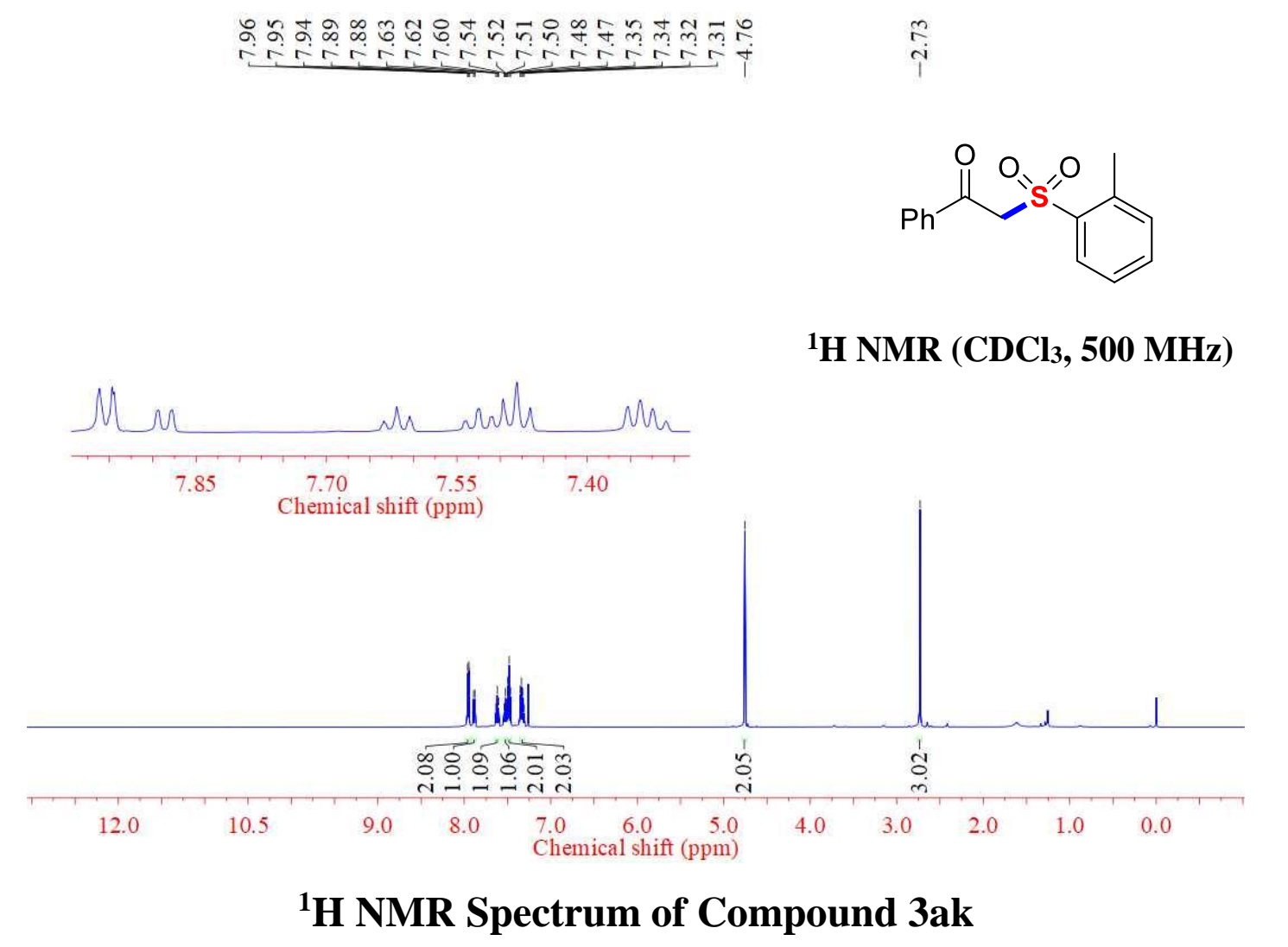




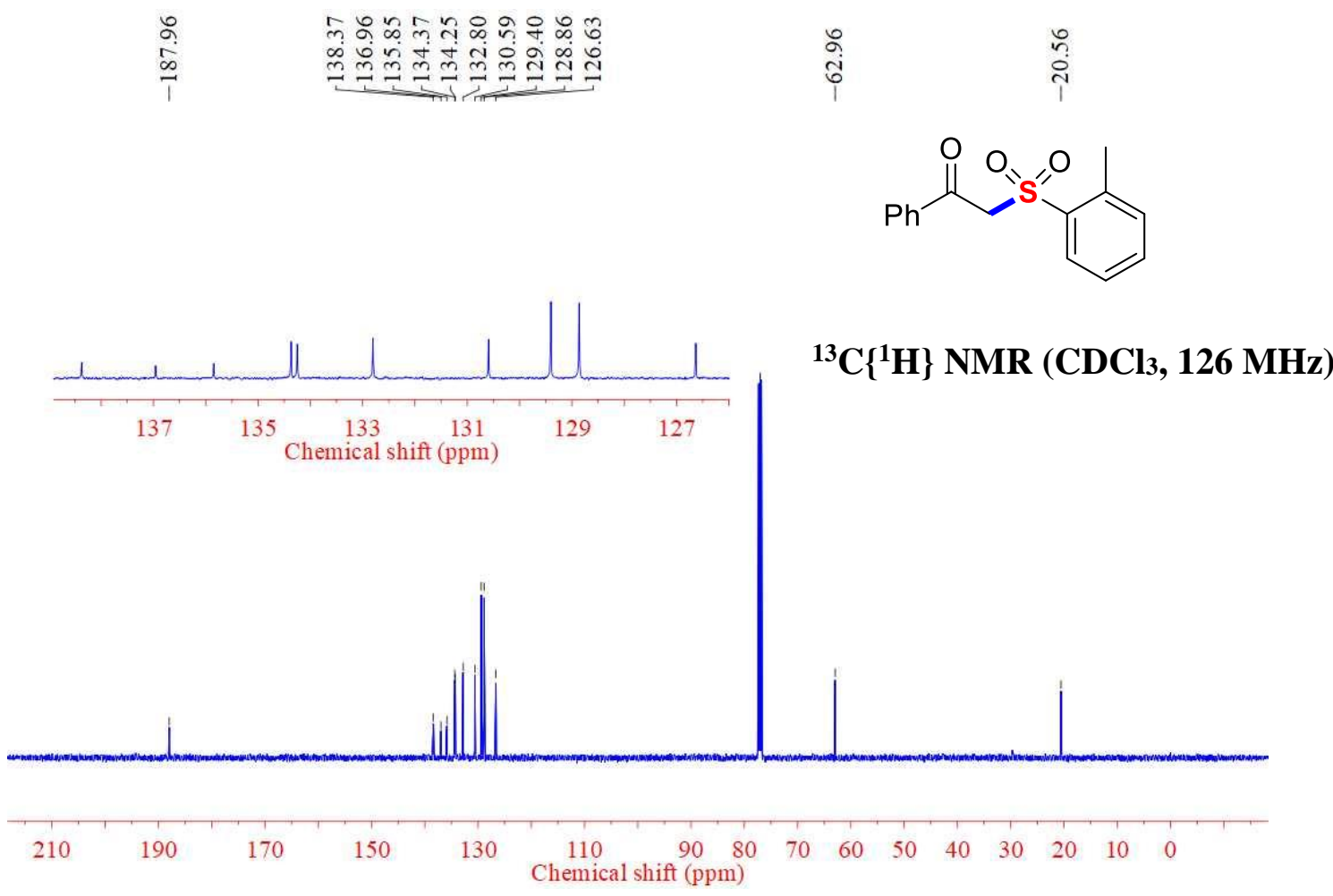

${ }^{13} \mathrm{C}\left\{{ }^{1} \mathbf{H}\right\}$ NMR Spectrum of Compound 3ak

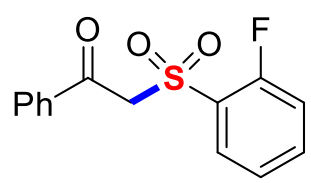

${ }^{1} \mathrm{H}$ NMR $\left(\mathrm{CDCl}_{3}, 500 \mathrm{MHz}\right)$
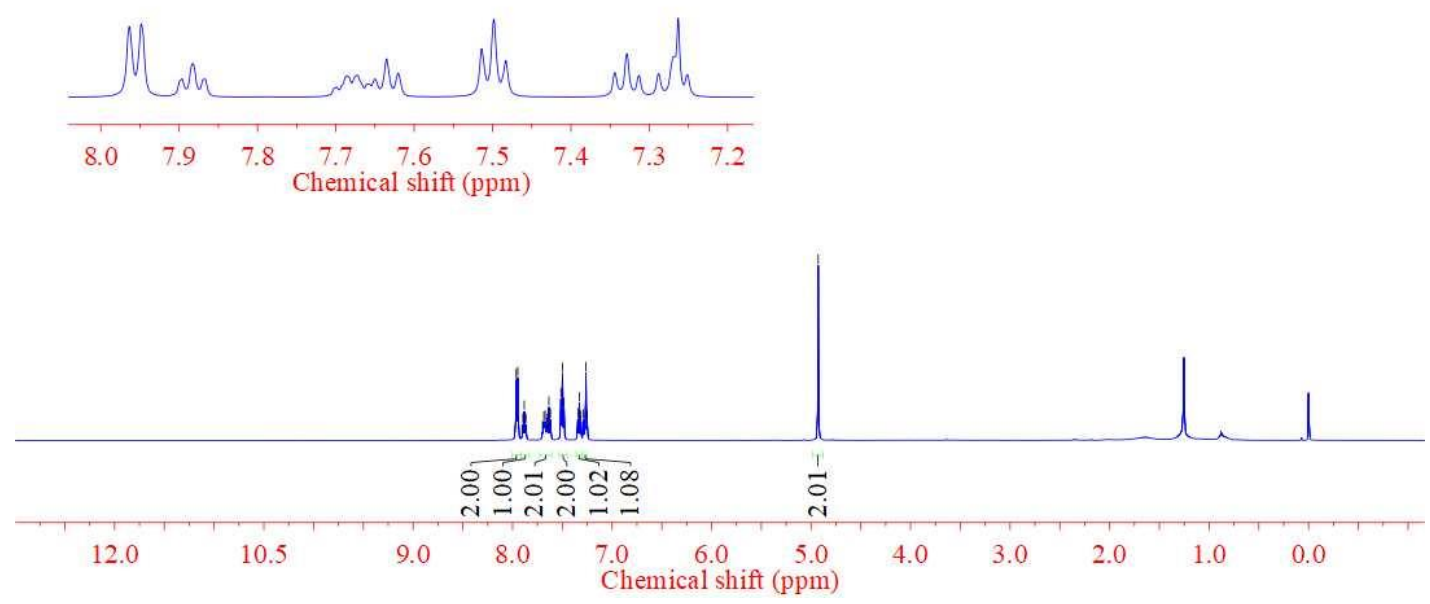

${ }^{1} \mathrm{H}$ NMR Spectrum of Compound 3al 

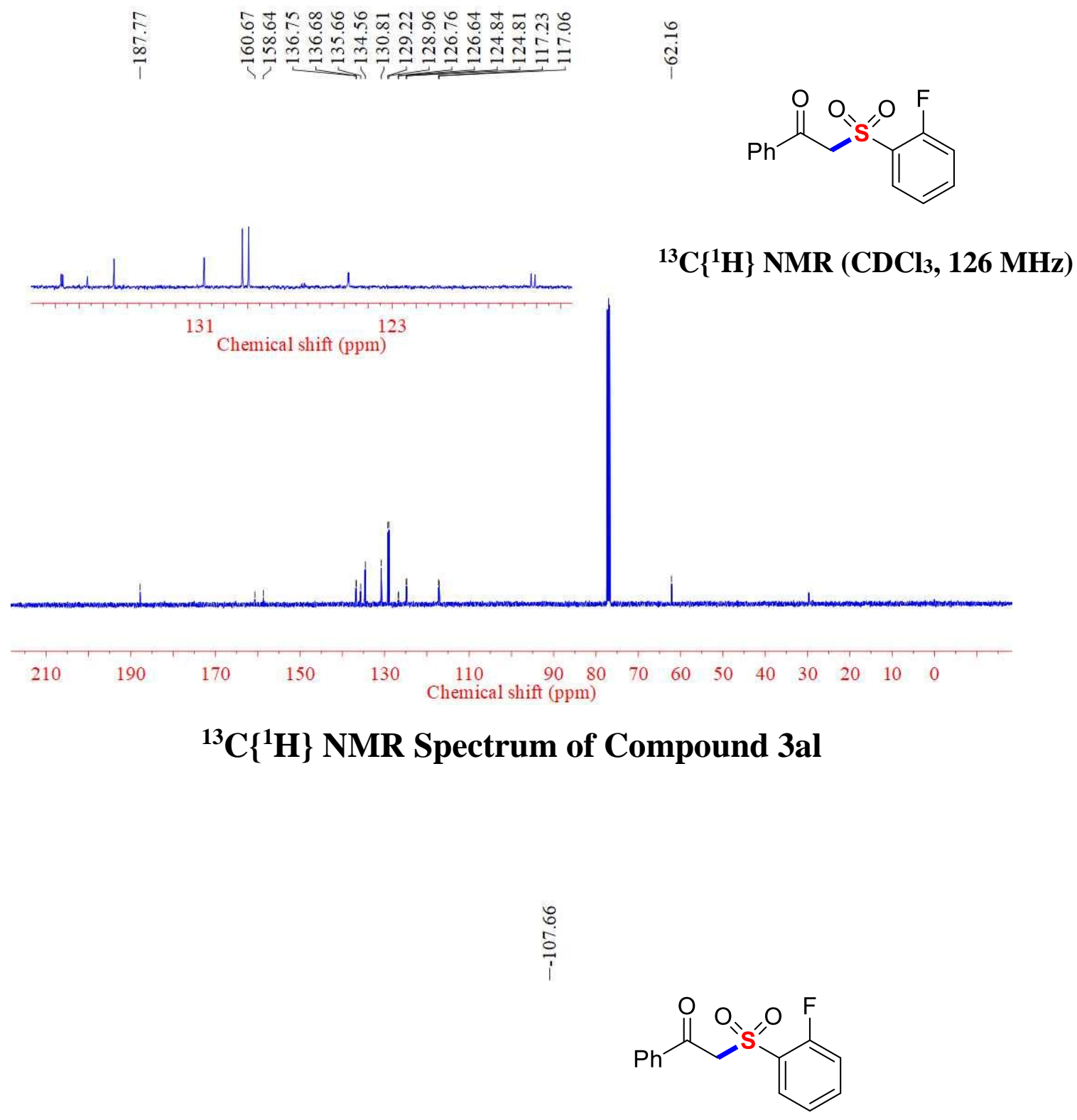

${ }^{19} \mathrm{~F}\left\{{ }^{1} \mathrm{H}\right\}$ NMR $\left(\mathrm{CDCl}_{3}, 471 \mathrm{MHz}\right)$

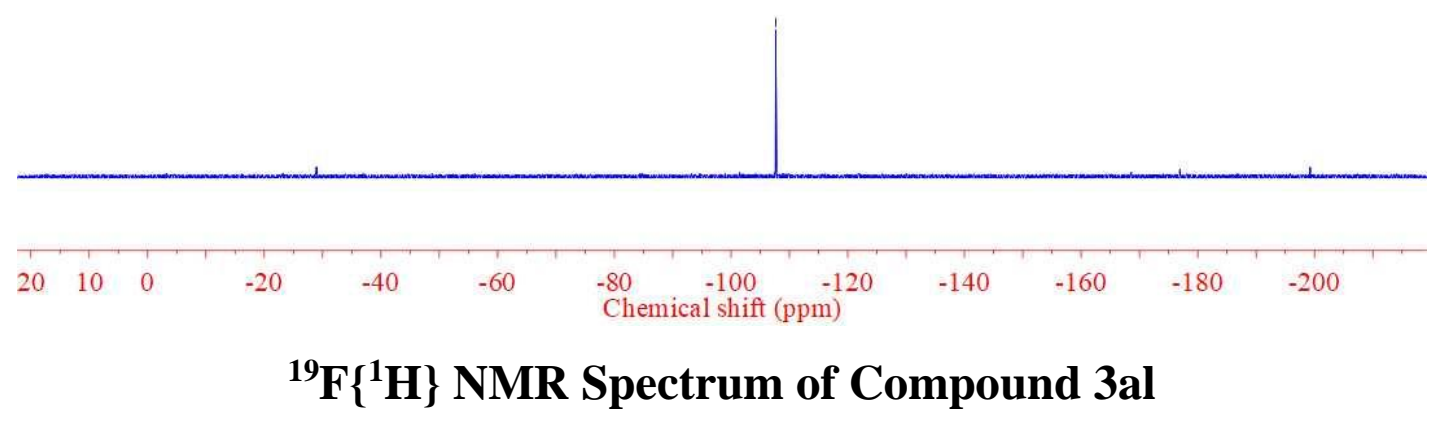


<smiles>O=C(CS(=O)(=O)c1ccccc1Br)c1ccccc1</smiles>

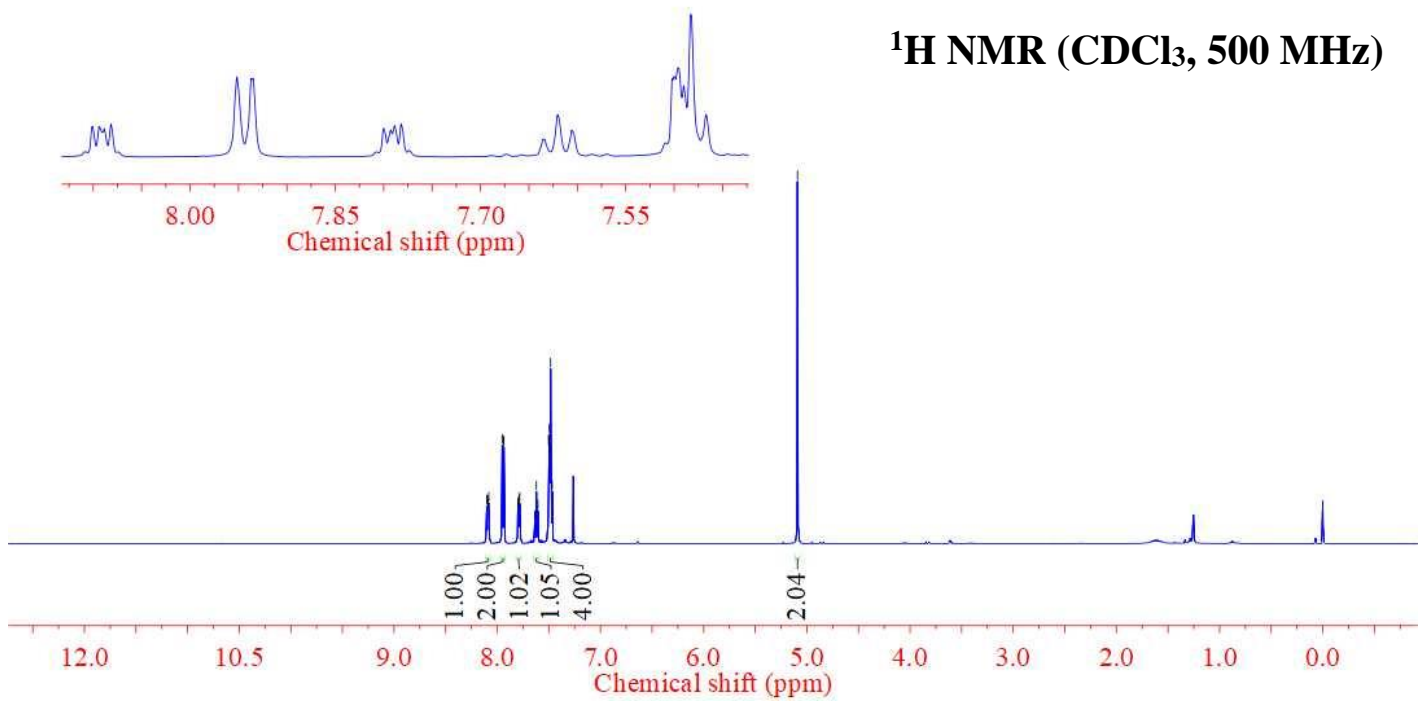

${ }^{1}$ H NMR Spectrum of Compound 3am

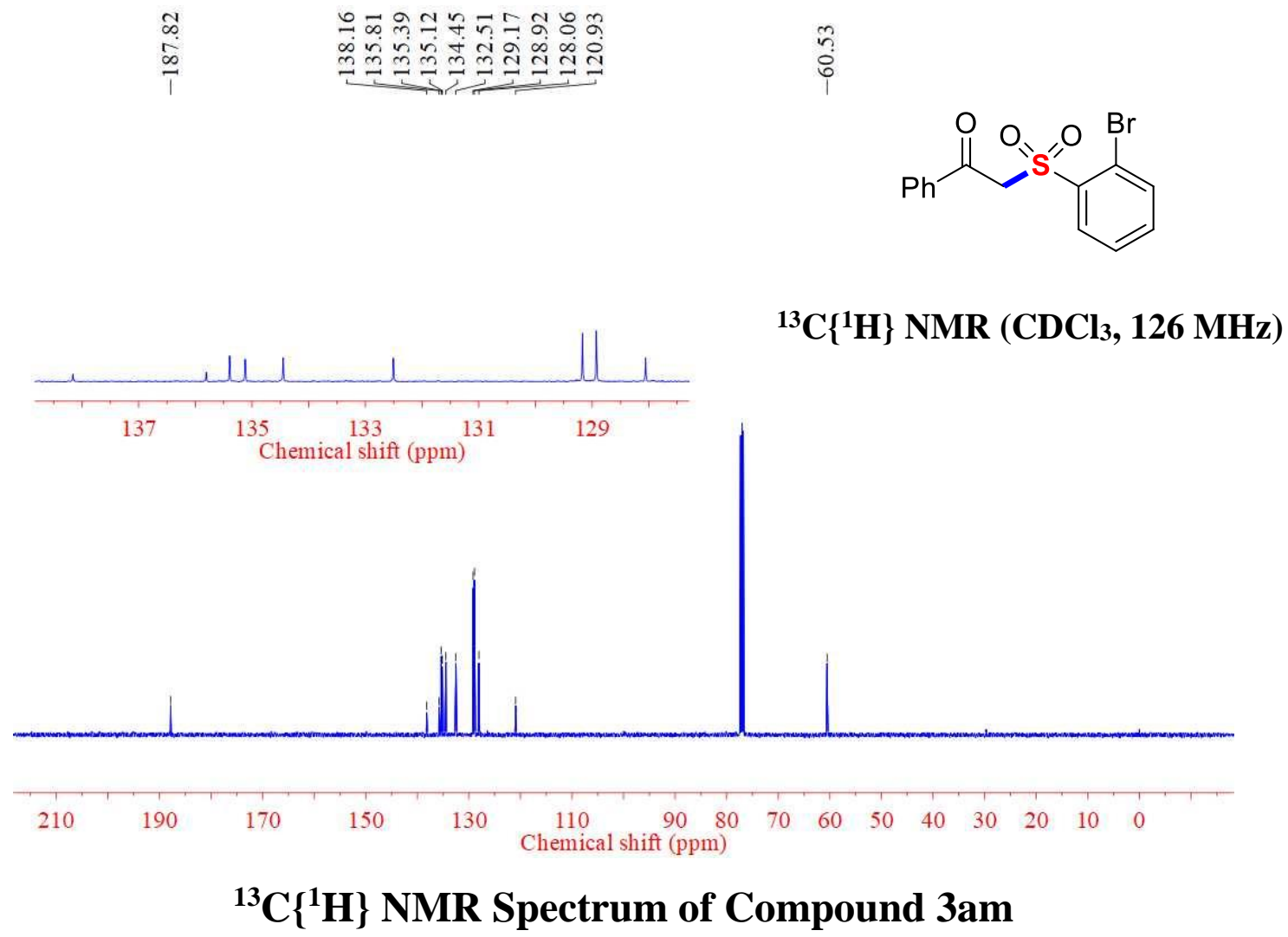


<smiles>O=C(CS(=O)(=O)c1ccccc1C(F)(F)F)c1ccccc1</smiles>

${ }^{1} \mathrm{H}$ NMR (CDCl3, $\left.500 \mathrm{MHz}\right)$
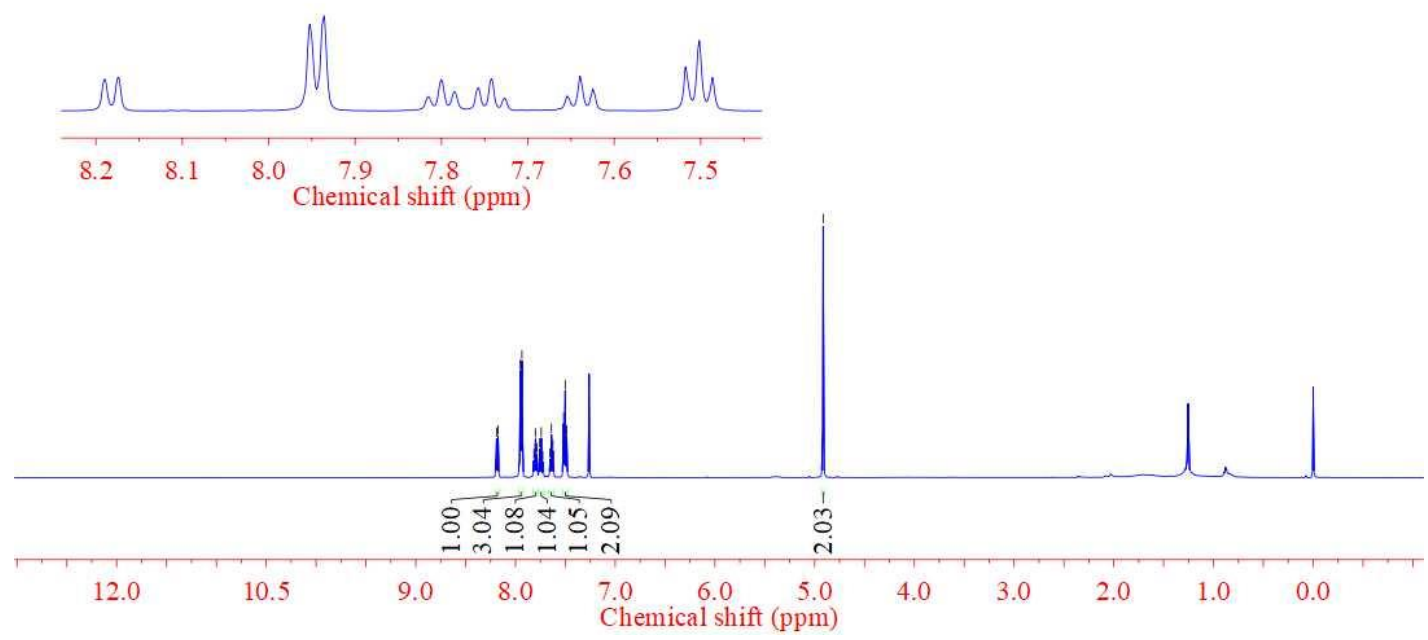

${ }^{1} \mathrm{H}$ NMR Spectrum of Compound 3an

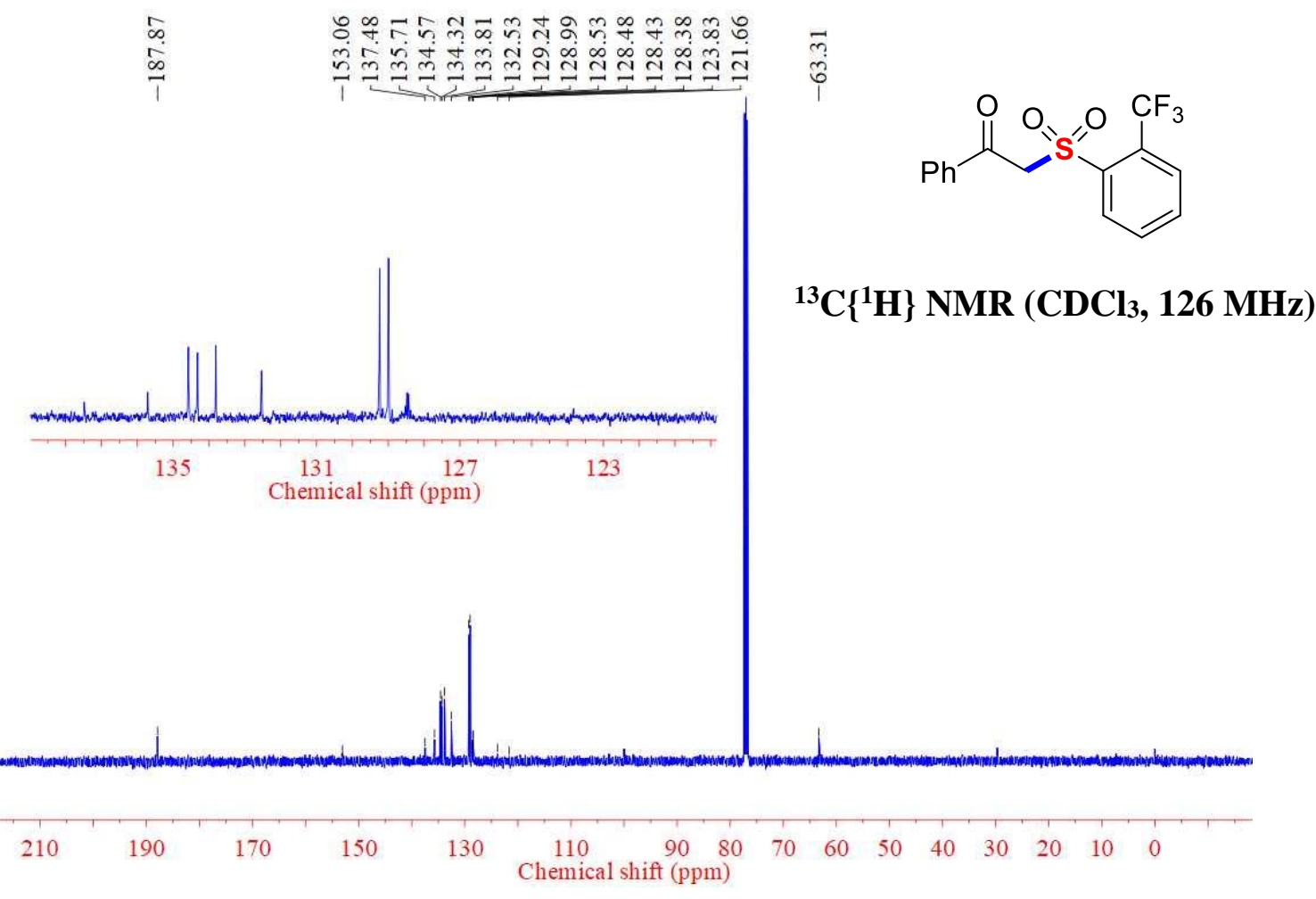

${ }^{13} \mathbf{C}\left\{{ }^{1} \mathbf{H}\right\}$ NMR Spectrum of Compound 3an 
<smiles>O=C(CS(=O)(=O)c1ccccc1C(F)(F)F)c1ccccc1</smiles>

${ }^{19} \mathrm{~F}\left\{{ }^{1} \mathrm{H}\right\}$ NMR $\left(\mathrm{CDCl}_{3}, 471 \mathrm{MHz}\right)$

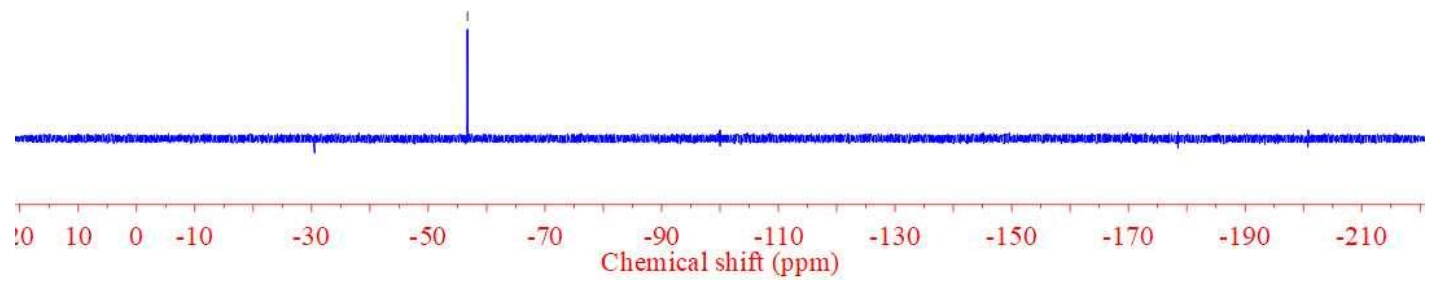

${ }^{19} \mathbf{F}\left\{{ }^{1} \mathbf{H}\right\}$ NMR Spectrum of Compound 3an

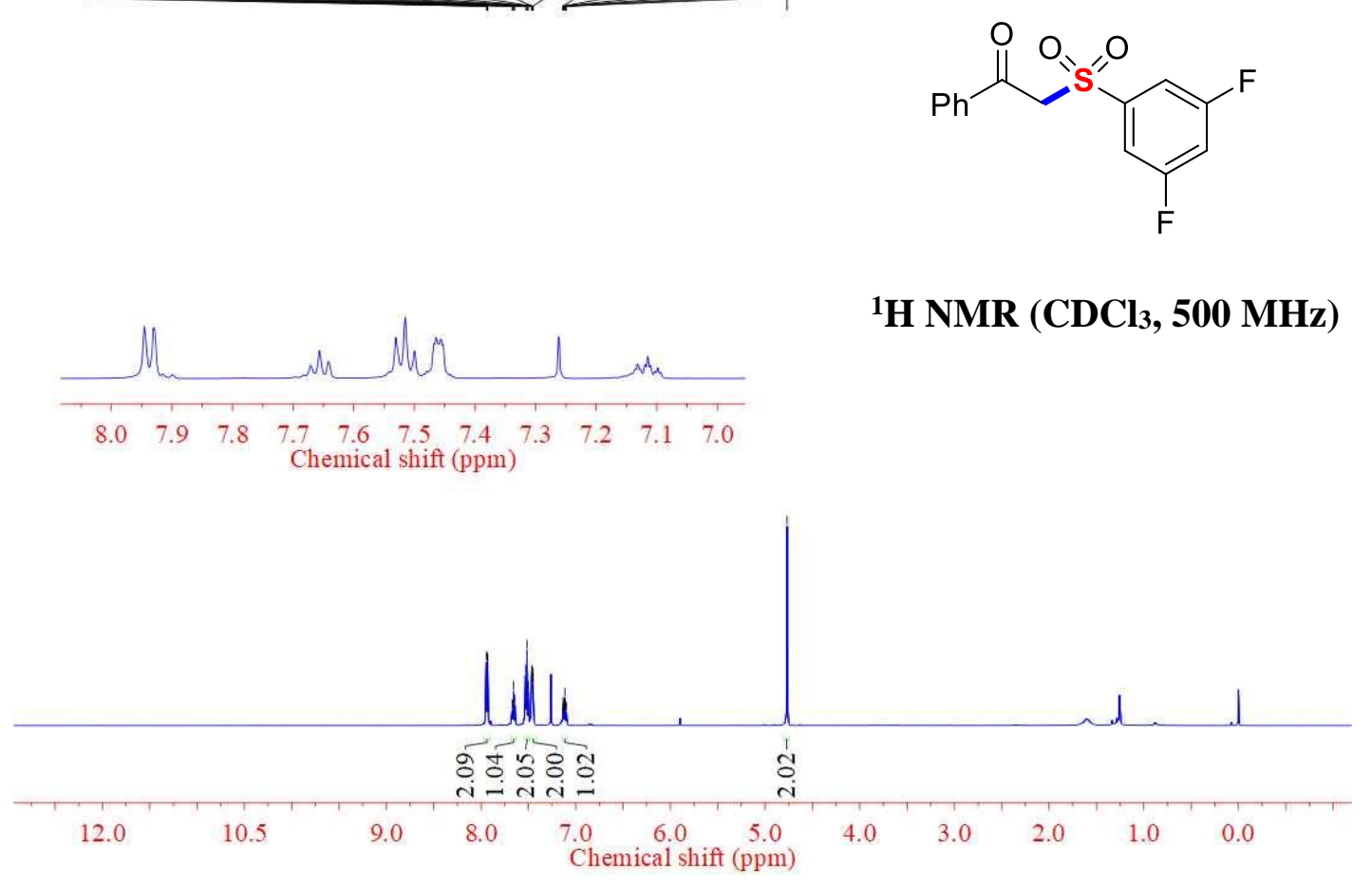

${ }^{1}$ H NMR Spectrum of Compound 3ao 
ڤે<smiles>O=C(CS(=O)(=O)c1cc(F)cc(F)c1)c1ccccc1</smiles>

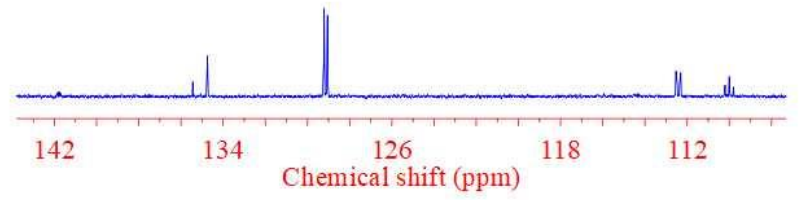

${ }^{13} \mathrm{C}\left\{{ }^{1} \mathrm{H}\right\} \mathrm{NMR}\left(\mathrm{CDCl}_{3}, 126 \mathrm{MHz}\right)$

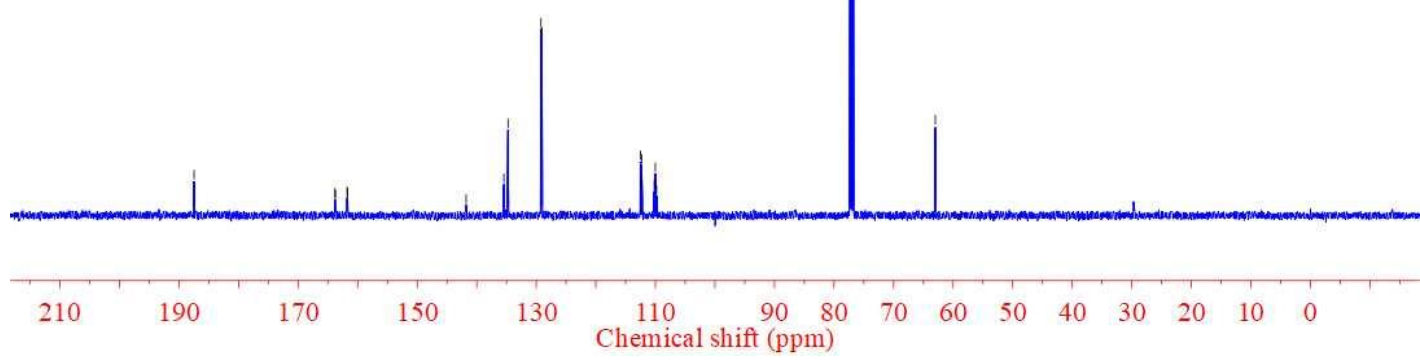

${ }^{13} \mathrm{C}\left\{{ }^{1} \mathrm{H}\right\}$ NMR Spectrum of Compound 3ao

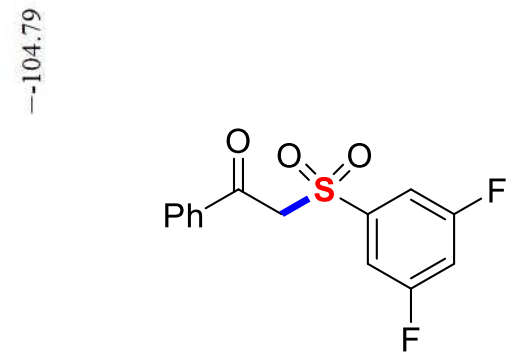

${ }^{19} \mathrm{~F}\left\{{ }^{1} \mathrm{H}\right\}$ NMR $\left(\mathrm{CDCl}_{3}, 471 \mathrm{MHz}\right)$

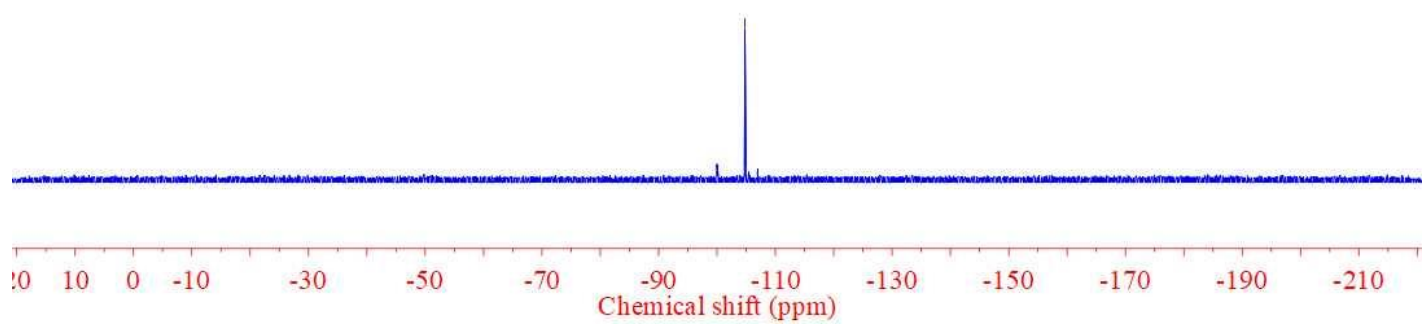

${ }^{19} \mathbf{F}\left\{{ }^{1} \mathrm{H}\right\}$ NMR Spectrum of Compound 3ao 


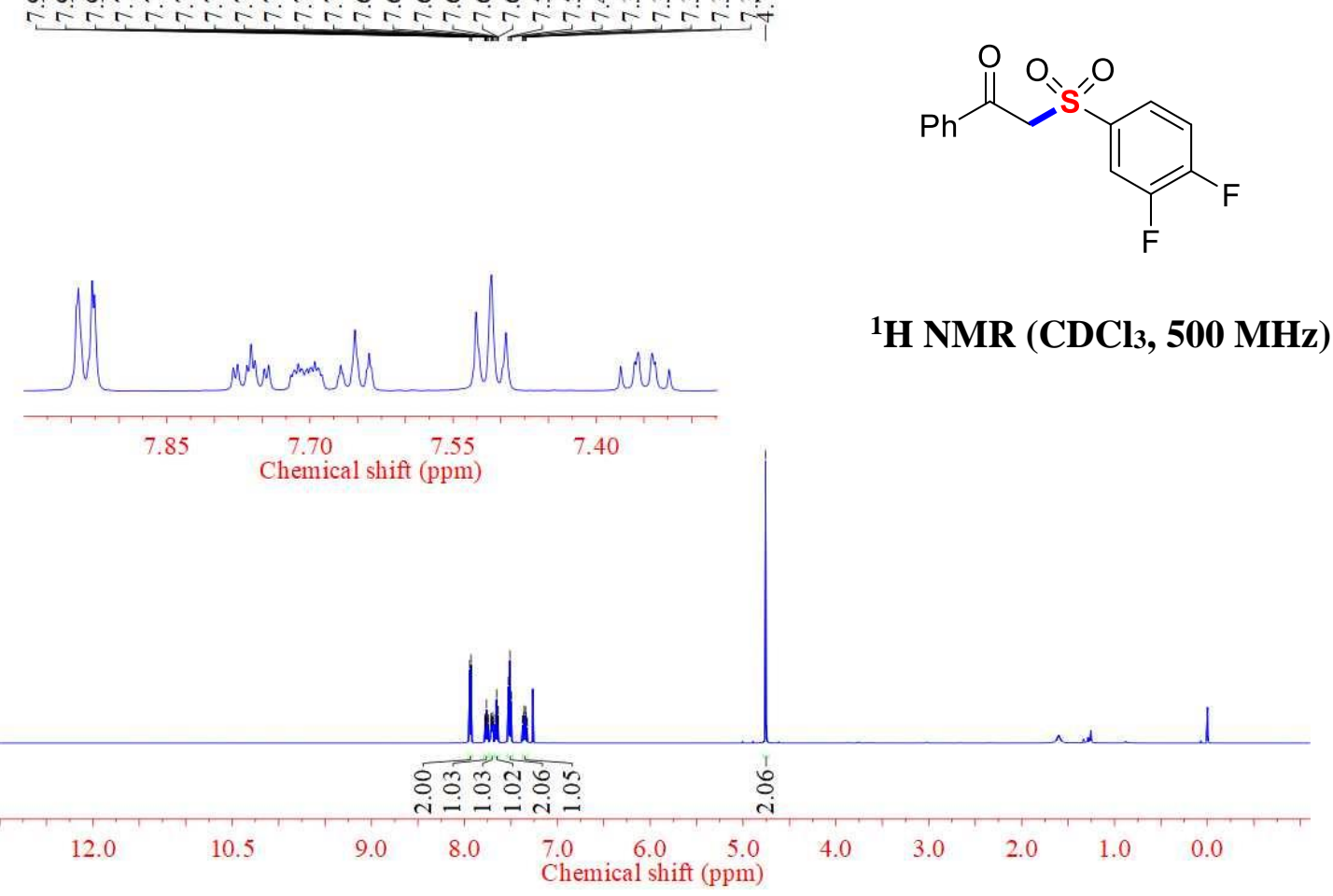

${ }^{1}$ H NMR Spectrum of Compound 3ap

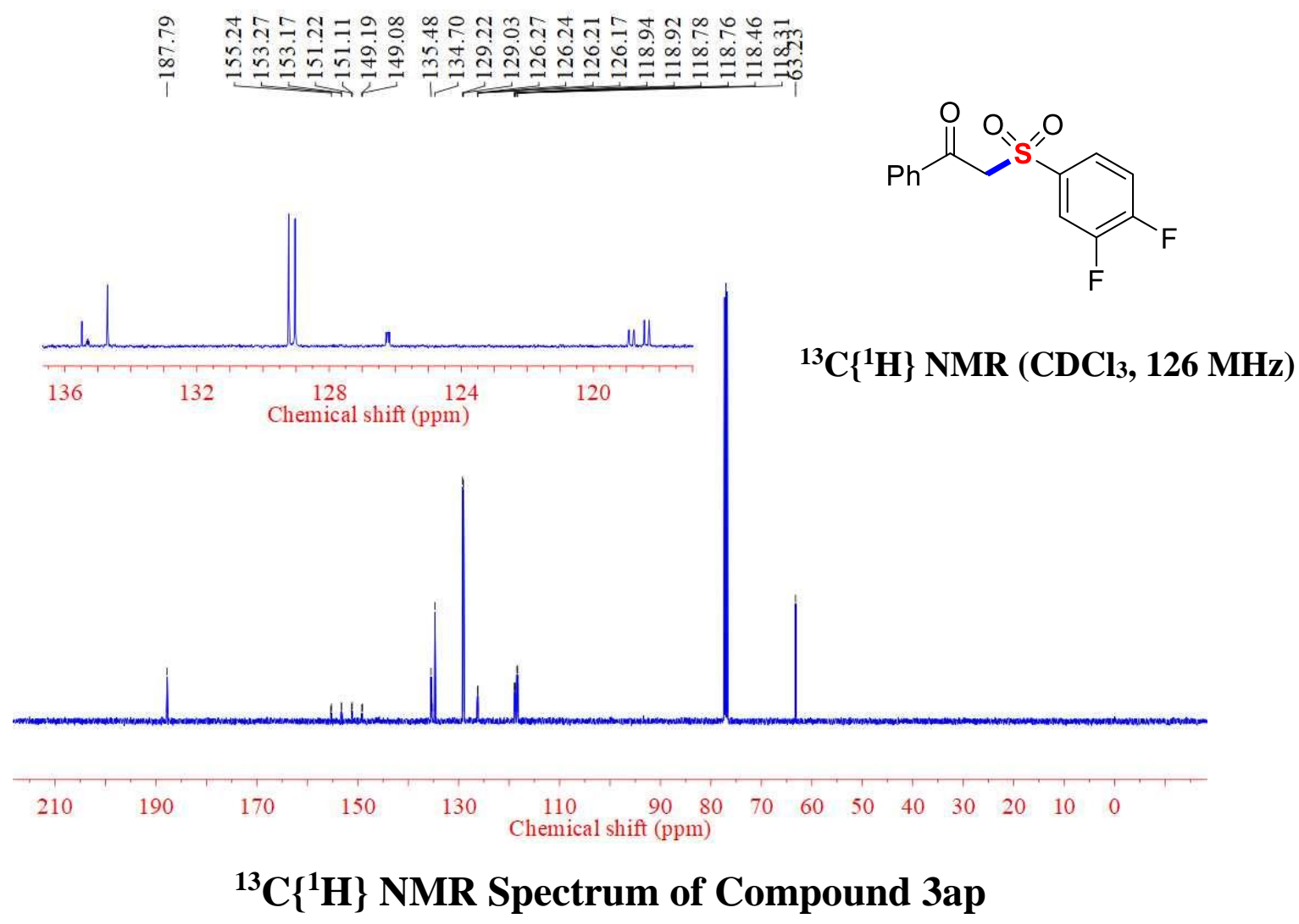


<smiles>O=C(CS(=O)(=O)c1ccc(F)c(F)c1)c1ccccc1</smiles>

${ }^{19} \mathrm{~F}\left\{{ }^{1} \mathrm{H}\right\}$ NMR $\left(\mathrm{CDCl}_{3}, 471 \mathrm{MHz}\right)$

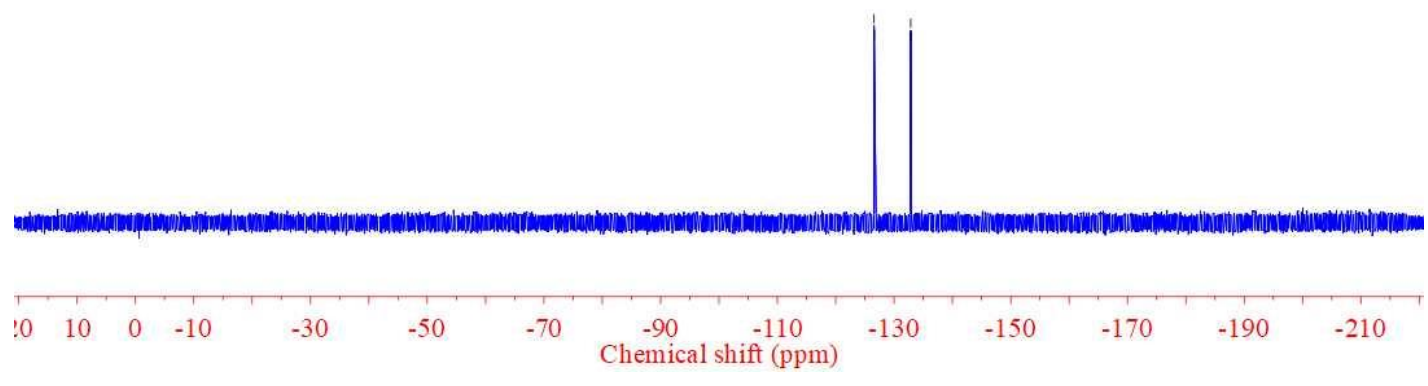

${ }^{19} \mathrm{~F}\left\{{ }^{1} \mathrm{H}\right\}$ NMR Spectrum of Compound 3ap

\section{Mass Spectrum SmartFormula Report}

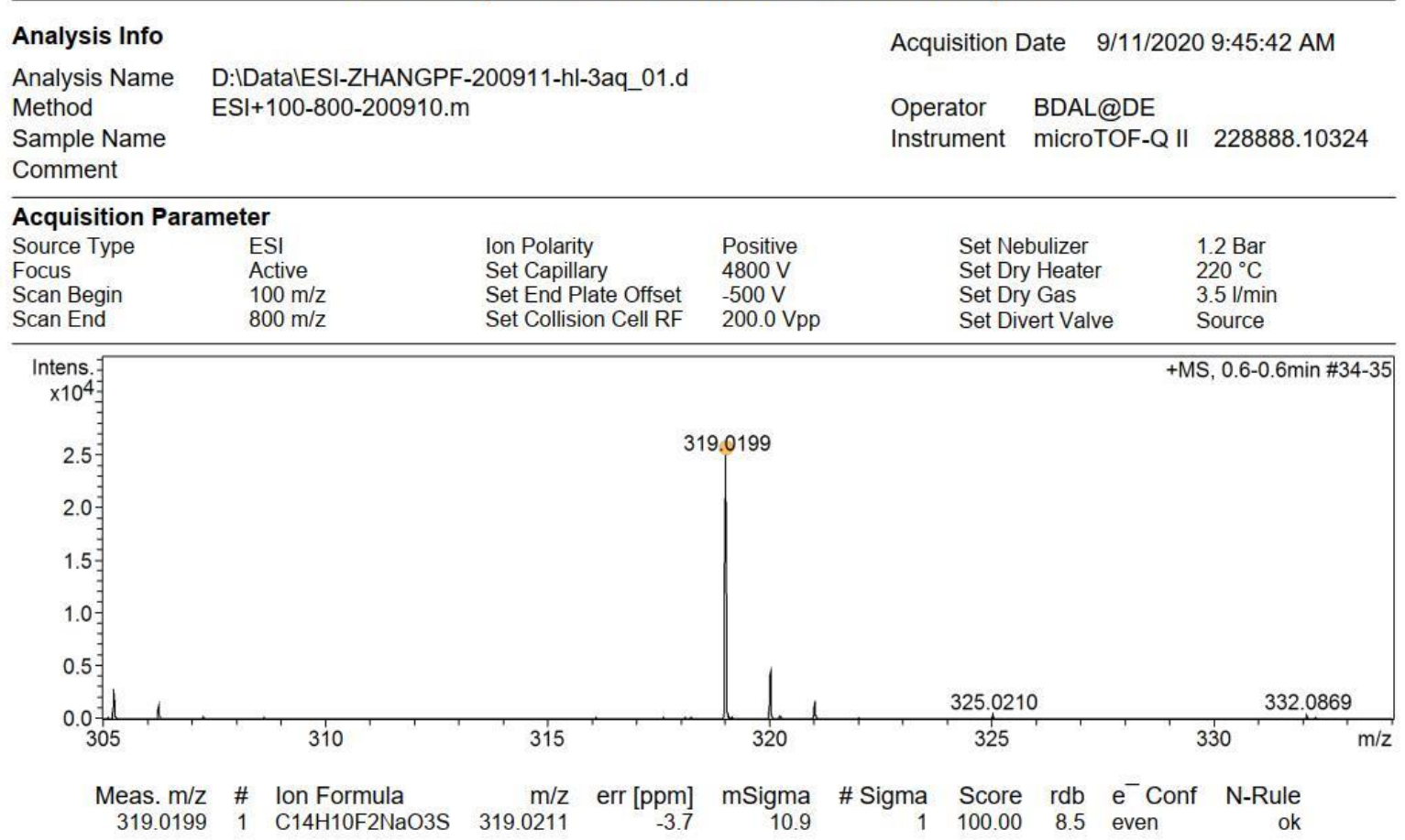

\section{HRMS Spectrum of Compound 3ap}


<smiles>O=C(CS(=O)(=O)c1ccc(Br)cc1F)c1ccccc1</smiles>

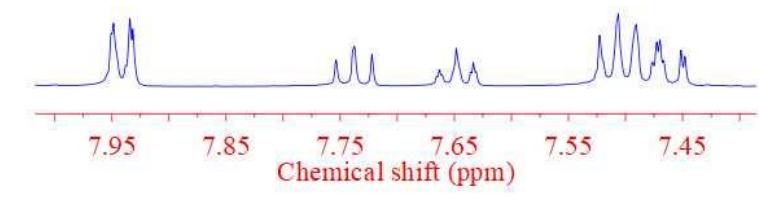

${ }^{1} \mathrm{H}$ NMR (CDCl3, $\left.500 \mathrm{MHz}\right)$

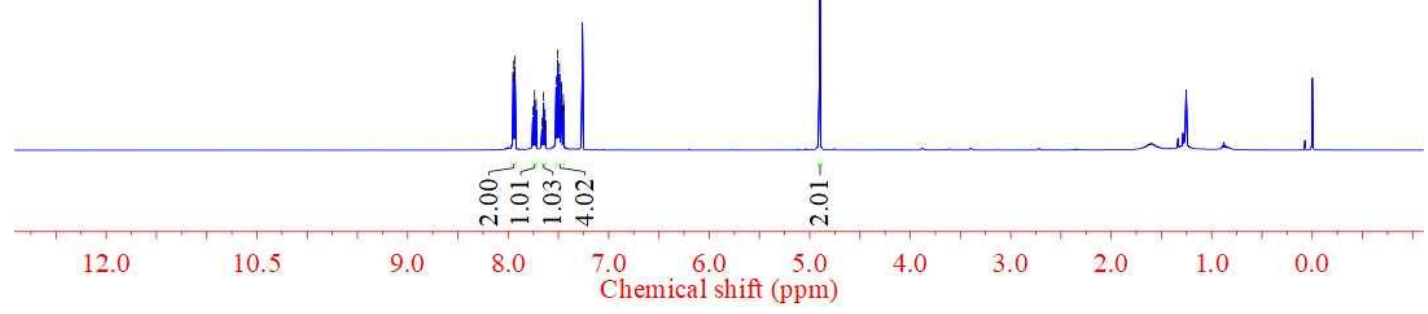

${ }^{1}$ H NMR Spectrum of Compound 3aq

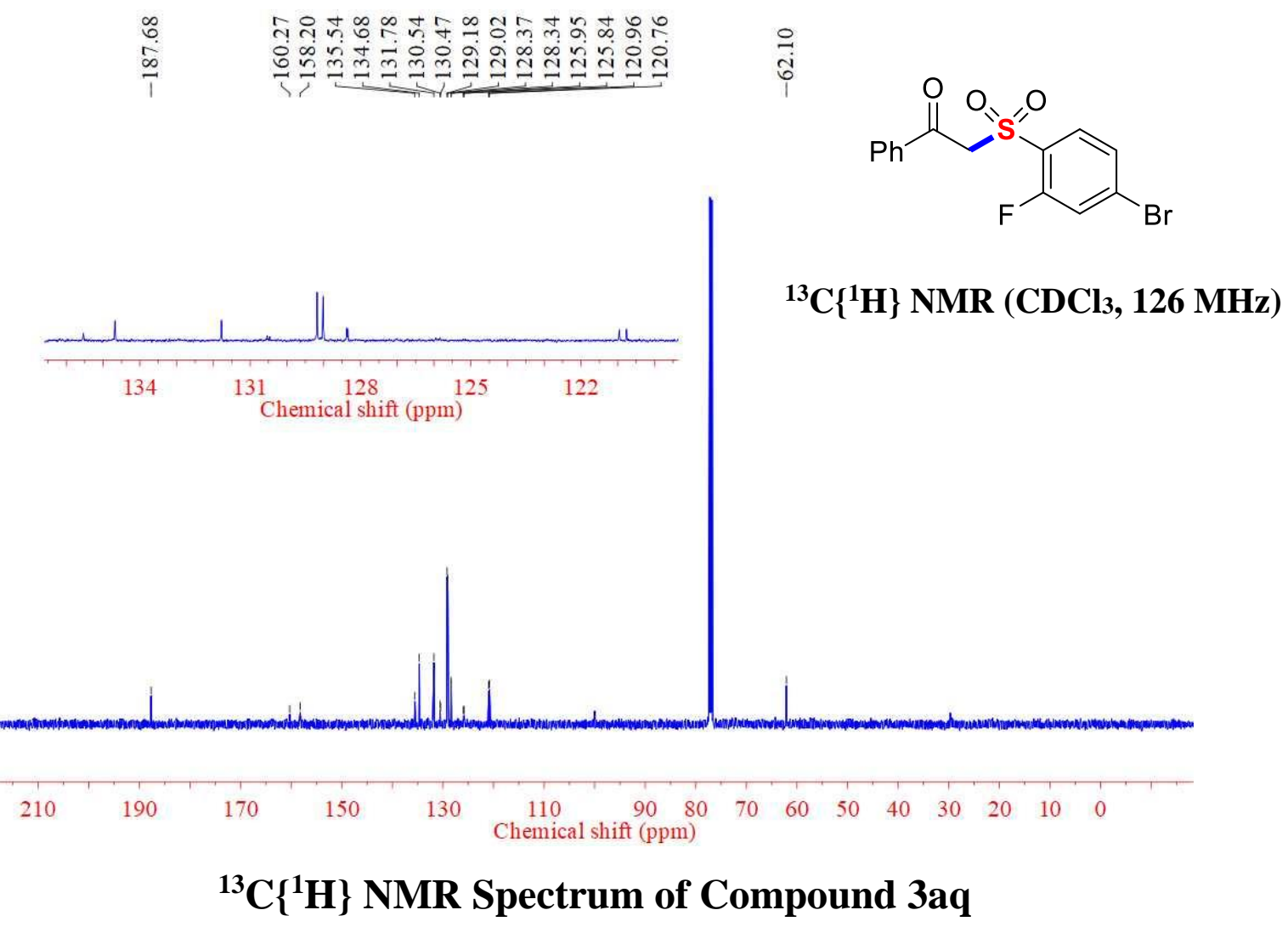


0
$\vdots$
$\vdots$
$\vdots$
$i$<smiles>O=C(CS(=O)(=O)c1ccc(Br)cc1F)c1ccccc1</smiles>

${ }^{19} \mathrm{~F}\left\{{ }^{1} \mathrm{H}\right\}$ NMR $\left(\mathrm{CDCl}_{3}, 471 \mathrm{MHz}\right)$

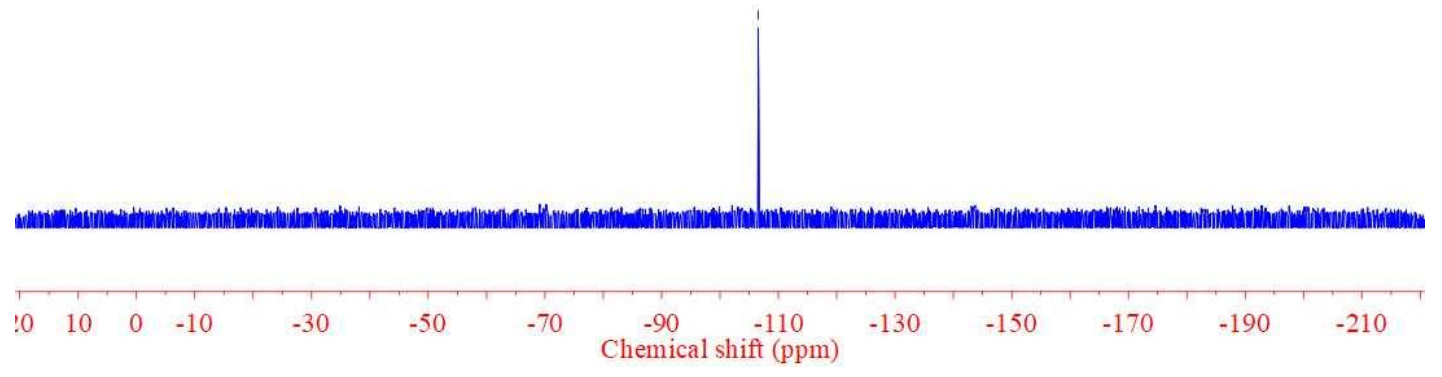

${ }^{19} \mathrm{~F}\left\{{ }^{1} \mathrm{H}\right\}$ NMR Spectrum of Compound 3aq

\section{Mass Spectrum SmartFormula Report}

\section{Analysis Info}

Analysis Name

Method

Sample Name

Comment
Acquisition Date $\quad 9 / 11 / 2020$ 9:47:35 AM

Operator BDAL@DE

Instrument / Ser\# microTOF-Q II 228888.10

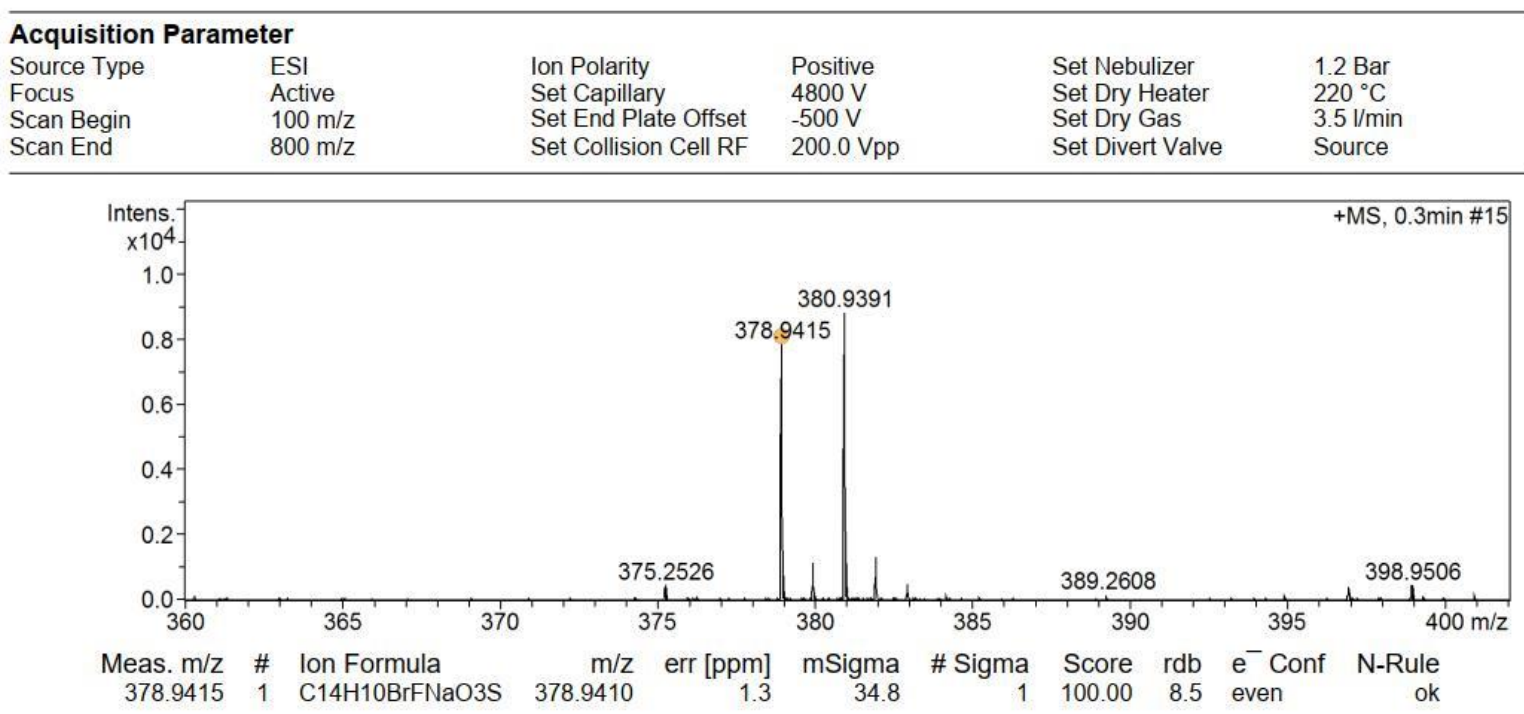

HRMS Spectrum of Compound 3aq 
<smiles>O=C(CS(=O)(=O)C1CC1)c1ccccc1</smiles>

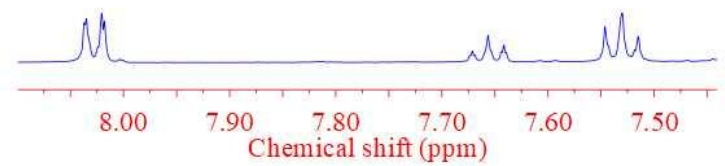

${ }^{1} \mathrm{H} \mathrm{NMR}\left(\mathrm{CDCl}_{3}, 500 \mathrm{MHz}\right)$

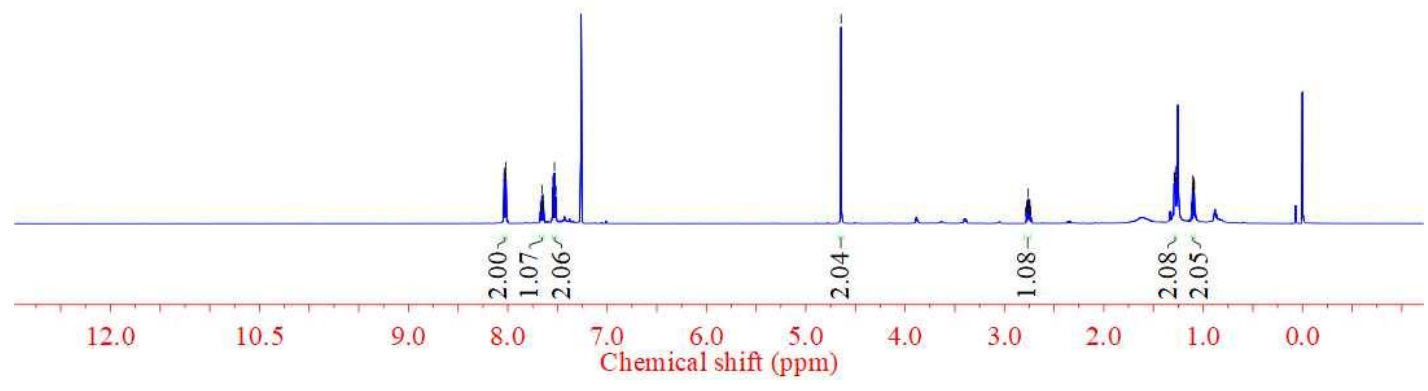

${ }^{1} \mathrm{H}$ NMR Spectrum of Compound 3ar

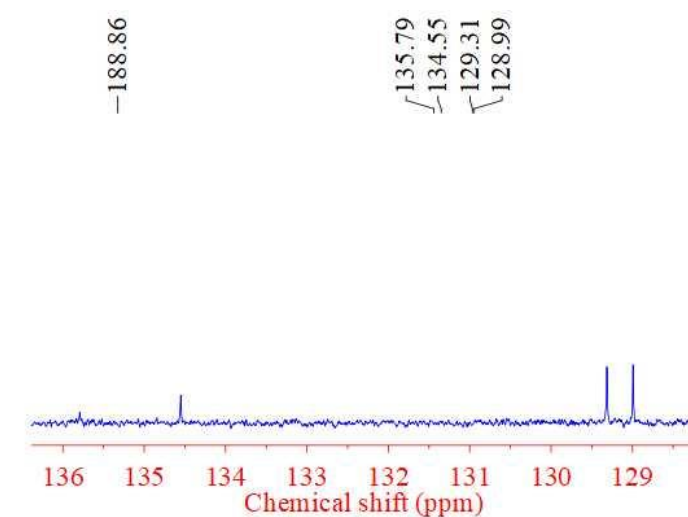

$\begin{array}{lll}n & n & + \\ 0 & 0 & n \\ i & 0 & n\end{array}$

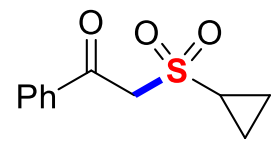

${ }^{13} \mathrm{C}\left\{{ }^{1} \mathrm{H}\right\}$ NMR $\left(\mathrm{CDCl}_{3}, 126 \mathrm{MHz}\right)$

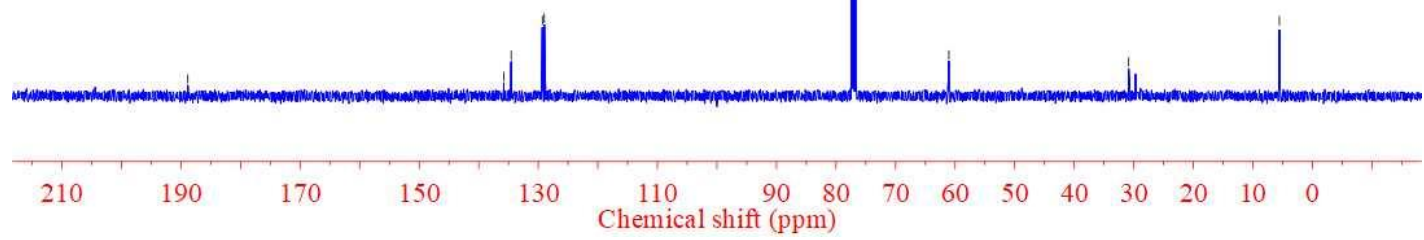

${ }^{13} \mathrm{C}\left\{{ }^{1} \mathrm{H}\right\}$ NMR Spectrum of Compound 3ar 
<smiles>CCS(=O)(=O)CC(=O)c1ccccc1</smiles>

${ }^{1} \mathrm{H}$ NMR $\left(\mathrm{CDCl}_{3}, 500 \mathrm{MHz}\right)$
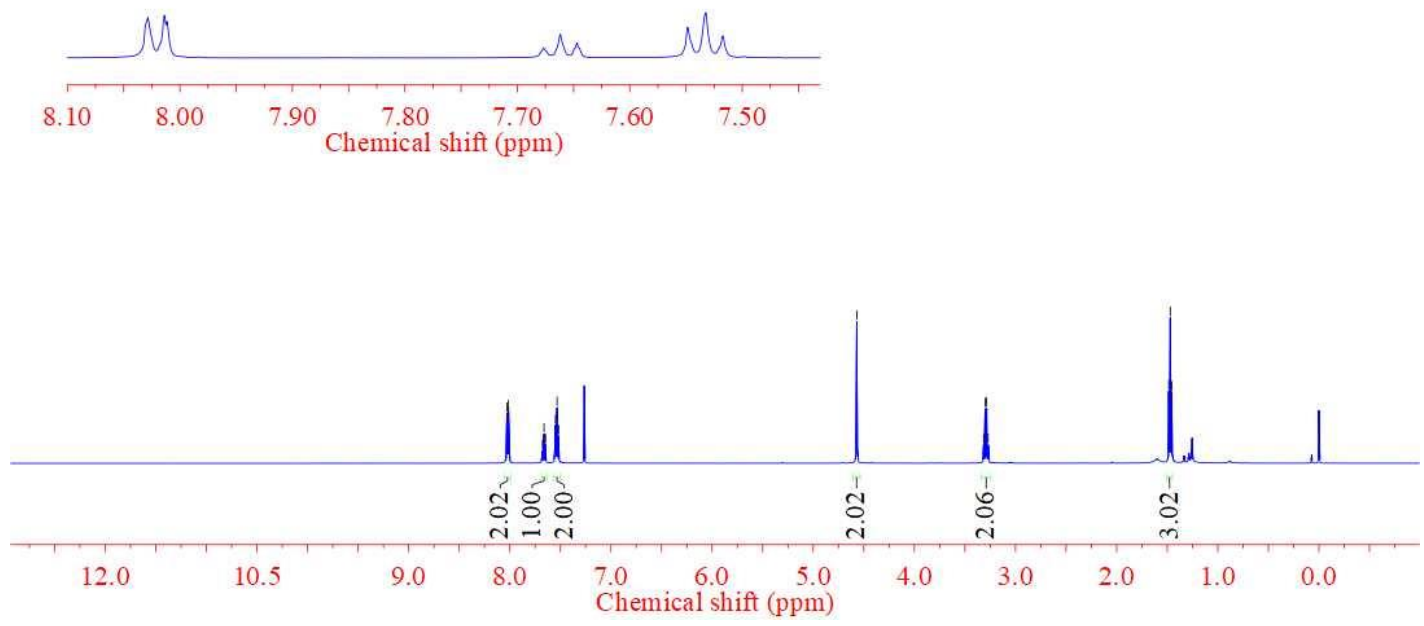

${ }^{1}$ H NMR Spectrum of Compound 3as

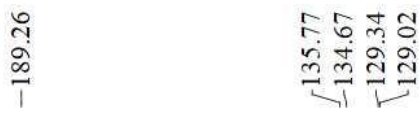

$\begin{array}{lll}1 & 0 \\ \infty & 1 & + \\ \infty & \infty & 0\end{array}$<smiles>CCS(=O)(=O)CC(=O)c1ccccc1</smiles>

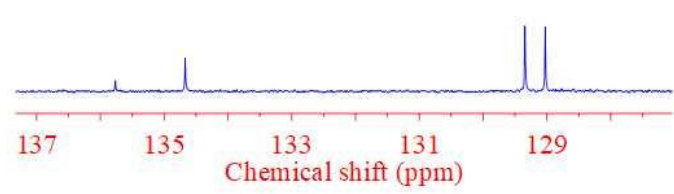

${ }^{13} \mathrm{C}\left\{{ }^{1} \mathrm{H}\right\} \mathrm{NMR}\left(\mathrm{CDCl}_{3}, 126 \mathrm{MHz}\right)$

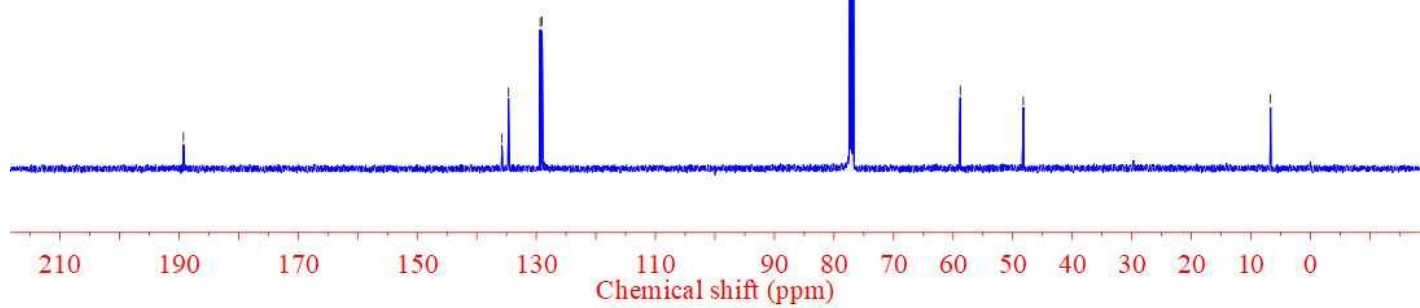

${ }^{13} \mathrm{C}\left\{{ }^{1} \mathrm{H}\right\}$ NMR Spectrum of Compound 3as 
<smiles>CCCS(=O)(O)(O)CC(=O)c1ccccc1</smiles>

${ }^{1} \mathrm{H}$ NMR (CDCl3, $\left.500 \mathrm{MHz}\right)$

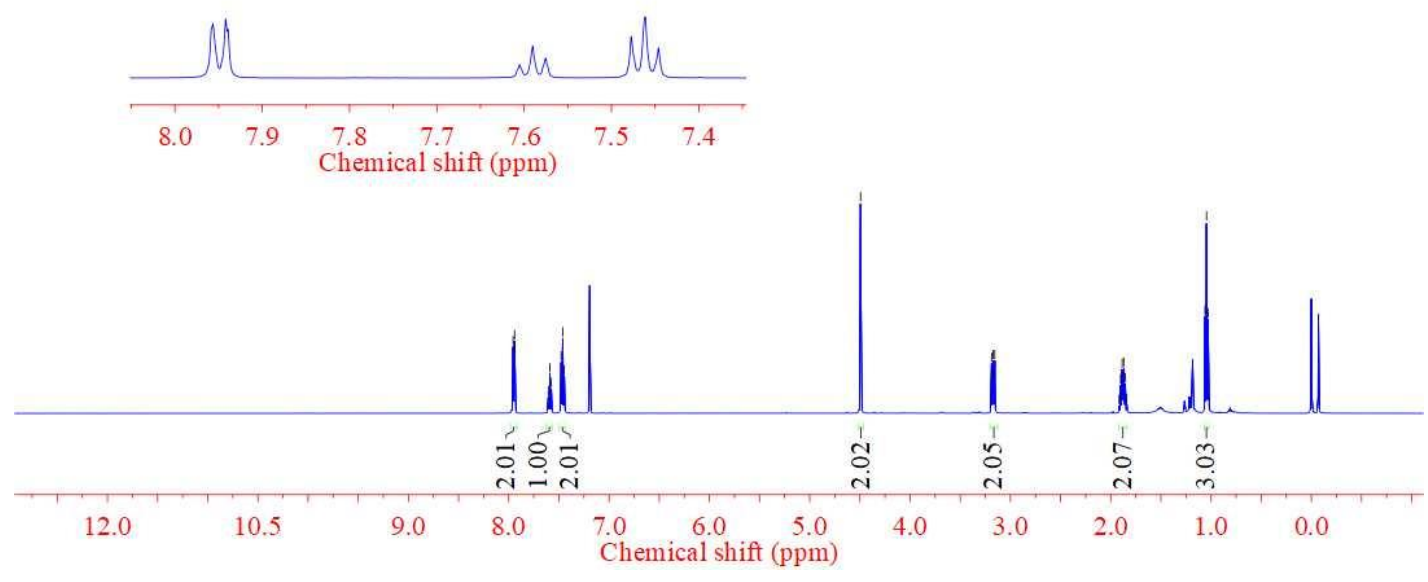

${ }^{1} \mathrm{H}$ NMR Spectrum of Compound 3at

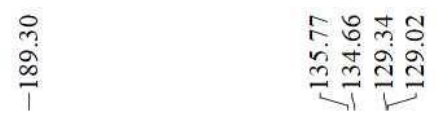

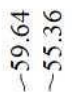

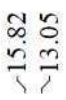<smiles>CCCS(=O)(=O)CC(=O)c1ccccc1</smiles>

${ }^{13} \mathrm{C}\left\{{ }^{1} \mathrm{H}\right\}$ NMR $\left(\mathrm{CDCl}_{3}, 126 \mathrm{MHz}\right)$
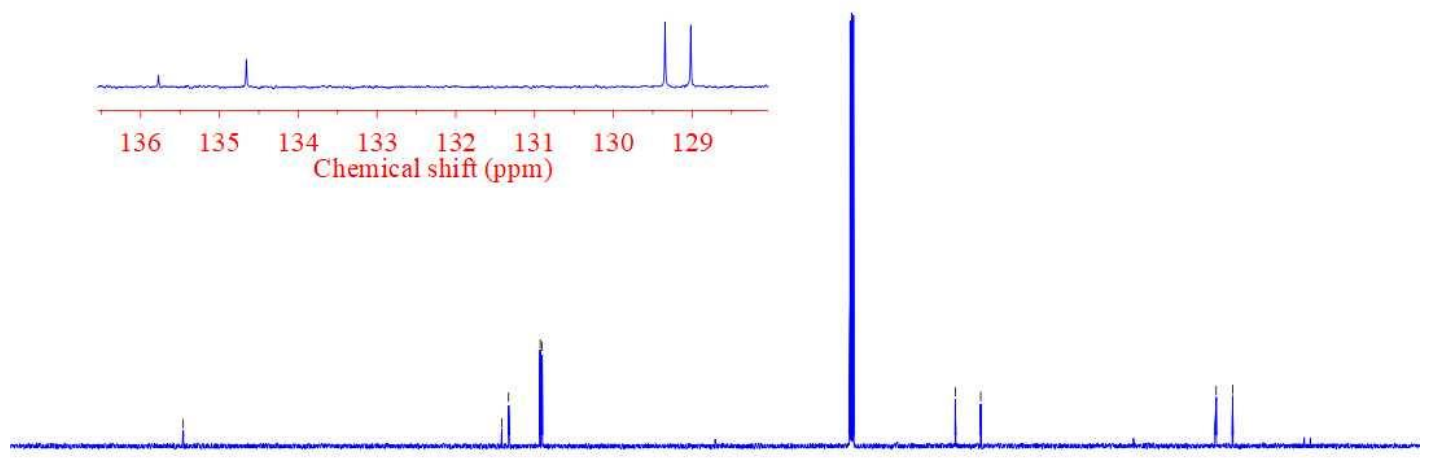

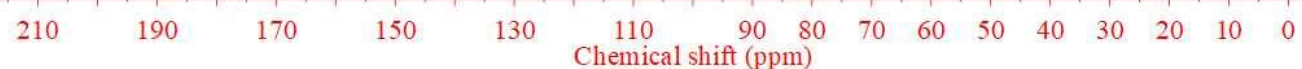

${ }^{13} \mathrm{C}\left\{{ }^{1} \mathrm{H}\right\}$ NMR Spectrum of Compound 3at 


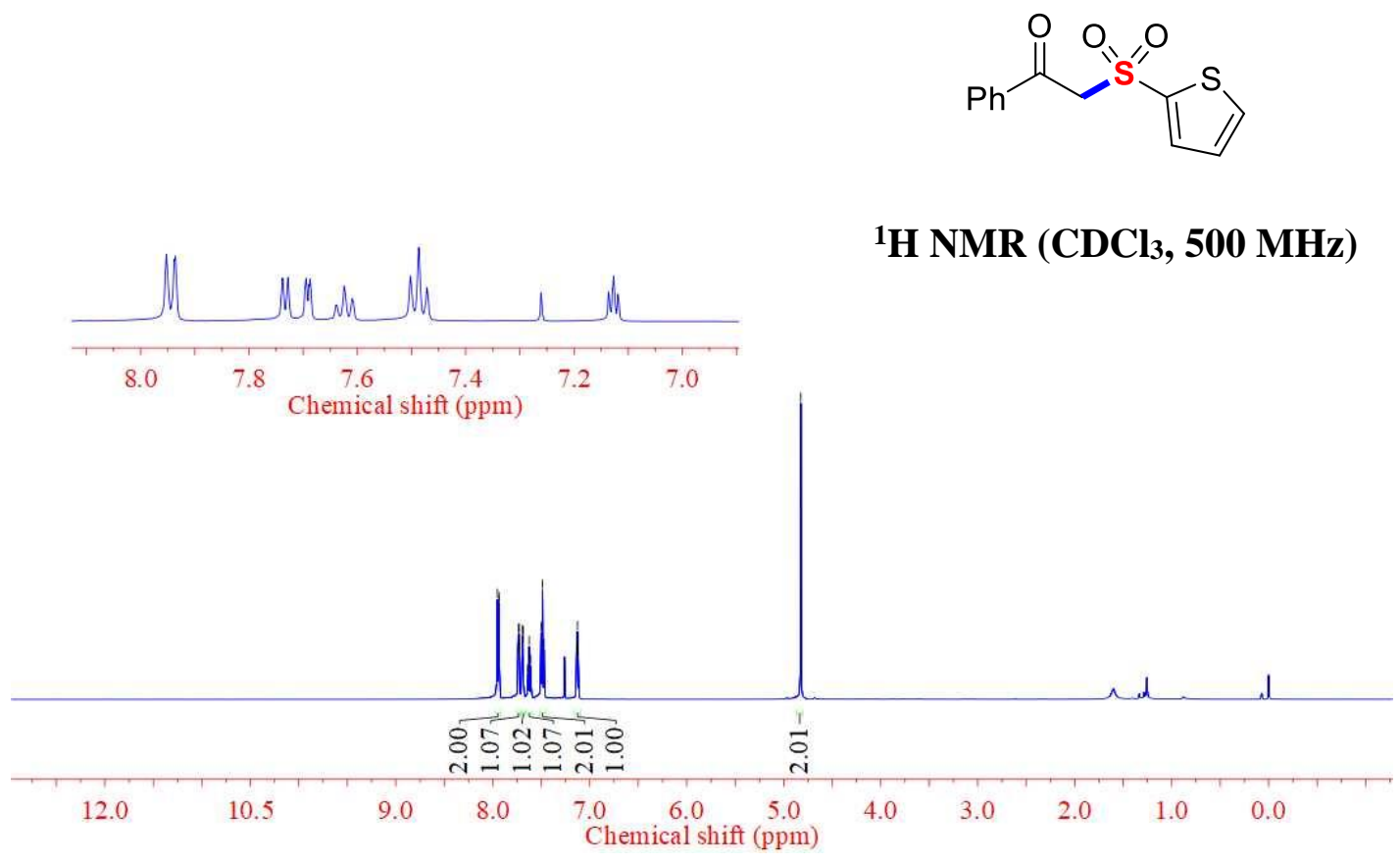

${ }^{1}$ H NMR Spectrum of Compound 3au

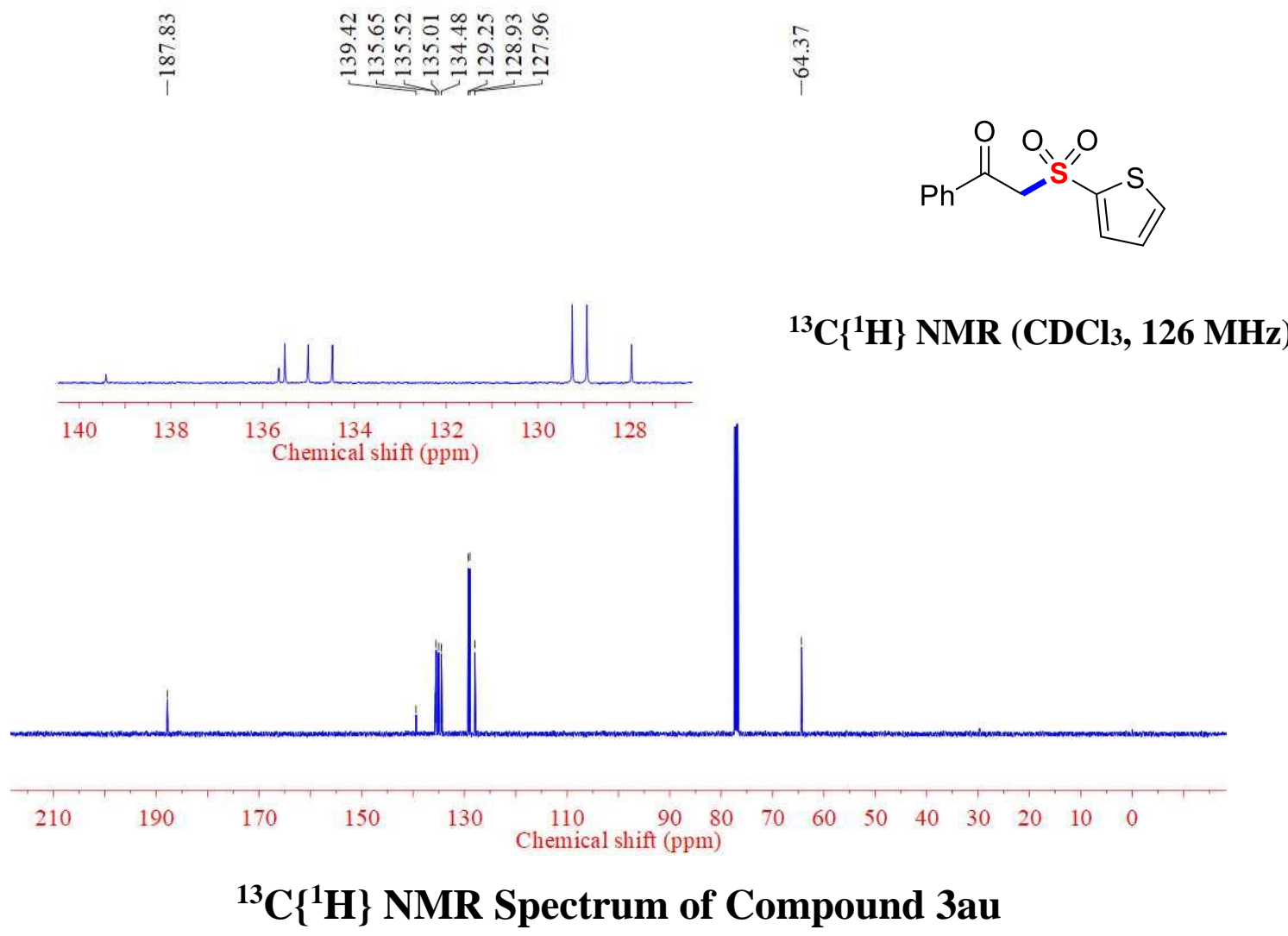


<smiles>O=C(CS(=O)(=O)c1ccc(Cl)s1)c1ccccc1</smiles>

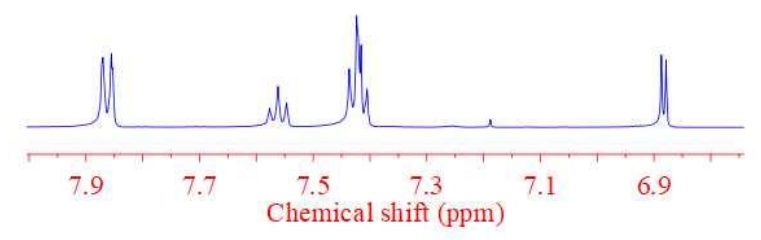

${ }^{1} \mathrm{H}$ NMR $\left(\mathrm{CDCl}_{3}, 500 \mathrm{MHz}\right)$

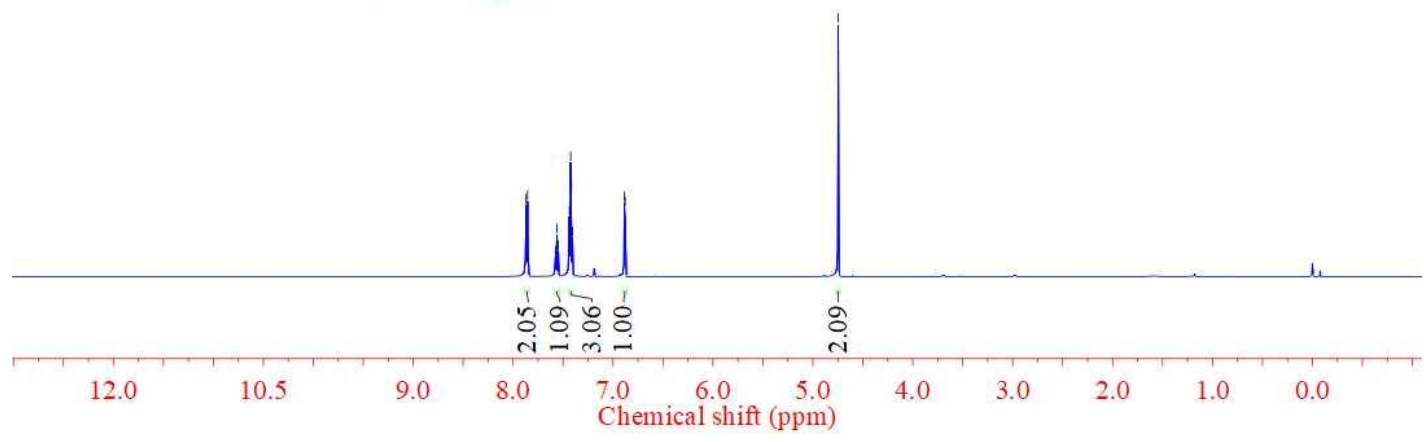

${ }^{1}$ H NMR Spectrum of Compound 3av

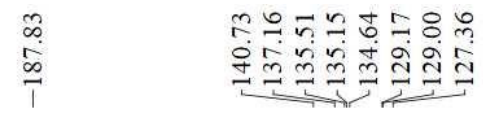

it<smiles>O=C(CS(=O)(=O)c1ccc(Cl)s1)c1ccccc1</smiles>

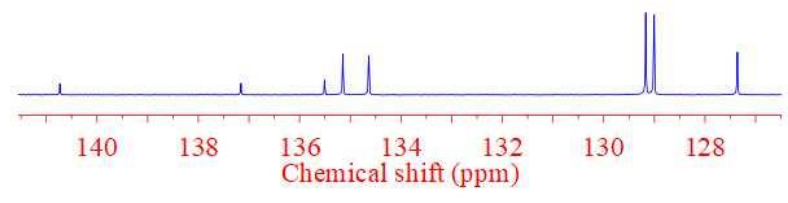

${ }^{13} \mathrm{C}\left\{{ }^{1} \mathrm{H}\right\}$ NMR $\left(\mathrm{CDCl}_{3}, 126 \mathrm{MHz}\right)$

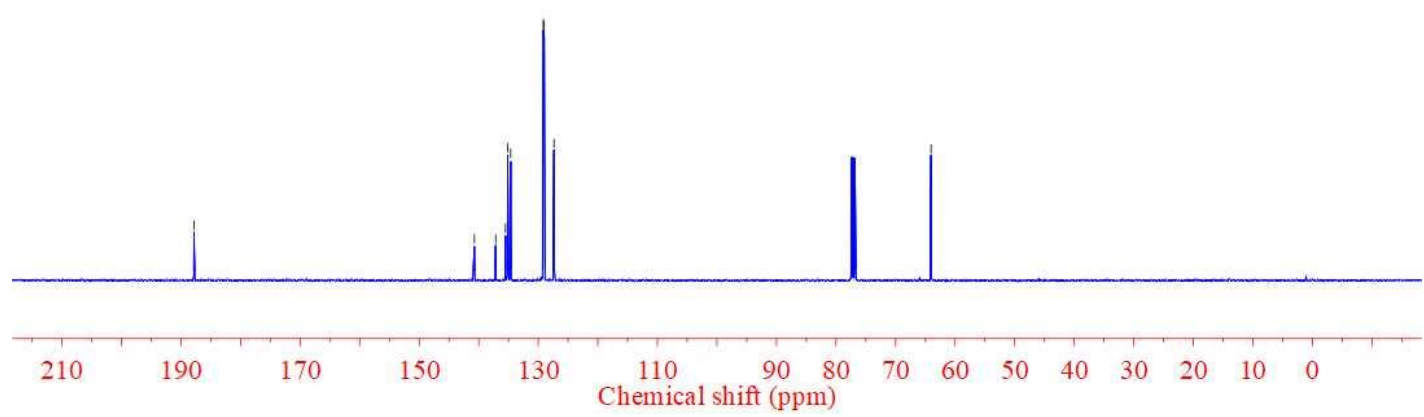

${ }^{13} \mathrm{C}\left\{{ }^{1} \mathrm{H}\right\}$ NMR Spectrum of Compound 3av 


\section{Mass Spectrum SmartFormula Report}

Analysis Info

Analysis Name

Method

Sample Name

Comment
Acquisition Date $\quad$ 9/11/2020 9:49:52 AM

D:IDatalESI-ZHANGPF-200911-hl-3aw_01.d

ESI+100-800-200910 m

Operator BDAL@DE

Instrument microTOF-Q II 228888.10324

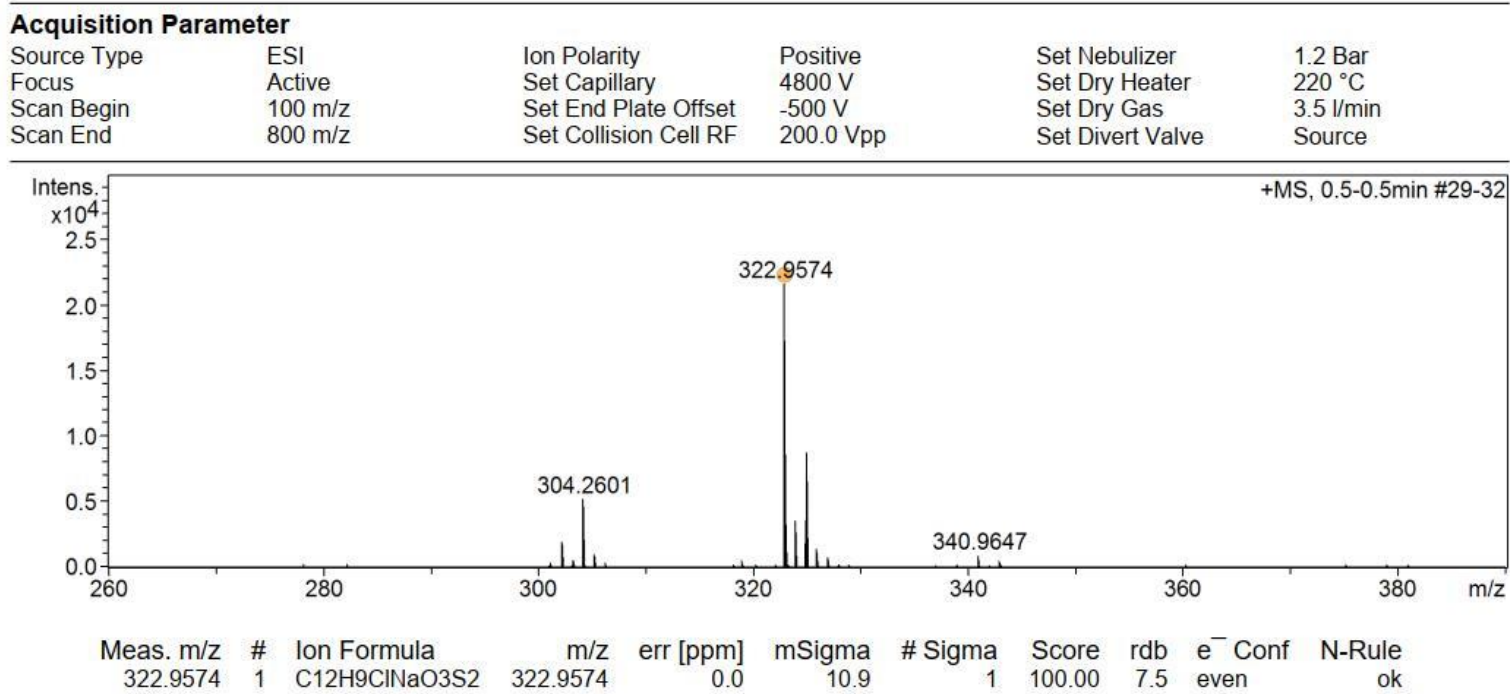

HRMS Spectrum of Compound 3av

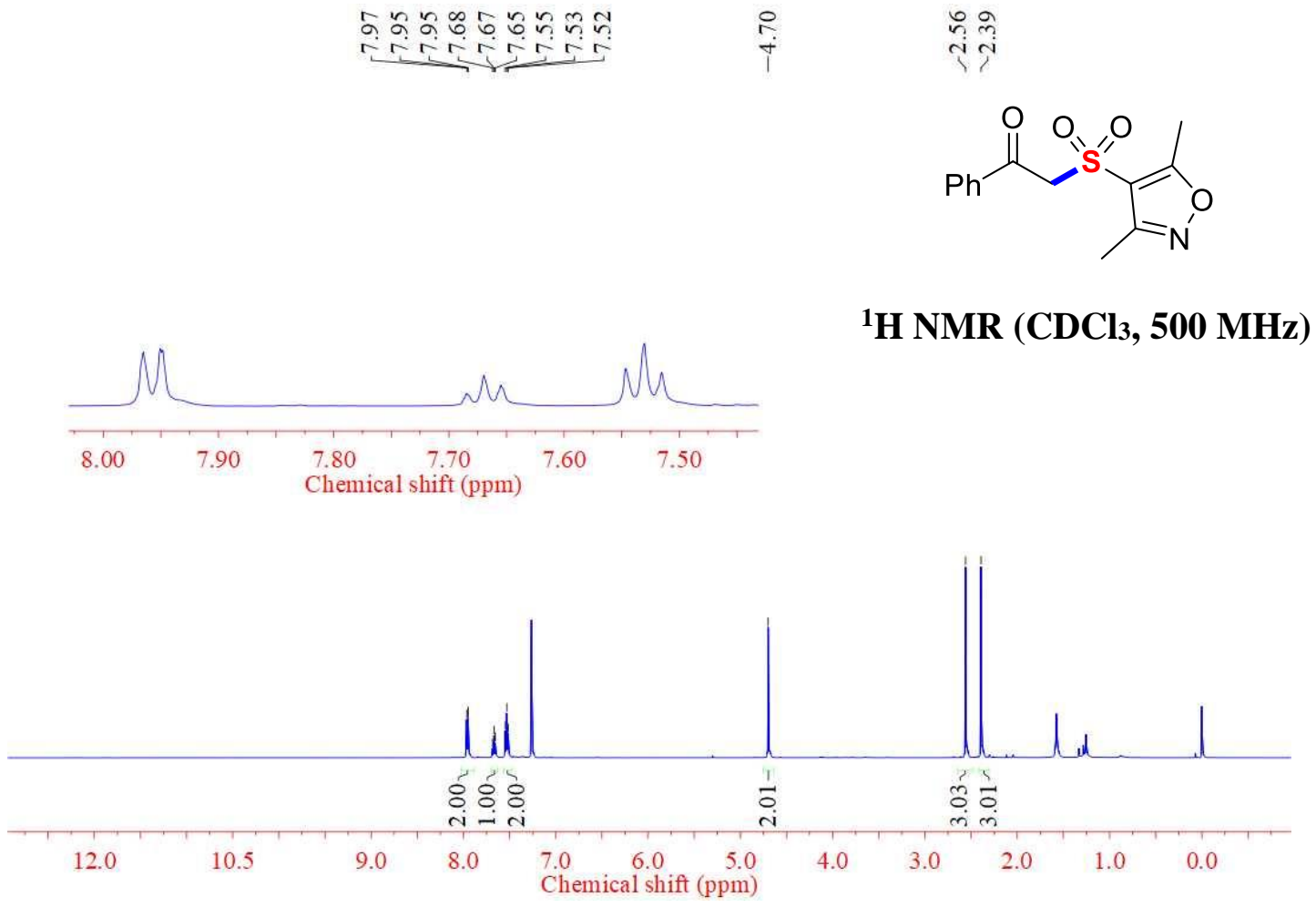

${ }^{1}$ H NMR Spectrum of Compound 3aw 


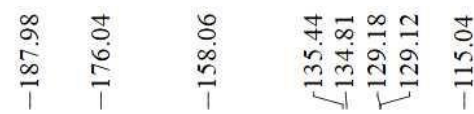
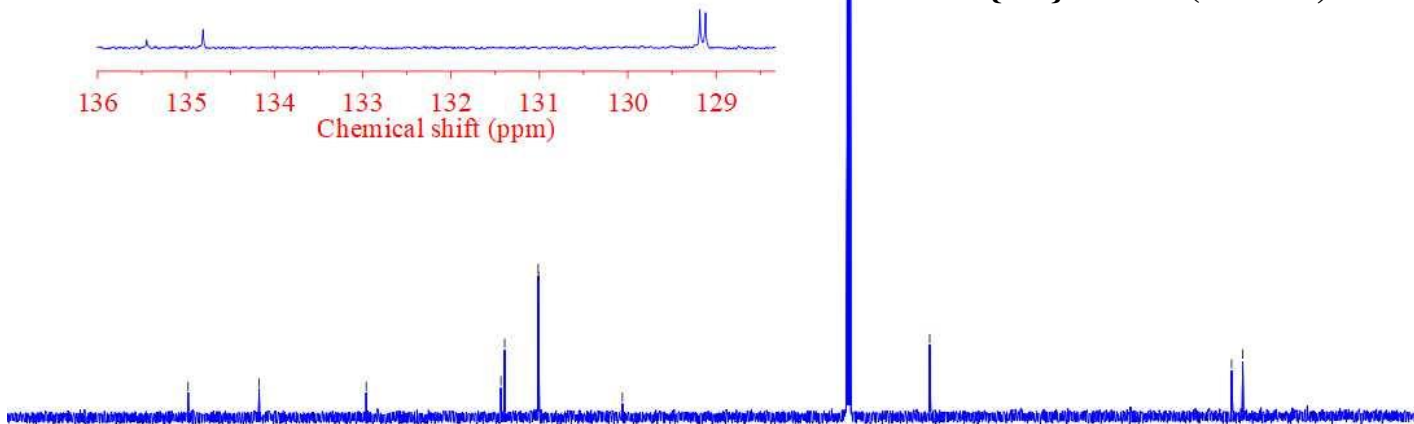

$\stackrel{m}{\tilde{n}}$

ㄷำ<smiles>Cc1noc(C)c1S(=O)(=O)CC(=O)c1ccccc1</smiles>

${ }^{13} \mathrm{C}\left\{{ }^{1} \mathrm{H}\right\}$ NMR $\left(\mathrm{CDCl}_{3}, 126 \mathrm{MHz}\right)$
210
190
150
130
110
9080
06
5040
3020
$\begin{array}{lll}0 & 10 & 0\end{array}$

\section{${ }^{13} \mathrm{C}\left\{{ }^{1} \mathrm{H}\right\}$ NMR Spectrum of Compound 3aw}

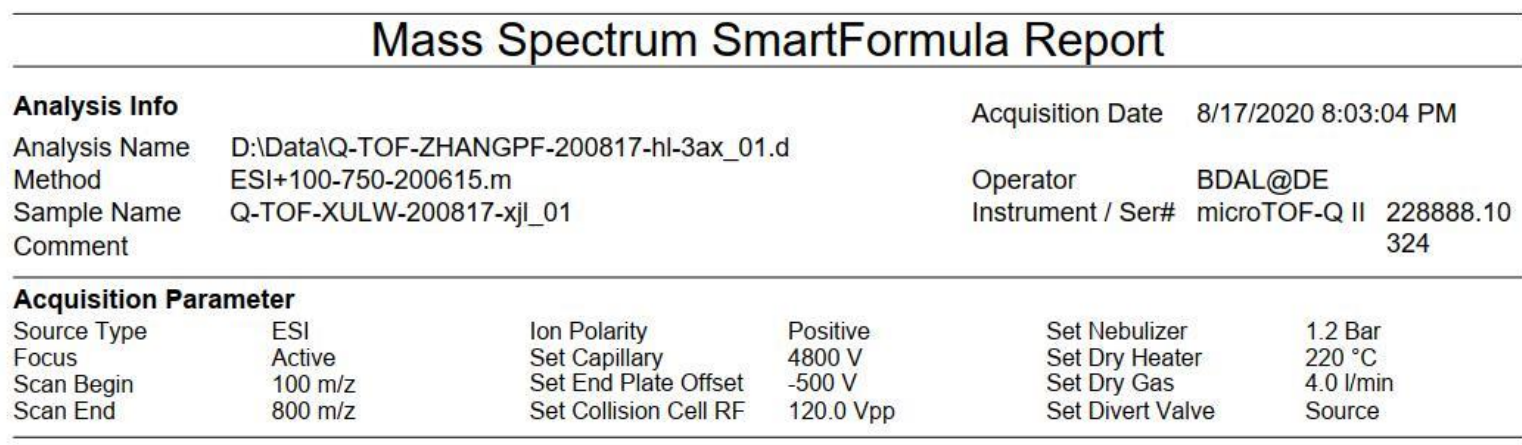

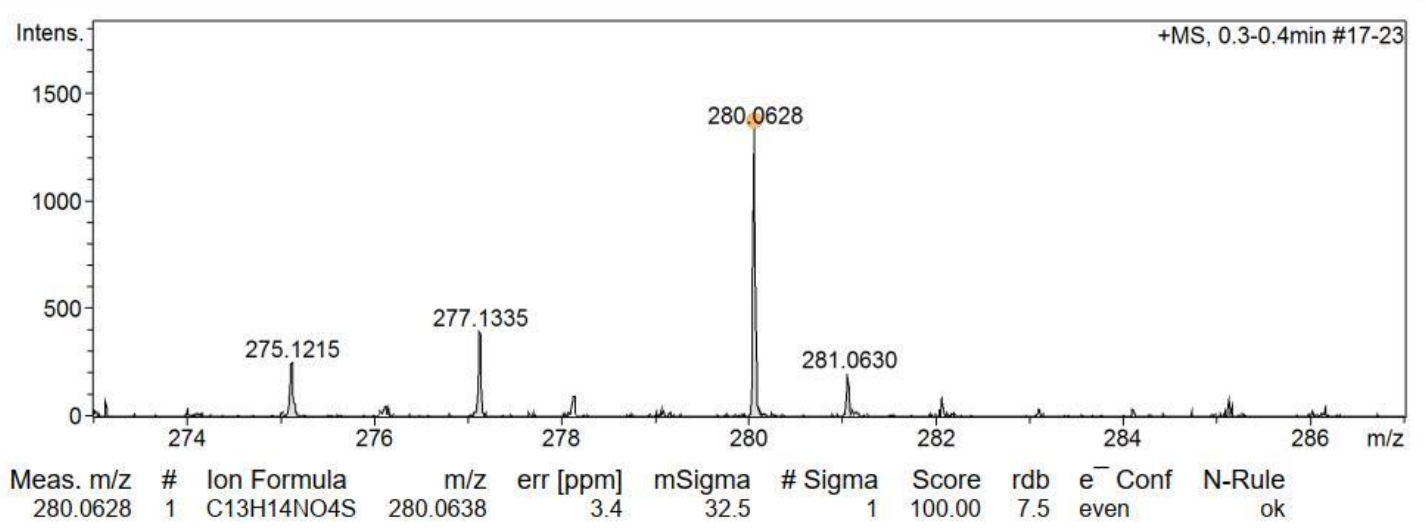

HRMS Spectrum of Compound 3aw 
<smiles>O=C(CS(=O)(=O)c1ccccc1)c1ccc(C(F)(F)F)cc1</smiles>

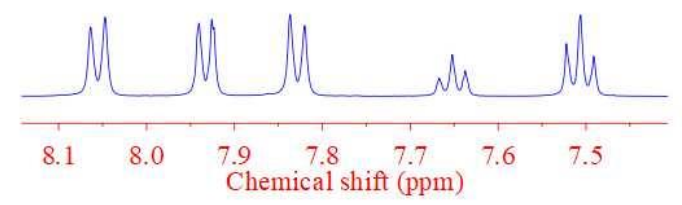

${ }^{1} \mathrm{H}$ NMR $\left(\mathrm{CDCl}_{3}, 500 \mathrm{MHz}\right)$

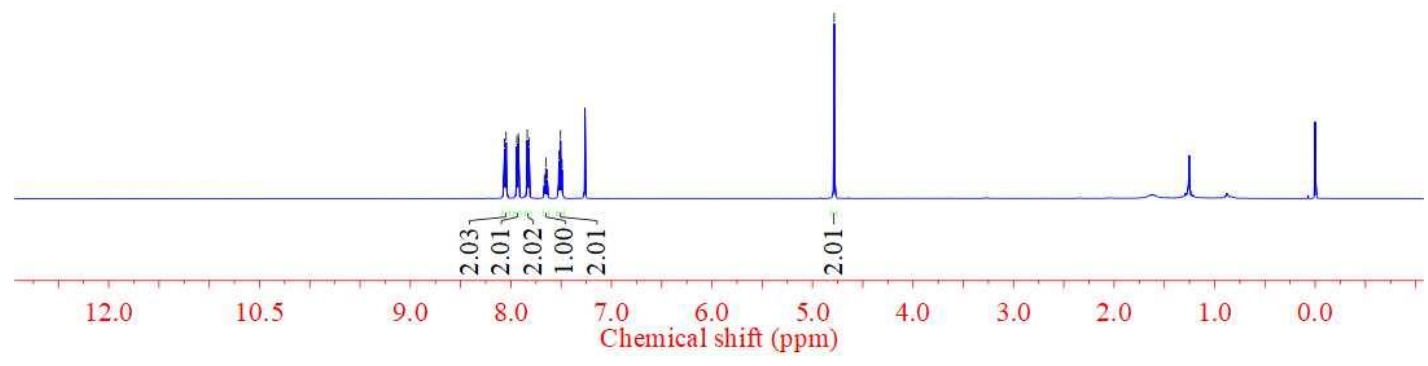

${ }^{1} \mathrm{H}$ NMR Spectrum of Compound 3ax

oे
के
1

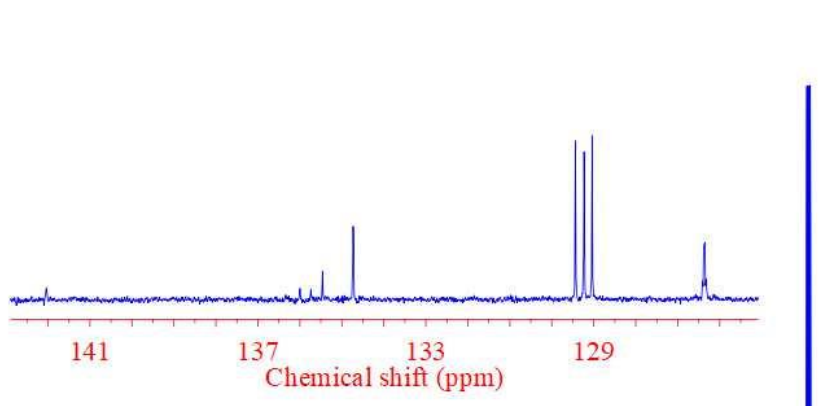<smiles>O=C(CS(=O)(=O)c1ccccc1)c1ccc(C(F)(F)F)cc1</smiles>

${ }^{13} \mathrm{C}\left\{{ }^{1} \mathrm{H}\right\}$ NMR $\left(\mathrm{CDCl}_{3}, 126 \mathrm{MHz}\right)$

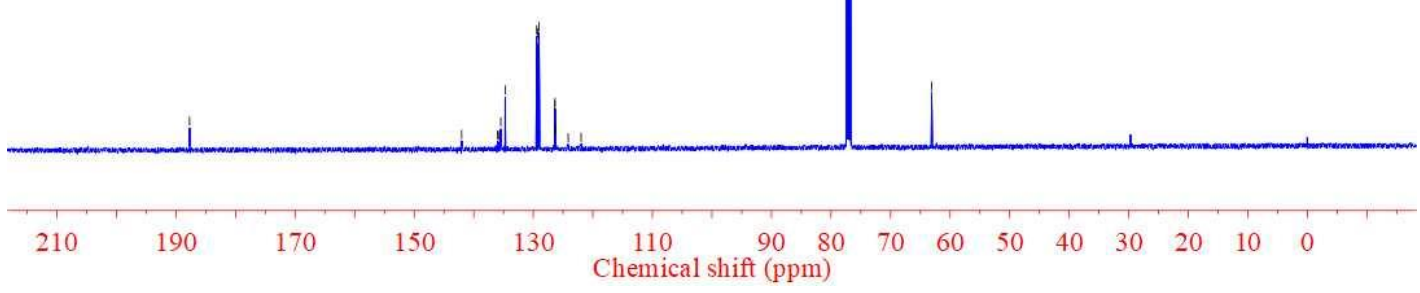

${ }^{13} \mathrm{C}\left\{{ }^{1} \mathrm{H}\right\}$ NMR Spectrum of Compound 3ax 
<smiles>O=C(CS(=O)(=O)c1ccccc1)c1ccc(C(F)(F)F)cc1</smiles>

${ }^{19} \mathrm{~F}\left\{{ }^{1} \mathrm{H}\right\}$ NMR $\left(\mathrm{CDCl}_{3}, 471 \mathrm{MHz}\right)$

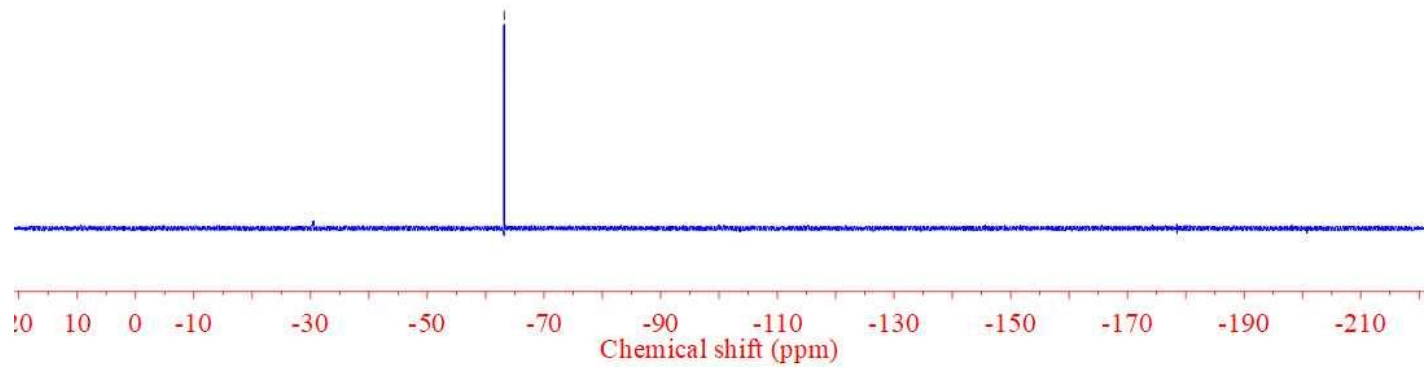

${ }^{19} \mathbf{F}\left\{{ }^{1} \mathbf{H}\right\}$ NMR Spectrum of Compound 3ax<smiles>O=C(c1ccccc1)N(N1CCOCC1)S(=O)(=O)c1ccccc1</smiles>

${ }^{1} \mathrm{H} \mathrm{NMR}\left(\mathrm{CDCl}_{3}, 500 \mathrm{MHz}\right)$

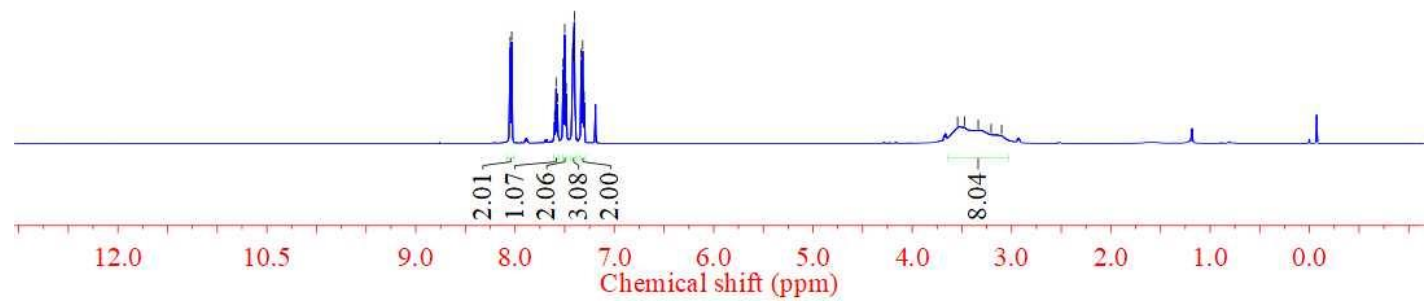

${ }^{1}$ H NMR Spectrum of Compound 5a 
<smiles>O=C(c1ccccc1)N(N1CCOCC1)S(=O)(=O)c1ccccc1</smiles>

${ }^{13} \mathrm{C}\left\{{ }^{1} \mathrm{H}\right\} \mathrm{NMR}\left(\mathrm{CDCl}_{3}, 126 \mathrm{MHz}\right)$

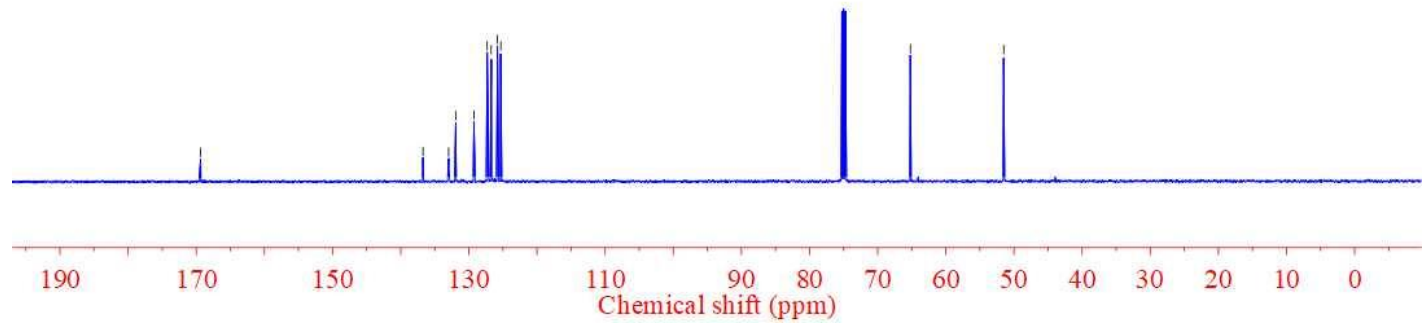

${ }^{13} \mathbf{C}\left\{{ }^{1} \mathbf{H}\right\}$ NMR Spectrum of Compound 5a

\section{Mass Spectrum SmartFormula Report}

Analysis Info

Method

Sample Name

D:IDatal2020|0804|3A_2-C,8_01_13762.d

Ic_pos_3min_hn.m

$3 \mathrm{~A}$

Comment
Analysis Name

Acquisition Parameter

Source Type

Focus

Scan Begin

Not active

$50 \mathrm{~m} / \mathrm{z}$

$950 \mathrm{~m} / \mathrm{z}$

Set Capillary

Set End Plate Offse

Set Collision Cell RF

Positive

$5500 \mathrm{~V}$

Acquisition Date

Operator

BDAL@DE

Instrument / Ser\# micrOTOF-Q II 10366

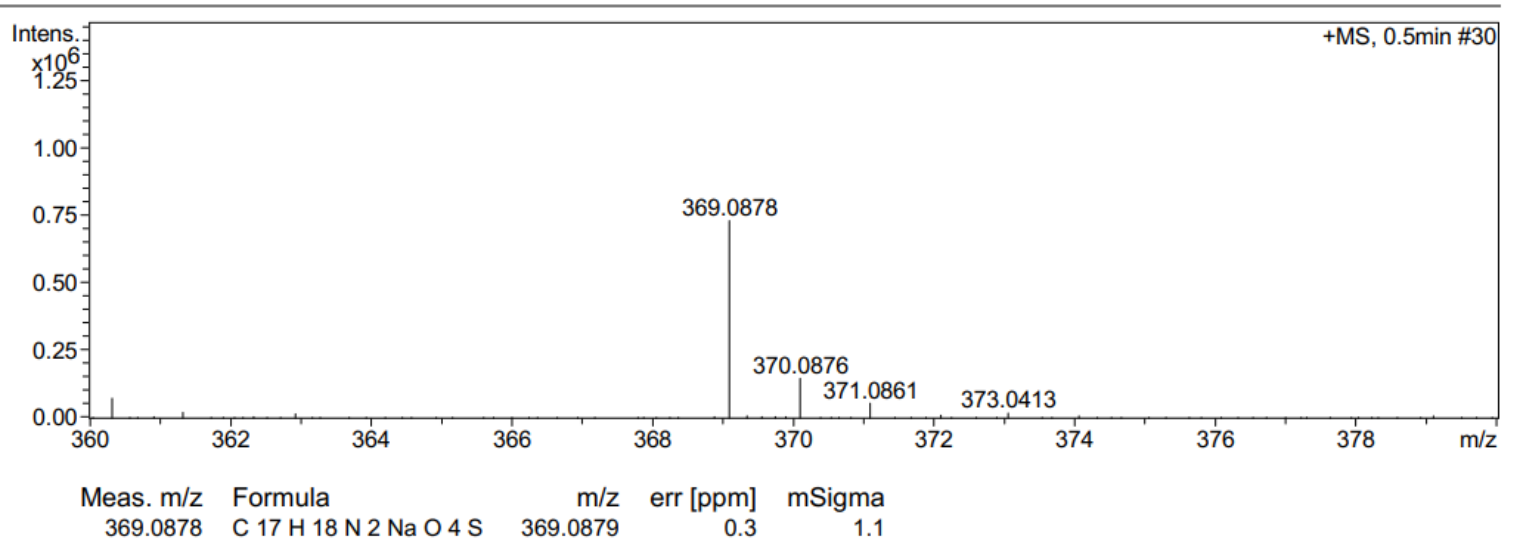

HRMS Spectrum of Compound 5a 


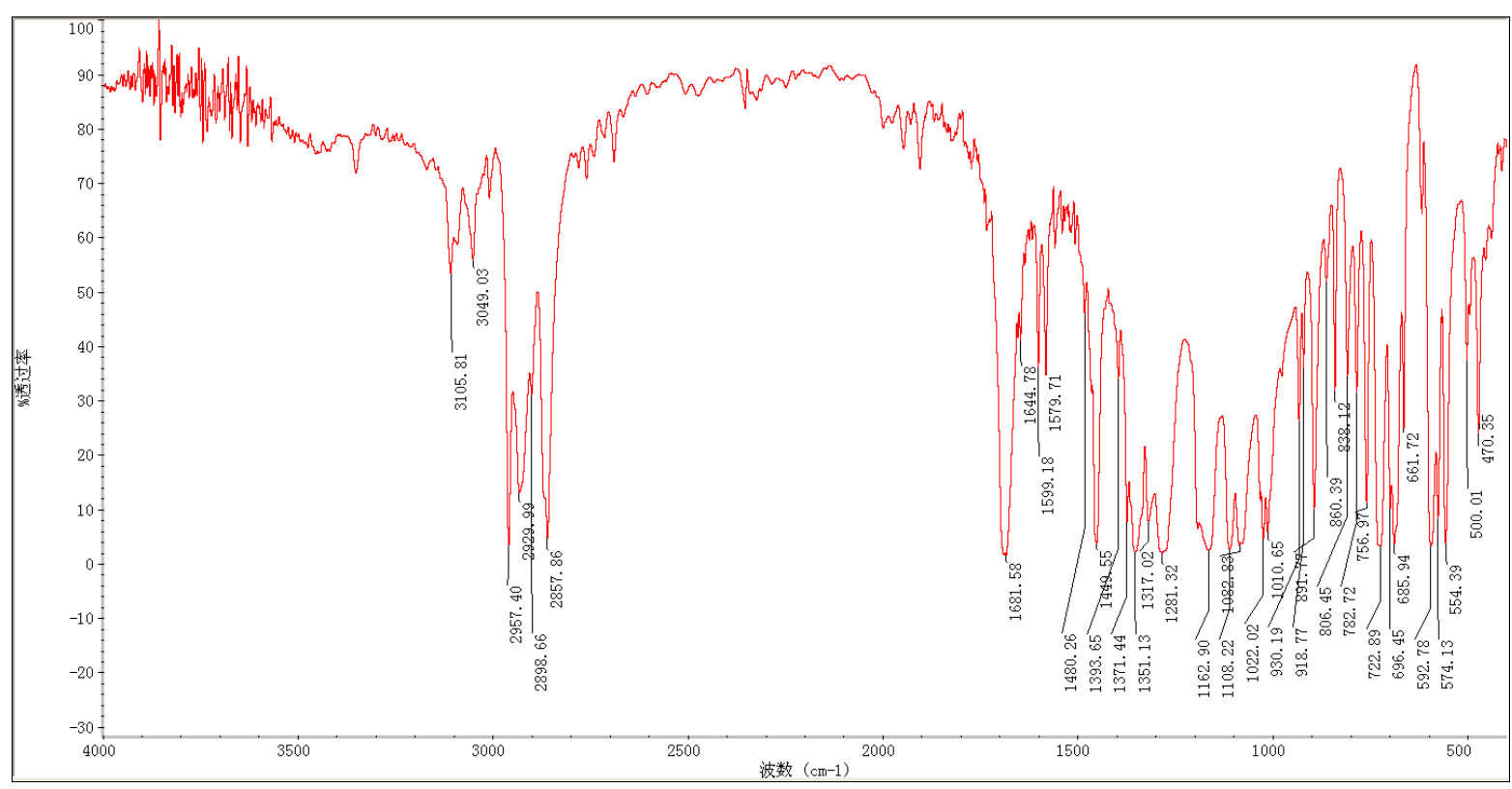

IR Spectrum of Compound 5a

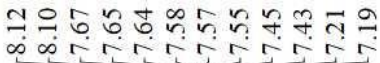

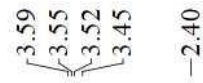<smiles>Cc1ccc(C(=O)N(N2CCOCC2)S(=O)(=O)c2ccccc2)cc1</smiles>

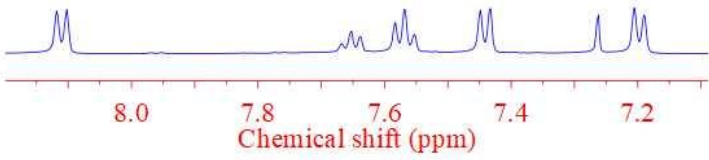

${ }^{1} \mathrm{H}$ NMR $\left(\mathrm{CDCl}_{3}, 500 \mathrm{MHz}\right)$

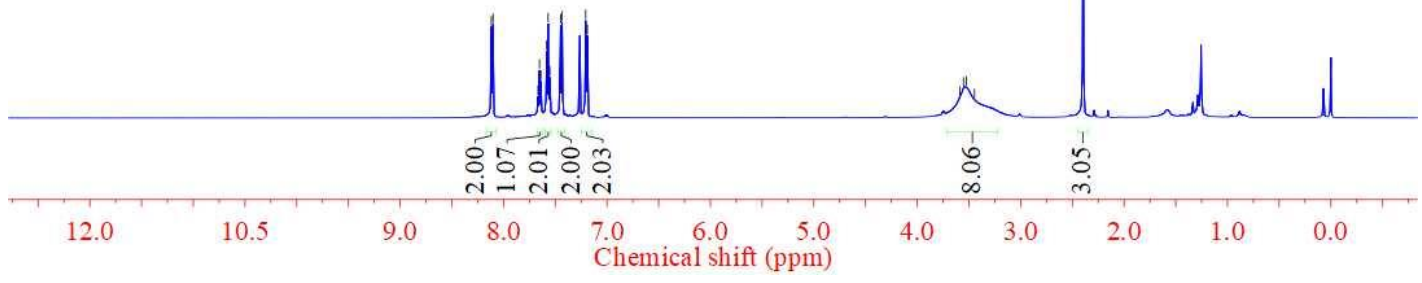

${ }^{1} \mathrm{H}$ NMR Spectrum of Compound $5 \mathrm{~b}$ 


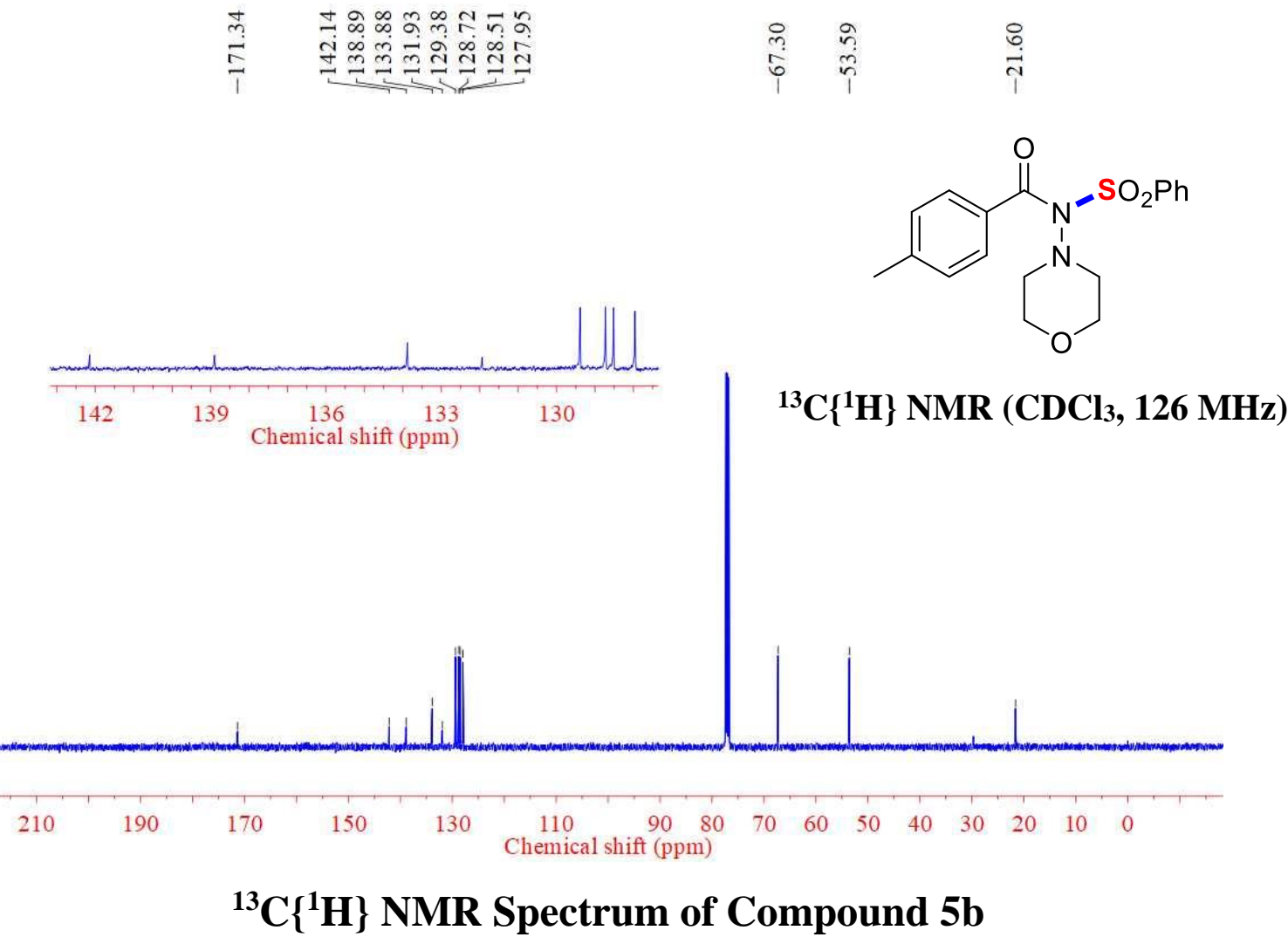

\section{Mass Spectrum SmartFormula Report}

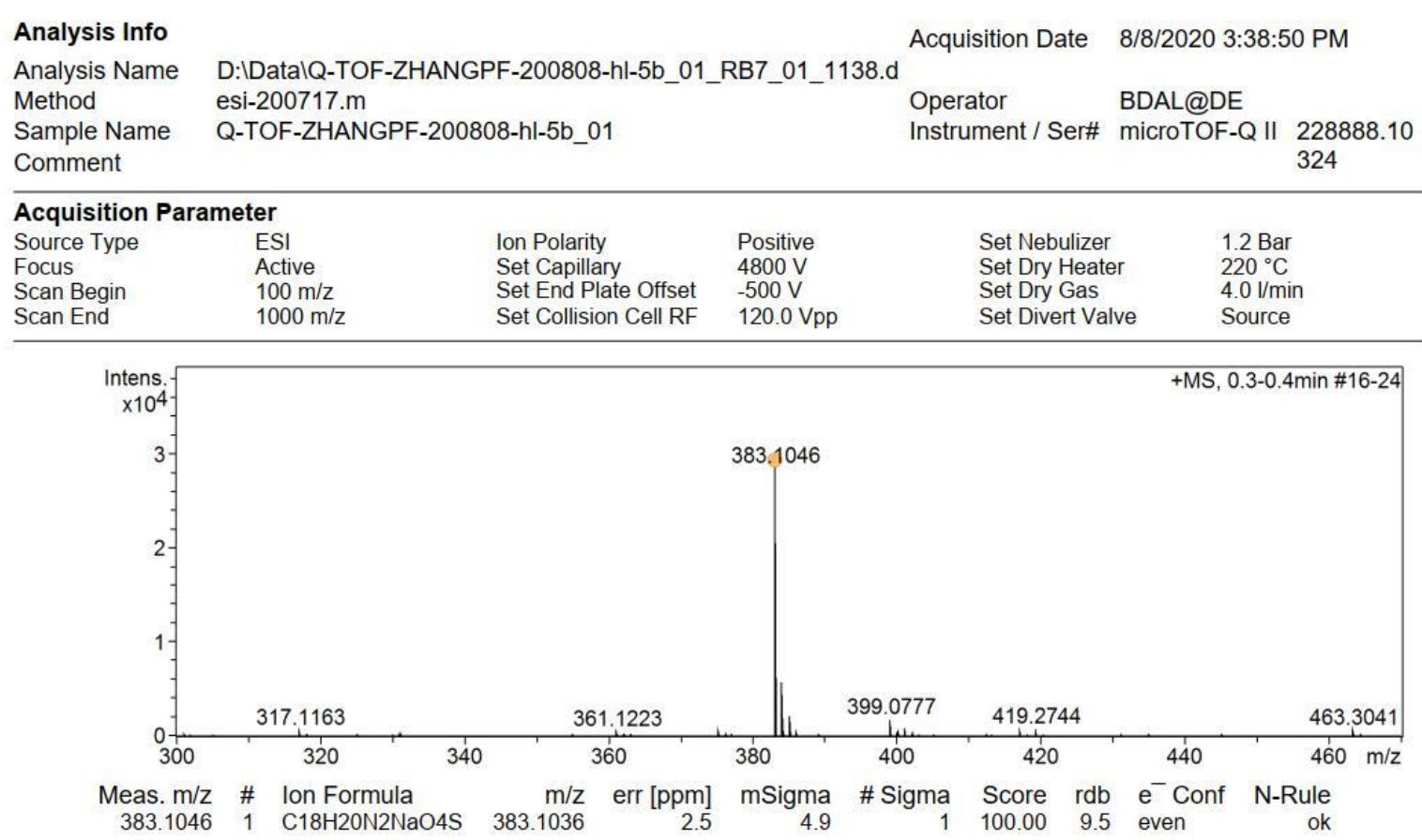

HRMS Spectrum of Compound 5b 


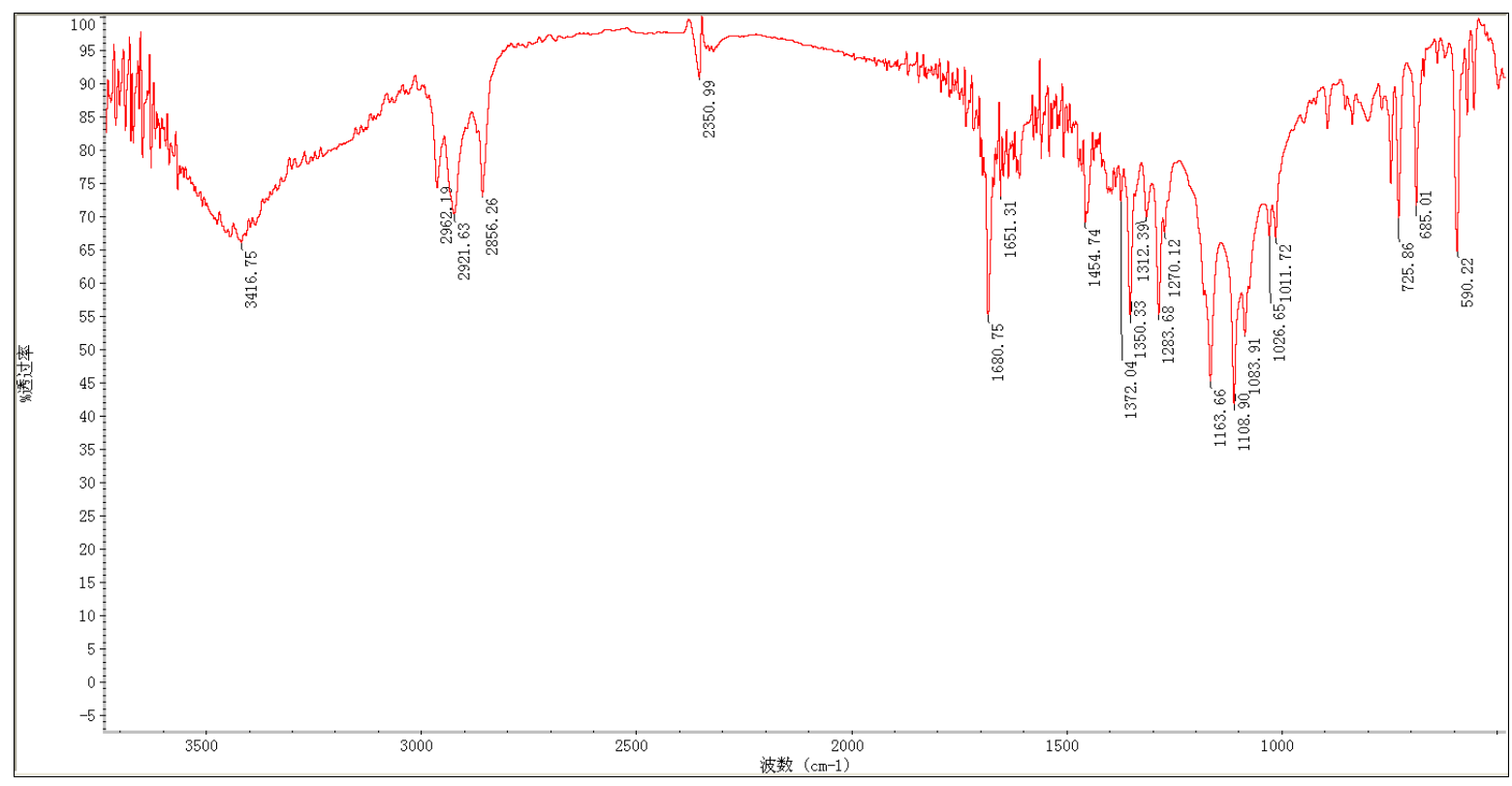

IR Spectrum of Compound $5 b$

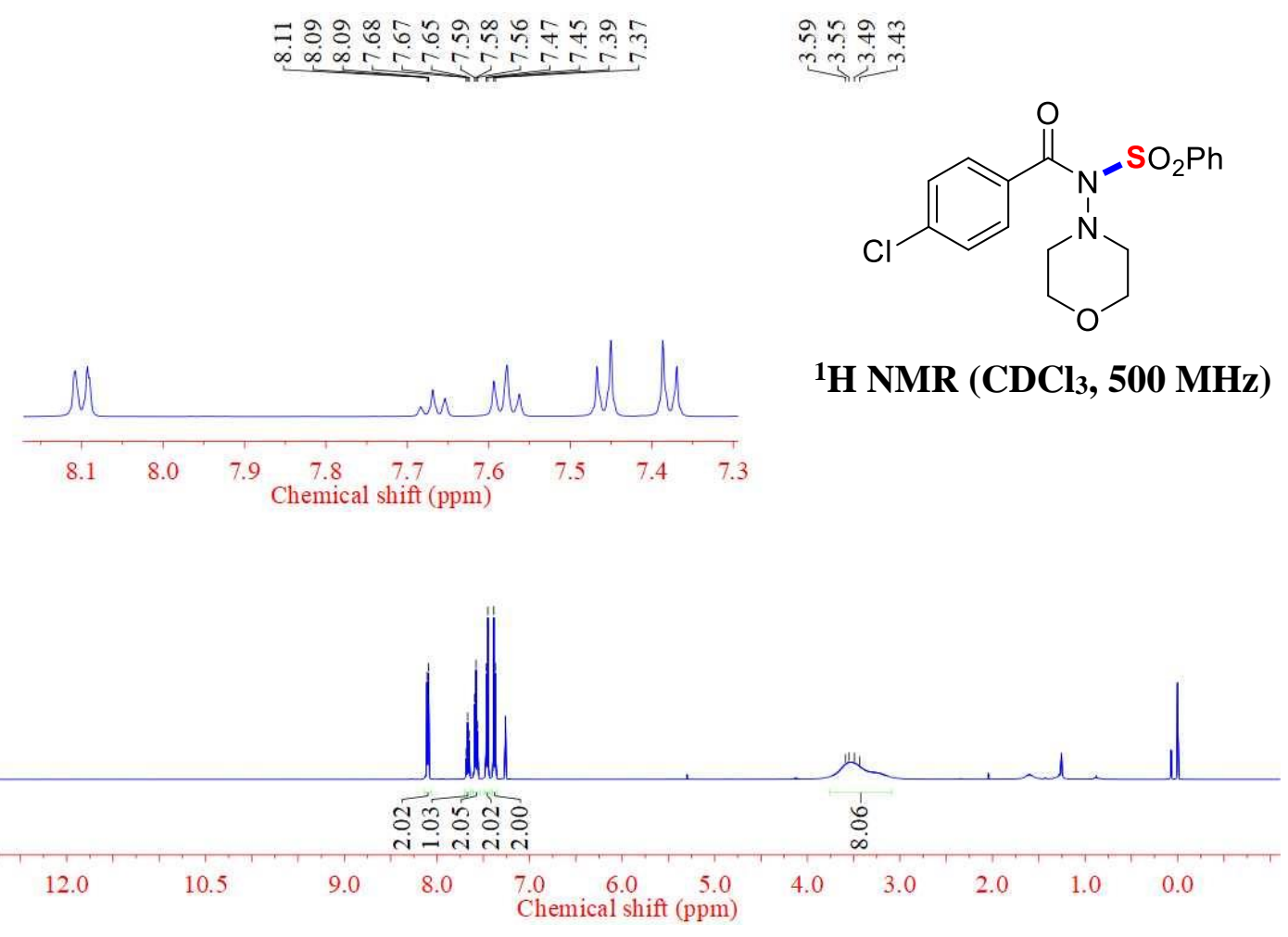

${ }^{1} \mathrm{H}$ NMR Spectrum of Compound 5c 


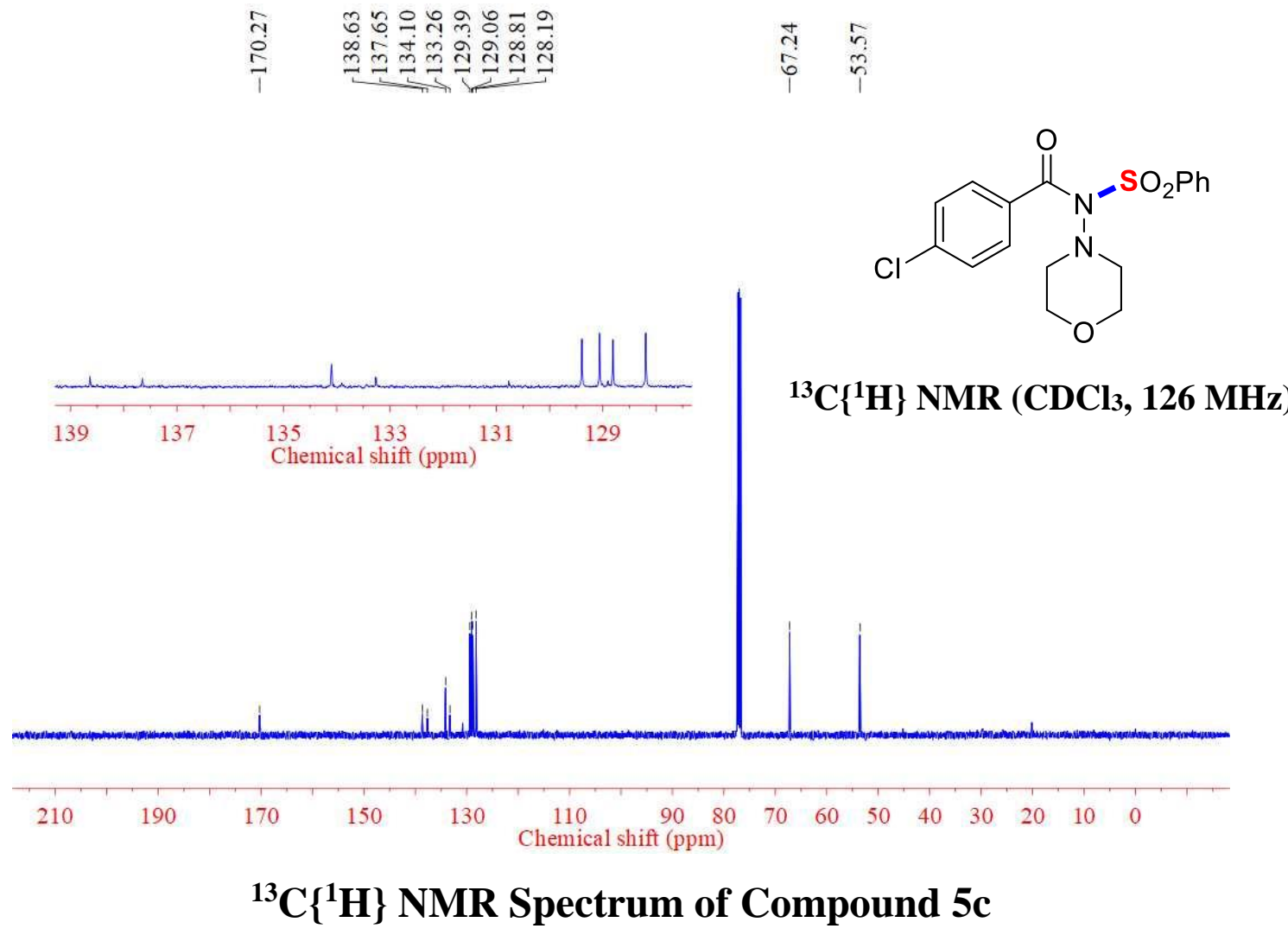

\section{Mass Spectrum SmartFormula Report}

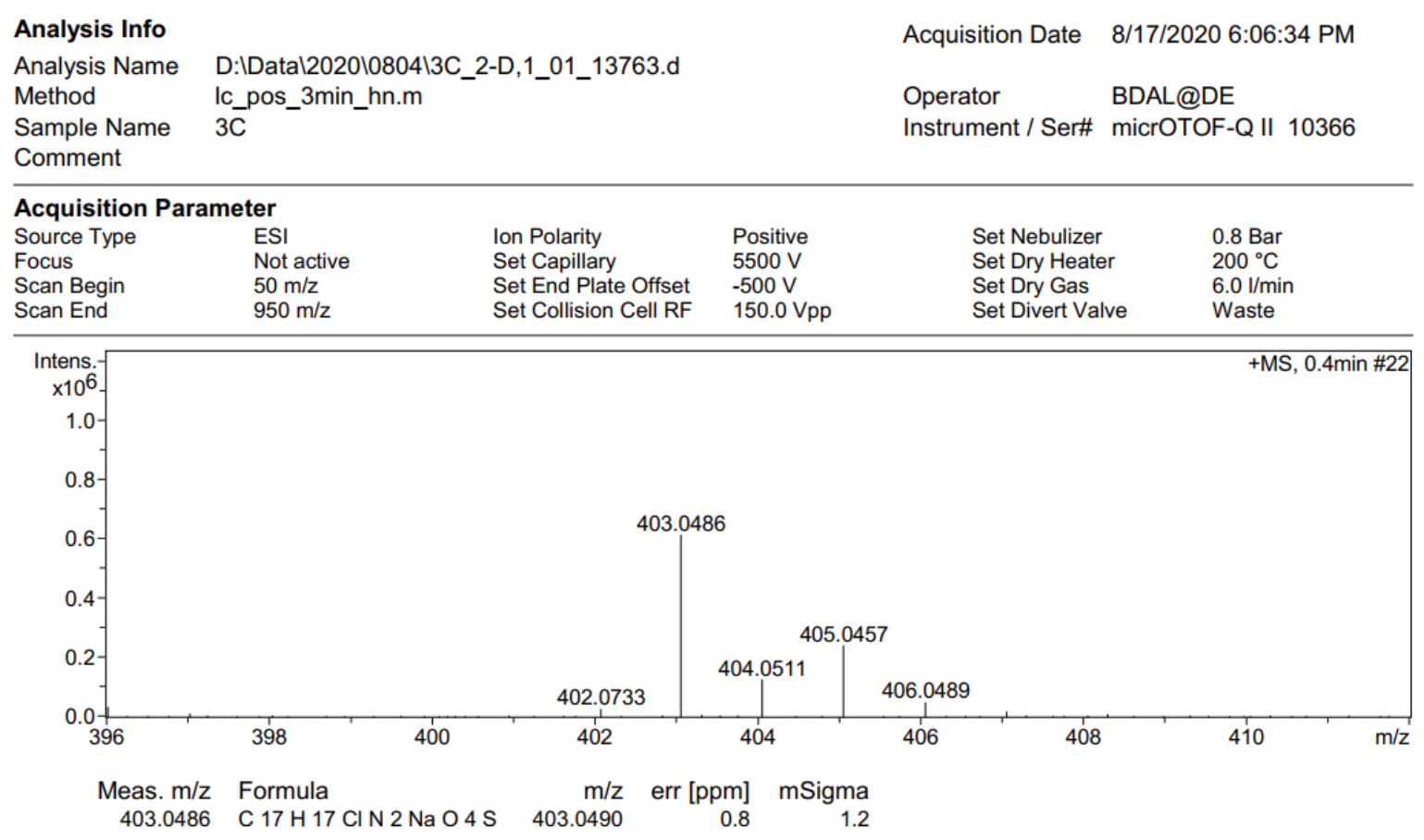

HRMS Spectrum of Compound 5c 


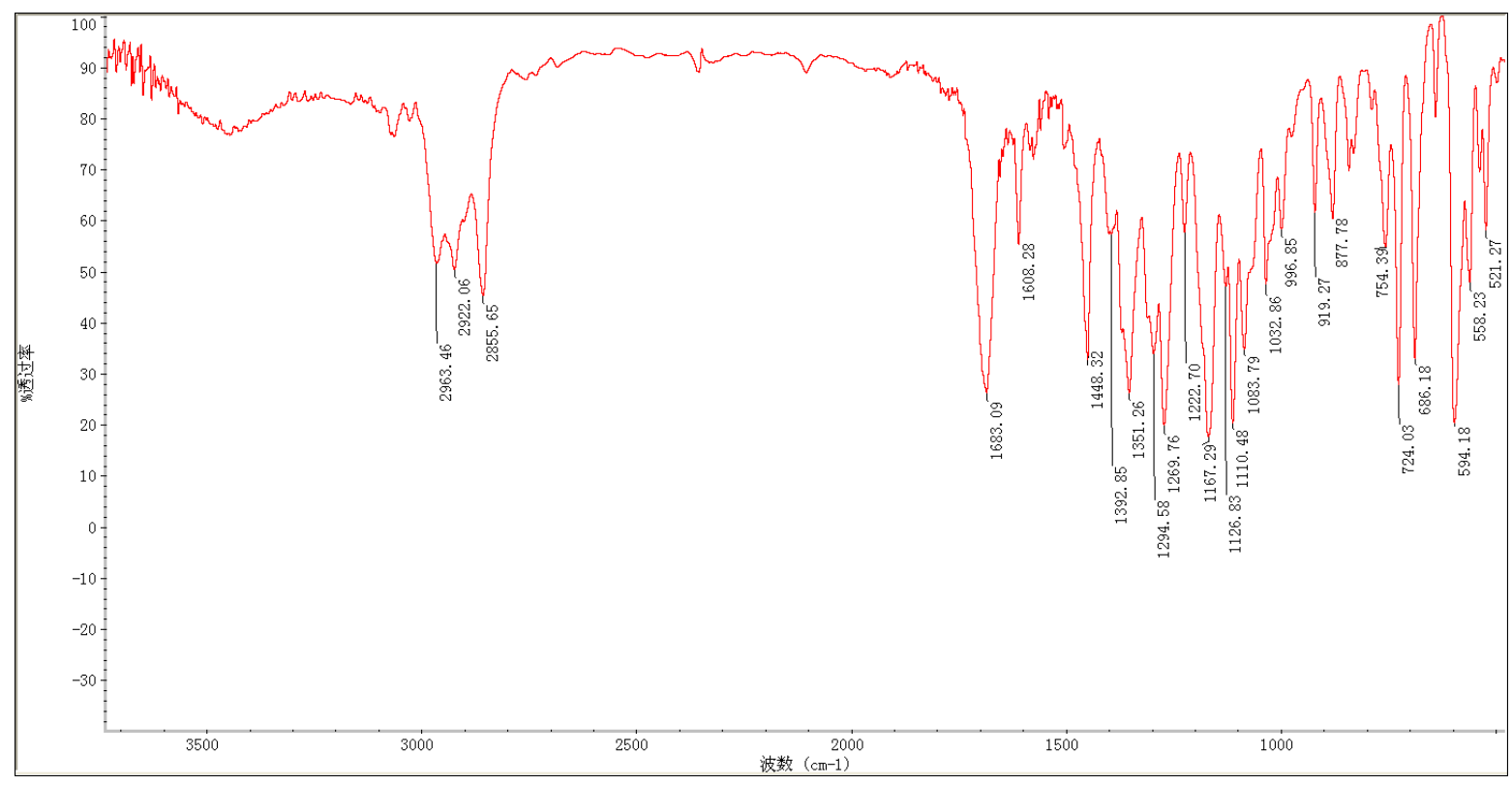

IR Spectrum of Compound $5 \mathrm{c}$<smiles>O=C(c1ccc(Br)cc1)N(N1CCOCC1)S(=O)(=O)c1ccccc1</smiles>

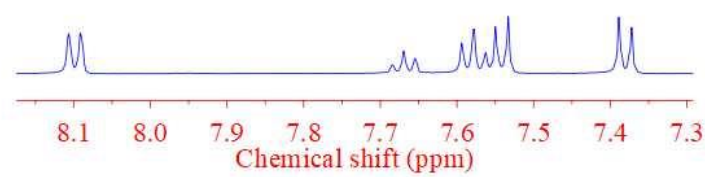

${ }^{1} \mathrm{H}$ NMR (CDCl3, $\left.500 \mathrm{MHz}\right)$

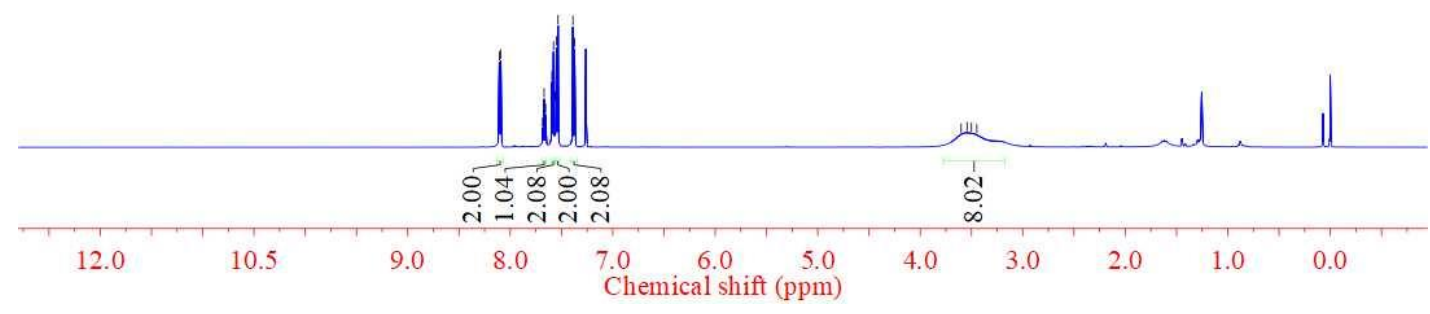

${ }^{1} \mathrm{H}$ NMR Spectrum of Compound 5d 


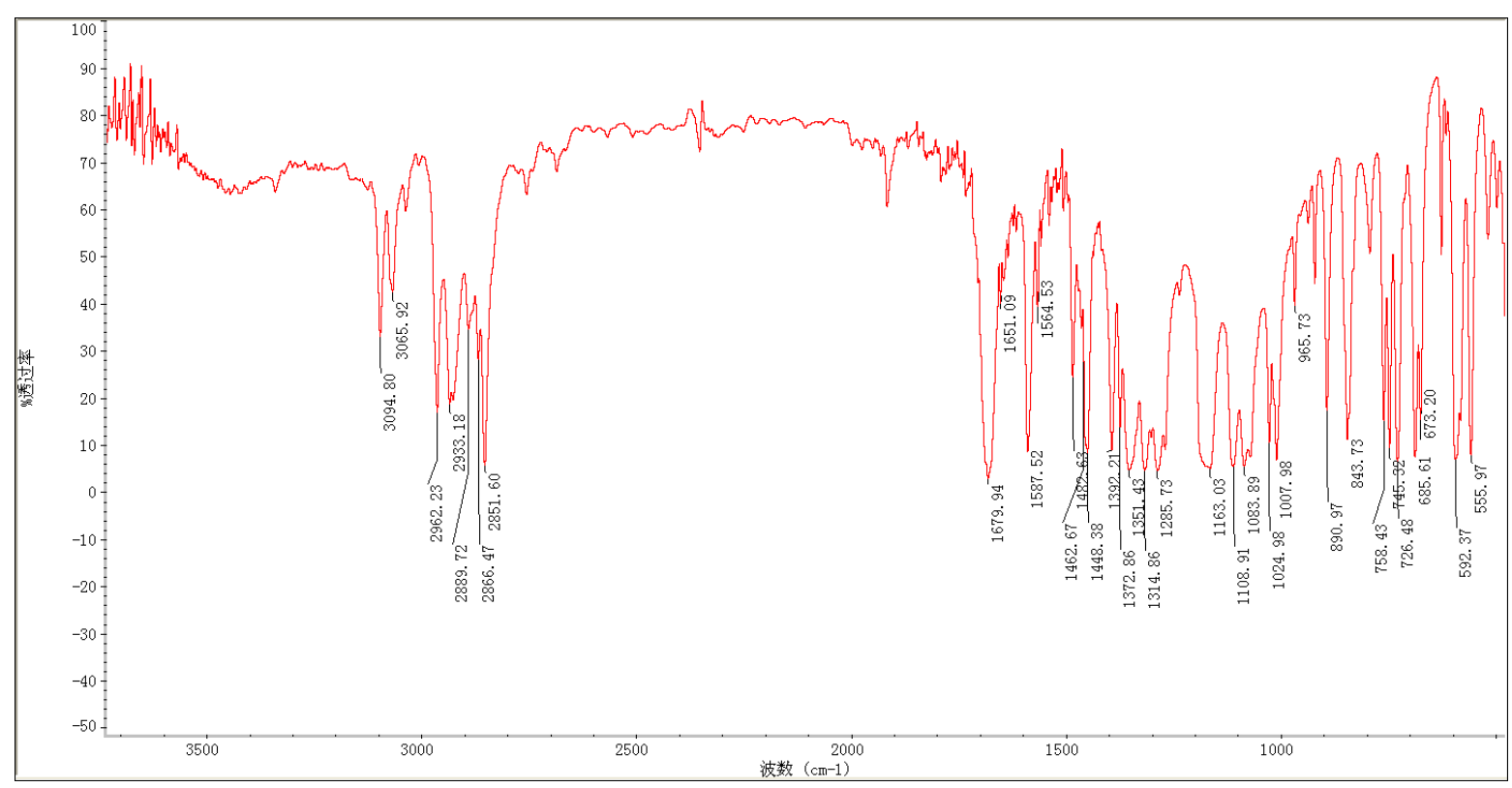

IR Spectrum of Compound 5d

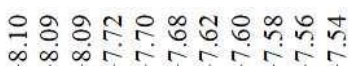

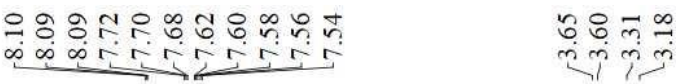

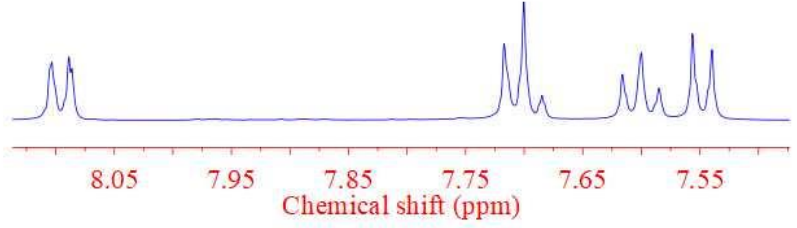<smiles>N#Cc1ccc(C(=O)N(N2CCOCC2)S(=O)(=O)c2ccccc2)cc1</smiles>

${ }^{1} \mathrm{H}$ NMR (CDCl3, $\left.500 \mathrm{MHz}\right)$

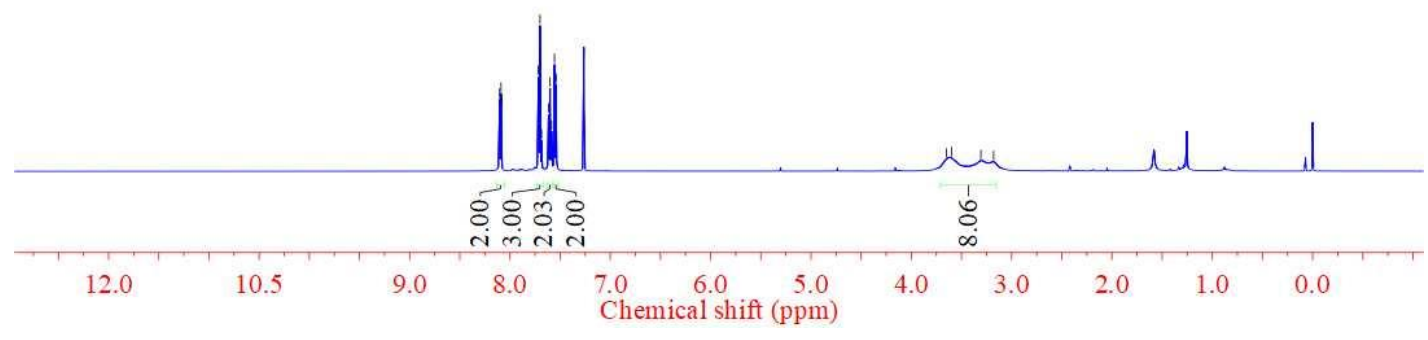

${ }^{1} \mathrm{H}$ NMR Spectrum of Compound $5 \mathrm{e}$ 


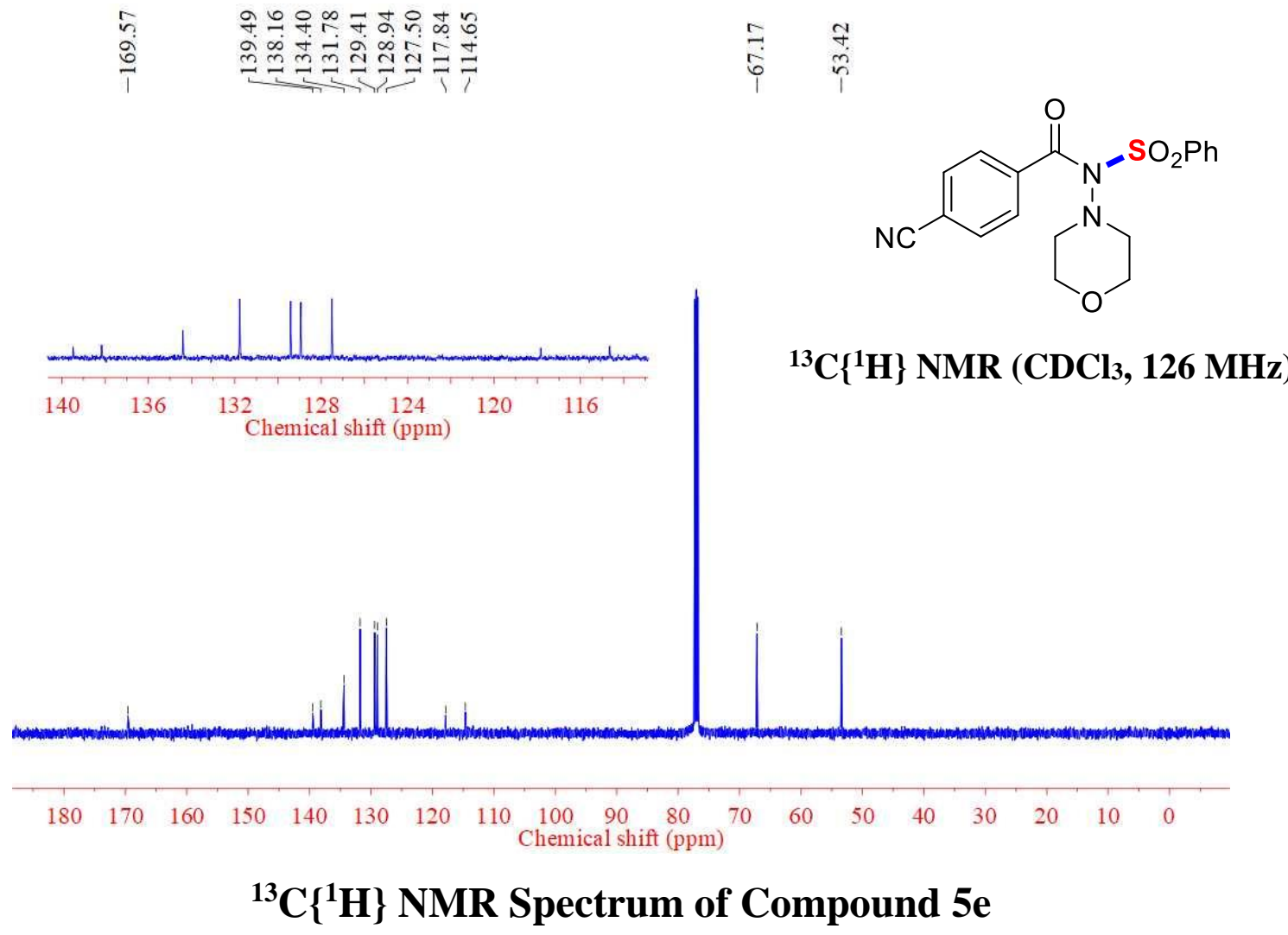

\section{Mass Spectrum SmartFormula Report}

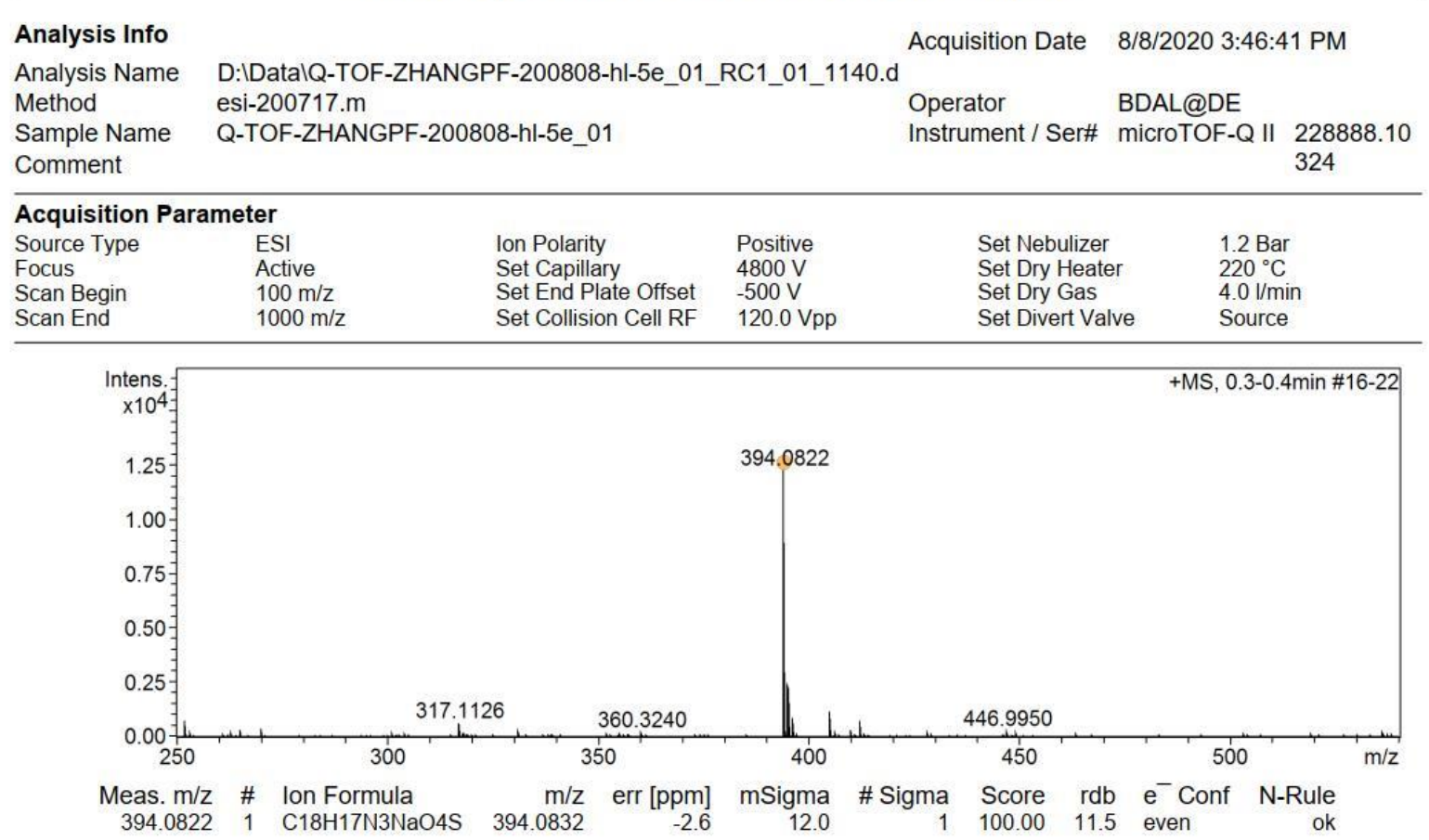

HRMS Spectrum of Compound 5e 


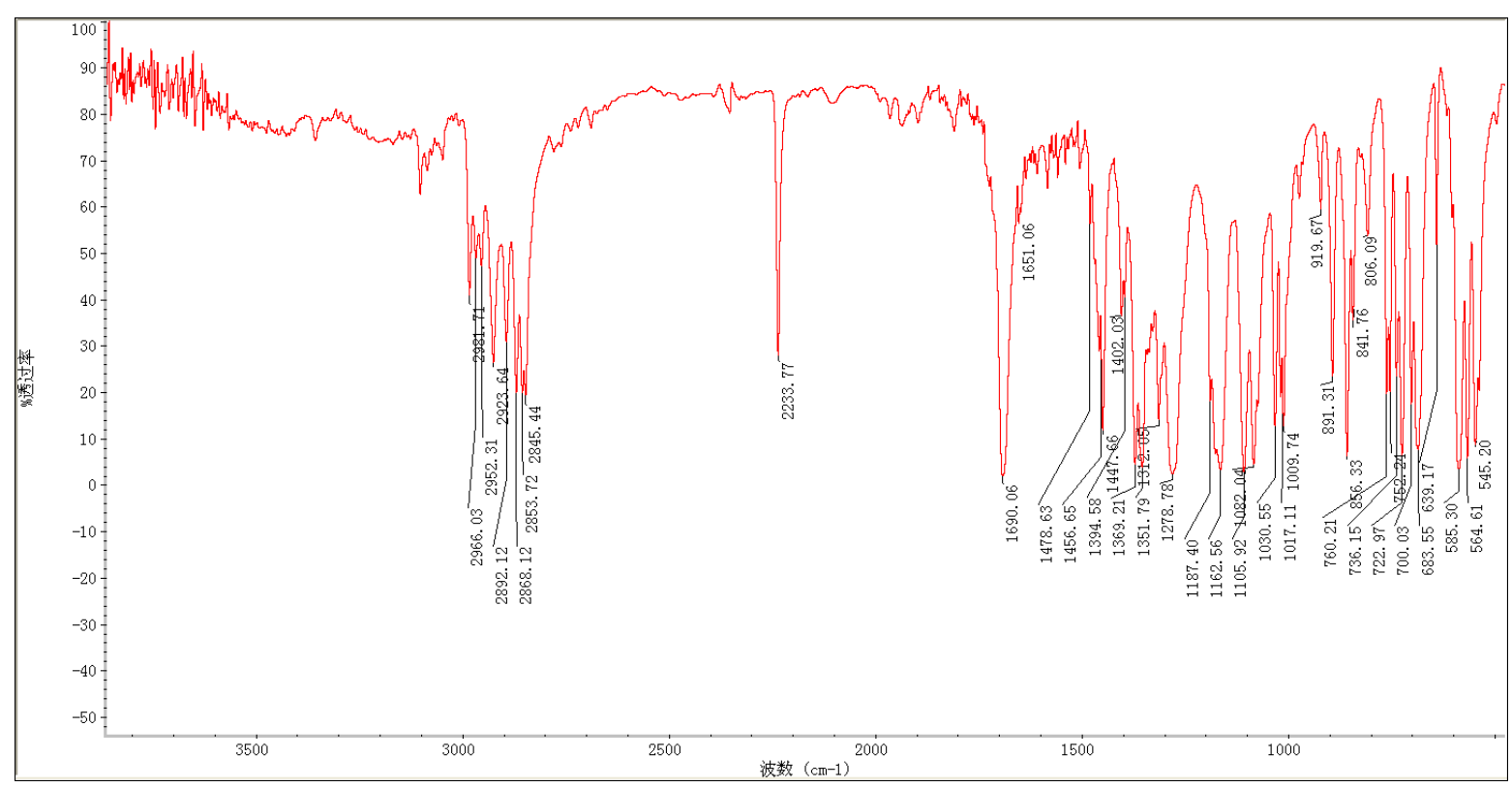

IR Spectrum of Compound $5 \mathrm{e}$

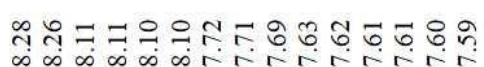

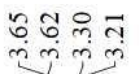<smiles>O=C(c1ccc([N+](=O)[O-])cc1)N(N1CCOCC1)S(=O)(=O)c1ccccc1</smiles>

${ }^{1} \mathrm{H}$ NMR (CDCl 3 , $\left.500 \mathrm{MHz}\right)$
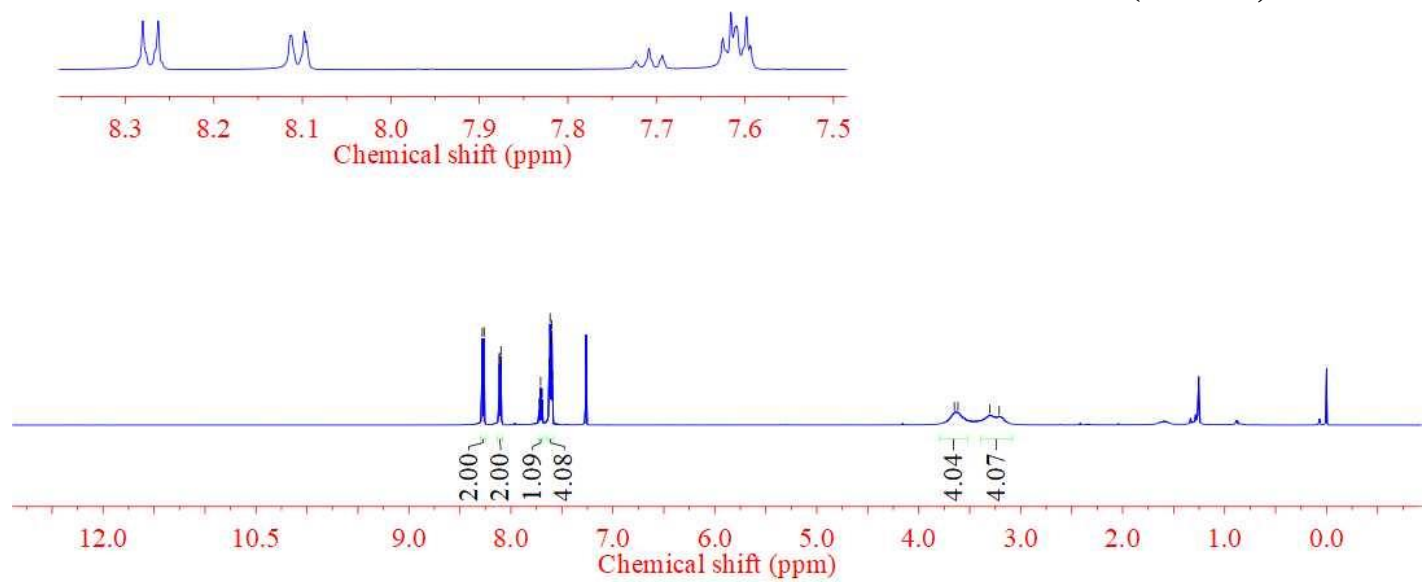

${ }^{1}$ H NMR Spectrum of Compound $5 f$ 


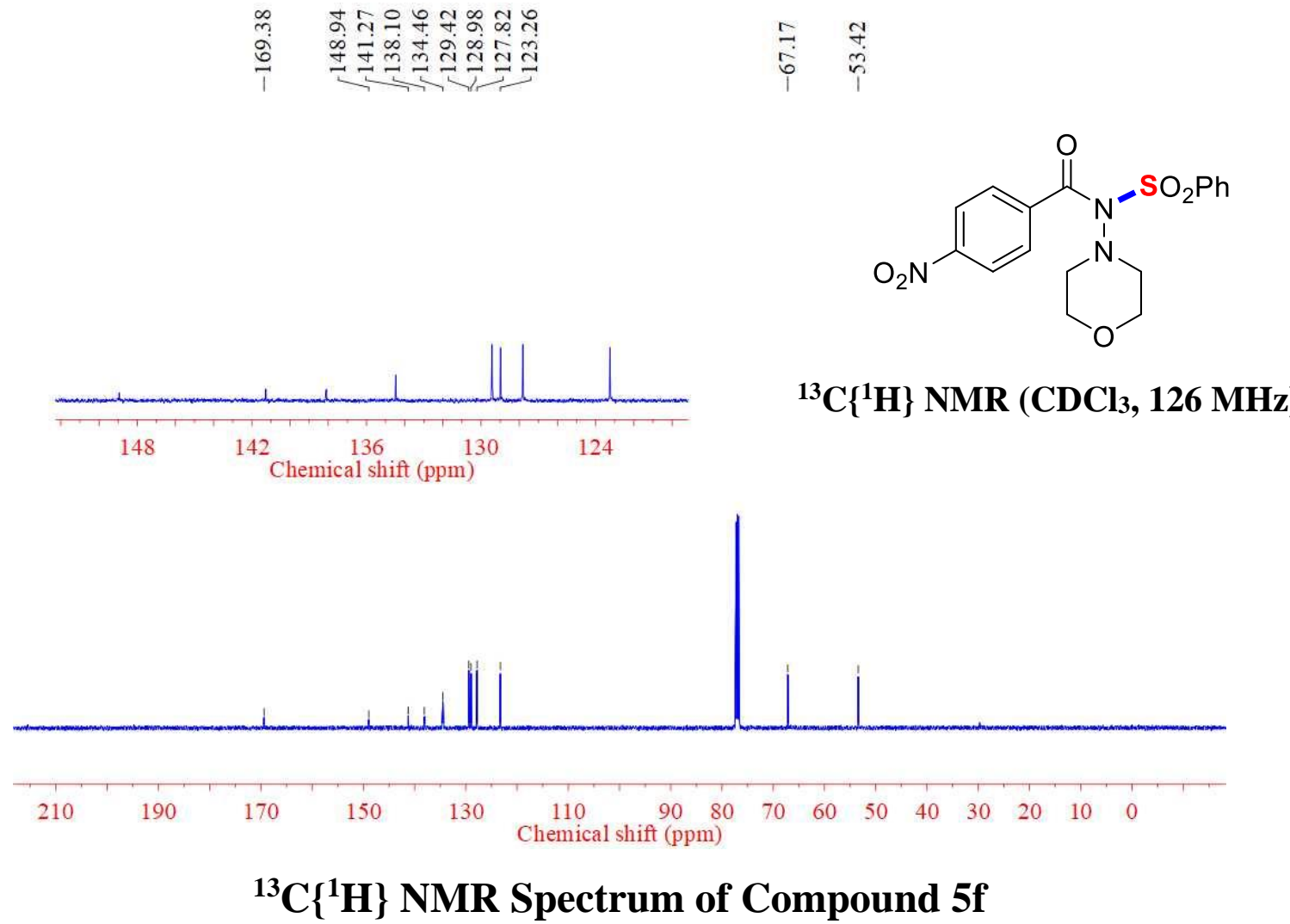

\begin{tabular}{|c|c|c|c|c|c|c|}
\hline \multicolumn{7}{|c|}{ Mass Spectrum SmartFormula Report } \\
\hline $\begin{array}{l}\text { Analysis Info } \\
\text { Analysis Name } \\
\text { Method } \\
\text { Sample Name } \\
\text { Comment }\end{array}$ & \multicolumn{3}{|c|}{$\begin{array}{l}\text { D:IDatalQ-TOF-ZHANGPF-200808-hl-5f_01_RC2_01_1141.d } \\
\text { esi-200717.m } \\
\text { Q-TOF-ZHANGPF-200808-hl-5f_01 }\end{array}$} & $\begin{array}{l}\text { Acquisition Date } \\
\text { Operator } \\
\text { Instrument / Ser\# }\end{array}$ & \multicolumn{2}{|c|}{ 8/8/2020 3:50:35 PM } \\
\hline $\begin{array}{l}\text { Source Type } \\
\text { Focus } \\
\text { Scan Begin } \\
\text { Scan End }\end{array}$ & $\begin{array}{l}\text { ESI } \\
\text { Active } \\
100 \mathrm{~m} / \mathrm{z} \\
1000 \mathrm{~m} / \mathrm{z}\end{array}$ & $\begin{array}{l}\text { Ion Polarity } \\
\text { Set Capillary } \\
\text { Set End Plate Offset } \\
\text { Set Collision Cell RF }\end{array}$ & $\begin{array}{l}\text { Positive } \\
4800 \mathrm{~V} \\
-500 \mathrm{~V} \\
120.0 \mathrm{Vpp}\end{array}$ & $\begin{array}{l}\text { Set Dry Heater } \\
\text { Set Dry Gas }\end{array}$ & $\begin{array}{l}1.2 \mathrm{Bar} \\
220^{\circ} \mathrm{C} \\
4.0 \mathrm{I} / \mathrm{mi} \\
\text { Source }\end{array}$ & \\
\hline
\end{tabular}

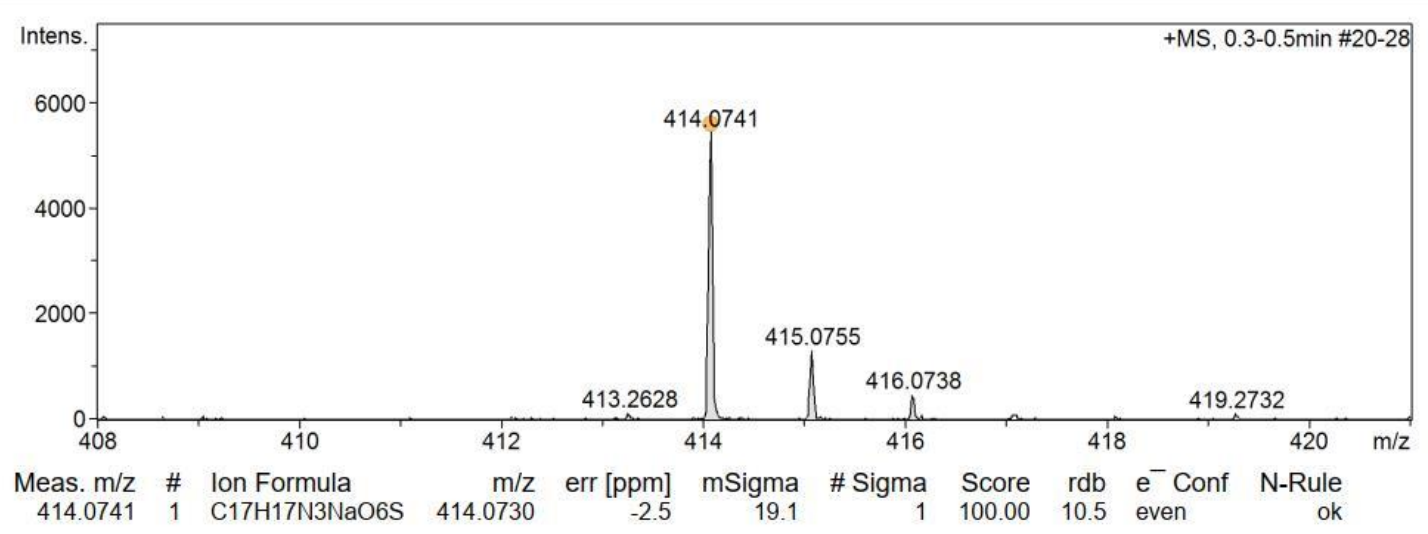

HRMS Spectrum of Compound $5 f$ 


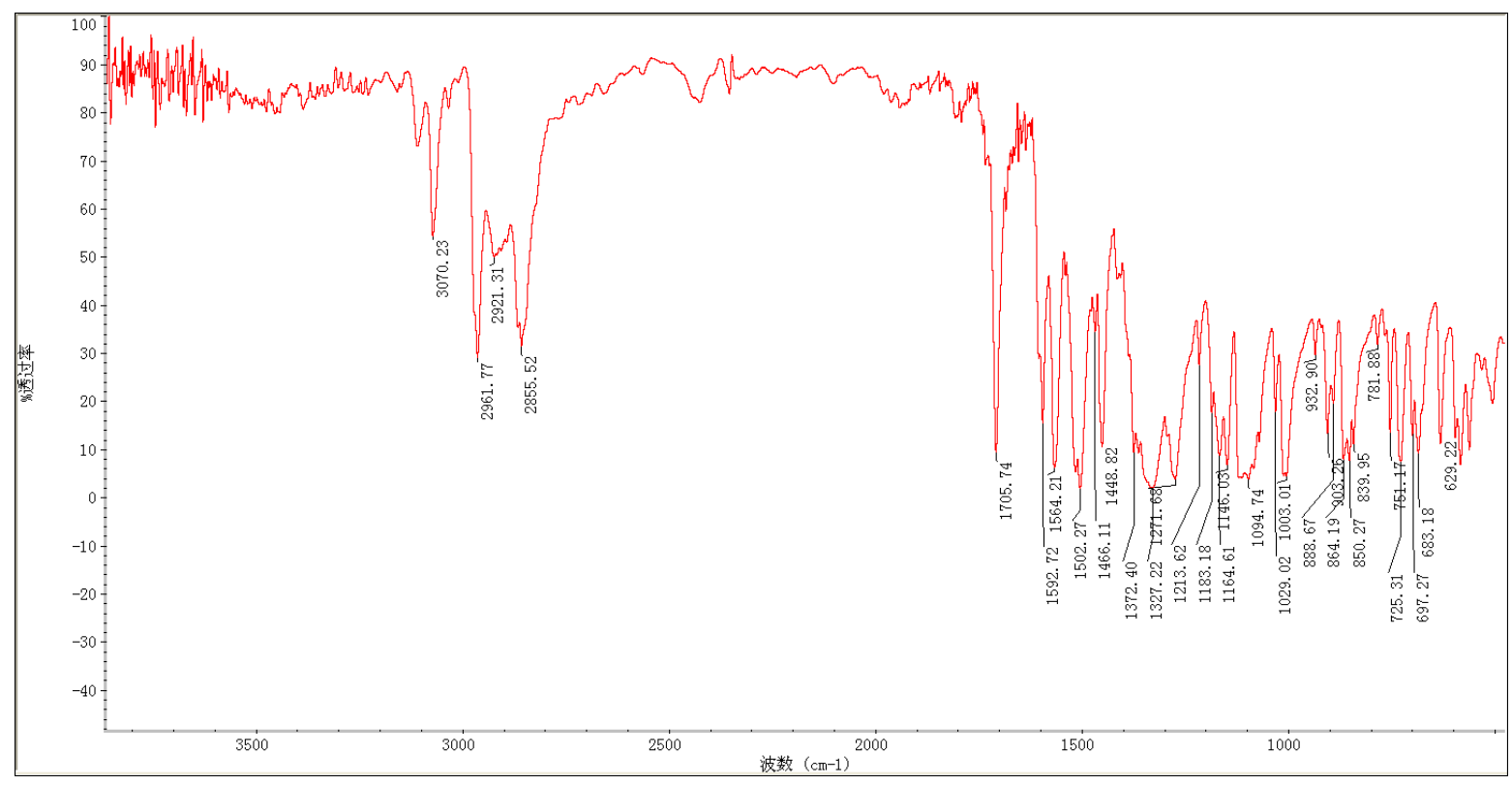

IR Spectrum of Compound $5 f$

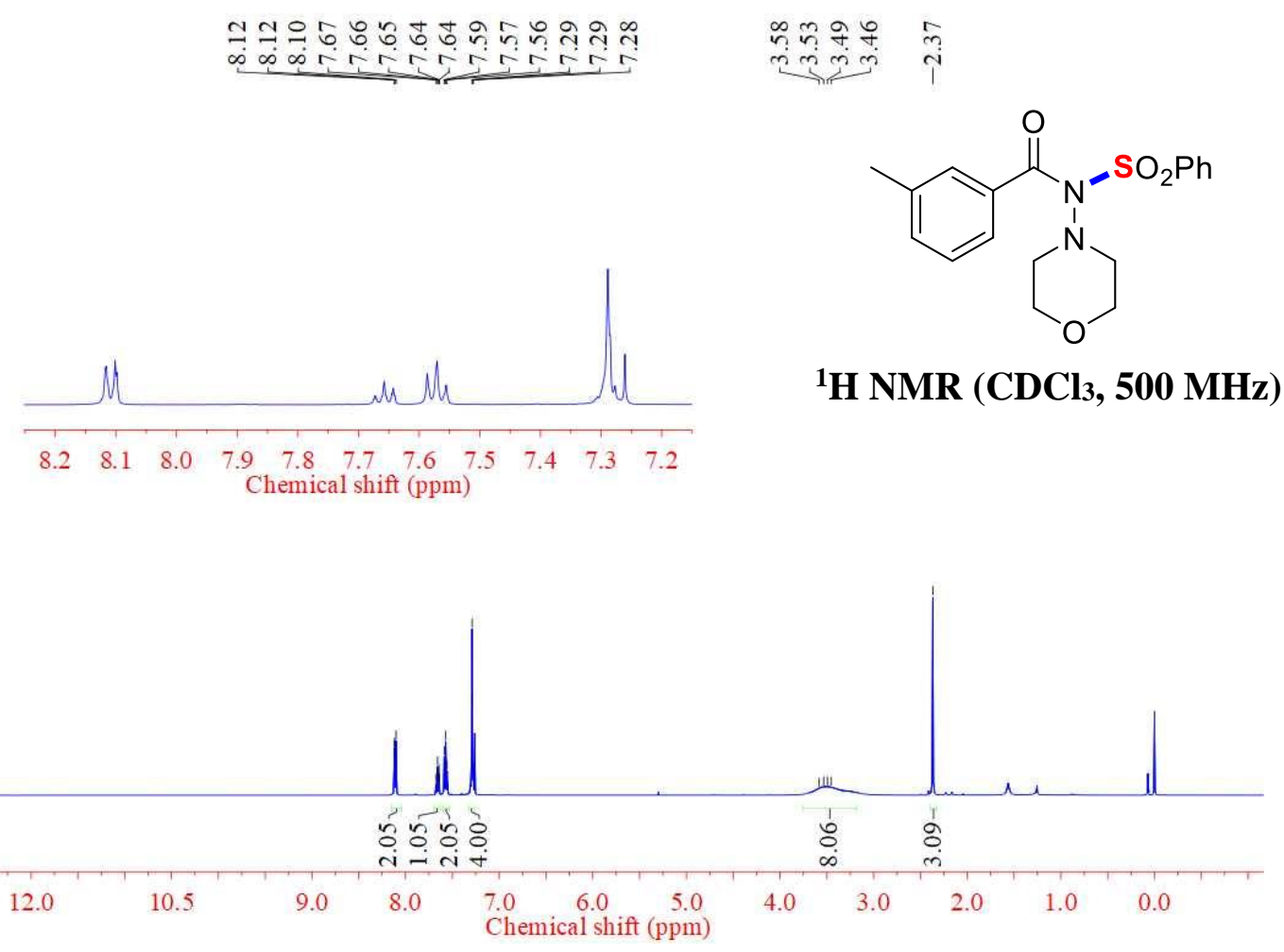

${ }^{1} \mathrm{H}$ NMR Spectrum of Compound $5 \mathrm{~g}$ 


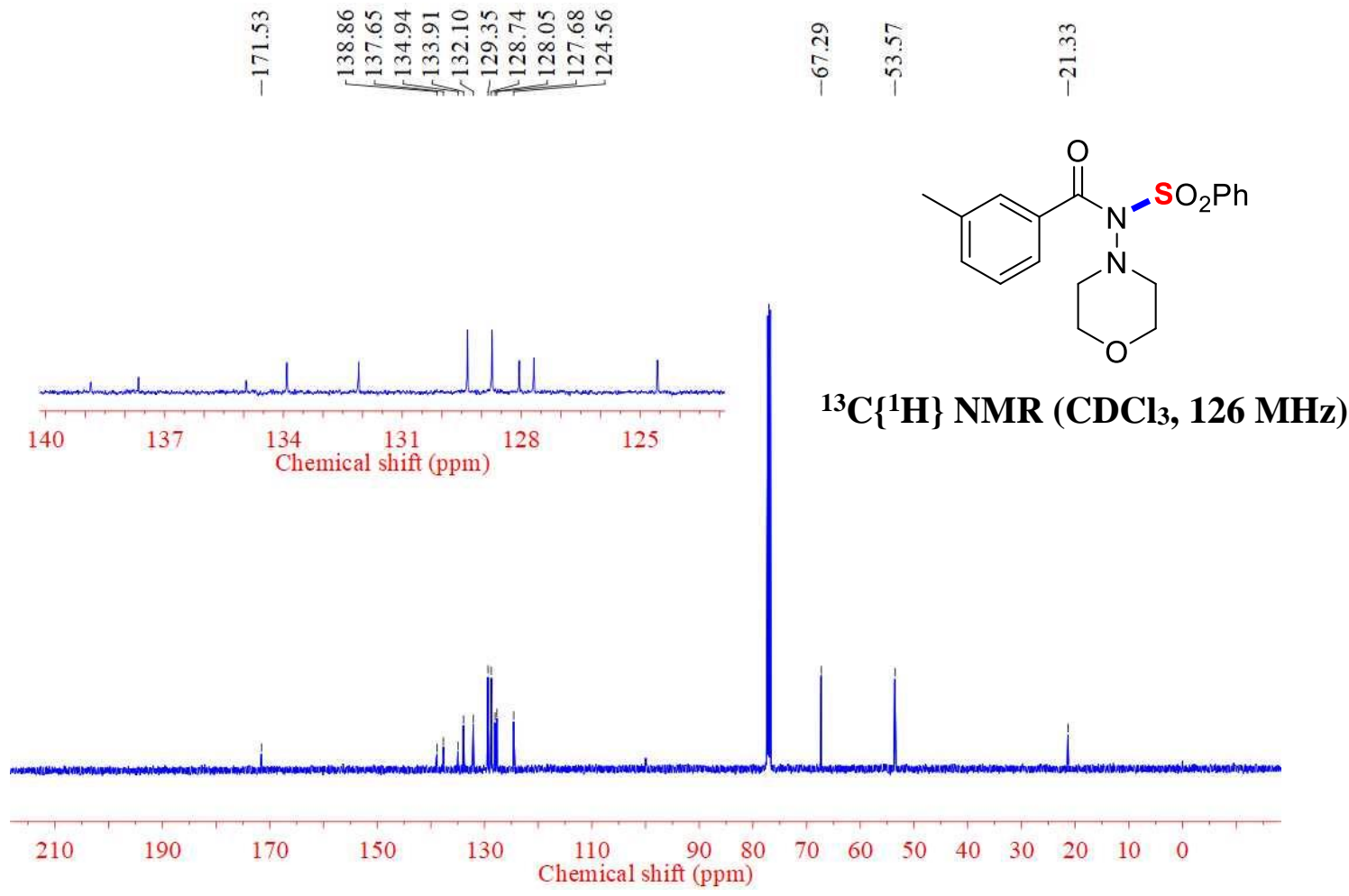

\section{${ }^{13} \mathrm{C}\left\{{ }^{1} \mathrm{H}\right\}$ NMR Spectrum of Compound $5 \mathrm{~g}$}

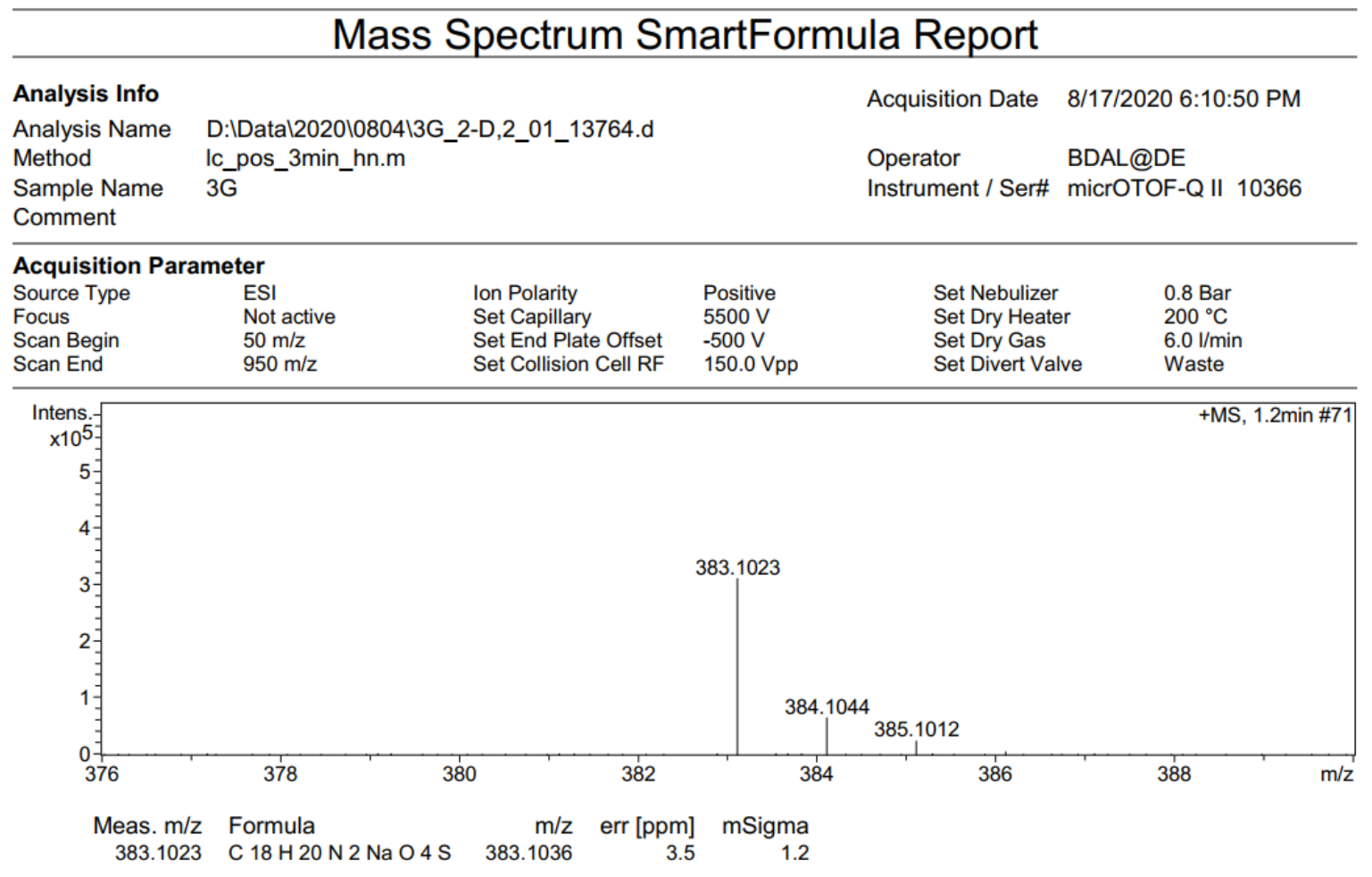

HRMS Spectrum of Compound 5g 


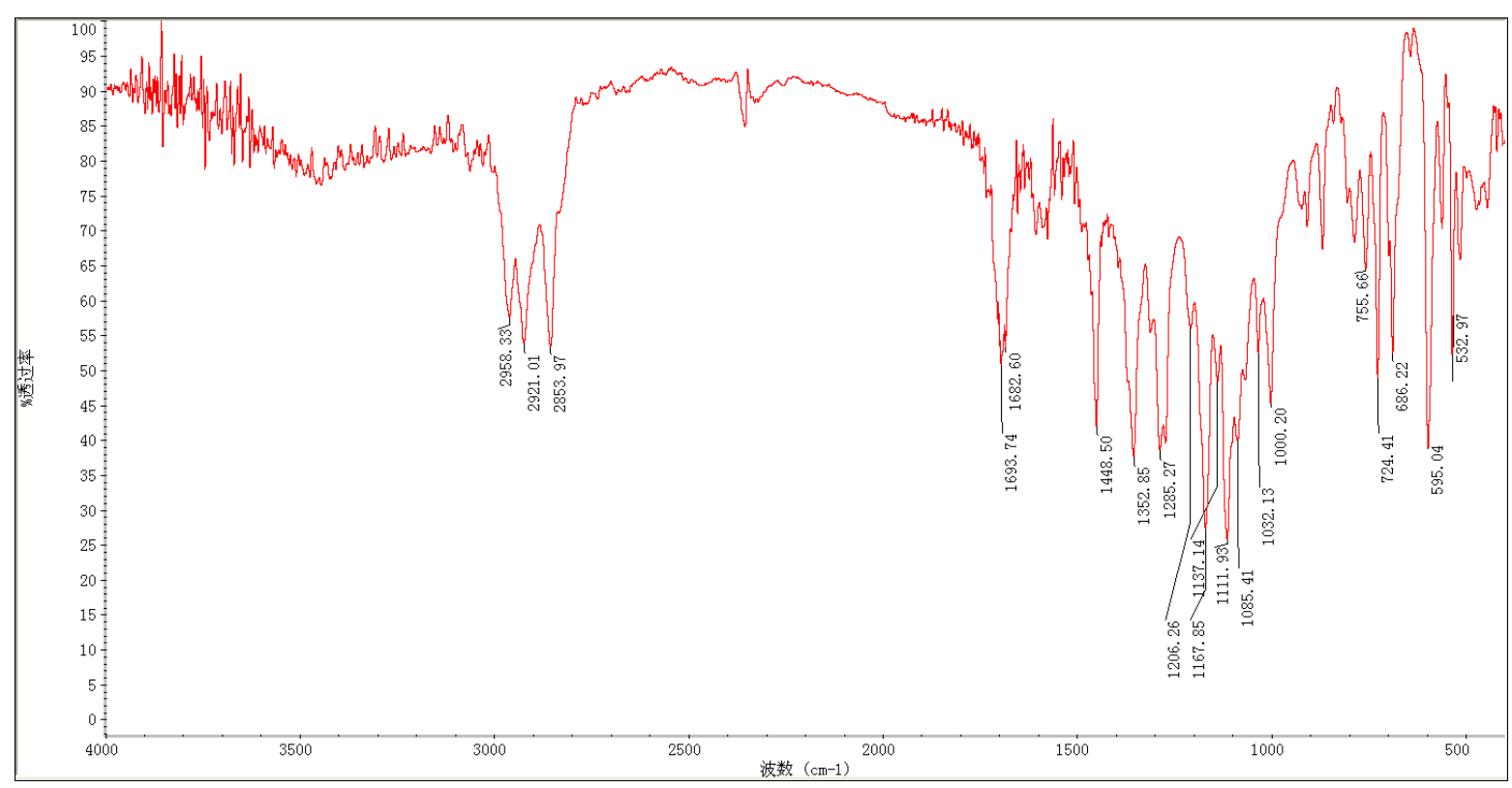

IR Spectrum of Compound 5g

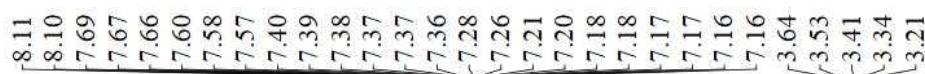<smiles>O=C(c1cccc(F)c1)N(N1CCOCC1)S(=O)(=O)c1ccccc1</smiles>

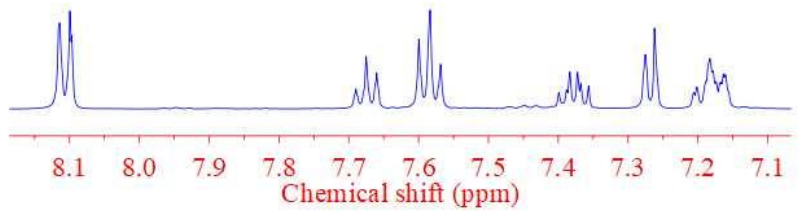

${ }^{1} \mathrm{H}$ NMR $\left(\mathrm{CDCl}_{3}, 500 \mathrm{MHz}\right)$

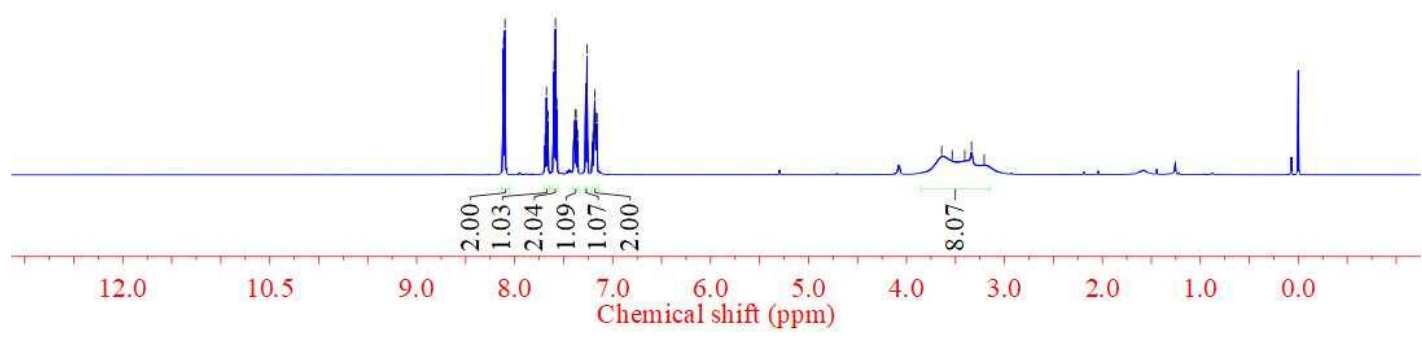

${ }^{1} \mathrm{H}$ NMR Spectrum of Compound $5 \mathrm{~h}$ 

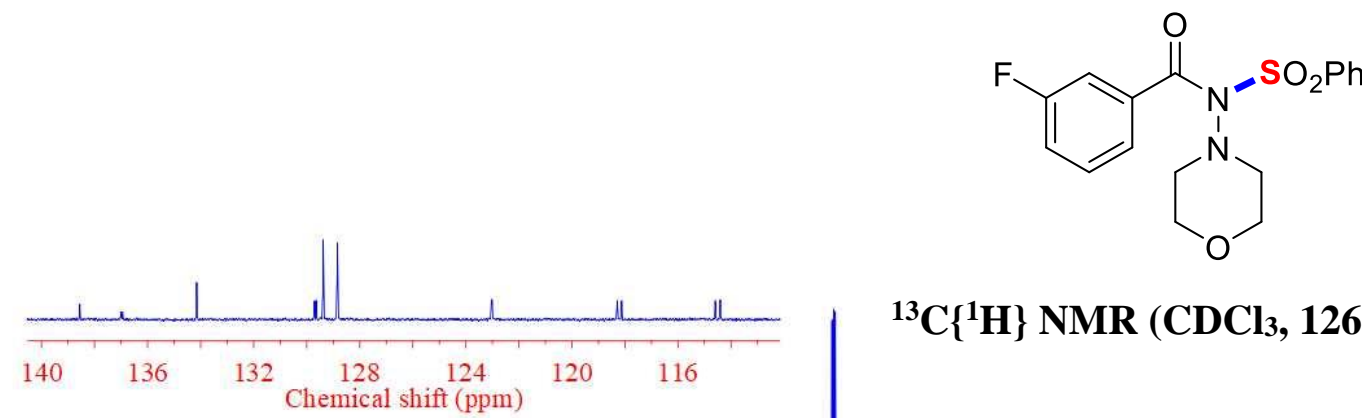

${ }^{13} \mathrm{C}\left\{{ }^{1} \mathrm{H}\right\} \mathrm{NMR}\left(\mathrm{CDCl}_{3}, 126 \mathrm{MHz}\right)$

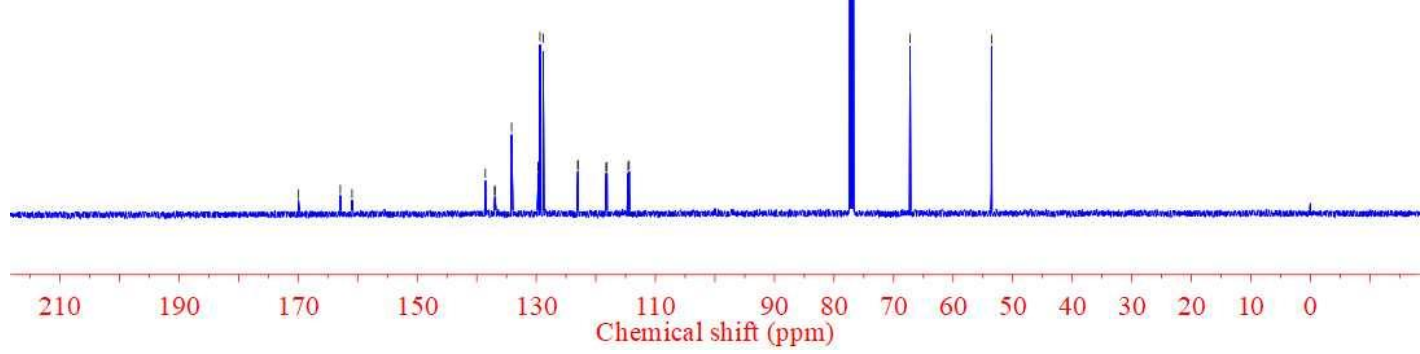

${ }^{13} \mathrm{C}\left\{{ }^{1} \mathrm{H}\right\}$ NMR Spectrum of Compound $5 \mathrm{~h}$

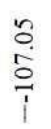<smiles>O=C(c1cccc(F)c1)N(N1CCOCC1)S(=O)(=O)c1ccccc1</smiles>

${ }^{19} \mathrm{~F}\left\{{ }^{1} \mathrm{H}\right\}$ NMR $\left(\mathrm{CDCl}_{3}, 471 \mathrm{MHz}\right)$

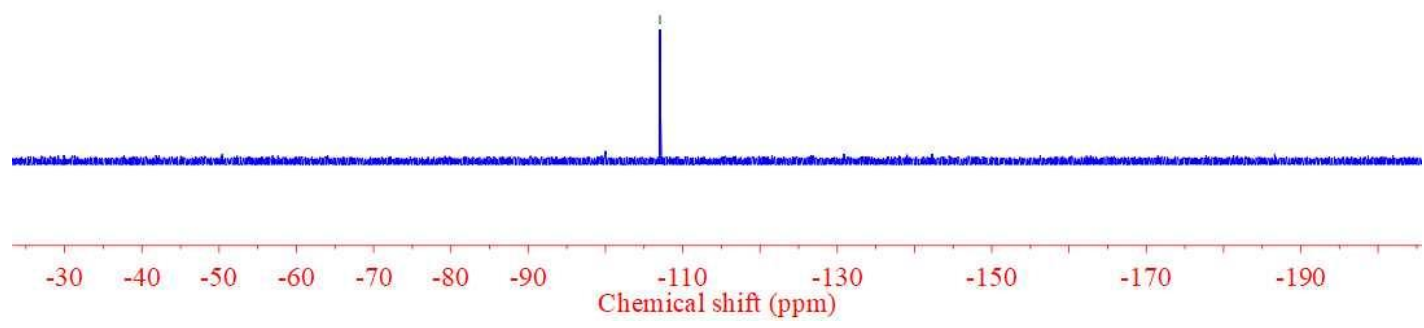

${ }^{19} \mathbf{F}\left\{{ }^{1} \mathrm{H}\right\}$ NMR Spectrum of Compound $5 h$ 


\section{Mass Spectrum SmartFormula Report}

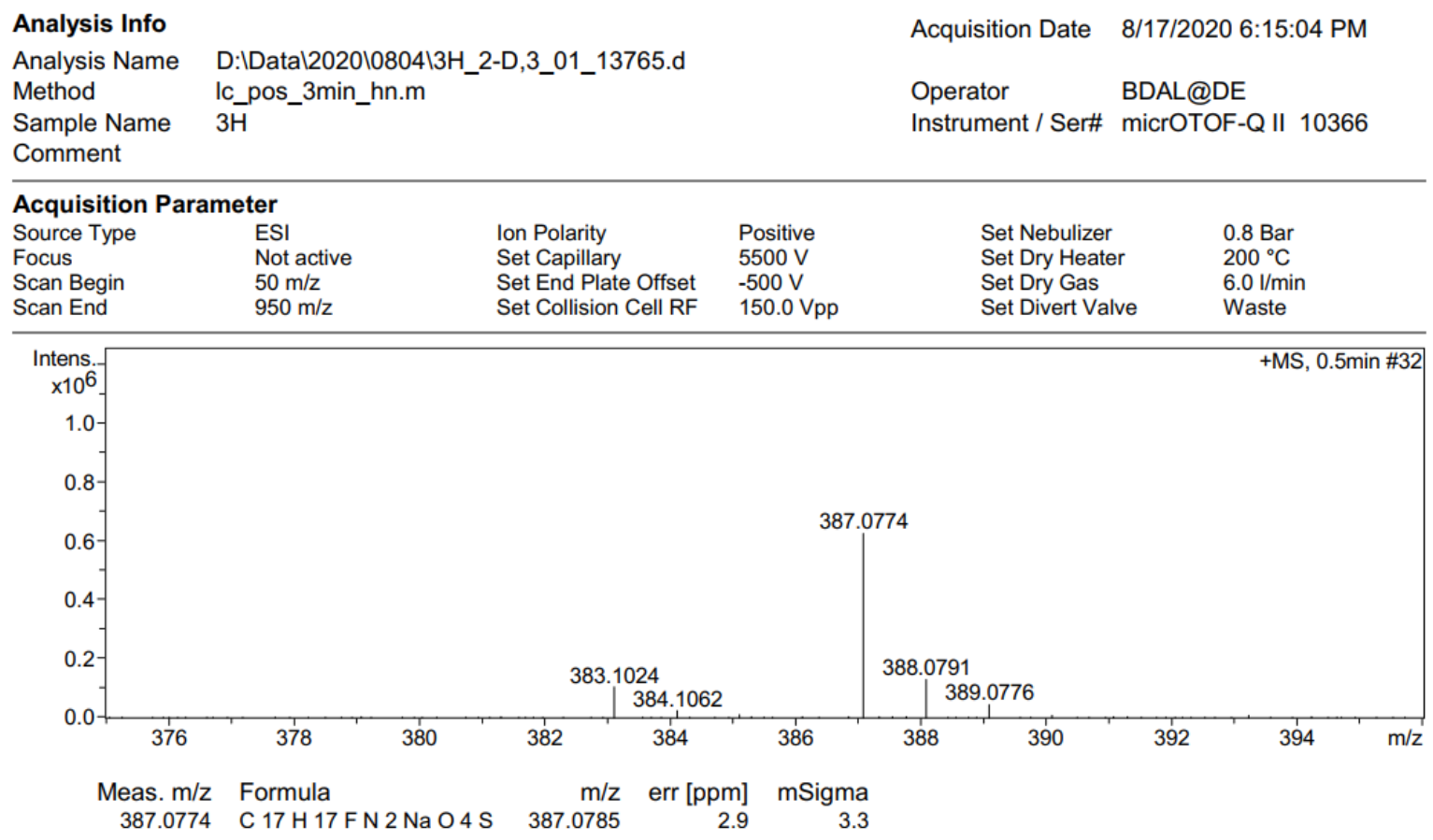

HRMS Spectrum of Compound 5h

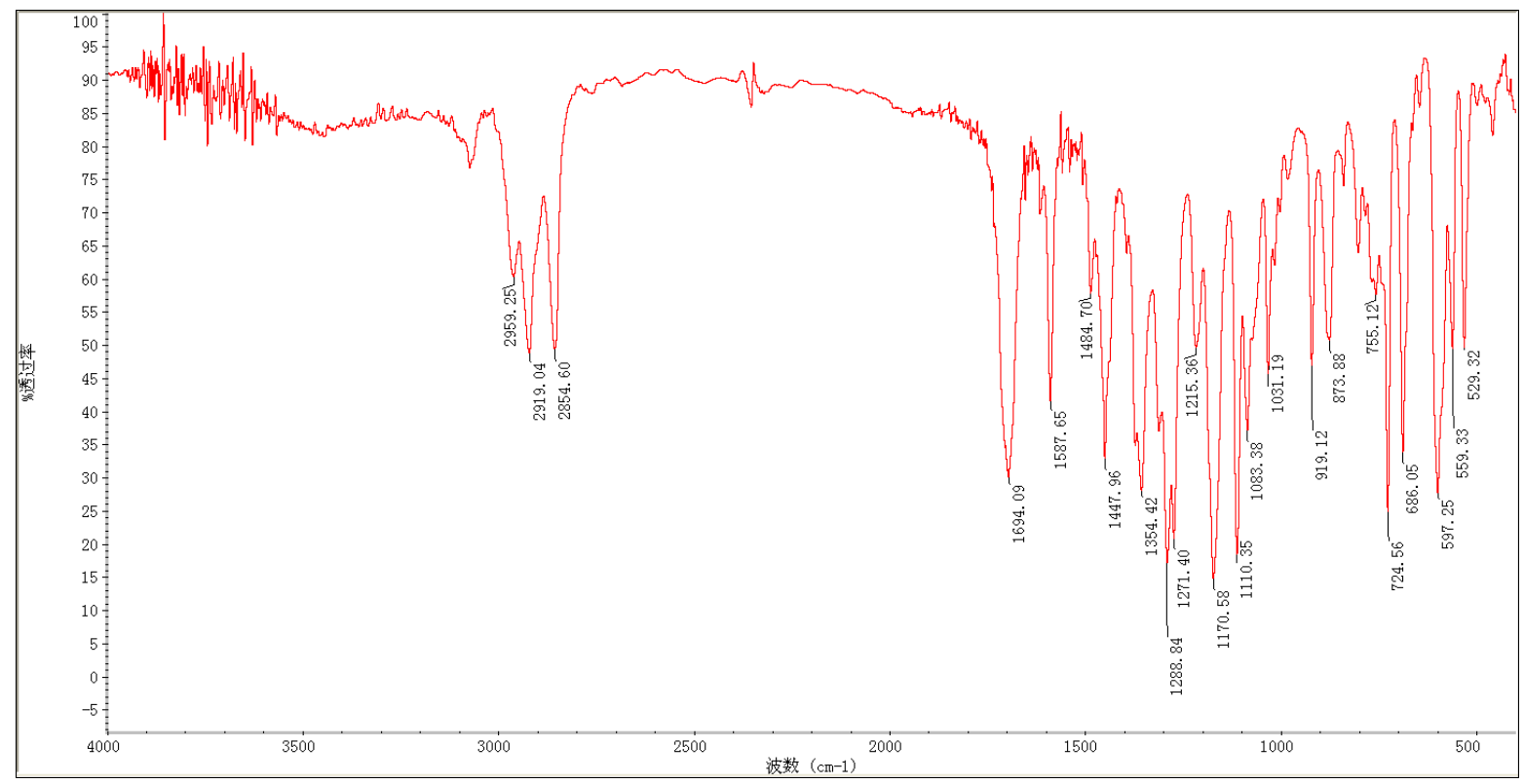

IR Spectrum of Compound 5h 


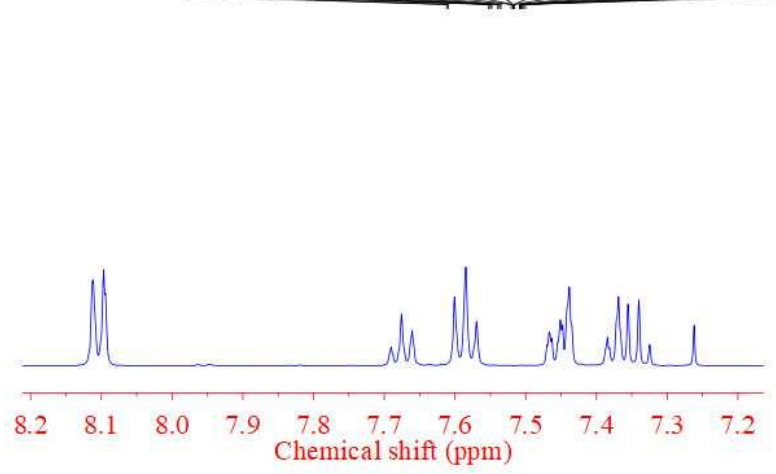<smiles>O=C(c1cccc(Cl)c1)N(N1CCOCC1)S(=O)(=O)c1ccccc1</smiles>

${ }^{1} \mathrm{H}$ NMR (CDCl3, $\left.500 \mathrm{MHz}\right)$

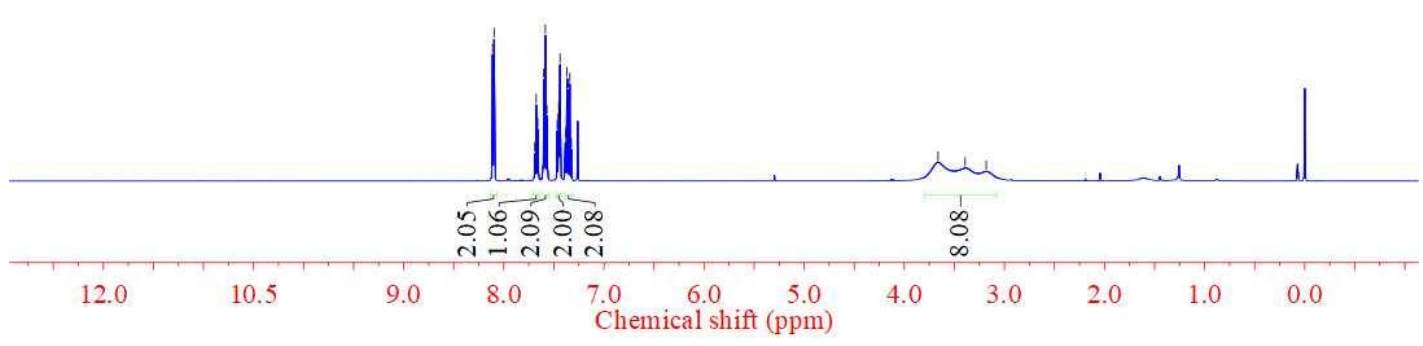

${ }^{1} \mathrm{H}$ NMR Spectrum of Compound $5 \mathrm{i}$

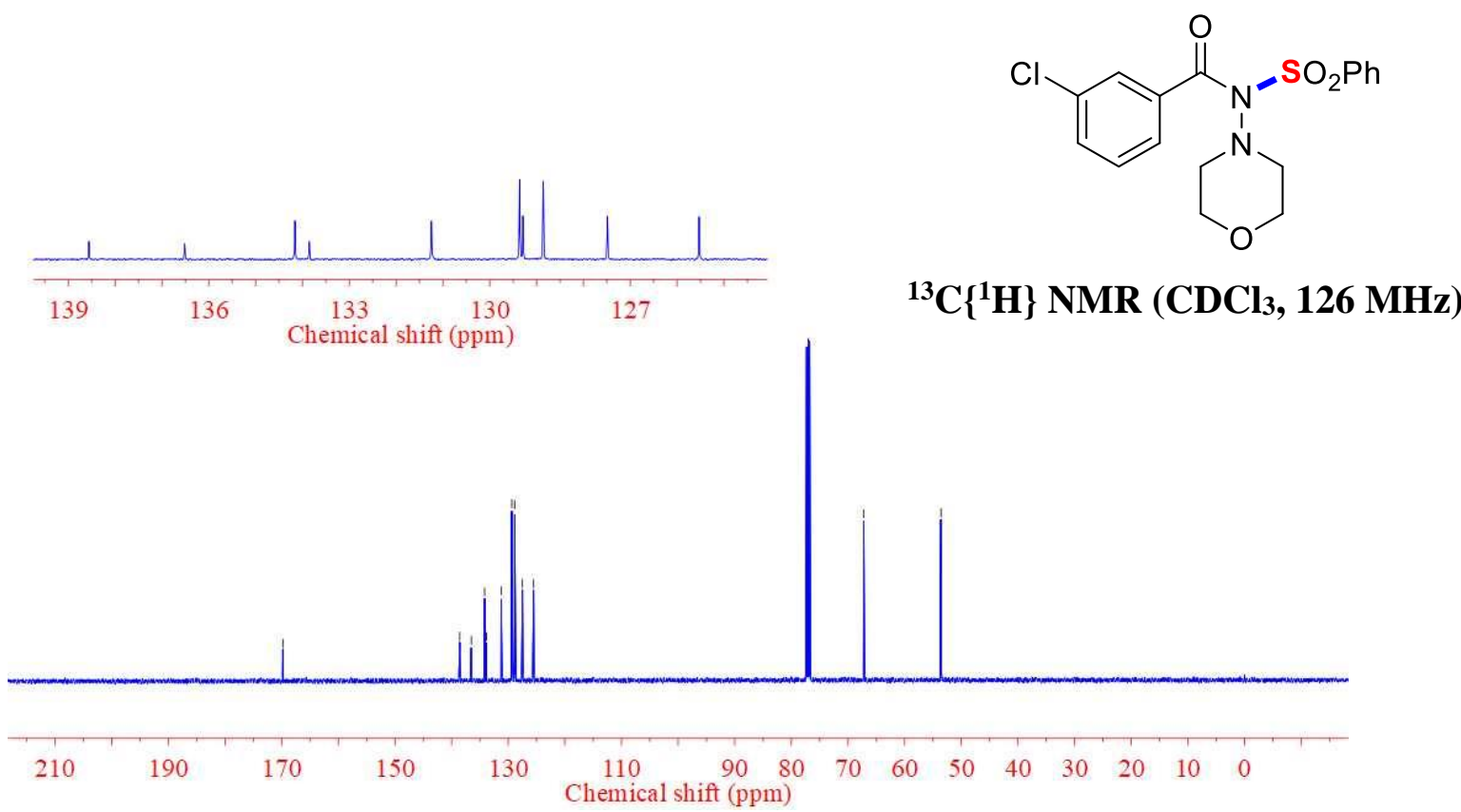

${ }^{13} \mathrm{C}\left\{{ }^{1} \mathrm{H}\right\}$ NMR Spectrum of Compound 5i 


\section{Mass Spectrum SmartFormula Report}

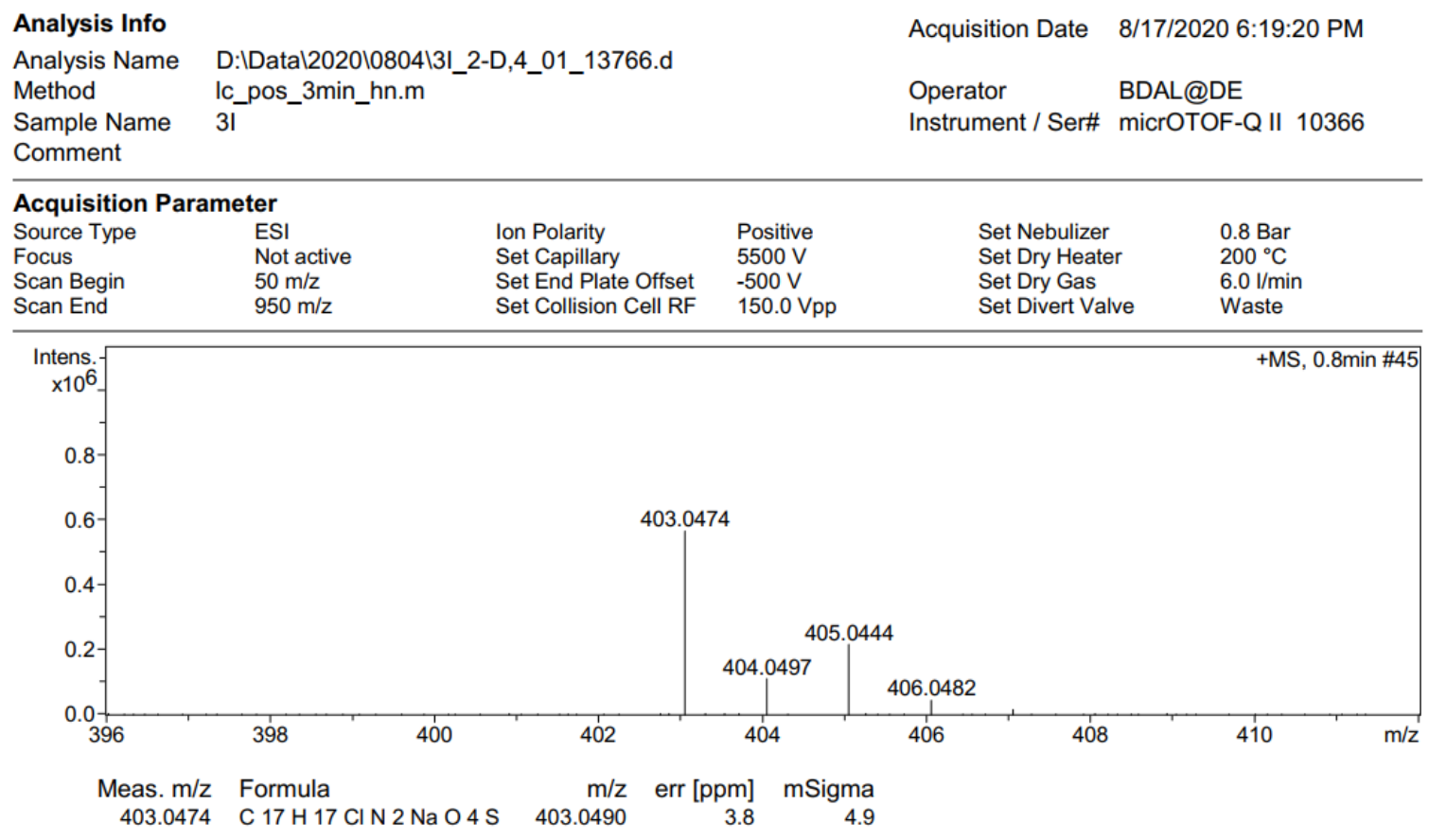

\section{HRMS Spectrum of Compound $5 i$}

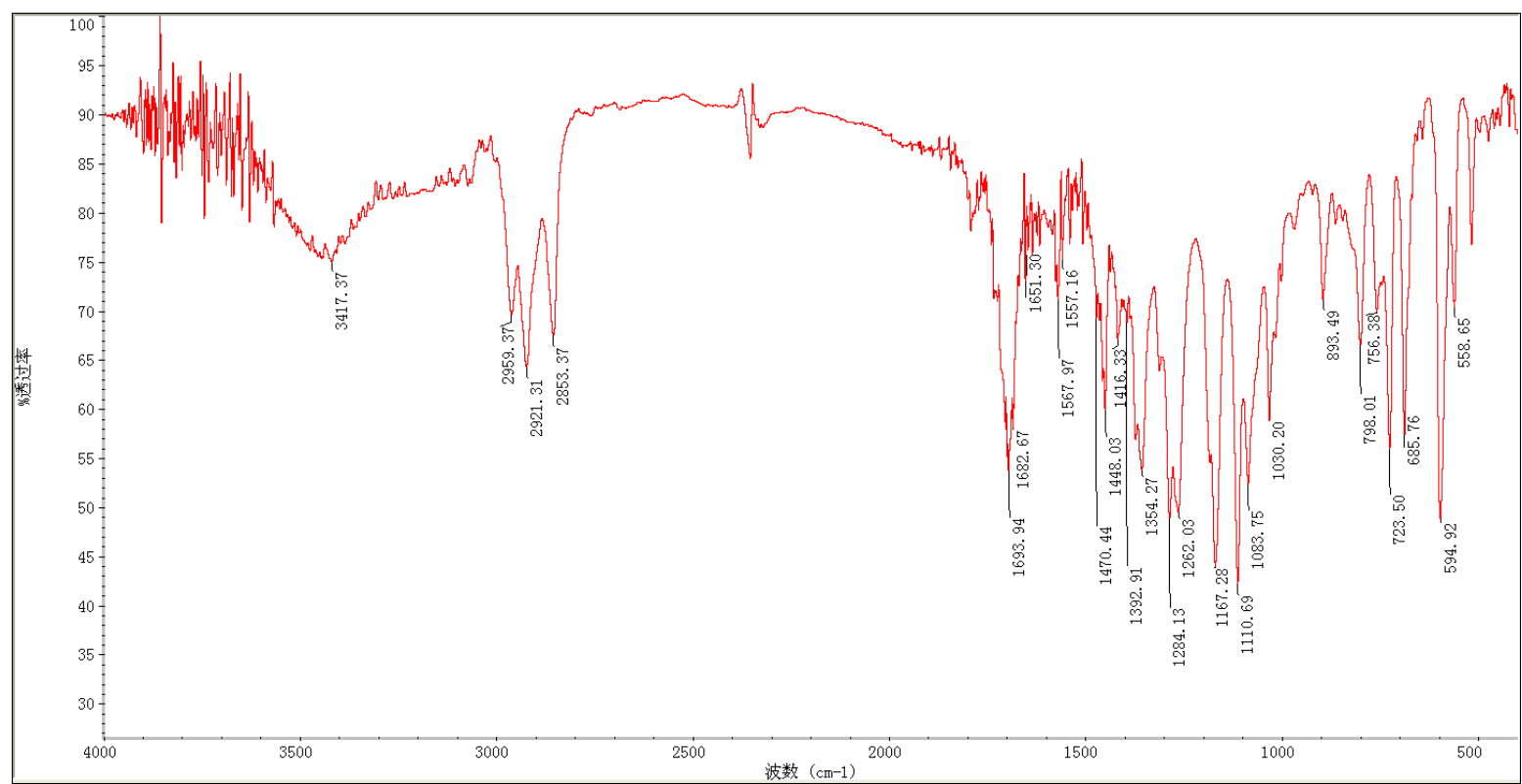

IR Spectrum of Compound 5i 
<smiles>O=C(c1cccc(Br)c1)N(N1CCOCC1)S(=O)(=O)c1ccccc1</smiles>

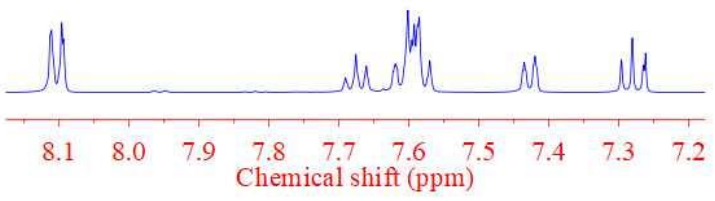

${ }^{1} \mathrm{H}$ NMR $\left(\mathrm{CDCl}_{3}, 500 \mathrm{MHz}\right)$

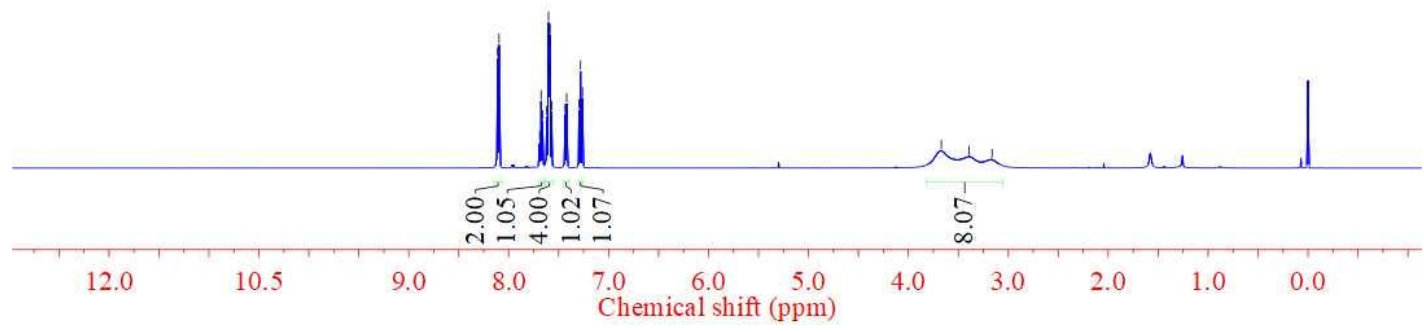

${ }^{1} \mathrm{H}$ NMR Spectrum of Compound $\mathbf{5 j}$

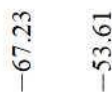
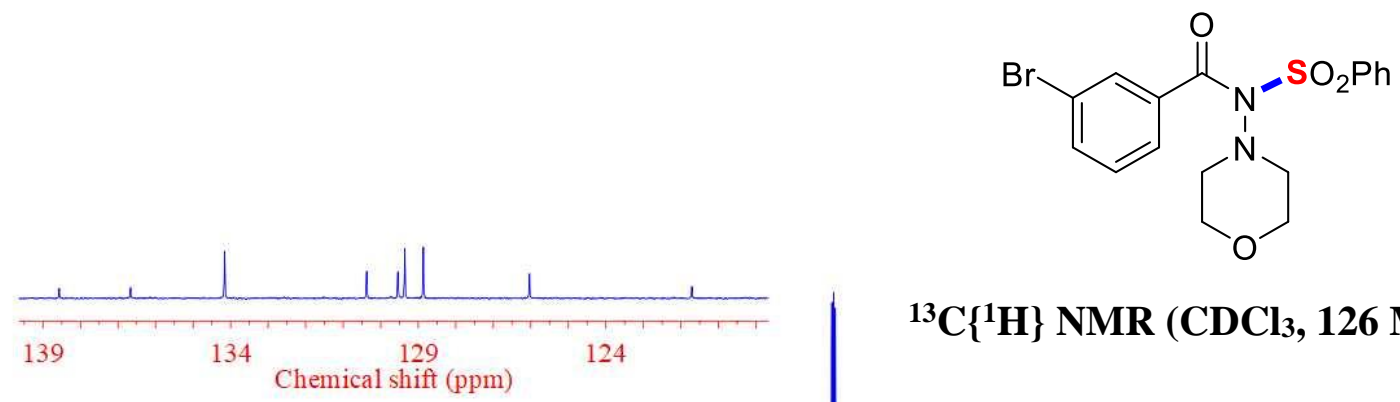

${ }^{13} \mathrm{C}\left\{{ }^{1} \mathrm{H}\right\} \mathrm{NMR}\left(\mathrm{CDCl}_{3}, 126 \mathrm{MHz}\right)$

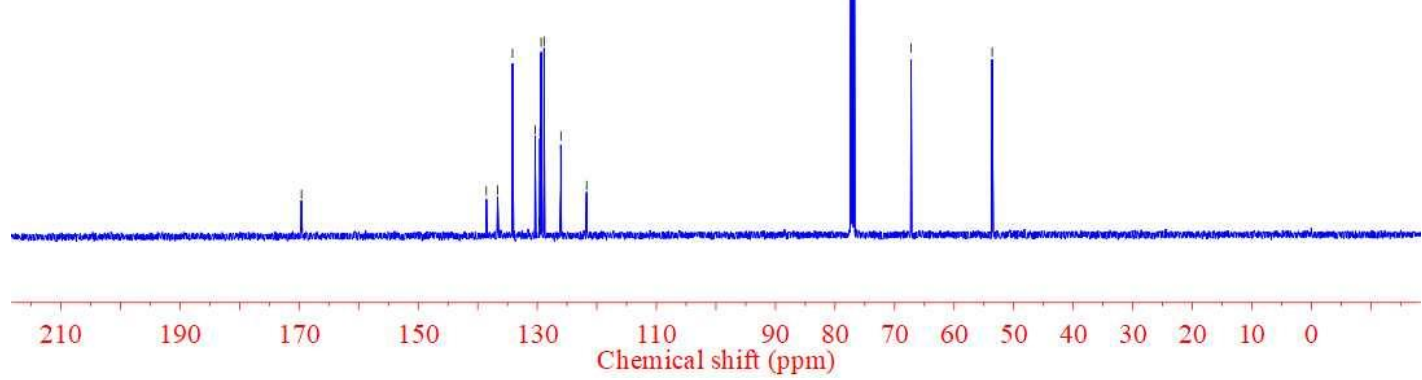

${ }^{13} \mathrm{C}\left\{{ }^{1} \mathrm{H}\right\}$ NMR Spectrum of Compound $5 \mathrm{j}$ 


\section{Mass Spectrum SmartFormula Report}

Analysis Info

Analysis Name

Method

(2020\0804|3J_2-D,5_01_13767.d

Ic_pos_3min_hn.m

$3 \mathrm{~J}$
Acquisition Date

8/17/2020 6:23:36 PM

Operator BDAL@DE

Instrument / Ser\# micrOTOF-Q II 10366

Comment

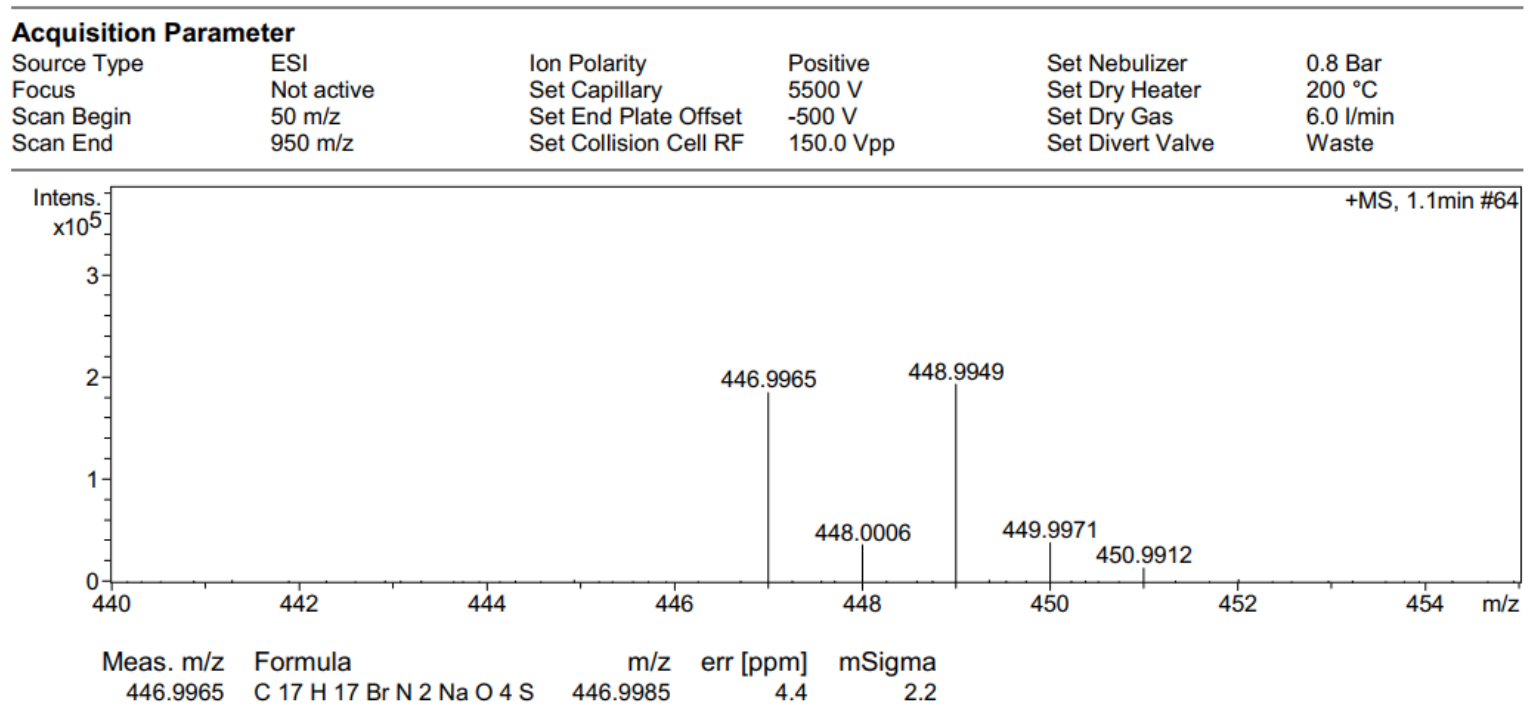

HRMS Spectrum of Compound 5j

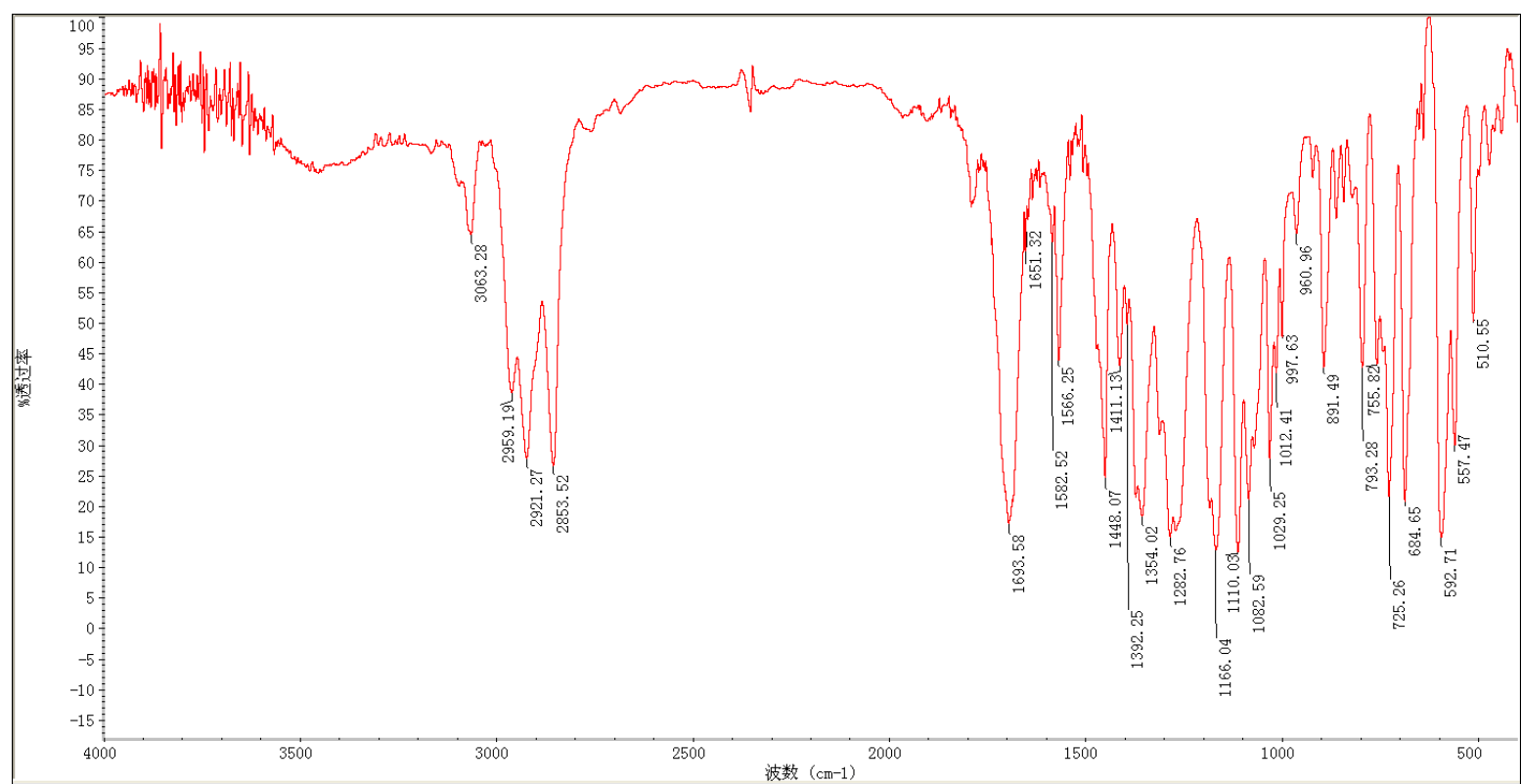

IR Spectrum of Compound $\mathbf{5 j}$ 
<smiles>N#Cc1cccc(C(=O)N(N2CCOCC2)S(=O)(=O)c2ccccc2)c1</smiles>

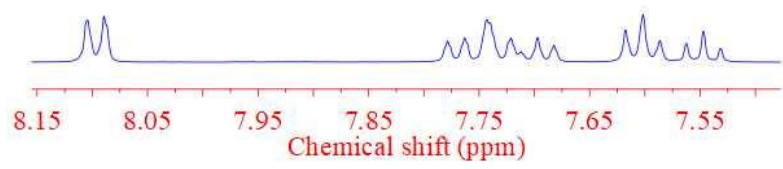

${ }^{1} \mathrm{H}$ NMR (CDCl3, $\left.500 \mathrm{MHz}\right)$

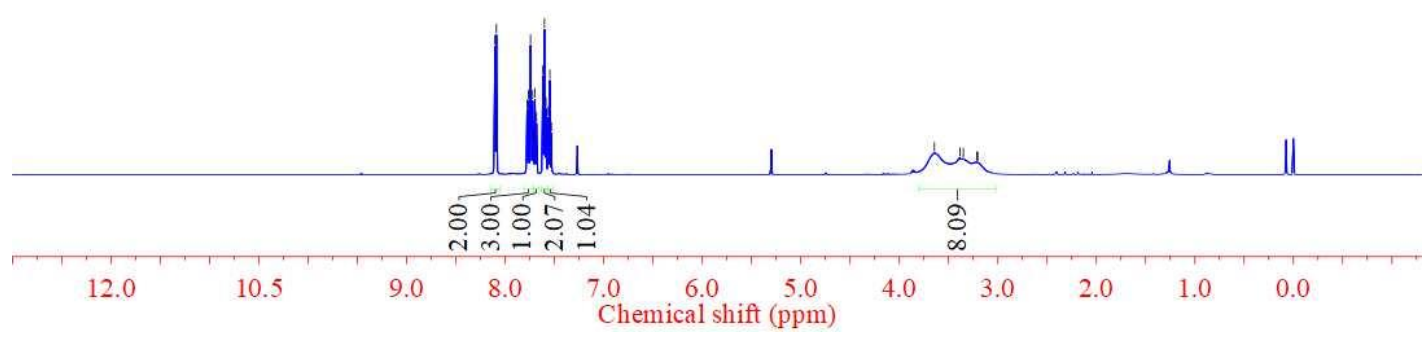

${ }^{1} \mathrm{H}$ NMR Spectrum of Compound 5k

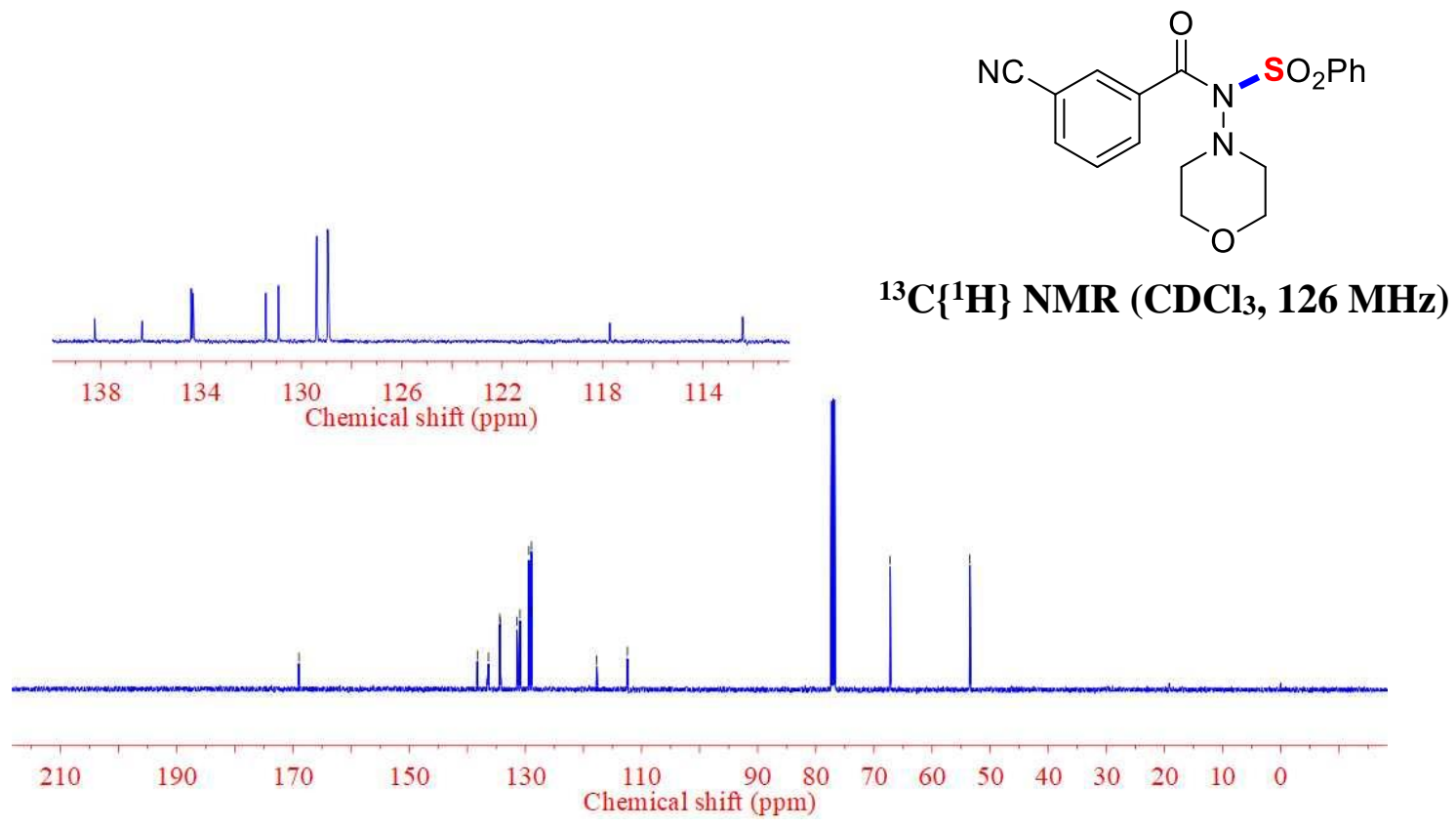

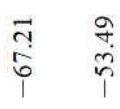

${ }^{13} \mathrm{C}\left\{{ }^{1} \mathrm{H}\right\}$ NMR Spectrum of Compound $5 \mathrm{k}$ 


\section{Mass Spectrum SmartFormula Report}

Analysis Info

Analysis Name

Method

Sample Name

Comment

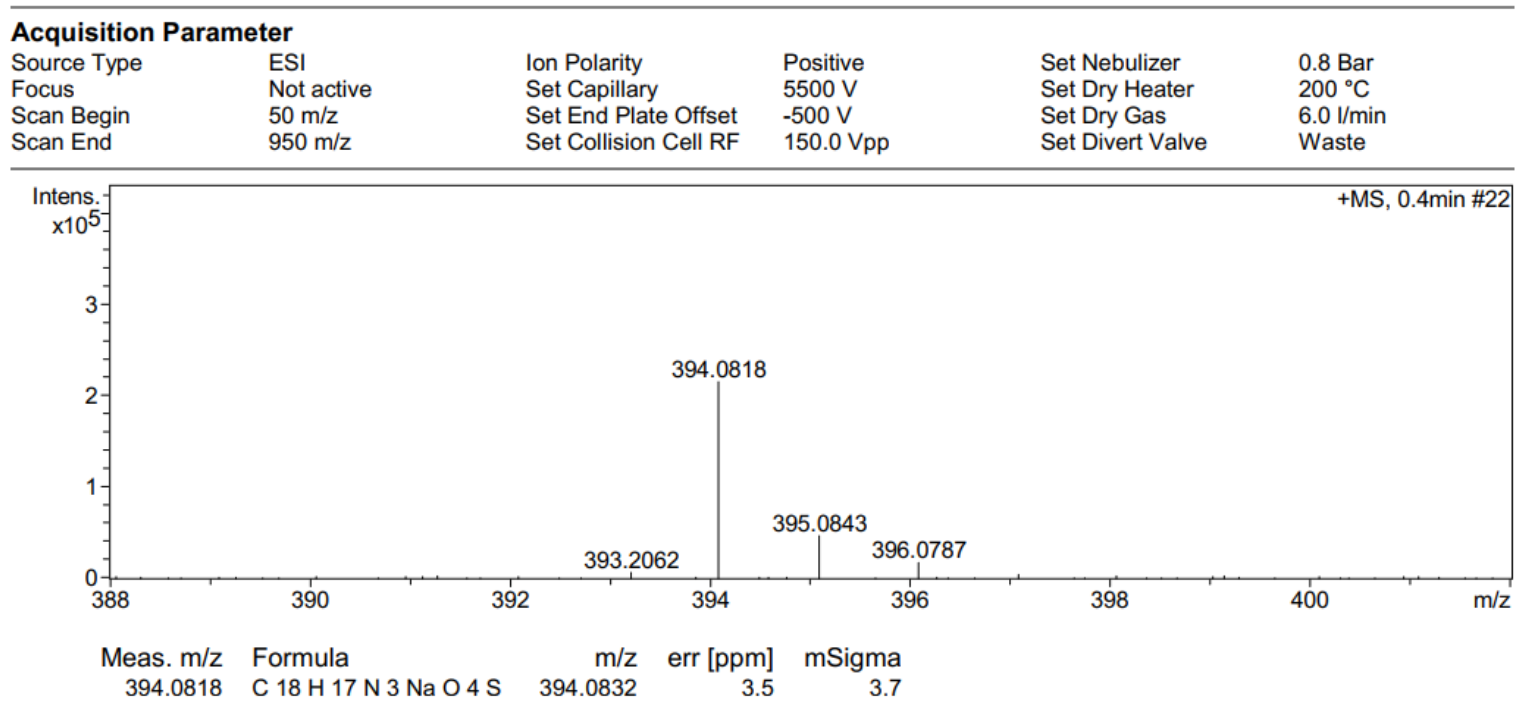

HRMS Spectrum of Compound 5k

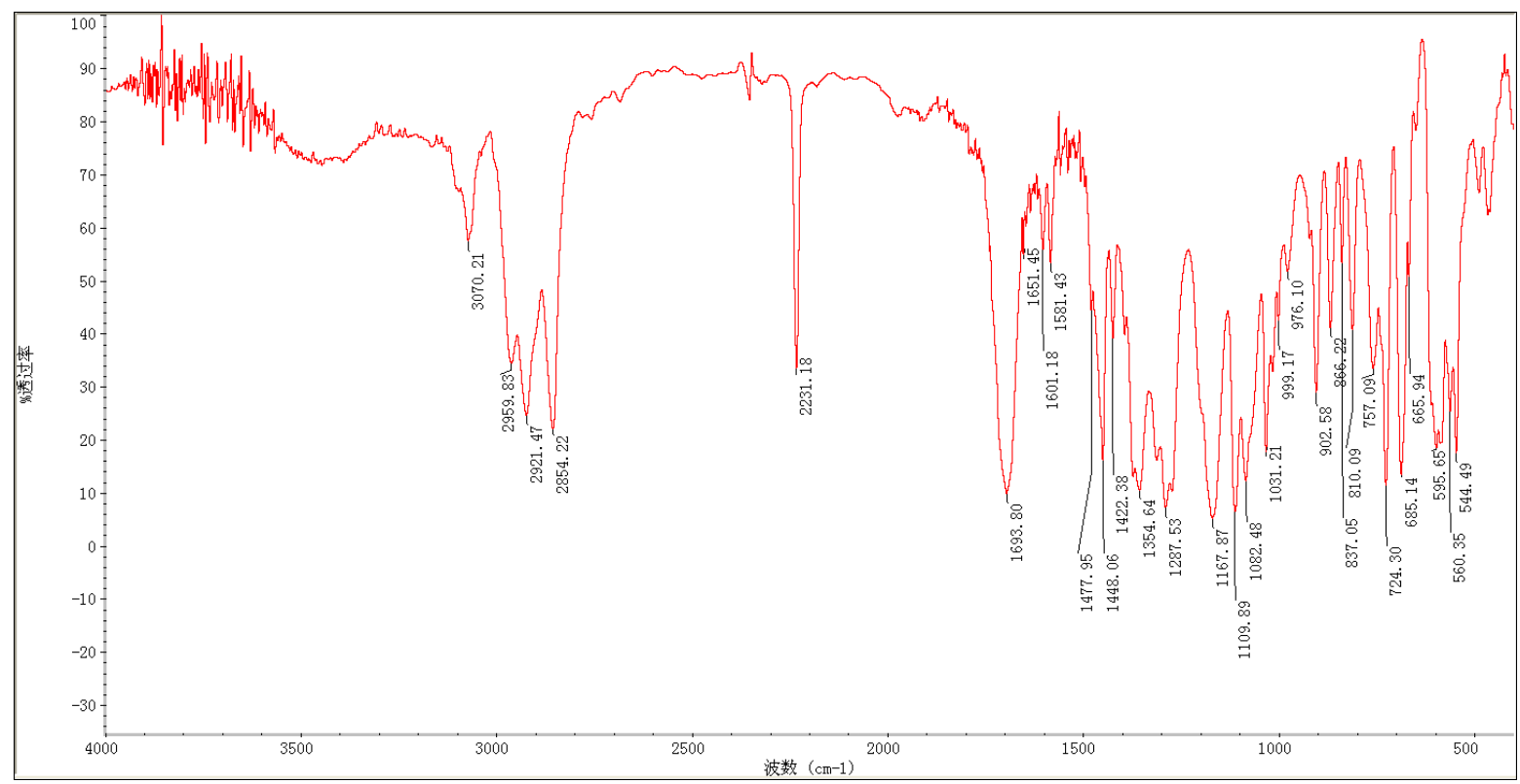

IR Spectrum of Compound 5k 
<smiles>O=C(c1cccc([N+](=O)[O-])c1)N(N1CCOCC1)S(=O)(=O)c1ccccc1</smiles>

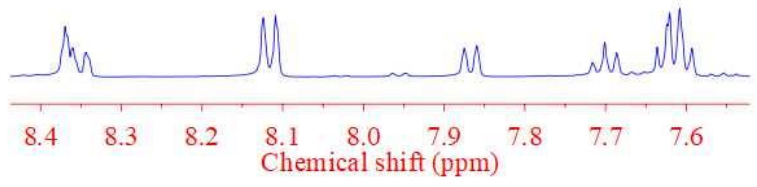

${ }^{1} \mathrm{H}$ NMR $\left(\mathrm{CDCl}_{3}, 500 \mathrm{MHz}\right)$

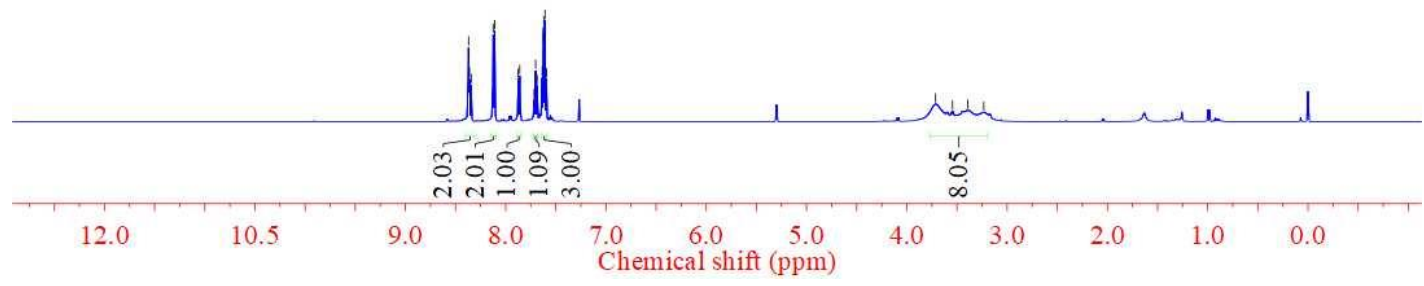

${ }^{1}$ H NMR Spectrum of Compound 51

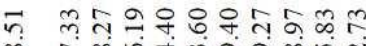

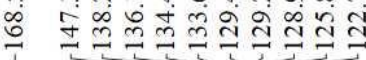

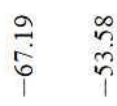

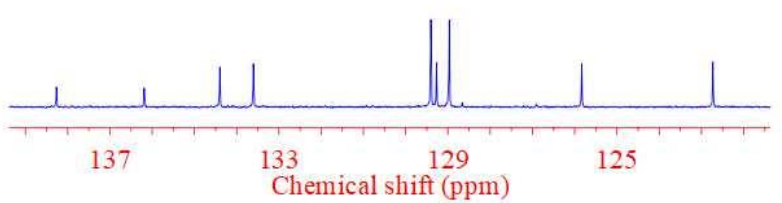<smiles>O=C(c1cccc([N+](=O)[O-])c1)N(N1CCOCC1)S(=O)(=O)c1ccccc1</smiles>

${ }^{13} \mathrm{C}\left\{{ }^{1} \mathrm{H}\right\} \mathrm{NMR}(\mathrm{CDCl} 3,126 \mathrm{MHz})$

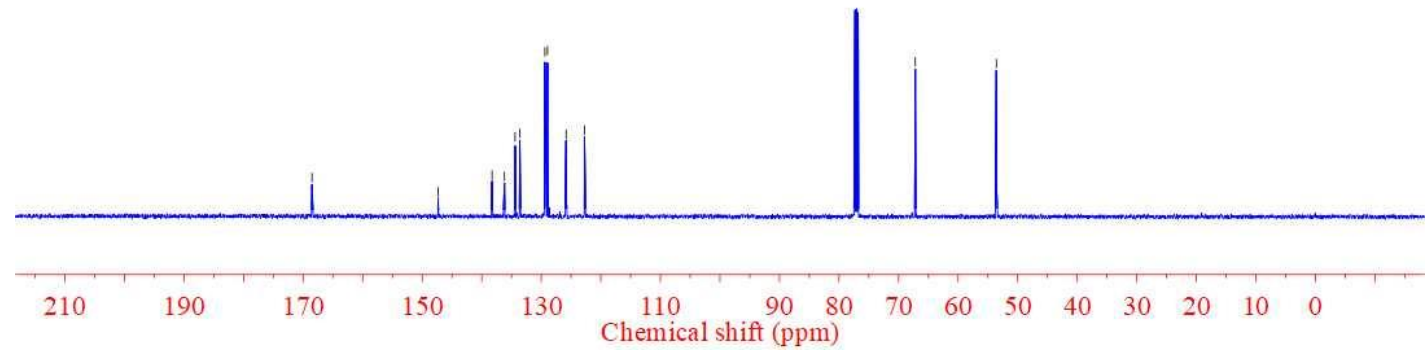

${ }^{13} \mathrm{C}\left\{{ }^{1} \mathrm{H}\right\}$ NMR Spectrum of Compound $5 \mathrm{I}$ 


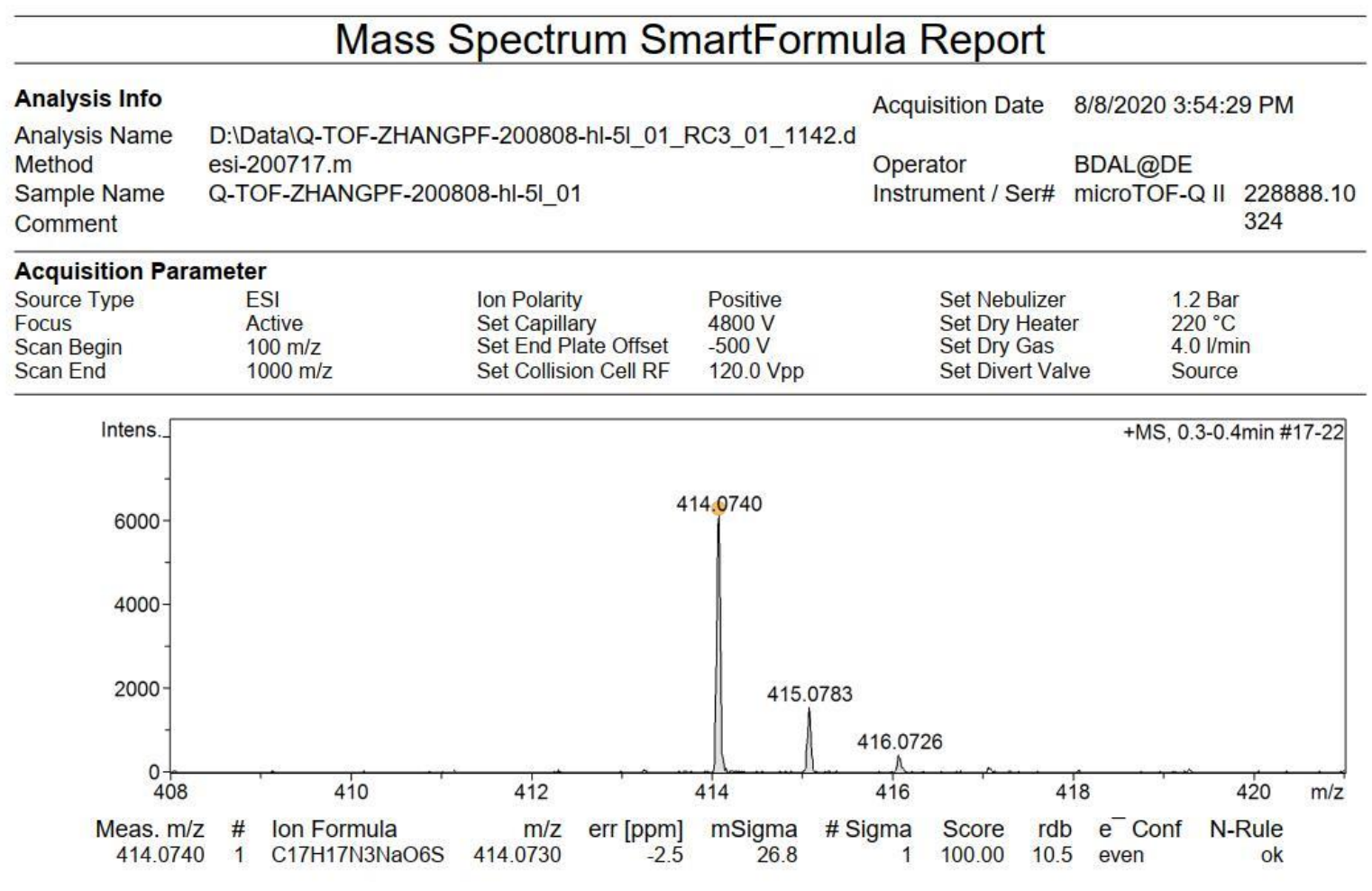

\section{HRMS Spectrum of Compound 51}

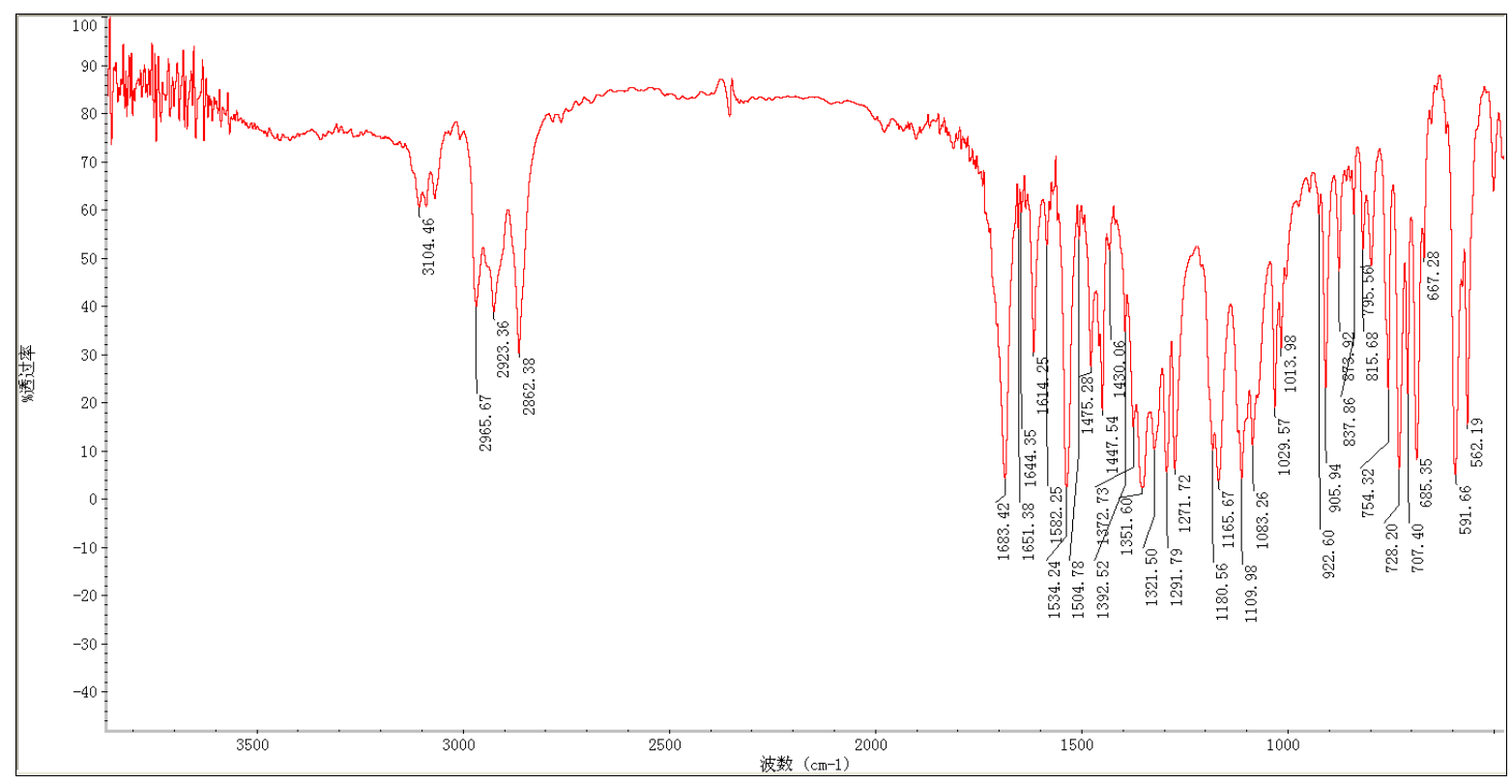

IR Spectrum of Compound 51 
<smiles>O=C(c1ccccc1F)N(N1CCOCC1)S(=O)(=O)c1ccccc1</smiles>

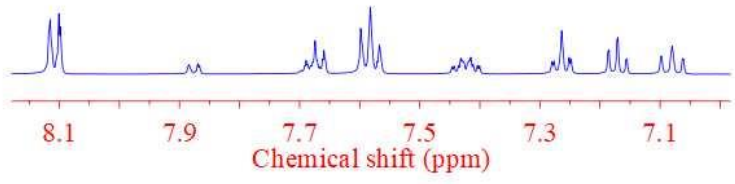

${ }^{1} \mathrm{H}$ NMR $\left(\mathrm{CDCl}_{3}, 500 \mathrm{MHz}\right)$

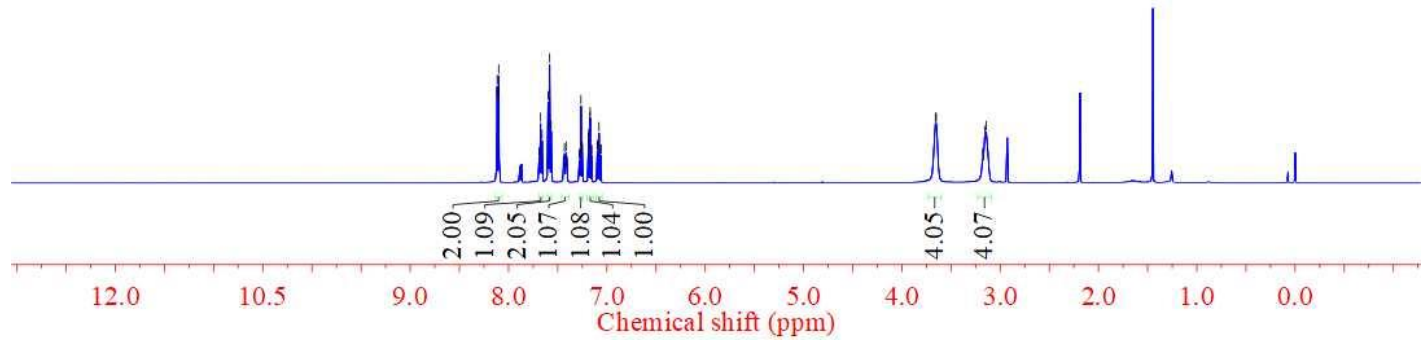

${ }^{1} \mathrm{H}$ NMR Spectrum of Compound $5 \mathrm{~m}$

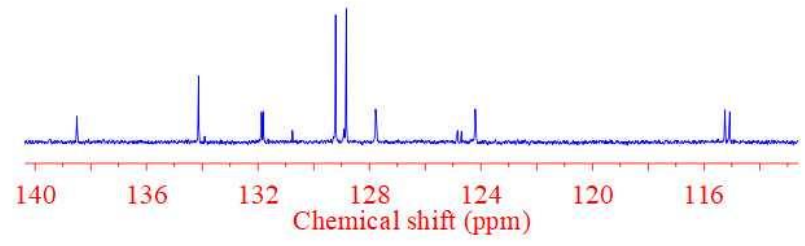

$\begin{array}{ll} \pm & \infty \\ i & n\end{array}$<smiles>O=C(c1ccccc1F)N(N1CCOCC1)S(=O)(=O)c1ccccc1</smiles>

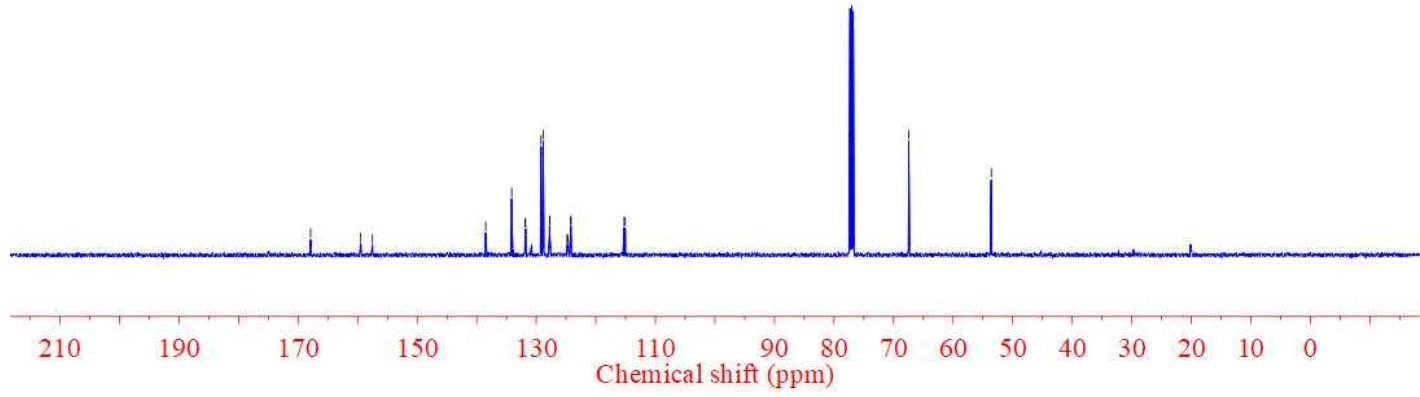

${ }^{13} \mathbf{C}\left\{{ }^{1} \mathbf{H}\right\}$ NMR Spectrum of Compound 5m 
<smiles>O=C(c1ccccc1F)N(N1CCOCC1)N1CCOCC1</smiles>

${ }^{19} \mathrm{~F}\left\{{ }^{1} \mathrm{H}\right\}$ NMR $\left(\mathrm{CDCl}_{3}, 471 \mathrm{MHz}\right)$

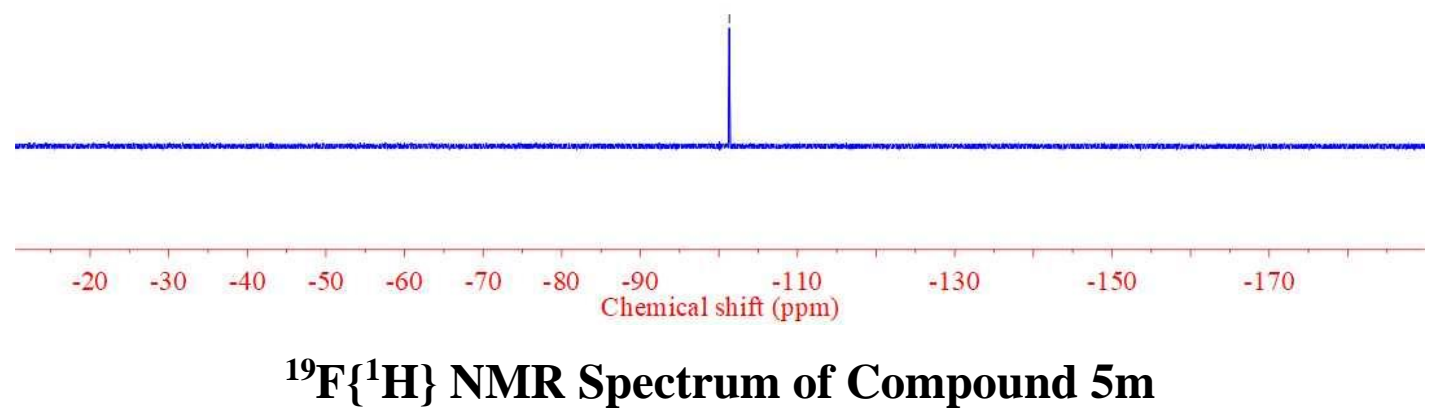

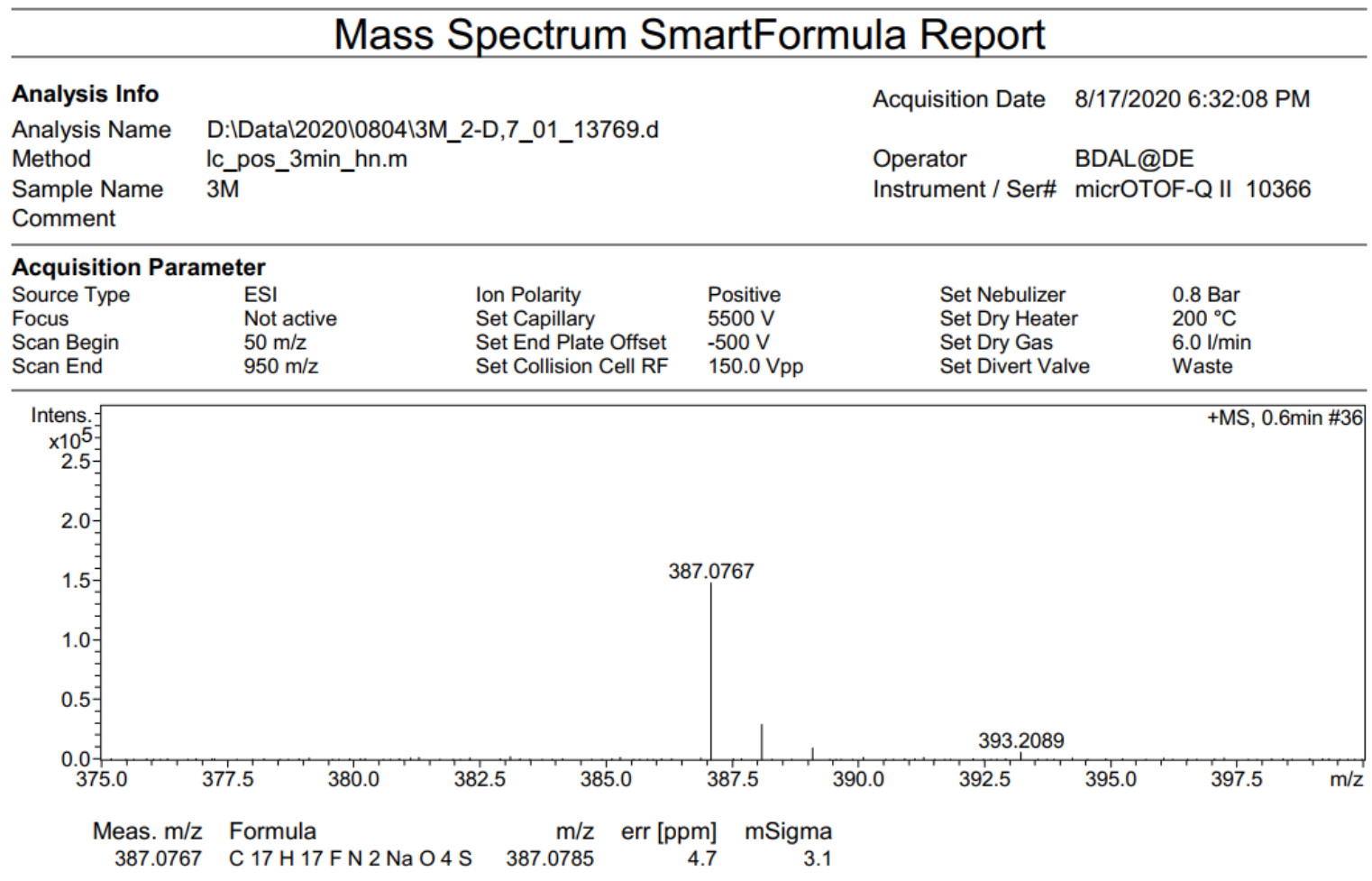

HRMS Spectrum of Compound $5 \mathrm{~m}$ 


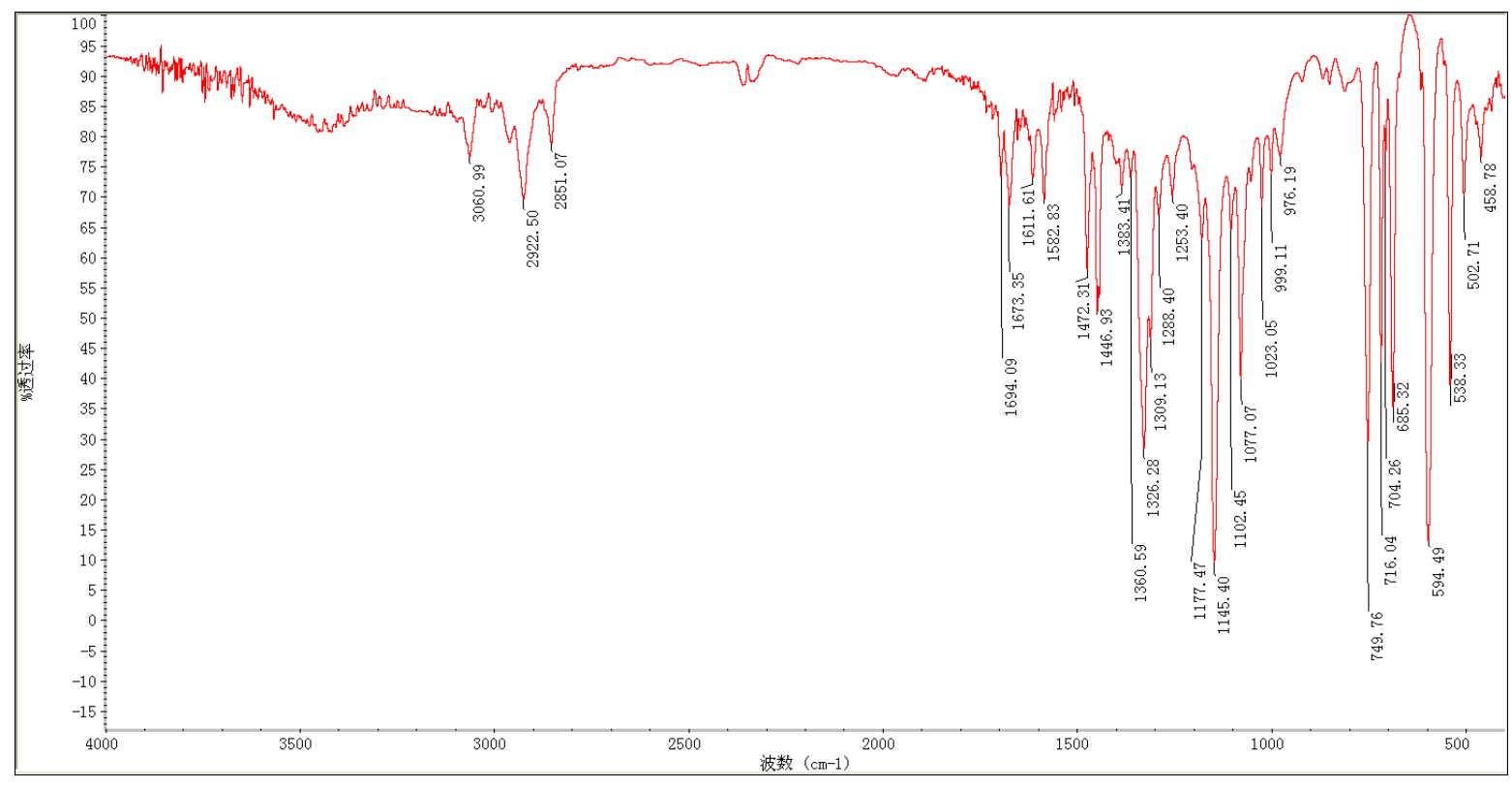

IR Spectrum of Compound 5m

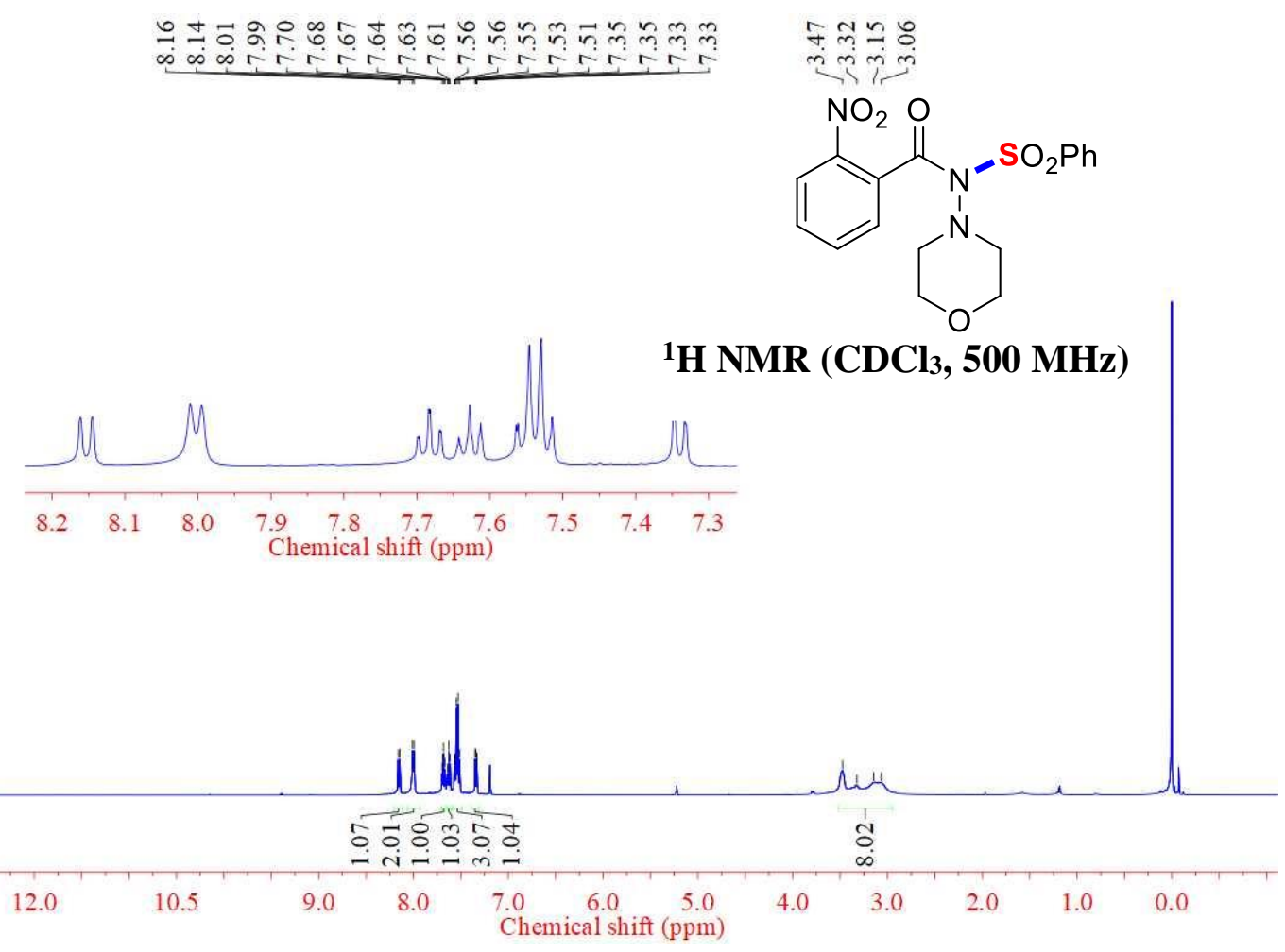

${ }^{1} \mathrm{H}$ NMR Spectrum of Compound $5 n$ 


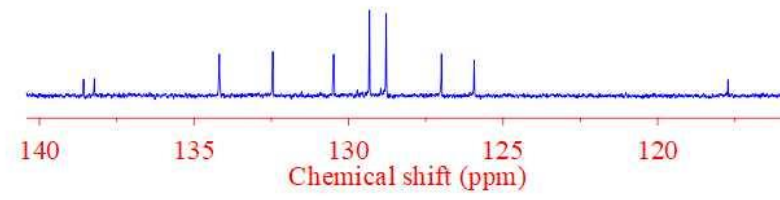<smiles>O=C(OS(=O)(=O)c1ccccc1)N(C(=O)c1ccccc1[N+](=O)[O-])N1CCOCC1</smiles>

${ }^{13} \mathrm{C}\left\{{ }^{1} \mathrm{H}\right\} \mathrm{NMR}\left(\mathrm{CDCl}_{3}, 126 \mathrm{MHz}\right)$

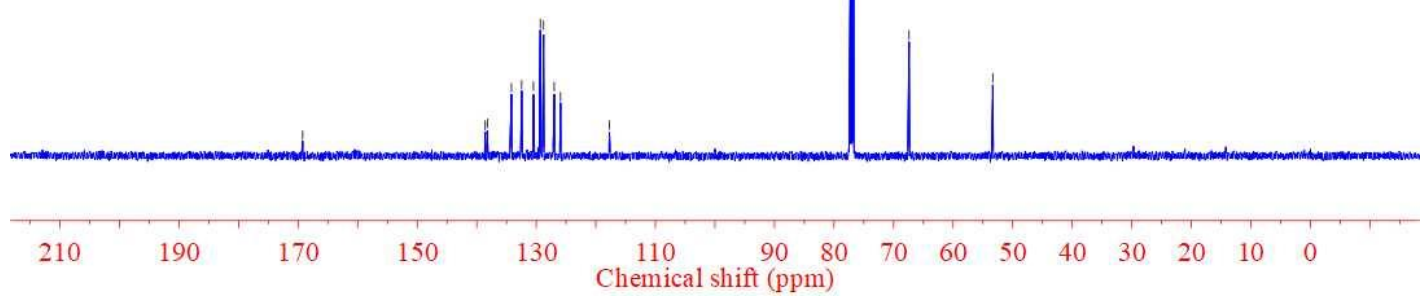

${ }^{13} \mathbf{C}\left\{{ }^{1} \mathrm{H}\right\}$ NMR Spectrum of Compound 5n

\section{Mass Spectrum SmartFormula Report}

Analysis Info

Analysis Name

Method

Sample Name

Comment

\section{Acquisition Parameter}

Source Type

Focus

Scan Begin

Scan End

$950 \mathrm{~m} / \mathrm{z}$

D:IDatal202010804|3N_2-D,8_01_13770.d

lc_pos_3min_hn.m

$3 \bar{N}$
Acquisition Date

Operator

$8 / 17 / 2020$ 6:36:25 PM

BDAL@DE

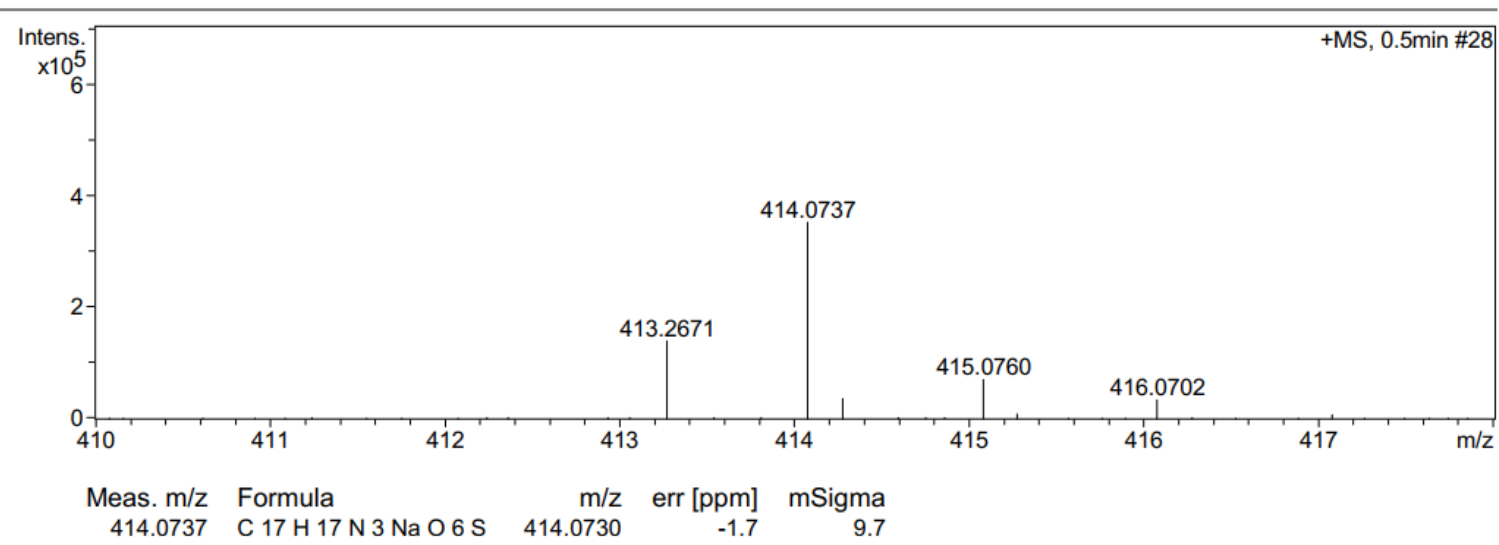

HRMS Spectrum of Compound 5n 


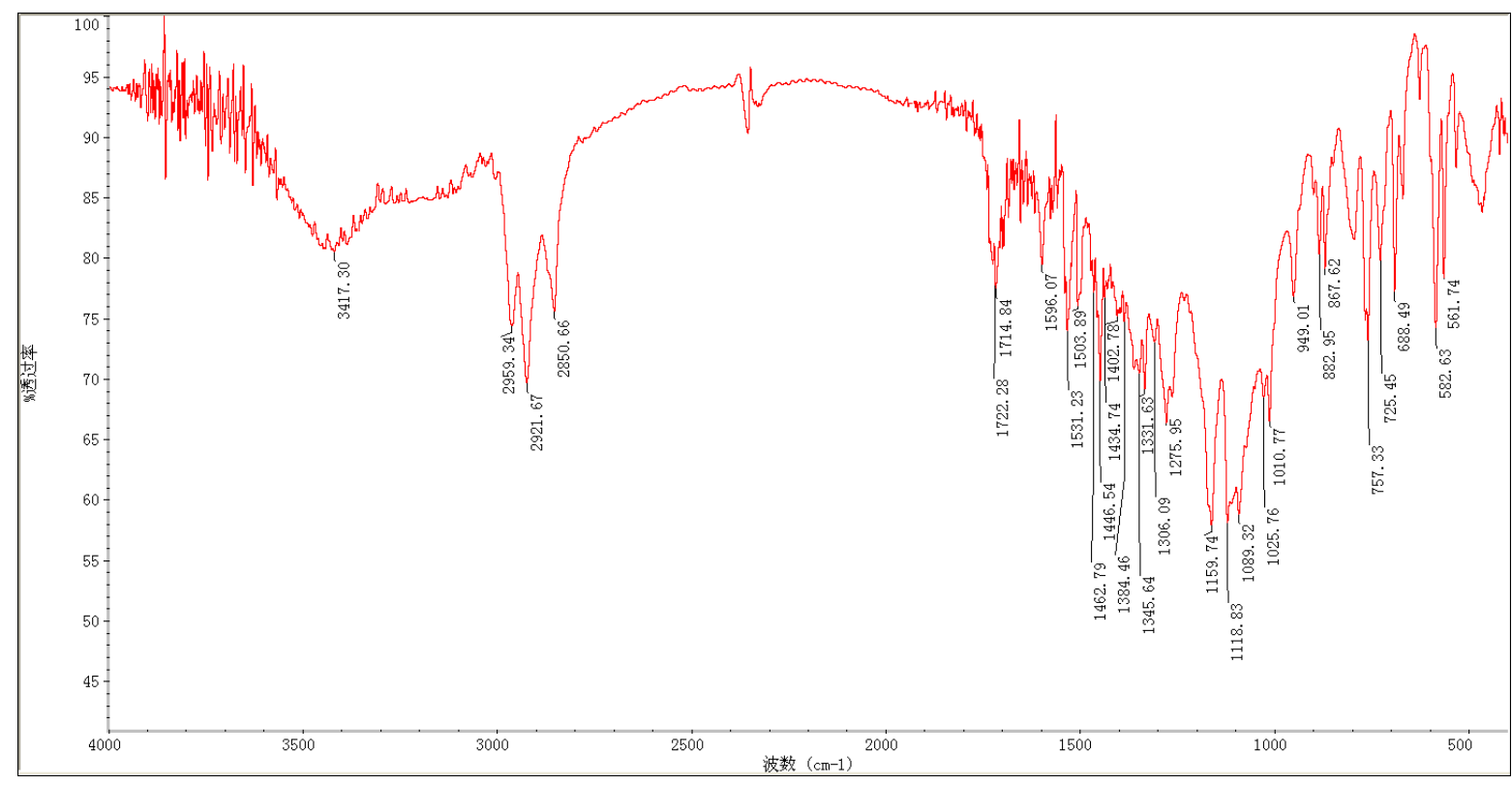

IR Spectrum of Compound 5n

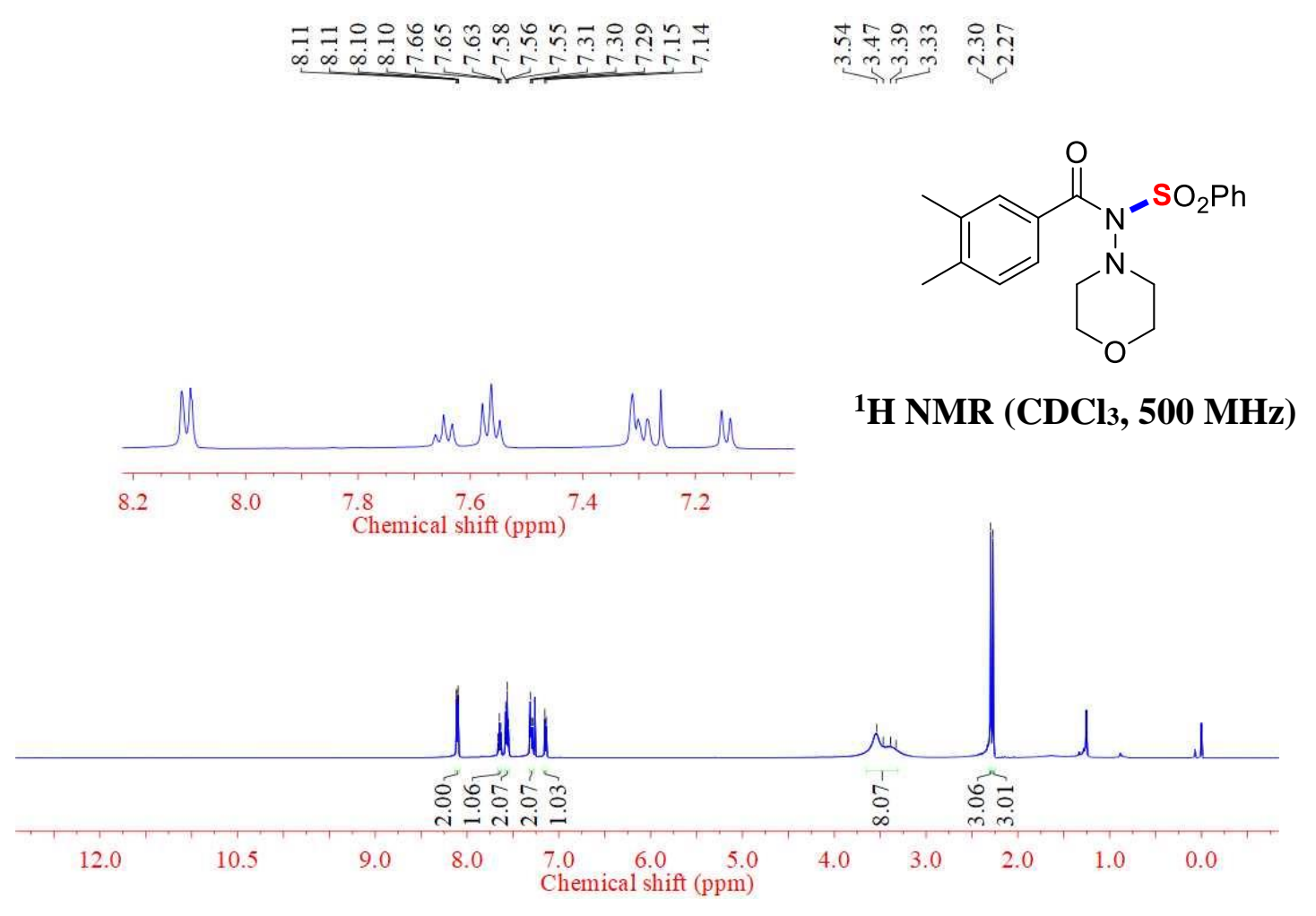

${ }^{1}$ H NMR Spectrum of Compound 50 


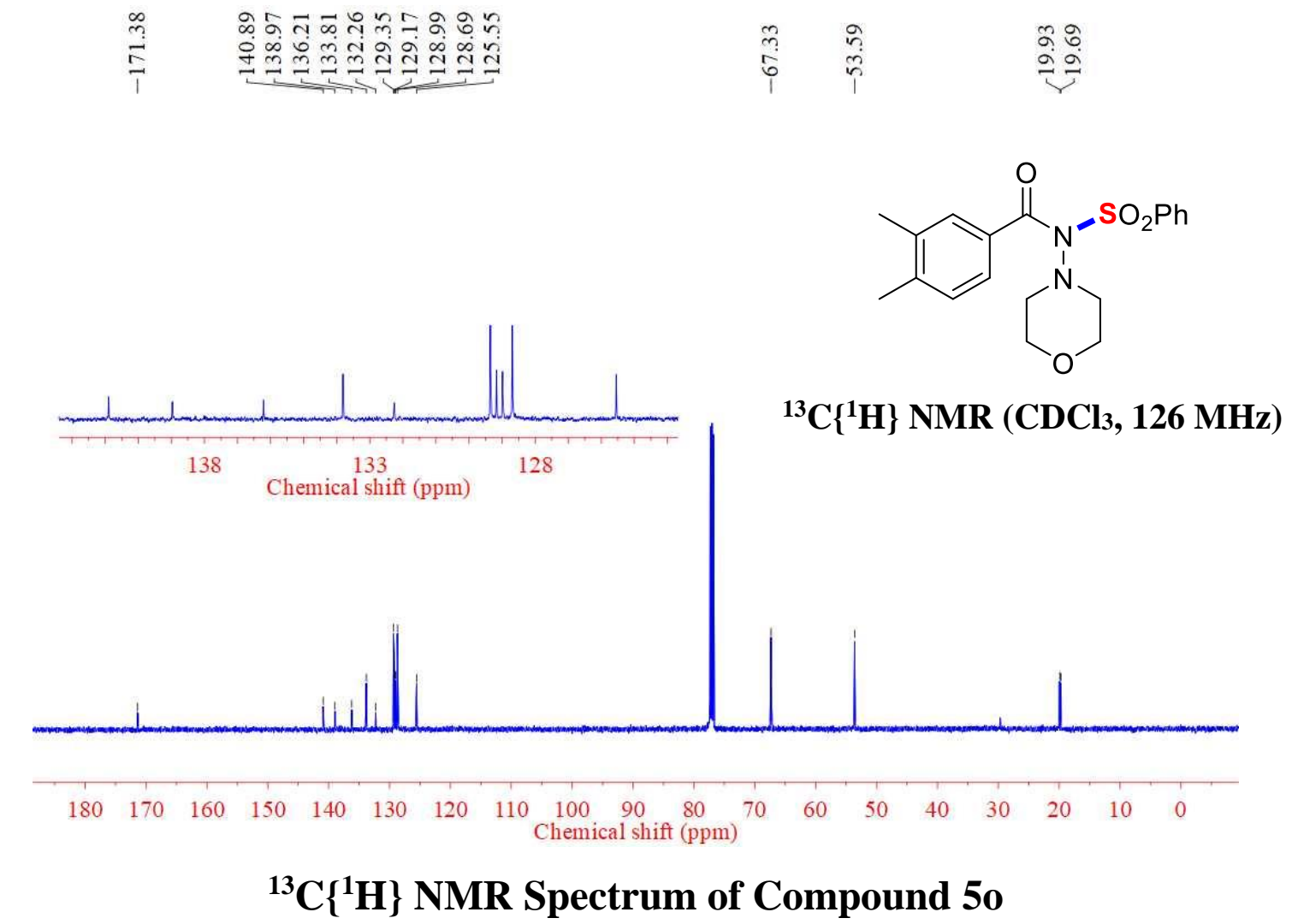

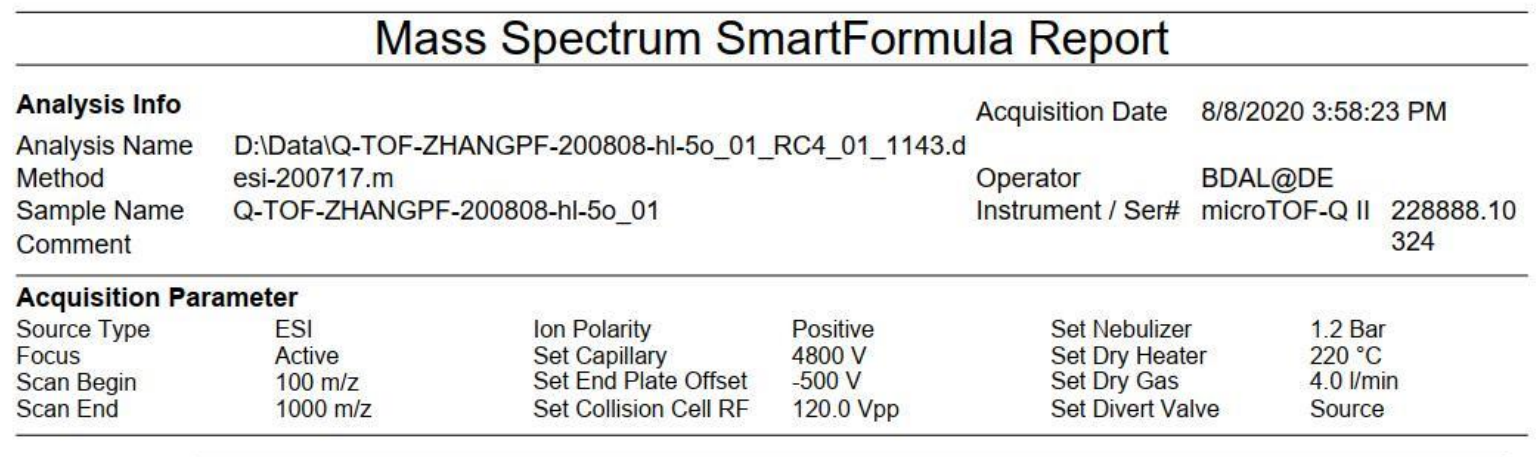

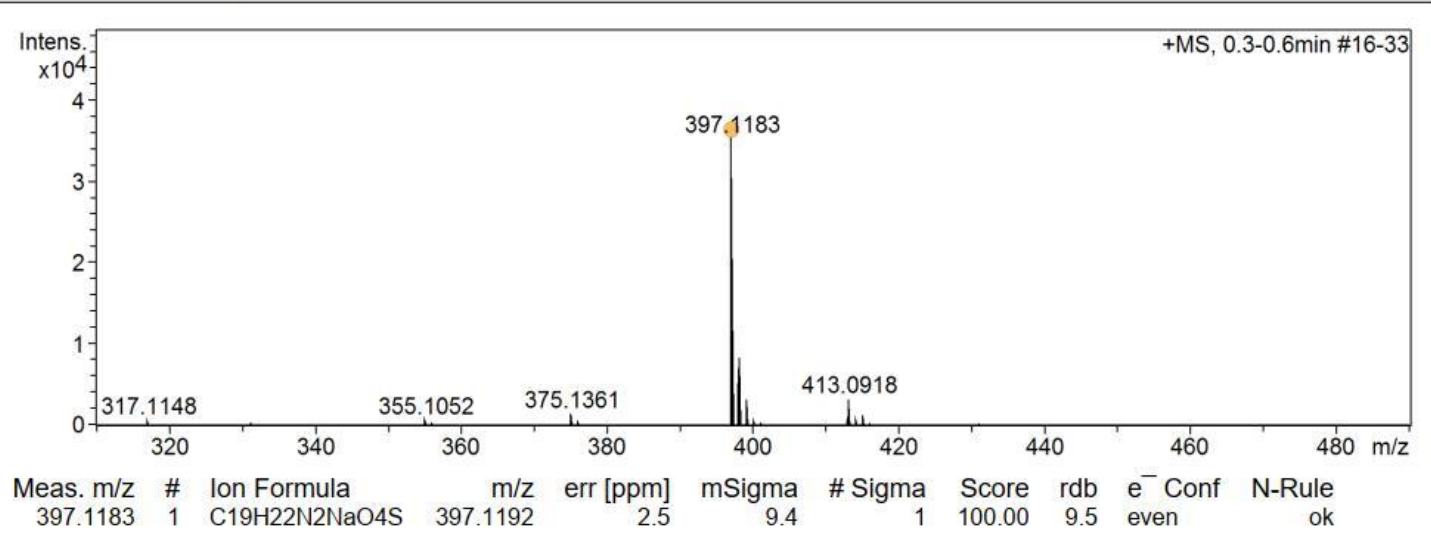

HRMS Spectrum of Compound 50 


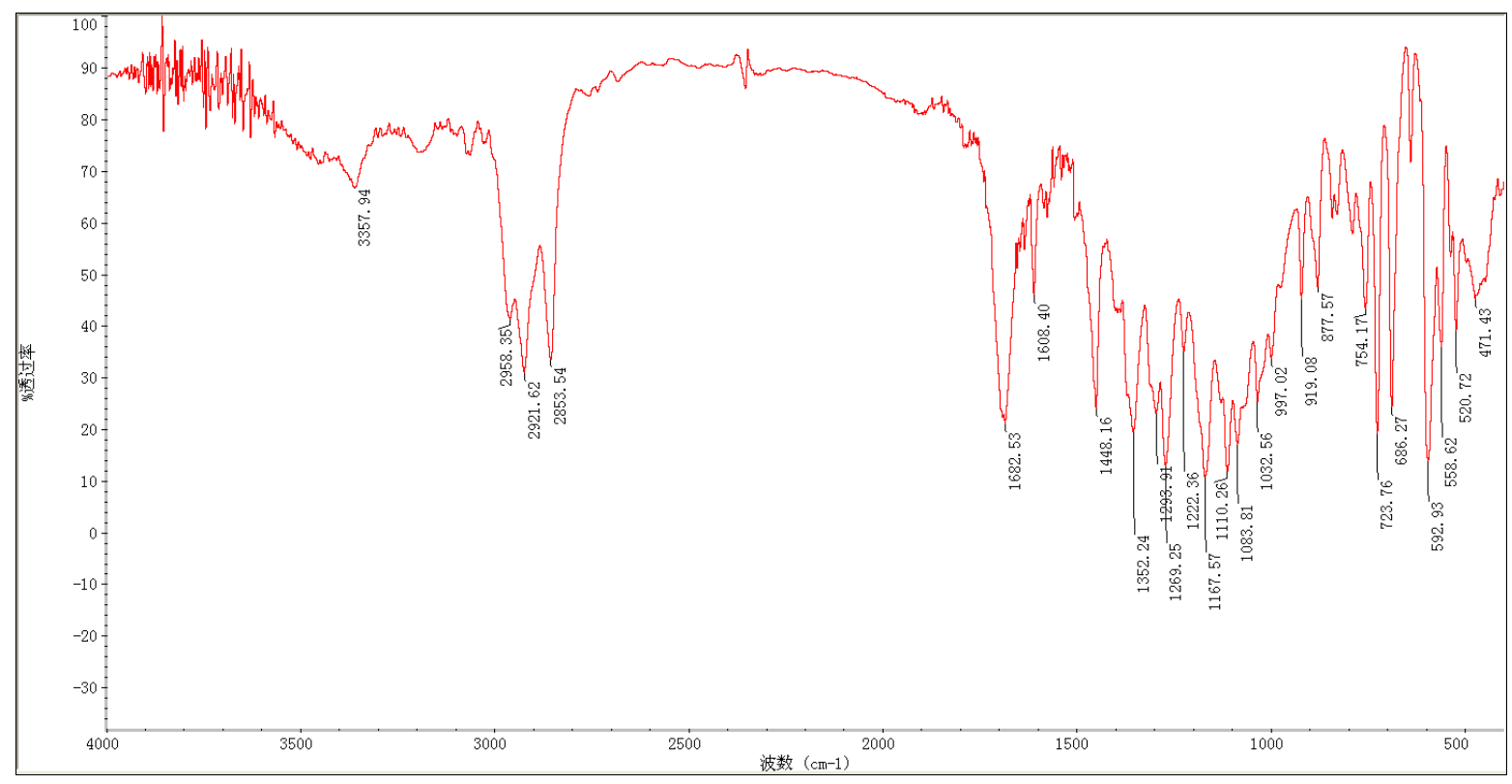

IR Spectrum of Compound 50

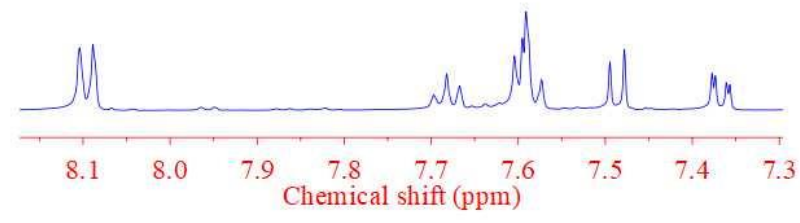<smiles>O=C(c1ccc(Cl)c(Cl)c1)N(N1CCOCC1)S(=O)(=O)c1ccccc1</smiles>

${ }^{1} \mathrm{H}$ NMR (CDCl3, $\left.500 \mathrm{MHz}\right)$

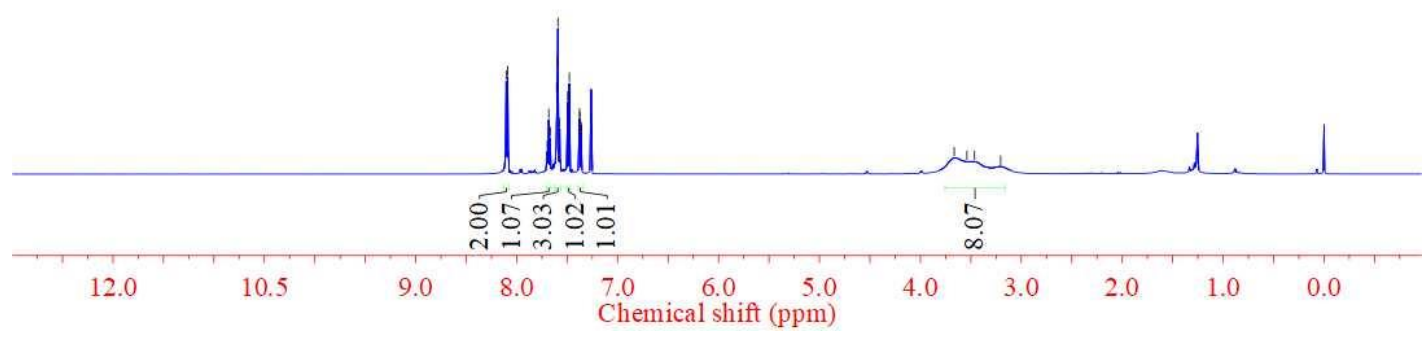

${ }^{1} \mathrm{H}$ NMR Spectrum of Compound 5p 


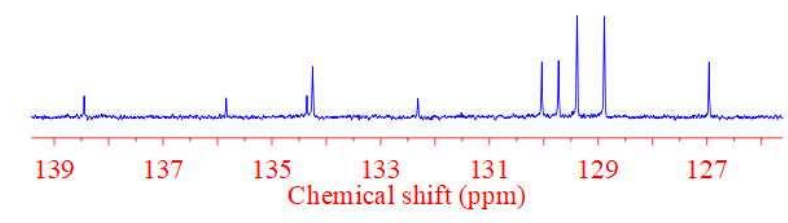<smiles>O=C(c1ccc(Cl)c(Cl)c1)N(N1CCOCC1)S(=O)(=O)c1ccccc1</smiles>

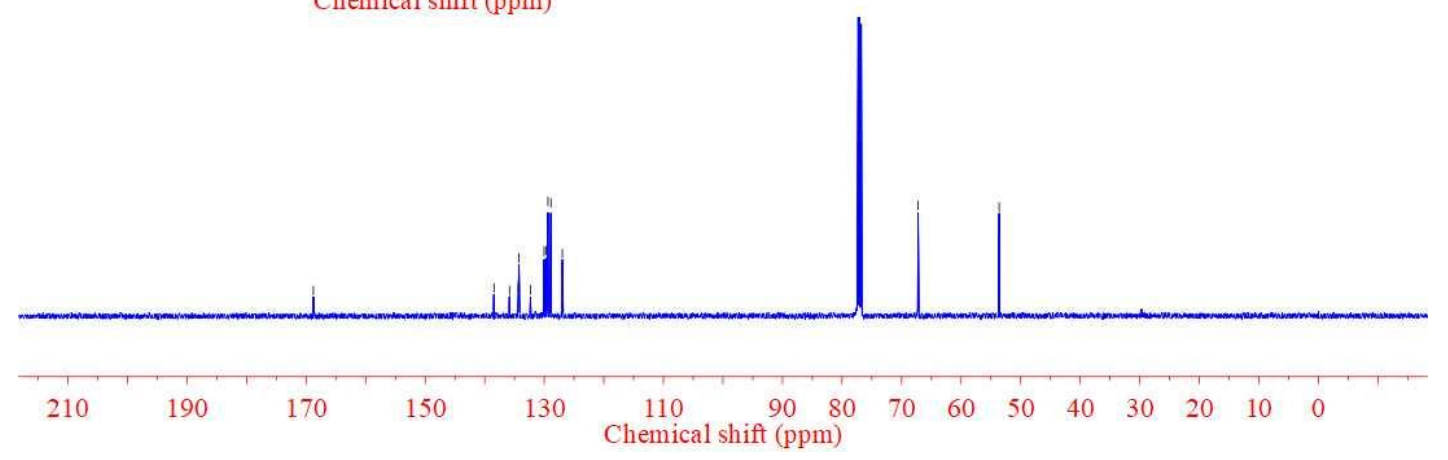

\section{${ }^{13} \mathrm{C}\left\{{ }^{1} \mathrm{H}\right\}$ NMR Spectrum of Compound $5 p$}

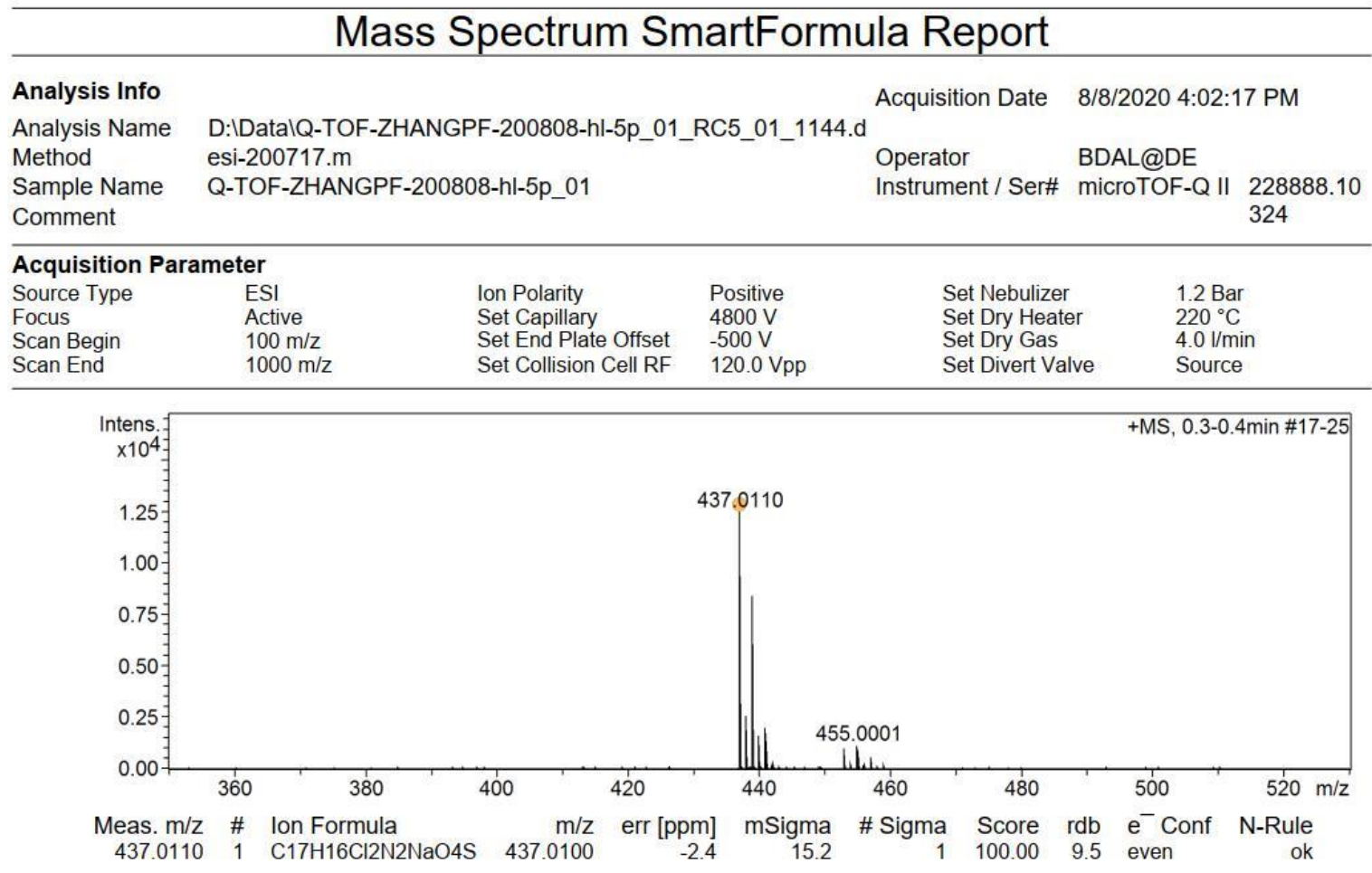

\section{HRMS Spectrum of Compound 5p}




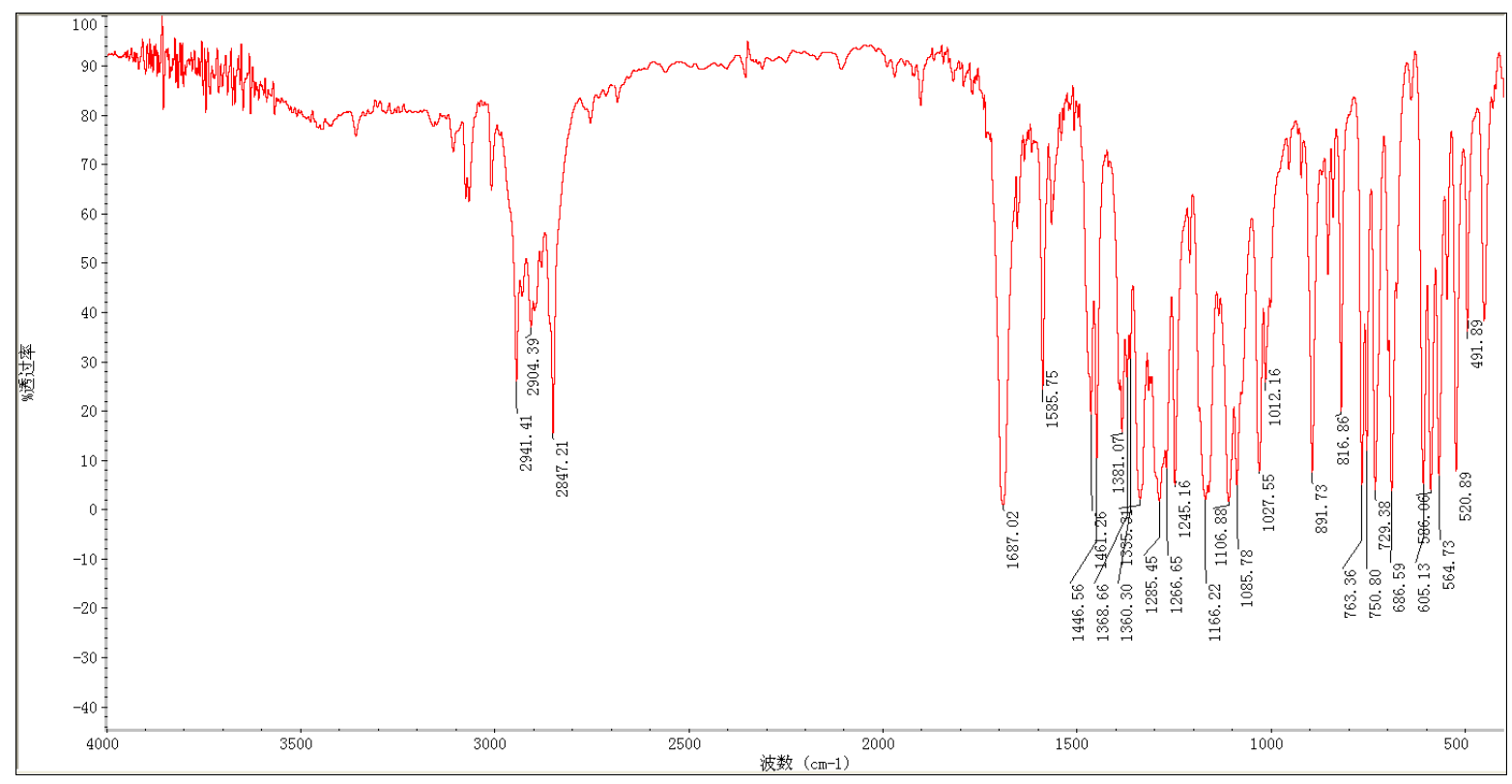

IR Spectrum of Compound 5p

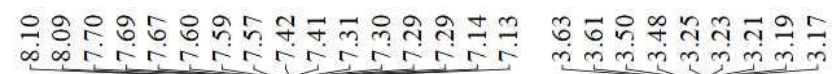

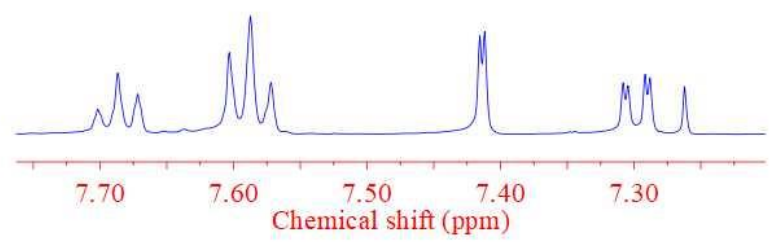<smiles>O=C(c1ccc(Cl)cc1Cl)N(N1CCOCC1)S(=O)(=O)c1ccccc1</smiles>

${ }^{1} \mathrm{H}$ NMR $\left(\mathrm{CDCl}_{3}, 500 \mathrm{MHz}\right)$

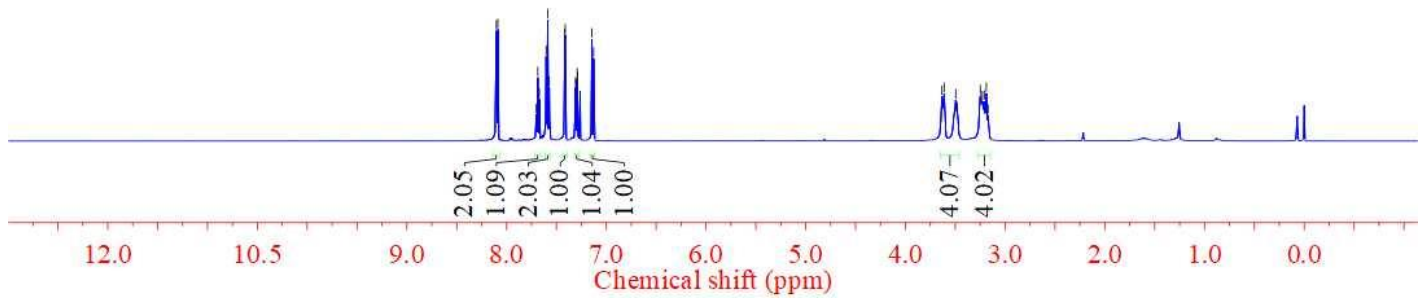

${ }^{1} \mathrm{H}$ NMR Spectrum of Compound 5q 


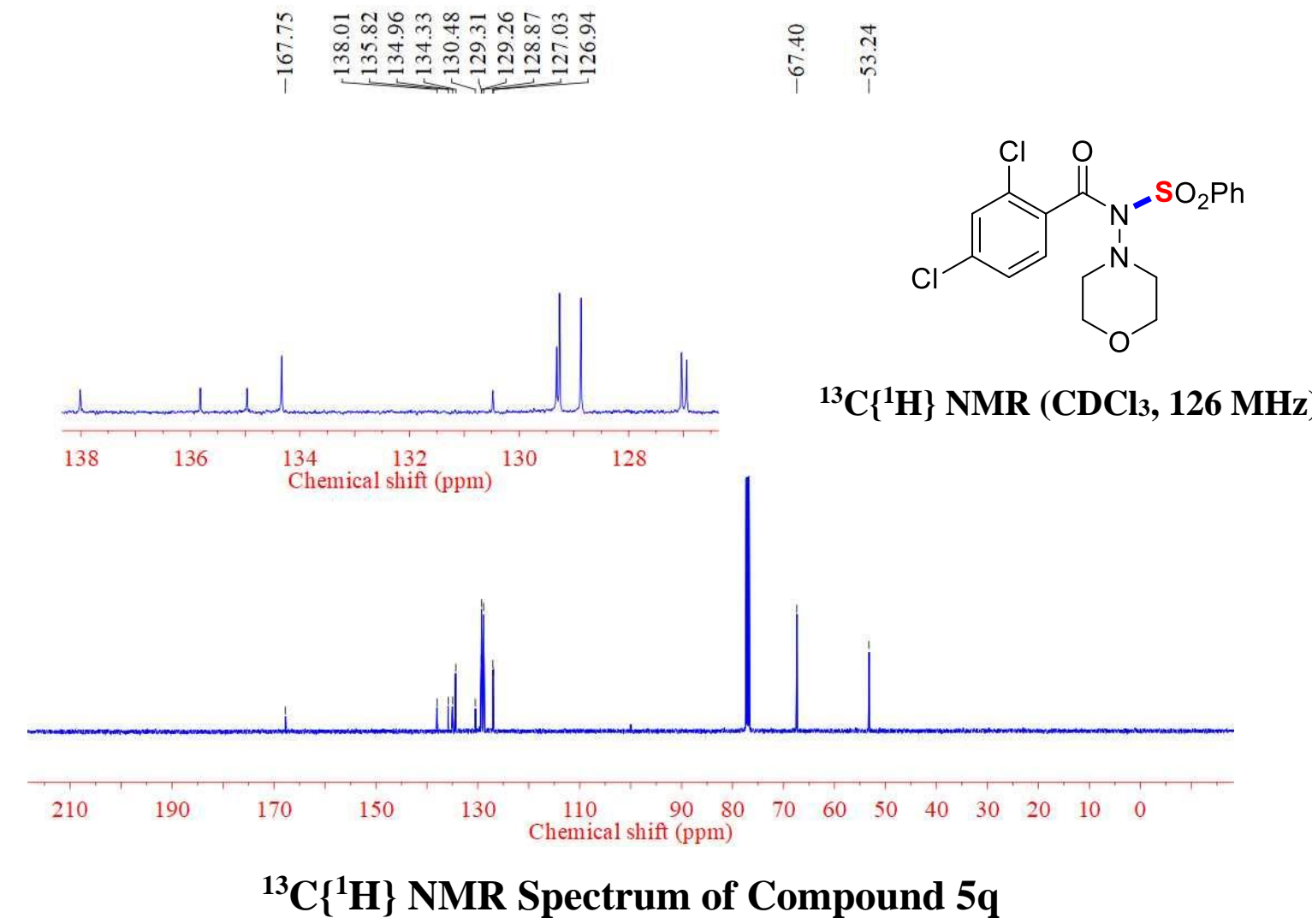

\section{Mass Spectrum SmartFormula Report}

Analysis Info

Analysis Name

Method

Sample Name

Comment

\section{Acquisition Parameter}

Source Type

Focus

Scan Begin

Scan End

D:IData|2020|0804|3Q_2-E,1_01_13771.d

lc_pos_3min_hn.m

$3 \bar{Q}$

Acquisition Date $\quad 8 / 17 / 2020$ 6:40:38 PM

Operator

BDAL@DE

Instrument / Ser\# micrOTOF-Q II 10366

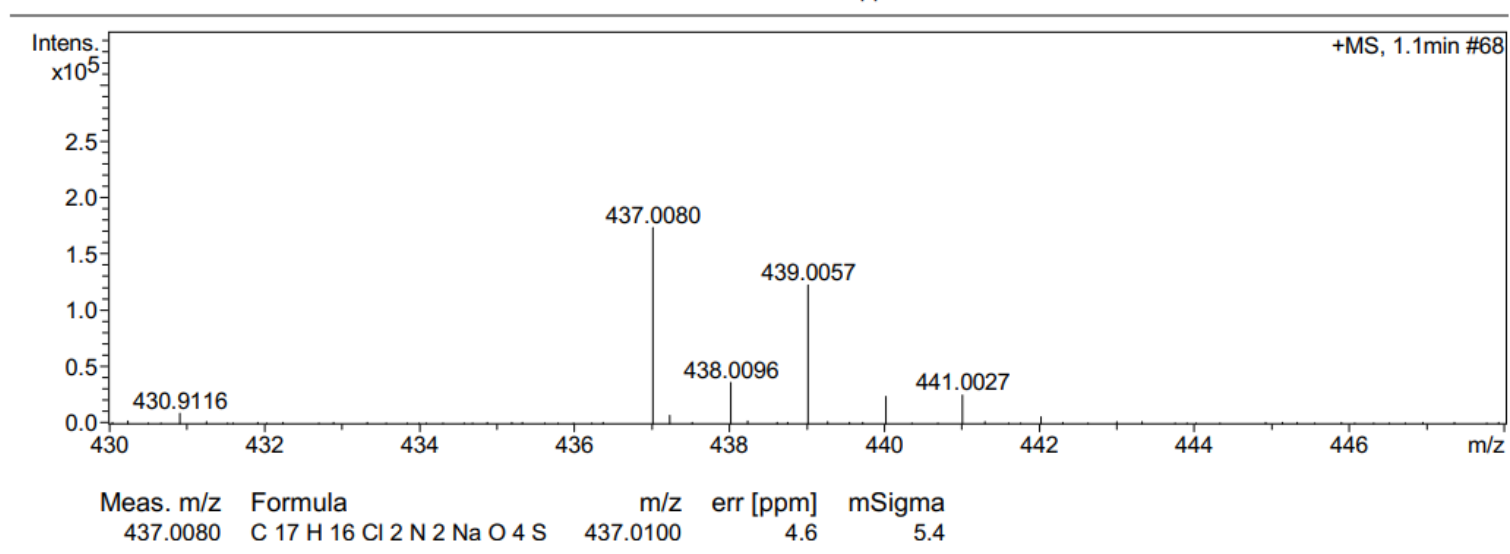

HRMS Spectrum of Compound 5q 


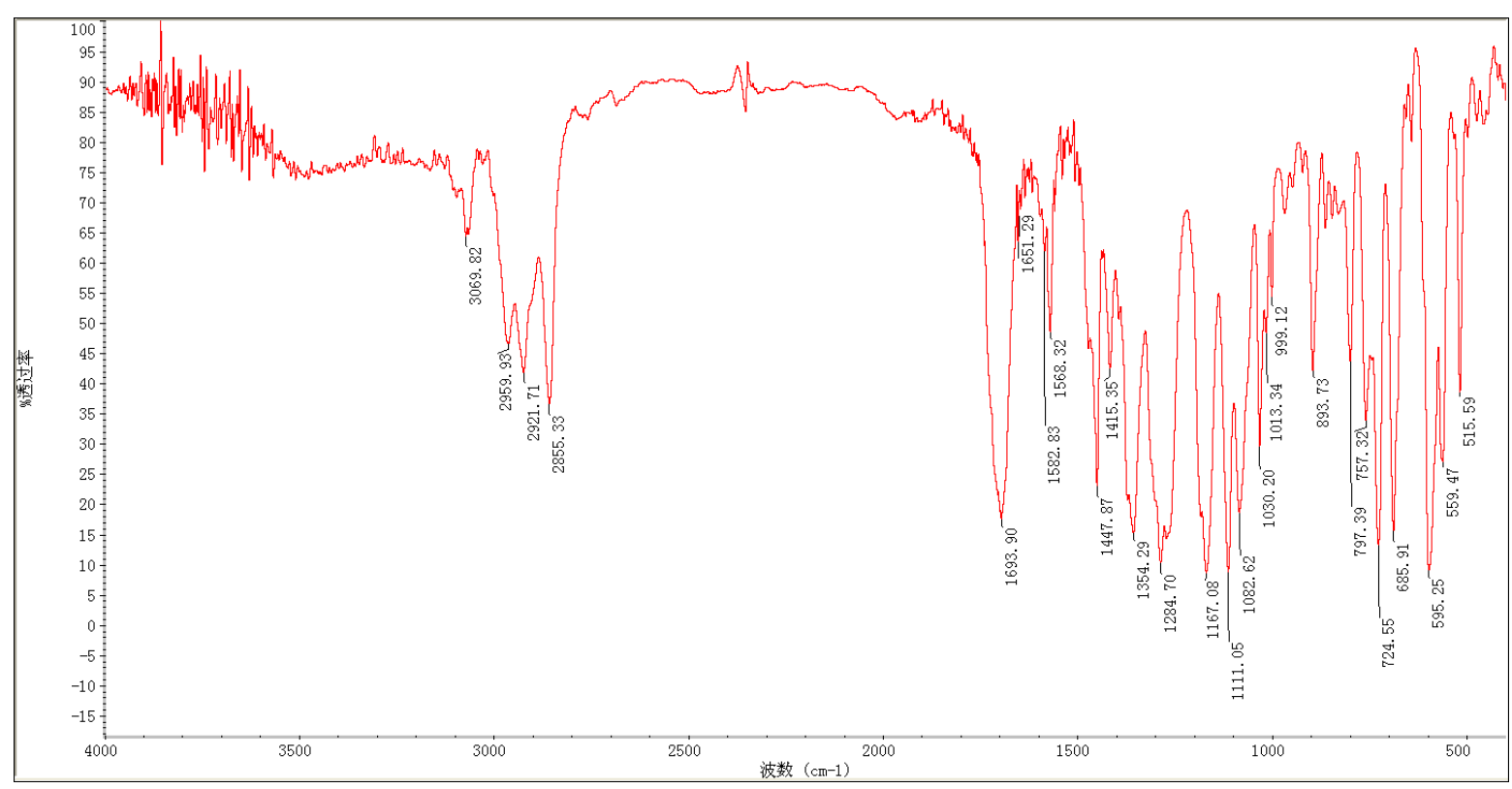

IR Spectrum of Compound 5q

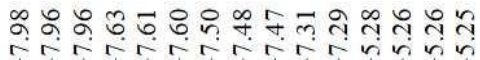

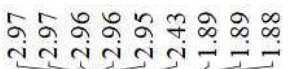<smiles></smiles>

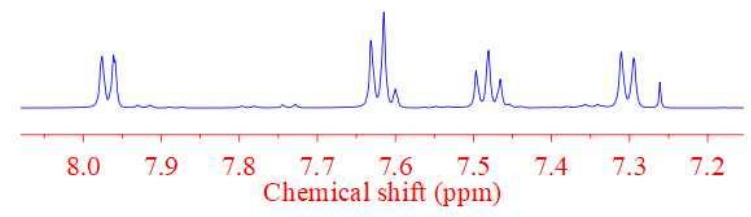

${ }^{1} \mathrm{H} \mathrm{NMR}\left(\mathrm{CDCl}_{3}, 500 \mathrm{MHz}\right)$

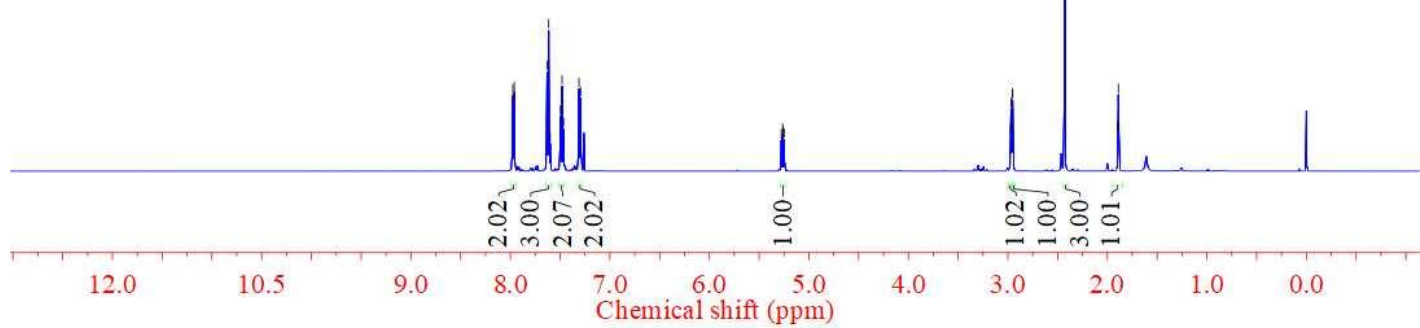

${ }^{1} \mathrm{H}$ NMR Spectrum of Compound 6a 


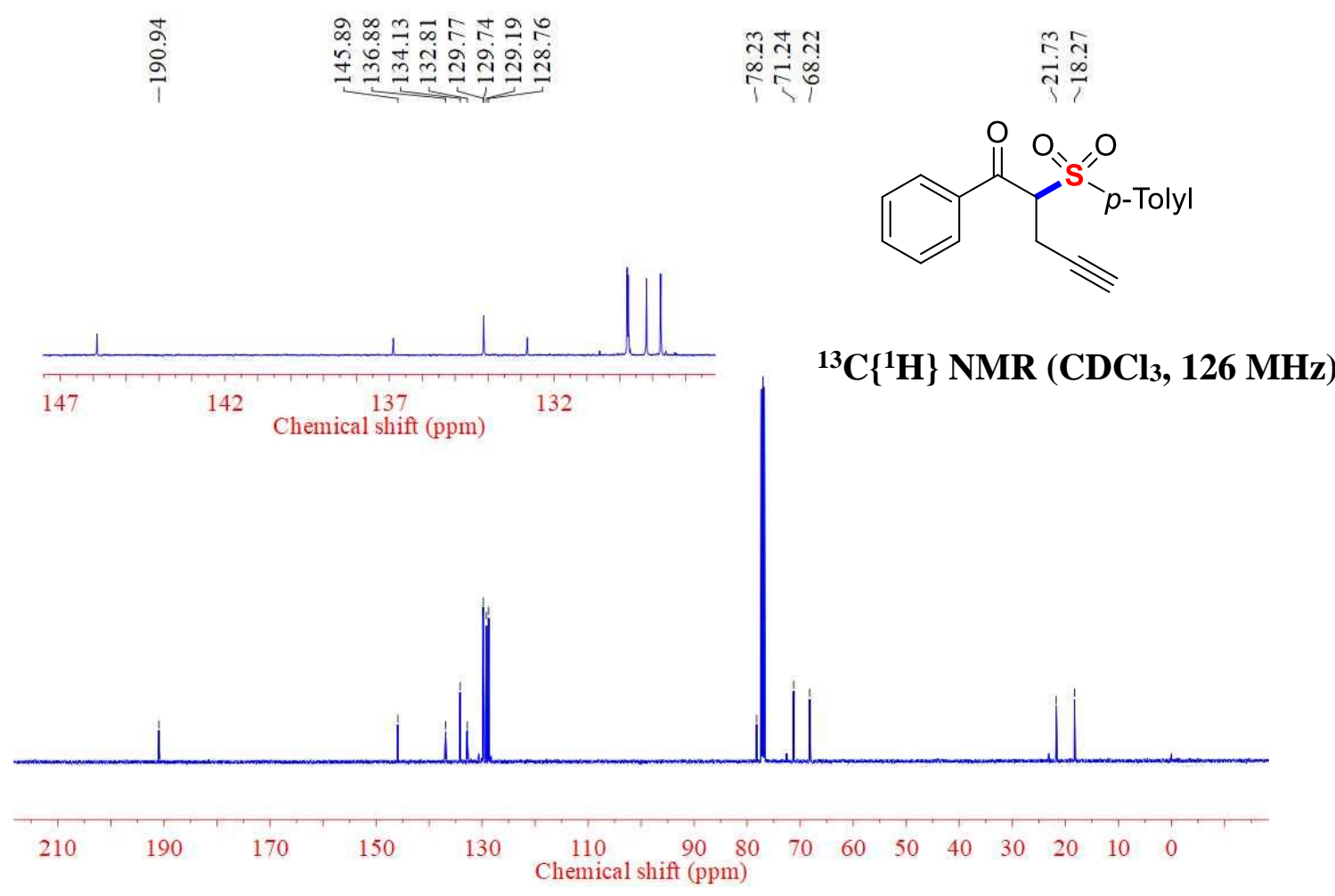

${ }^{13} \mathbf{C}\left\{{ }^{1} \mathrm{H}\right\}$ NMR Spectrum of Compound 6a

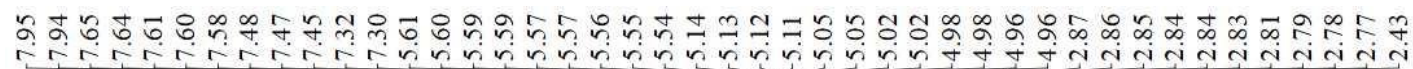

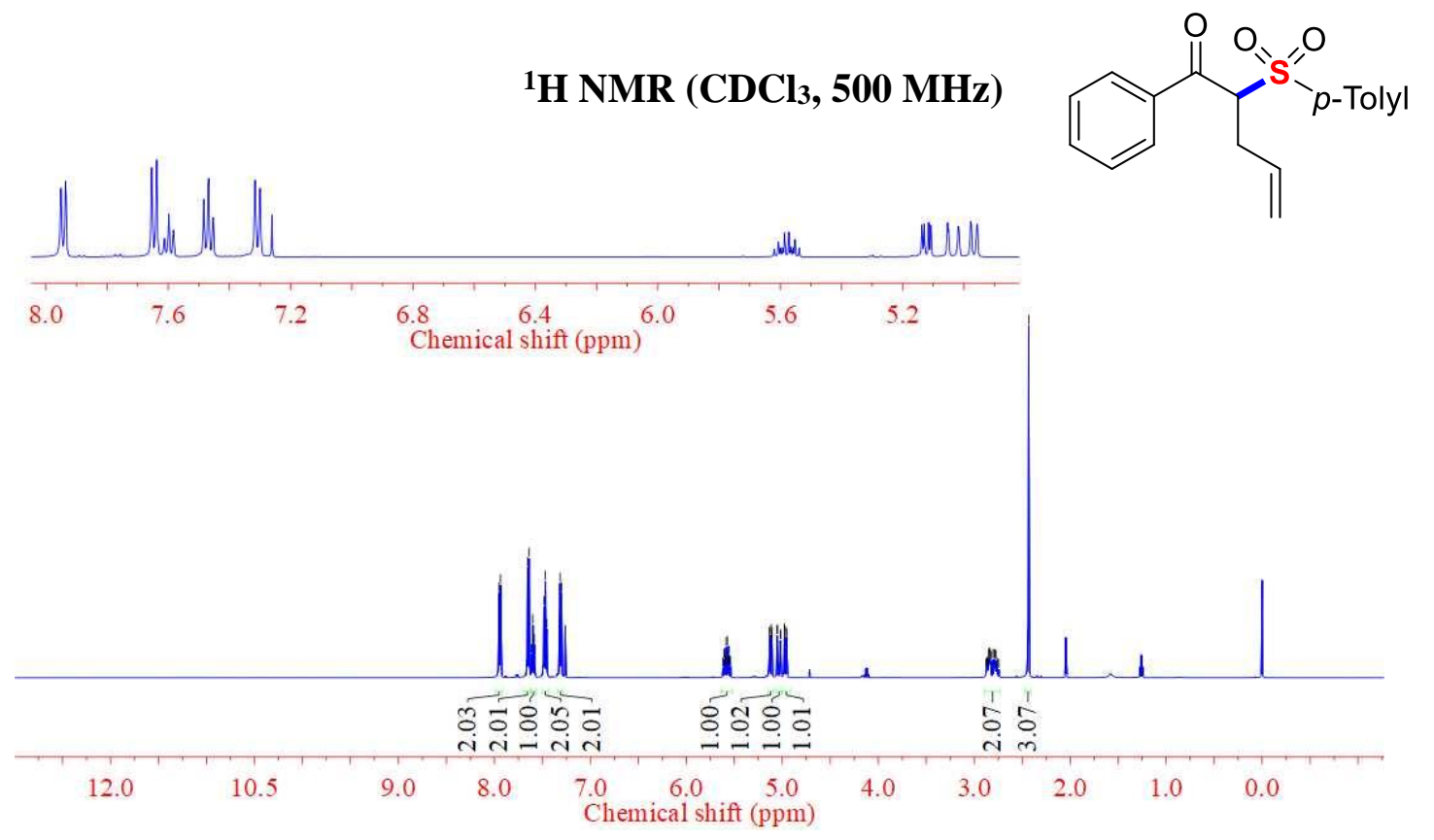

${ }^{1} \mathrm{H}$ NMR Spectrum of Compound $6 \mathrm{~b}$ 


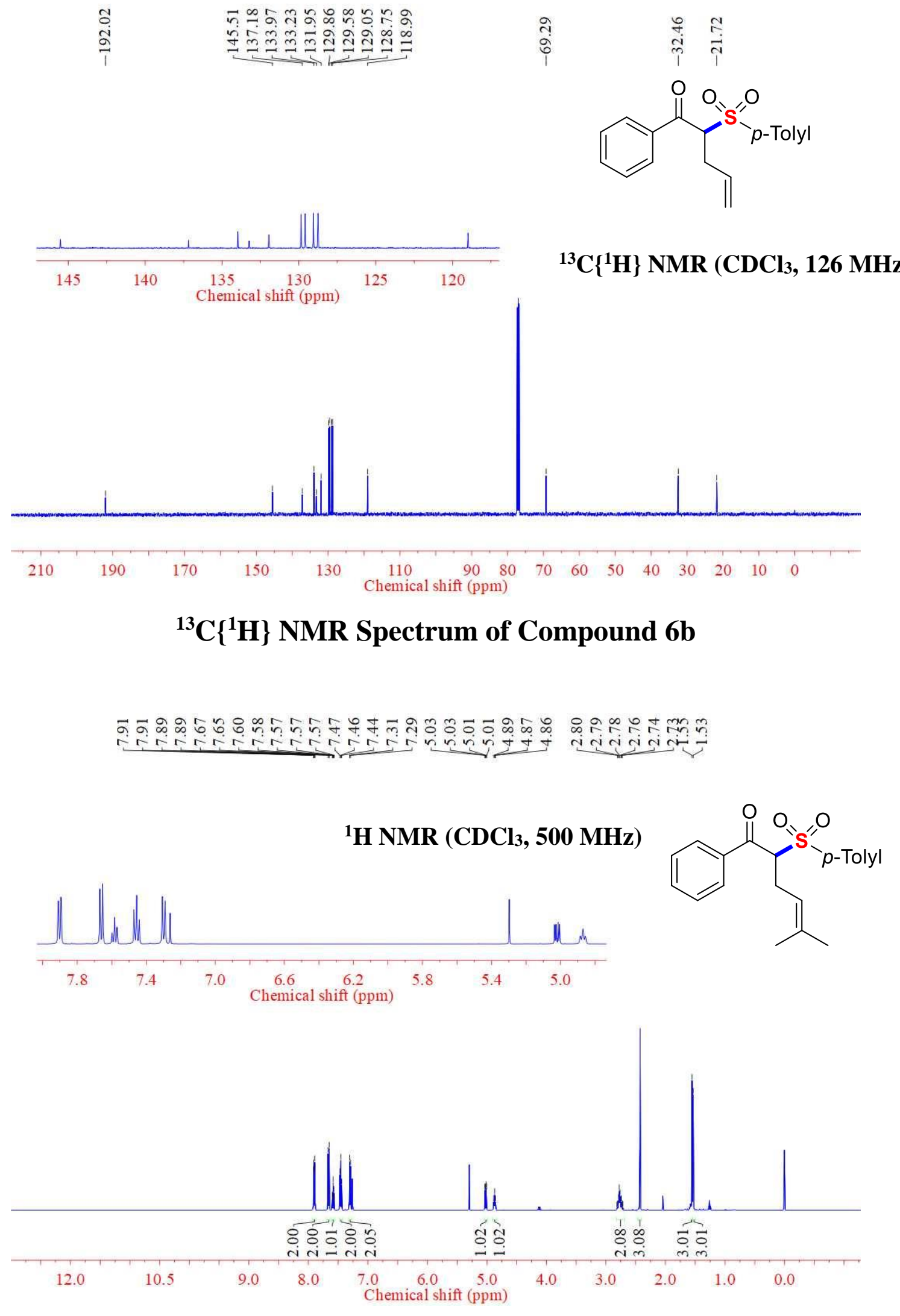

${ }^{1} \mathrm{H}$ NMR Spectrum of Compound $6 \mathrm{c}$ 


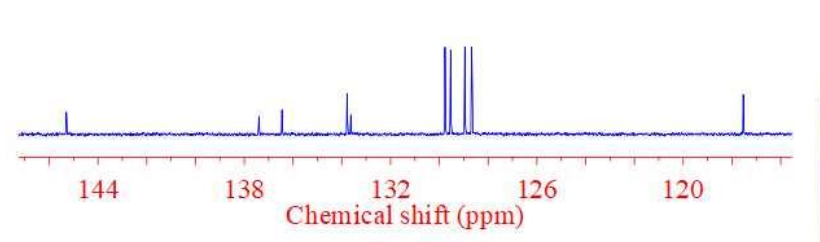<smiles>CC(C)=CCC(C(=O)c1ccccc1)S(=O)(=O)O[Na]</smiles>

${ }^{13} \mathrm{C}\left\{{ }^{1} \mathrm{H}\right\}$ NMR $\left(\mathrm{CDCl}_{3}, 126 \mathrm{MHz}\right)$

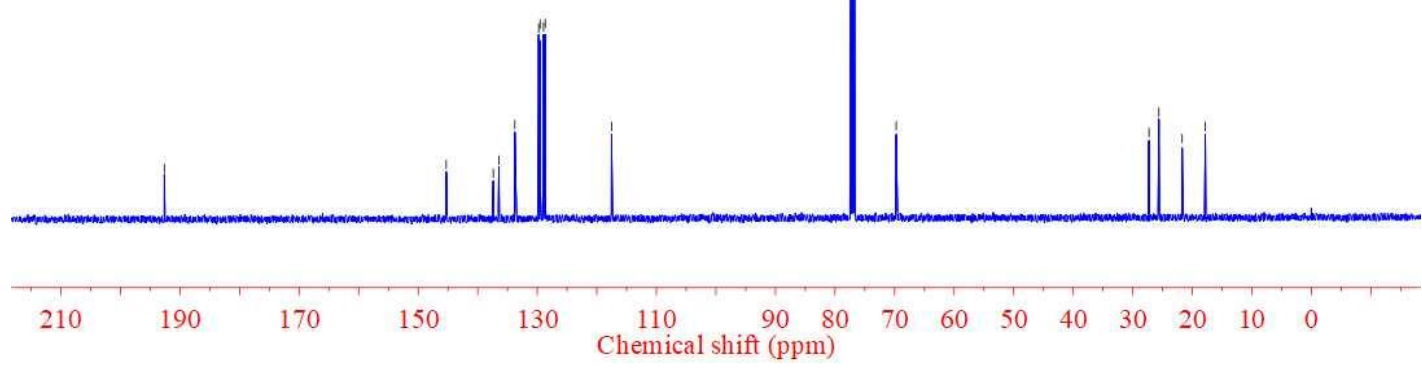

${ }^{13} \mathbf{C}\left\{{ }^{1} \mathrm{H}\right\}$ NMR Spectrum of Compound $6 \mathrm{c}$

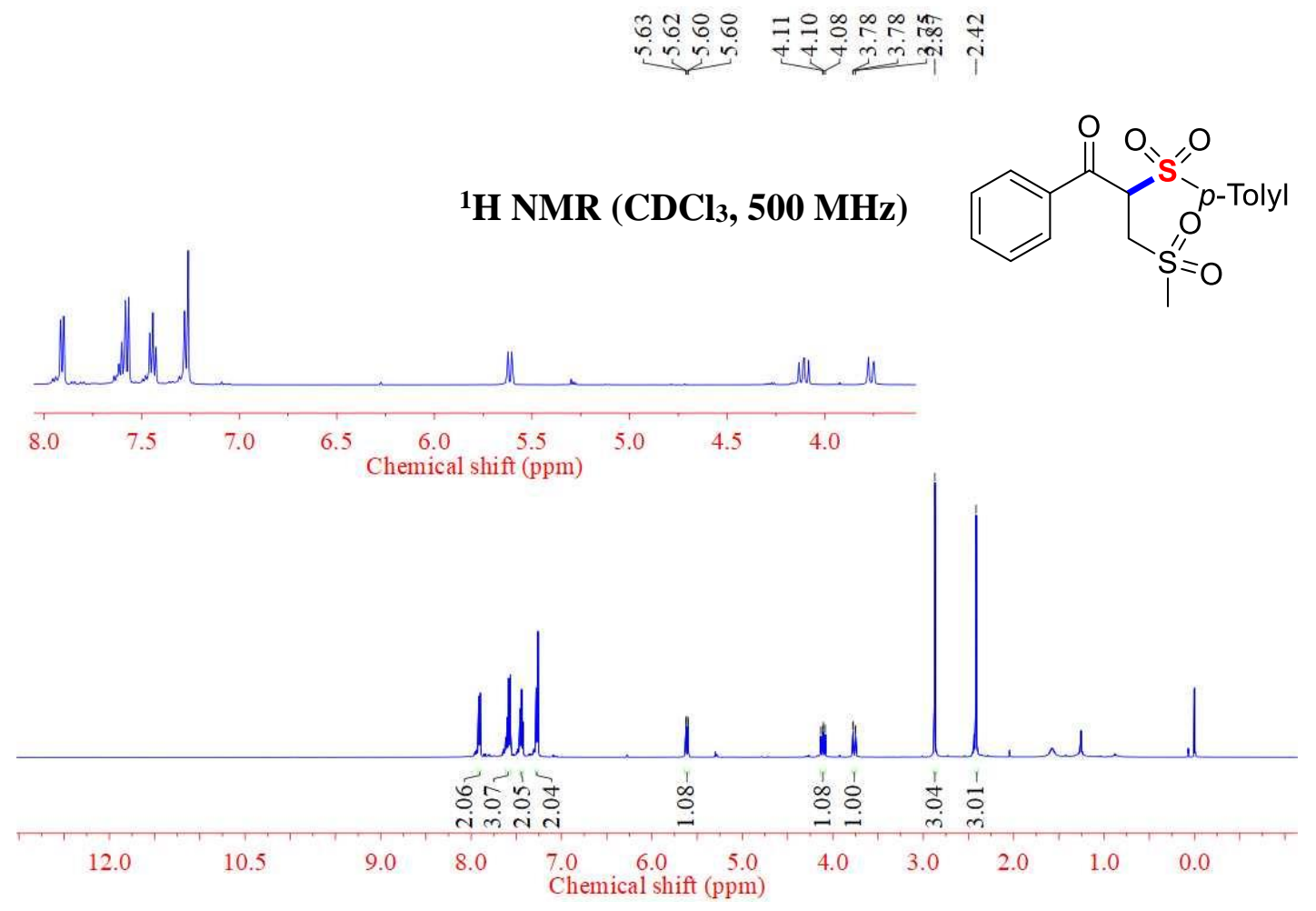

${ }^{1} \mathrm{H}$ NMR Spectrum of Compound $6 \mathrm{~d}$ 


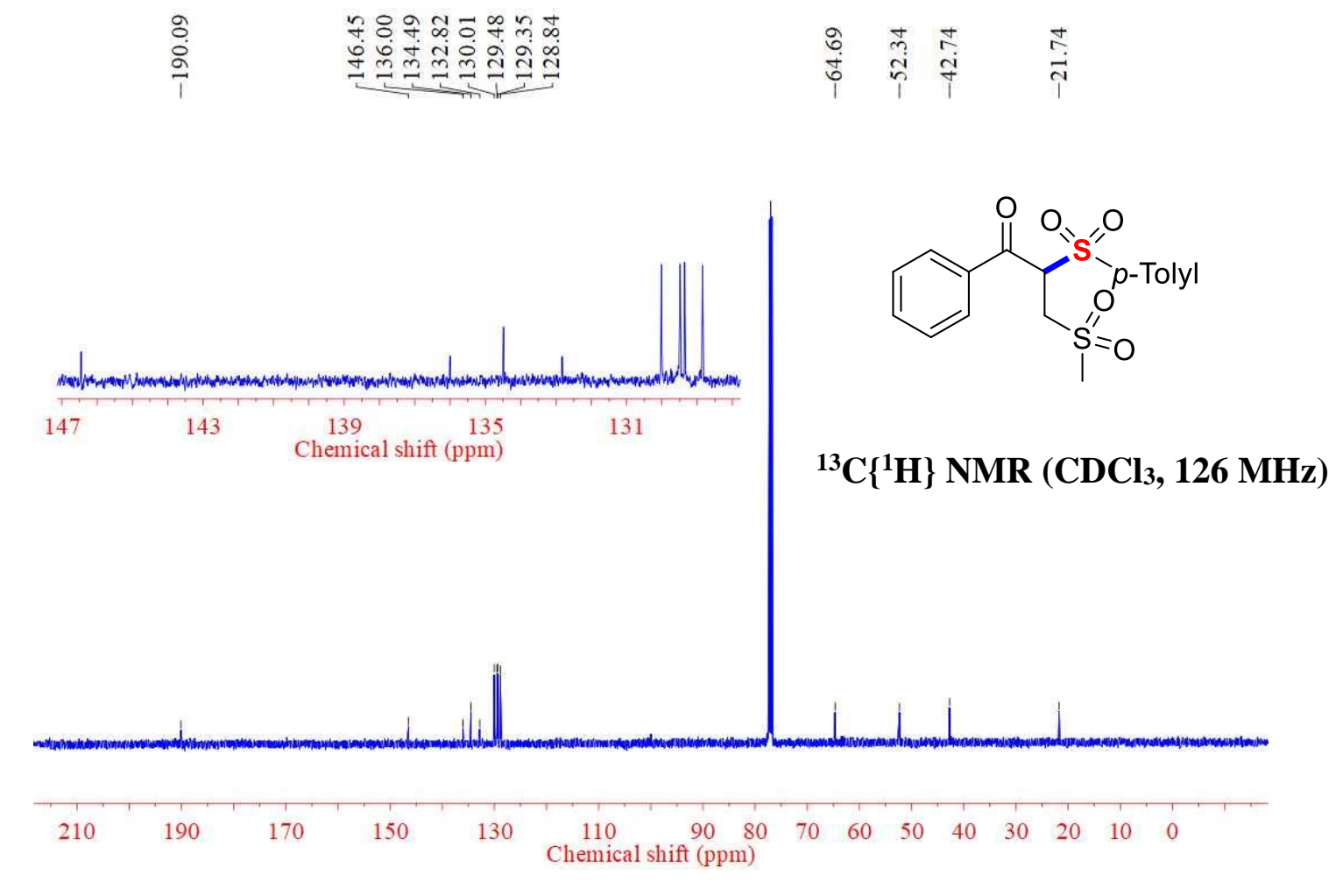

\section{${ }^{13} \mathrm{C}\left\{{ }^{1} \mathrm{H}\right\}$ NMR Spectrum of Compound $6 \mathrm{~d}$}

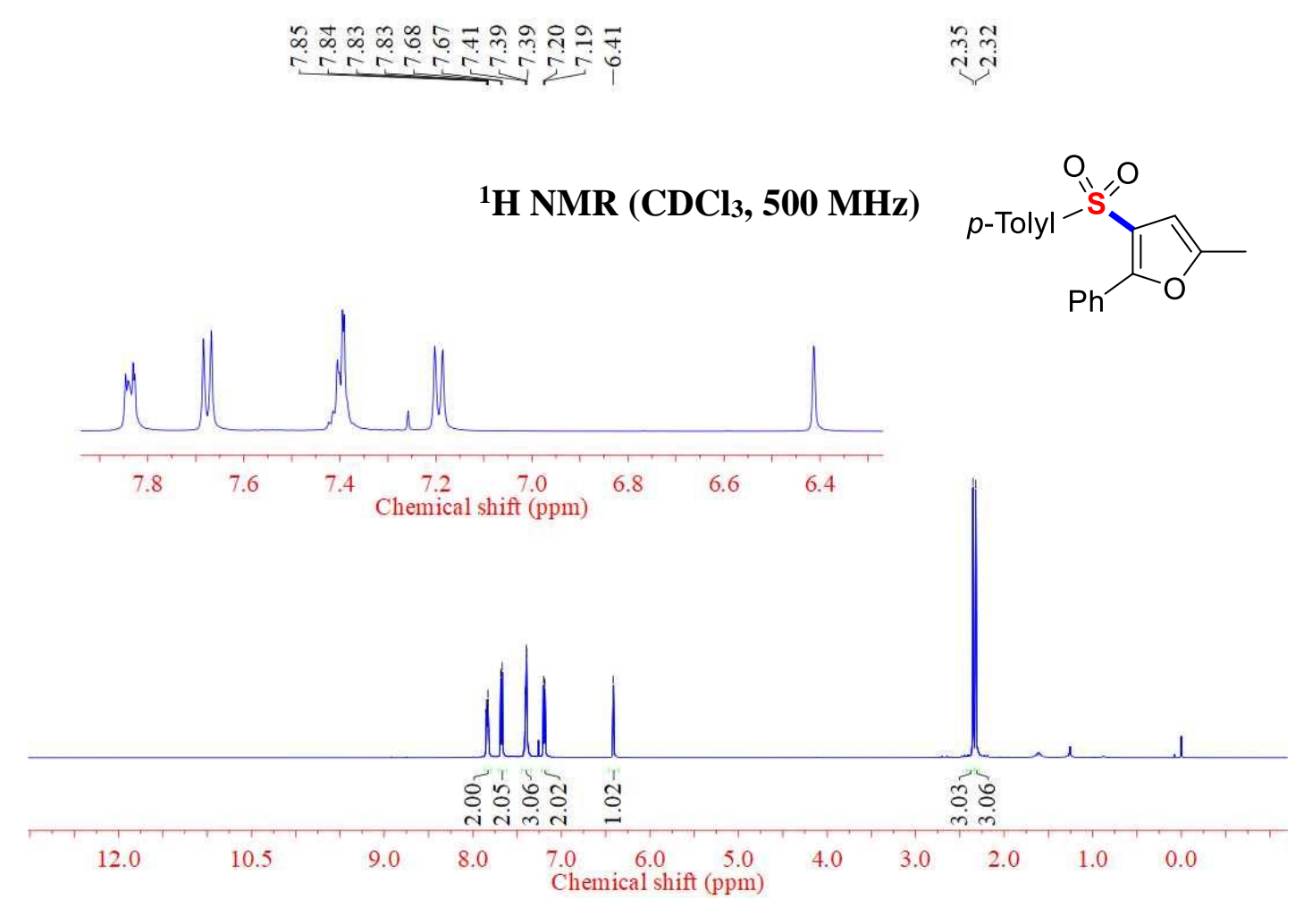

${ }^{1}$ H NMR Spectrum of Compound 7a 


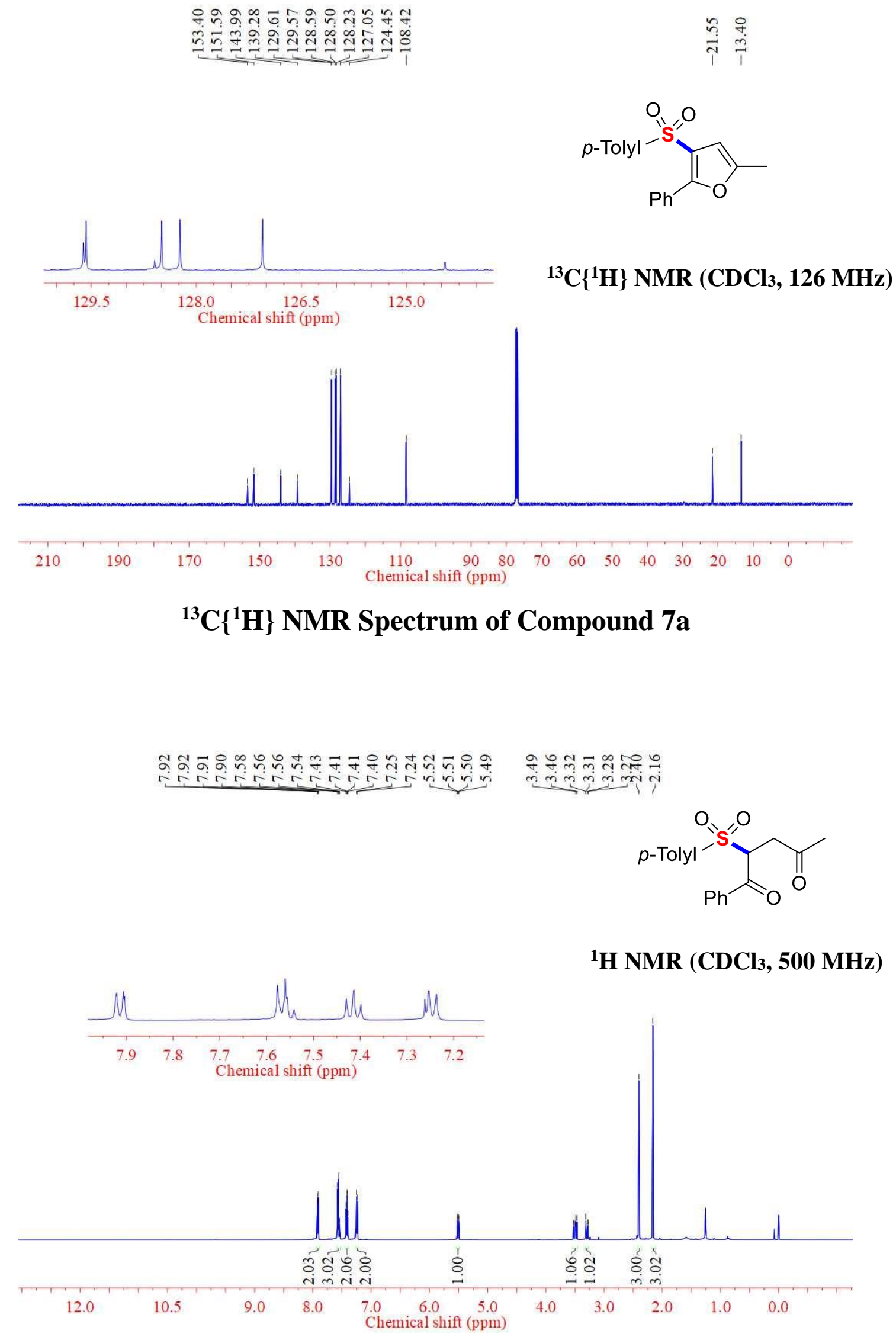

${ }^{1} \mathrm{H}$ NMR Spectrum of Compound 7b 


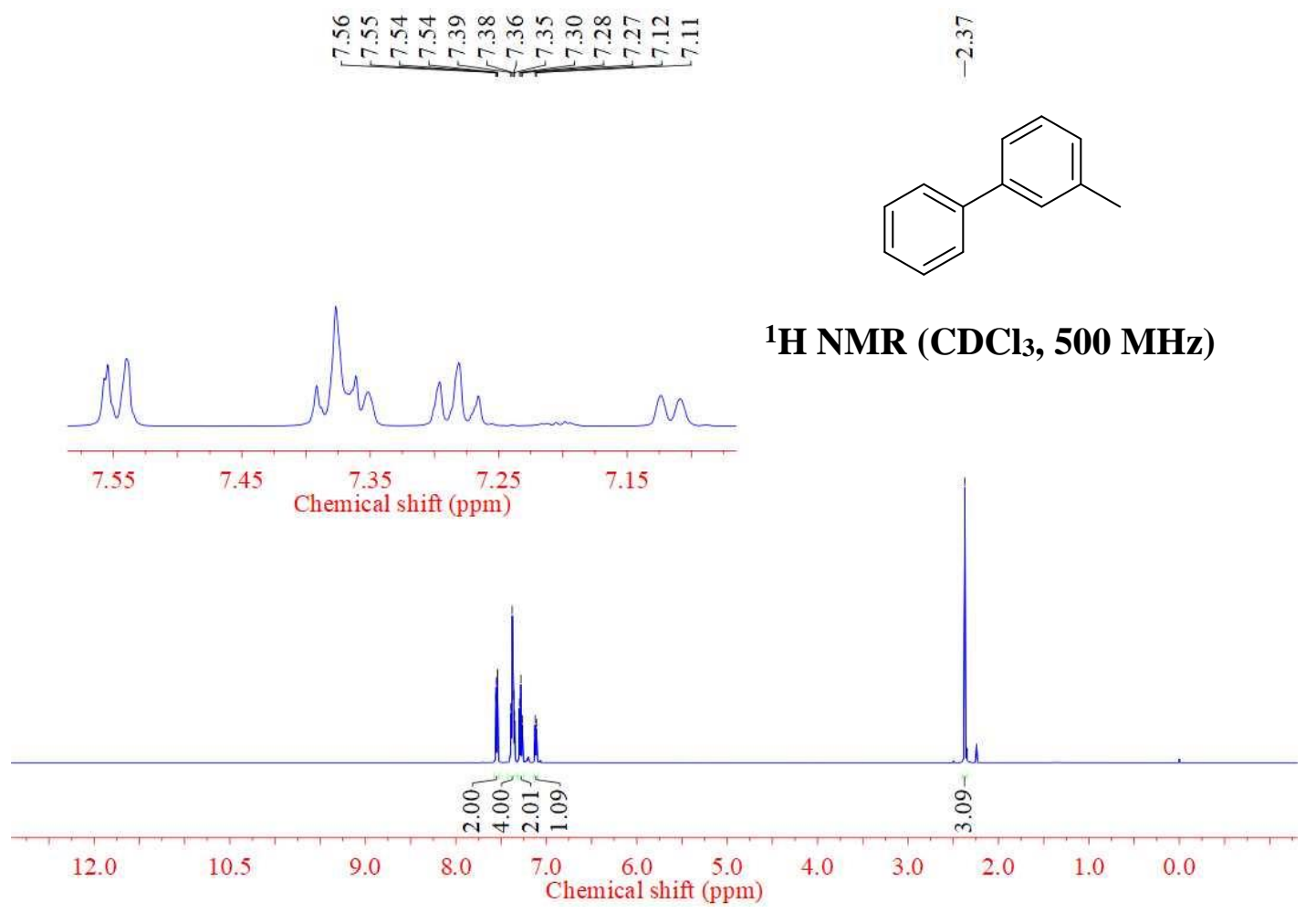

${ }^{1} \mathrm{H}$ NMR Spectrum of Compound 7c

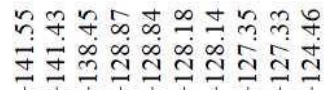

${ }^{13} \mathrm{C}\left\{{ }^{1} \mathrm{H}\right\} \mathrm{NMR}\left(\mathrm{CDCl}_{3}, 126 \mathrm{MHz}\right)$
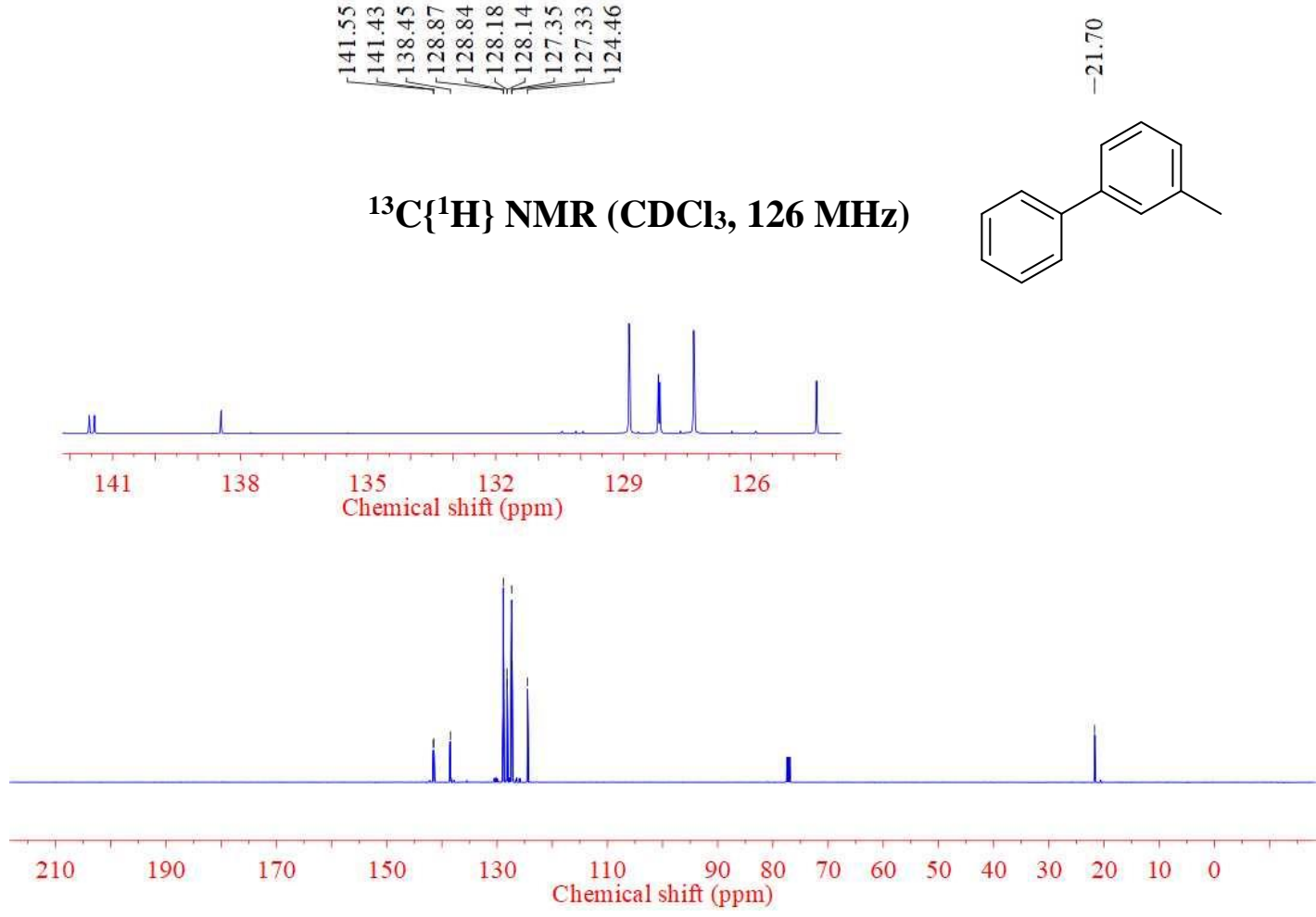

${ }^{13} \mathrm{C}\left\{{ }^{1} \mathrm{H}\right\}$ NMR Spectrum of Compound 7c 
<smiles>Cc1nc2ccccc2nc1-c1ccccc1</smiles>

${ }^{1} \mathrm{H}$ NMR (CDCl3, $\left.500 \mathrm{MHz}\right)$
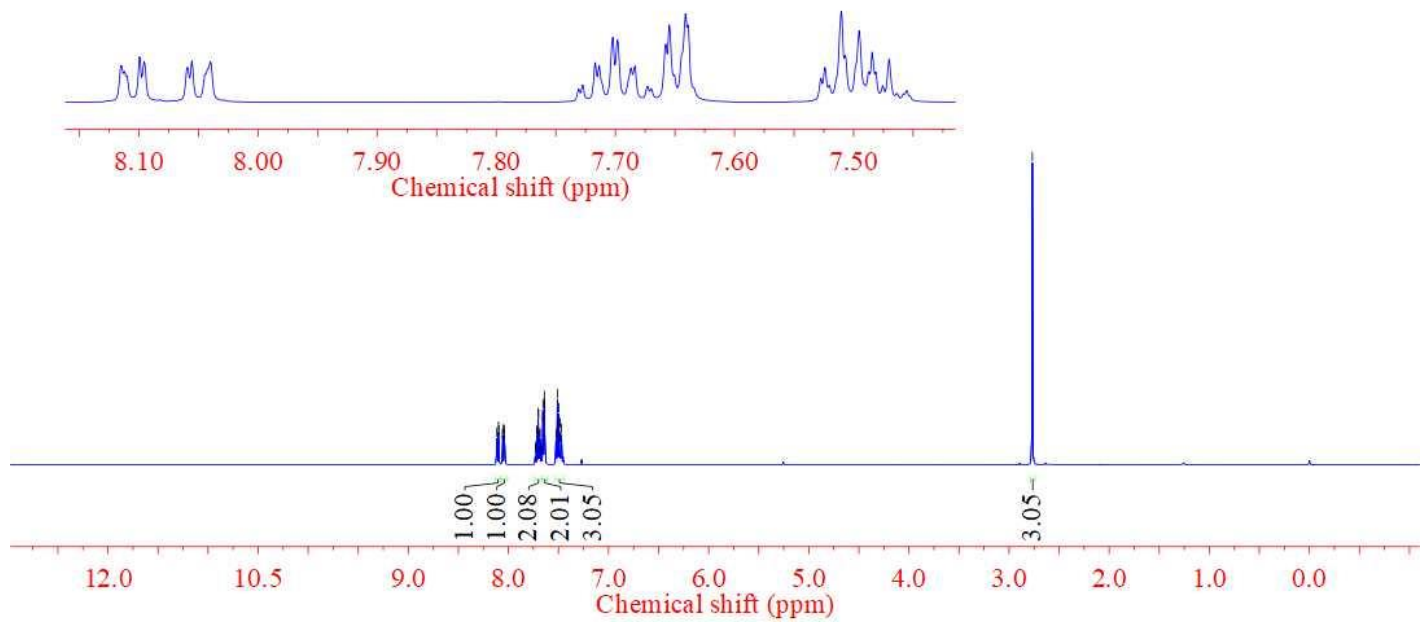

${ }^{1}$ H NMR Spectrum of Compound 7d

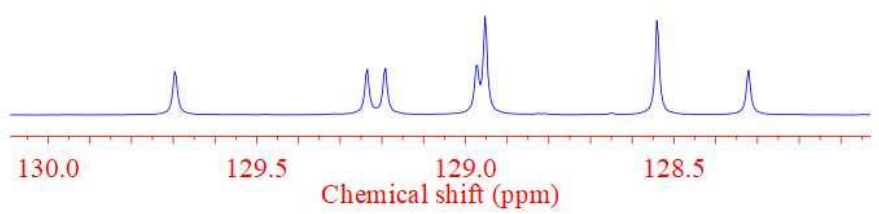<smiles>Cc1nc2ccccc2nc1-c1ccccc1</smiles>

${ }^{13} \mathrm{C}\left\{{ }^{1} \mathrm{H}\right\} \mathrm{NMR}(\mathrm{CDCl}, 126 \mathrm{MHz})$

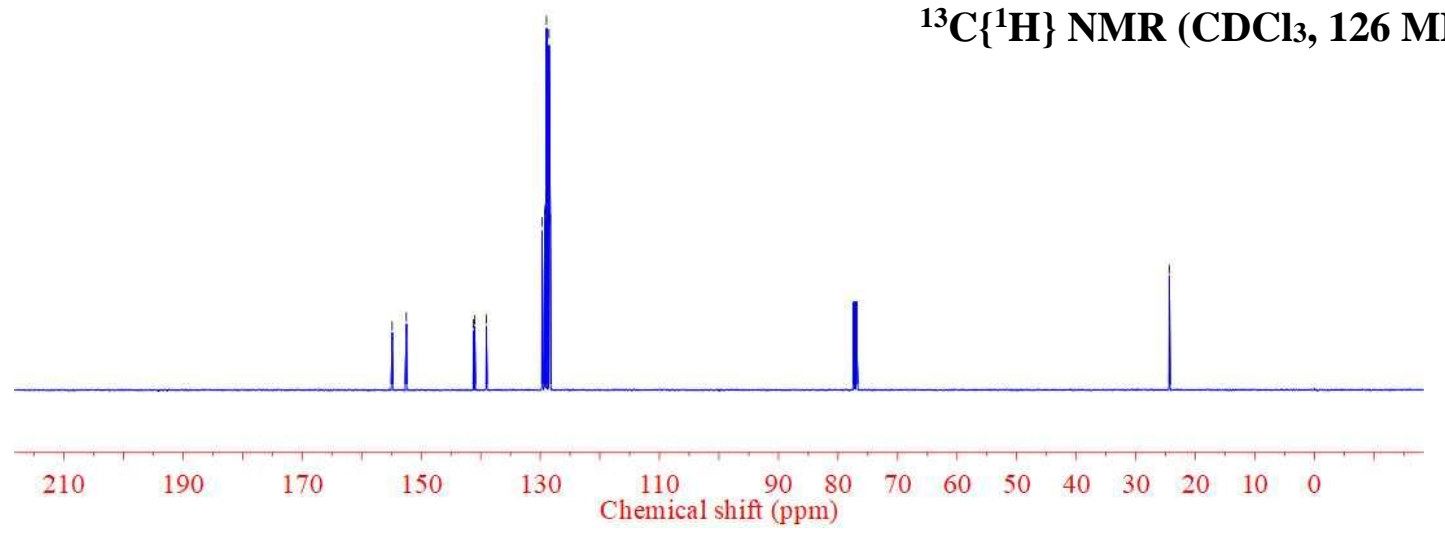

${ }^{13} \mathbf{C}\left\{{ }^{1} \mathbf{H}\right\}$ NMR Spectrum of Compound 7d 
<smiles>O=S(C=C(c1ccccc1)c1ccccc1)c1ccccc1</smiles>

${ }^{1} \mathrm{H}$ NMR $\left(\mathrm{CDCl}_{3}, 500 \mathrm{MHz}\right)$
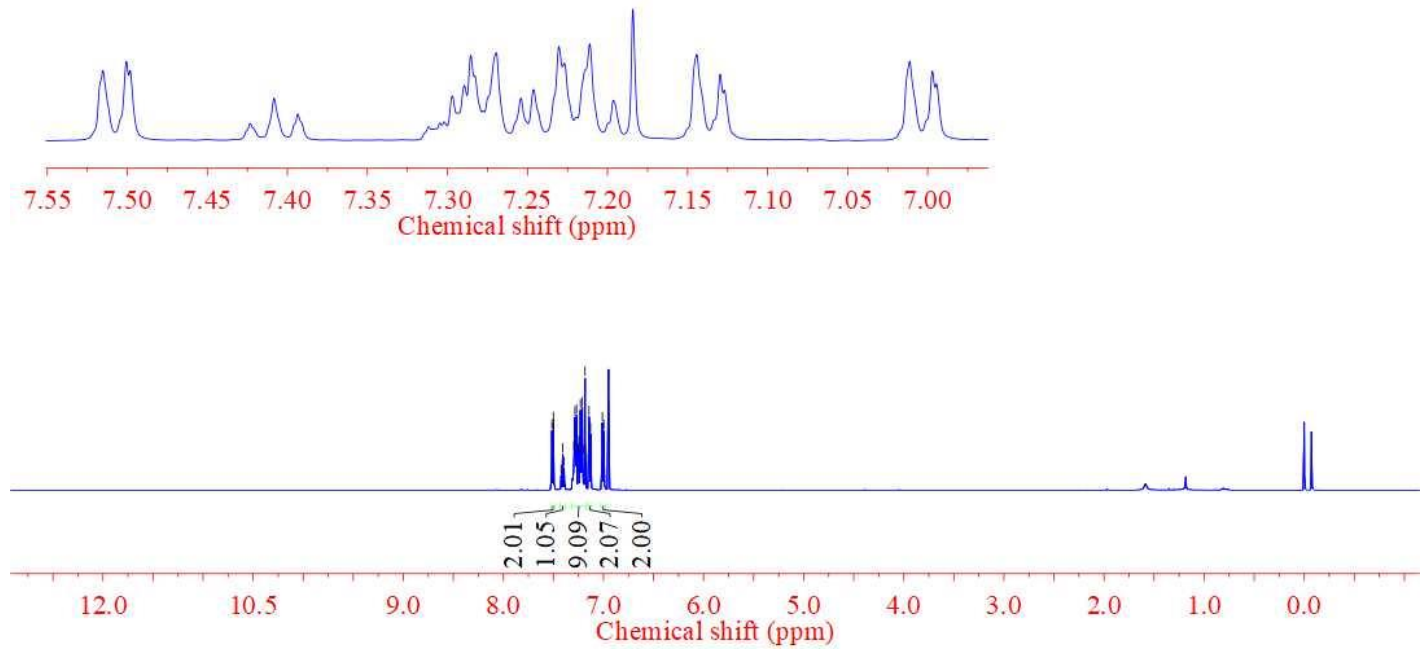

${ }^{1}$ H NMR Spectrum of Compound 8

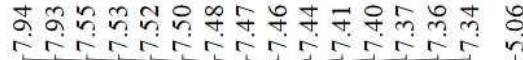<smiles>N/C(=C\[SH](=O)(O)c1ccccc1)c1ccccc1</smiles>

${ }^{1} \mathrm{H}$ NMR $\left(\mathrm{CDCl}_{3}, 500 \mathrm{MHz}\right)$

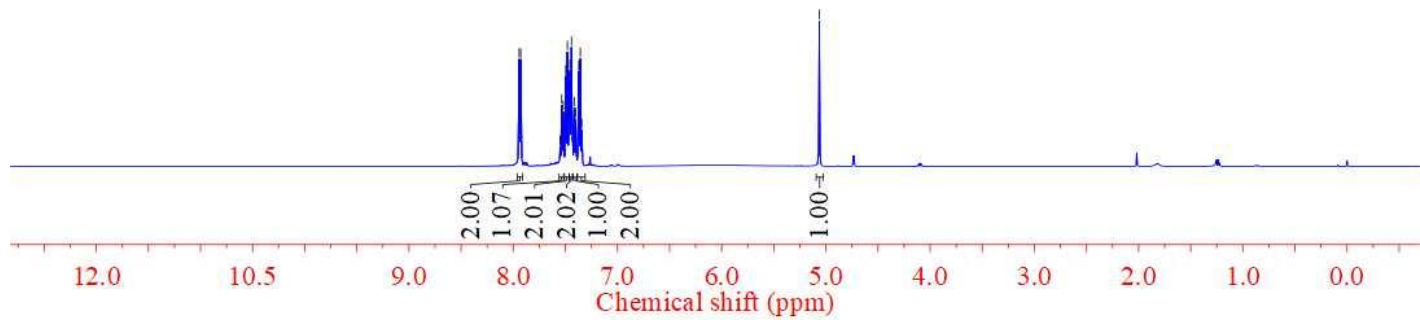

${ }^{1} \mathrm{H}$ NMR Spectrum of Compound E 
<smiles>N/C(=C/SO[R6](=O)c1ccccc1)c1ccccc1</smiles>

${ }^{13} \mathrm{C}\left\{{ }^{1} \mathrm{H}\right\} \mathrm{NMR}(\mathrm{CDCl}, 126 \mathrm{MHz})$

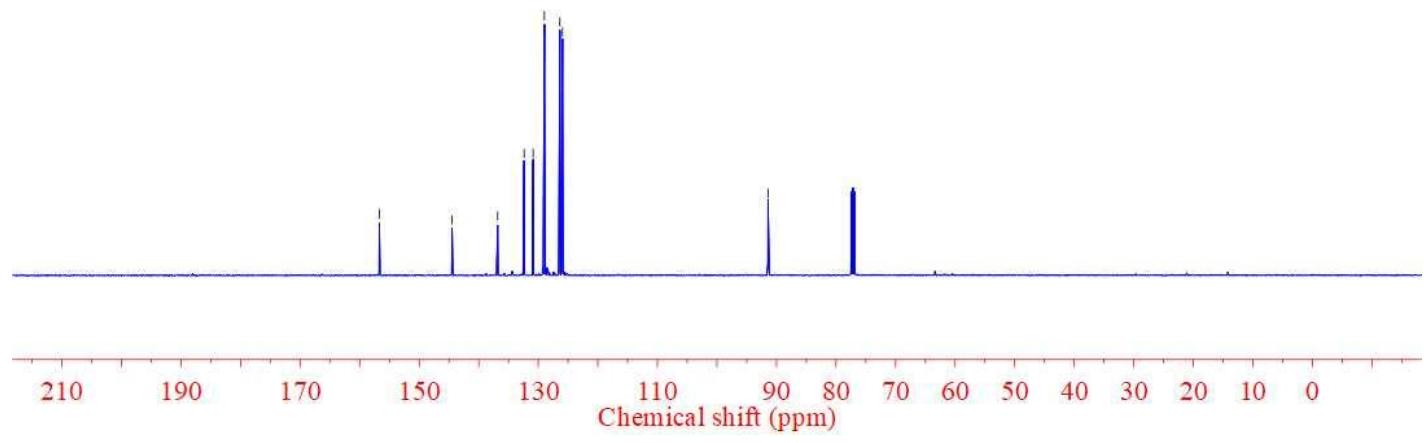

\section{${ }^{13}$ C NMR Spectrum of Compound E}

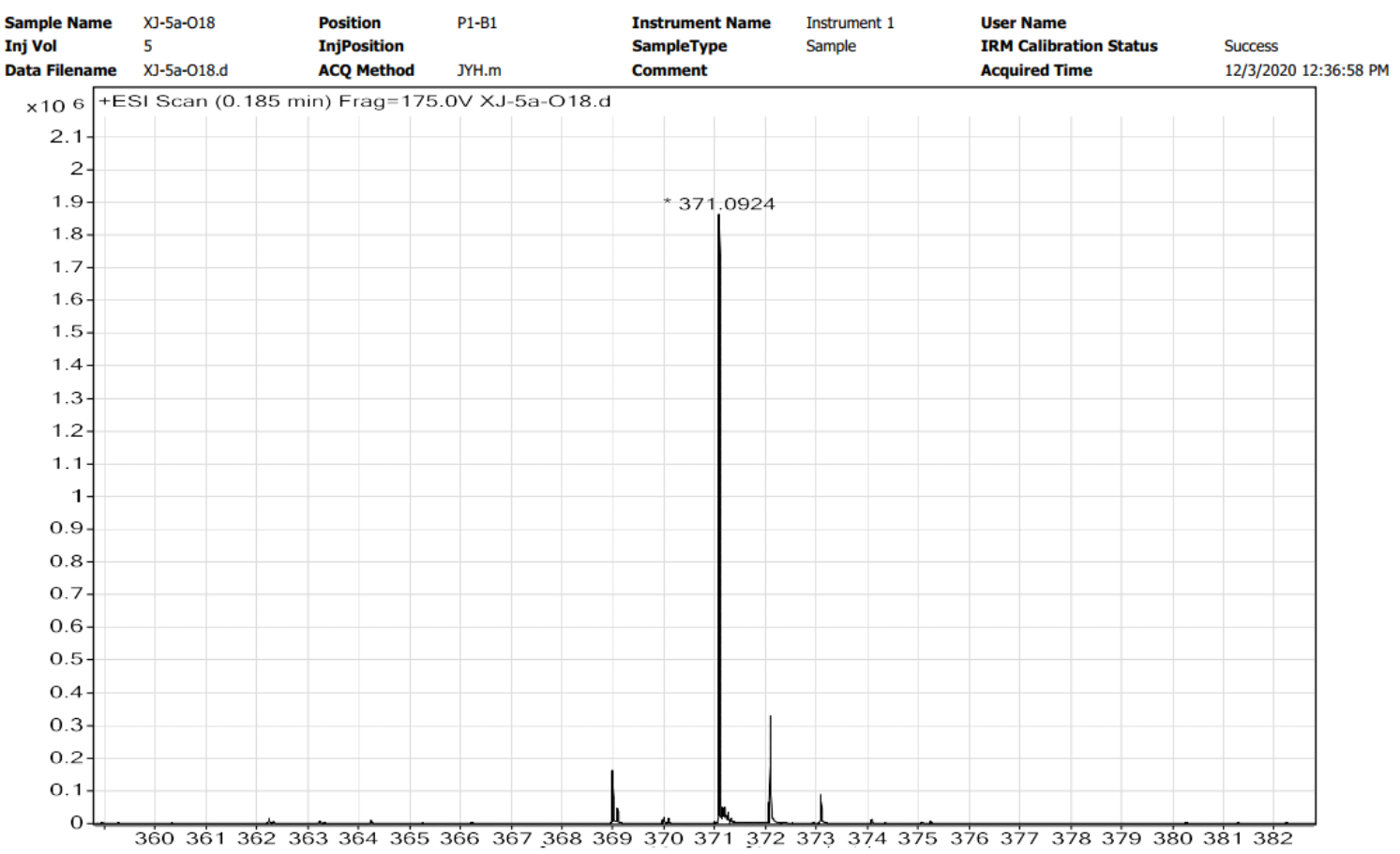

\begin{tabular}{|c|c|c|c|c|c|c|c|}
\hline Formula (M) & Score (MFG) & Mass & Mass (MFG) & $\mathrm{m} / \mathrm{z}$ (Calc) & Diff (ppm) & DBE & $\mathrm{m} / \mathrm{z}$ \\
\hline $\mathrm{C}_{17} \mathrm{H}_{18} \mathrm{~N}_{2} \mathrm{O}_{3}\left[{ }^{18} \mathrm{O}\right] \mathrm{S}$ & 99.87 & 348.1032 & 348.103 & 371.0922 & -0.59 & 10 & 371.0924 \\
\hline
\end{tabular}

